\title{
Physical activity and lifestyle behaviours in obesity and the prevention of type 2 diabetes: The PREVIEW Project
}

\author{
Swindell, Nils Joseph
}

How to cite:

Swindell, Nils Joseph (2018) Physical activity and lifestyle behaviours in obesity and the prevention of type 2 diabetes: The PREVIEW Project. Doctoral thesis, Swansea University.

http://cronfa.swan.ac.uk/Record/cronfa43702

Use policy:

This item is brought to you by Swansea University. Any person downloading material is agreeing to abide by the terms of the repository licence: copies of full text items may be used or reproduced in any format or medium, without prior permission for personal research or study, educational or non-commercial purposes only. The copyright for any work remains with the original author unless otherwise specified. The full-text must not be sold in any format or medium without the formal permission of the copyright holder. Permission for multiple reproductions should be obtained from the original author.

Authors are personally responsible for adhering to copyright and publisher restrictions when uploading content to the repository.

Please link to the metadata record in the Swansea University repository, Cronfa (link given in the citation reference above.)

http://www.swansea.ac.uk/library/researchsupport/ris-support/ 


\section{(19ix \\ .

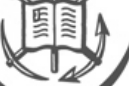 \\ Swansea University \\ Prifysgol Abertawe}

Physical activity and lifestyle behaviours in obesity and the prevention of type 2 diabetes:

The PREVIEW Project

Nils Joseph Swindell

Submitted to Swansea University in fulfilment of the requirements for the Degree of Doctor of Philosophy

Swansea University

2018 


\subsection{Abstract}

Interventions are needed to stem the rise of obesity and type 2 diabetes. Physical activity (PA) is integral to lifestyle interventions, however, a paucity of research applying objective measures of PA exists in populations at risk of diabetes. Insight into changing PA and dietary behaviours is required to develop effective interventions. The aim of this thesis was to capture an insight into high-risk behaviours and behaviour change in pre-diabetic adults, and assess the feasibility of running an intervention in overweight and obese children.

Study 1 investigated the associations between objectively measured PA and cardio-metabolic risk factors in pre-diabetic adults from 8 countries. Results indicated that moderate-to-vigorous physical activity (MVPA) was negatively associated with cardio-metabolic risk factors. However, associations between total PA and all risk factors were at least as strong as than MVPA.

Study 2 examined the psychosocial correlates of objectively measured PA. Results showed that habit-strength and exercise intentions were negatively and positively associated with MVPA respectively. Two-way interactions between peer support and inactivity temptations and between age and social support, suggested that social support was of greater importance in older participants and in the presence of inactivity temptations. Associations between PA self-efficacy and goal adjustment were country specific.

Study 3 investigated associations between body fat $\%$ and lifestyle behaviours in 15,977 children aged 9-11yrs. Multilevel-models revealed body fat\% was negatively associated with active transport to school, full fat milk and sweetened beverage consumption. Later bed time was positively associated with body fat $\%$. No change was found in associations over a 10 -year period.

Study 4 used mixed methods case studies combining participant perceptions of the behaviour change process with objective outcome measures to assess the feasibility of running an intervention in children. Three of the four cases showed improvements in dietary and PA behaviour and reductions in BMI z-score, HOMA-IR and HbA1c. Semi-structured interviews indicated that behavioural changes occurred despite not always being detected by objective measures, possibly due to compensation effects or seasonal changes. Furthermore, goal setting was considered useful but planning goals waned throughout the study. Compliance with self-monitoring techniques was low and largely reliant on parents. 


\subsection{Summary of publications}

Swindell N, Mackintosh K.A, McNarry M.A, Stephens J.W, Sluik D, Fogelholm M, Drummen M, MacDonald I, Martinez J.A, Handjieva-Darlenska T, Poppitt S.D, Brand-Miller J, Larsen T, Raben A and Stratton G. Objectively Measured Physical Activity and Sedentary Time Are Associated With Cardiometabolic Risk Factors in Adults With Prediabetes: The PREVIEW Study. Diabetes Care 41, dc171057 (2017)

Swindell N, Hansen S, Mackintosh K.A, McNarry M.A, Stephens J.W, Sluik D, Navas-Carreto S, Fogelholm M, Drummen M, MacDonald I, Martinez J.A, Handjieva-Darlenska T, Poppitt S.D, Brand-Miller J, Larsen T, Raben A, Schlicht $\mathrm{W}$ and Stratton G. Personal and psychosocial correlates of physical activity in adults with pre-diabetes from 8 countries: the PREVIEW study. Under review International Journal of Behavioural Medicine

Swindell N, Berridge D, McNarry M.A, Mackintosh K.A, Boddy L.M, Fairclough S.J and Stratton G. Lifestyle behaviours associated with body fat percent in 9-11-year-old children. Under review International Journal of Obesity 


\subsection{Declarations and Statements}

1. I, Nils Joseph Swindell, hereby declare that the work presented in this thesis has not previously been accepted in substance for any degree and is not being concurrently submitted in candidature for any degree.

2. I, Nils Joseph Swindell, hereby declare that the thesis is the result of my own investigations, except where otherwise stated and that other sources are acknowledged by footnotes giving explicit references and that a bibliography is appended. In chapters 5 and 6, data from the PREVIEW project was analysed. In these chapters, I was responsible for the analysis of accelerometer data, statistical analysis and the presentation of the manuscript. I was not involved in recruitment or data collection. In chapter 7 data was analysed from the SportsLinx study, I was responsible for statistical analysis, and preparation of the manuscript but was not involved in data collection. In chapter 8, I was responsible for recruitment, delivery of the intervention, data collection, analysis and presentation of manuscript.

3. I, Nils Joseph Swindell, hereby gives consent for the thesis, if accepted, to be available for photocopying and for inter-library loan, and for the title and summary to be made available to outside organisations.

Signed: Scylatsello (candidate)

Date: $28 / 02 / 2018$ 


\subsection{Contents Page}

1.1 Abstract 2

$\begin{array}{llr}1.2 & \text { Summary of publications } & 3\end{array}$

1.3 Declarations and Statements 4

1.4 Contents Page $\quad 5$

1.5 Acknowledgments $\quad 8$

1.6 List of Tables and Figures 9

$\begin{array}{ll}1.7 \text { List of abbreviations } & 10\end{array}$

$2 \quad$ Introduction $\quad 13$

2.1 Problem statement 14

2.2ßThesis aims $\quad 15$

3 Literature review $\quad 16$

3.1 Introduction $\quad 16$

3.2 Pathophysiology of type 2 diabetes 16

$\begin{array}{ll}3.3 \text { Prevalence } & 18\end{array}$

3.3.1 Adults 18

3.3.2 Children 18

3.4 Impact of type 2 diabetes $\quad 20$

3.4.1 Mortality 20

3.4.2 Morbidity 21

3.4.3 Financial 21

3.5 Risk Factors $\quad 22$

3.5.1 Non-modifiable risk factors 22

3.5.2 Modifiable risk factors 24

3.5.3 Diet 24

3.5.4 Physical activity $\quad 27$

3.5.5 Physical activity intensity 28

3.5.6 Sedentary time 29

3.5.7 Metabolic syndrome 29

3.6 Correlates of physical activity $\quad 31$

3.7 Behaviour change $\quad 34$

3.8 Lifestyle interventions $\quad 39$

3.8.1 Adults 39

3.8.2 Physical activity in lifestyle interventions 42

3.8.3 Physical activity intensity 43

3.8.4 Children 44

3.8.5 Lifestyle behaviours associated with obese children 45

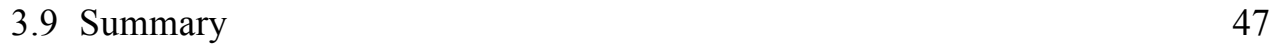

3.10Objectives $\quad 49$

4 Methods $\quad \mathbf{5 0}$

4.1 Study 1 and 2 PREVIEW intervention $\quad 50$

4.1.1 Participants and settings 50

4.1.2 Instruments and Procedures 51

4.1.3 Design 57 
4.2 Study 4: The effectiveness of a child weight management intervention; a mixed methods investigation of child and parent's perceptions of the behaviour change process $\quad 57$

4.2.1 Participants/settings 58

4.2.2 Instruments and Procedures 58

4.2.3 Design 64

4.3 Study 3: Lifestyle behaviours associated with body fat percent in 9-11-yearold children: The SportsLinx study 65

4.3.1 Participants and Settings 65

4.3.2 Instruments and Procedures $\quad 65$

4.1 Thesis Map 68

5 Study 1: Objectively Measured Physical Activity and Sedentary Time are Associated with Cardio-metabolic Risk Factors in Adults with Prediabetes: The PREVIEW Study ${ }^{314}$

5.1 Introduction

$\begin{array}{ll}5.2 \text { Methods } & 70\end{array}$

$\begin{array}{lll}\text { 5.2.1 Participants and settings } & 70\end{array}$

$\begin{array}{lll}5.2 .2 & \text { Statistical analysis } & 71\end{array}$

5.2.3 Results 73

$\begin{array}{ll}5.3 \text { Discussion } & 79\end{array}$

5.4 Conclusion $\quad 84$

5.5 Thesis Map $\quad 85$

6 Study 2: Personal and psychosocial correlates of physical activity in adults with pre-diabetes from 8 countries: the PREVIEW study $\mathbf{8 6}$

6.1 Introduction $\quad 86$

$\begin{array}{ll}6.2 \text { Methods } & 87\end{array}$

6.2.1 Design and analysis $\quad 87$

6.3 Results $\quad 89$

6.4 Discussion 96

6.5 Conclusion 98

6.1 Thesis Map 99

7 Study 3: Lifestyle behaviours associated with body fat percent in 9-11-yearold children, a population-based, cross-sectional study: The SportsLinx project 100

7.1 Introduction 100

$\begin{array}{ll}7.2 \text { Methods } & 101\end{array}$

7.2.1 Participants and Settings 101

$\begin{array}{lll}7.2 .2 & \text { Statistical analyses } & 102\end{array}$

$\begin{array}{ll}7.3 \text { Results } & 102\end{array}$

$\begin{array}{ll}7.4 \text { Discussion } & 104\end{array}$

$\begin{array}{ll}7.5 \text { Conclusions } & 113\end{array}$

$\begin{array}{ll}7.6 \text { Thesis Map } & 114\end{array}$

$8 \quad$ Study 4 :The effectiveness of a child weight management intervention; a mixed methods investigation of child and parent's perceptions of the behaviour change process

8.1 Background 115

$\begin{array}{ll}8.2 \text { Recruitment } & 117\end{array}$

$\begin{array}{lll}\text { 8.2.1 Schools } & 118\end{array}$

8.2.2 General Practitioners 118

8.2.3 Secondary Care 119 
$\begin{array}{lll}\text { 8.2.4 Media } & 119\end{array}$

$\begin{array}{ll}8.2 .5 & \text { General advertising } \\ 8.2 .6 & 120\end{array}$

8.2.6 Recruitment events 120

8.2.7 Results and discussion 120

8.2.8 Conclusion 122

8.3 Methods 122

8.3.1 Participants and settings 123

8.4 Results 124

8.5 Discussion 133

8.6 Summary 148

8.7 Conclusion 149

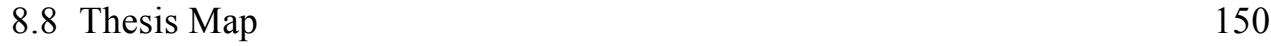

9 Summary 151

10 References $\quad 159$

11 Appendices $\quad 185$

11.1 Appendix 1 Missing data for study $1 \quad 185$

11.2Appendix 2 Missing data study $2 \quad 186$

11.3 Appendix 3 Missing data study $3 \quad 187$

11.4Appendix 4 Physical activity hand book $\quad 189$

11.5Appendix 5 Behaviour Change Toolbox 193

11.6Appendix 6 Recruitment Letter $\quad 196$

11.7Appendix 7 Radio Advert 197

11.8Appendix 8 Interview Questions 198

11.9Appendix 9 Transcriptions $\quad 200$ 


\subsection{Acknowledgments}

Sitting in front of a computer for 4 years researching the perils of sedentary behaviour and benefits of physical activity felt more than a touch ironic. At times, it was lesson in abstinence from the people and things that make me happy but it was also a great pleasure. I have been able to dedicate time and resources to my own personal, academic and professional development which is a great privilege. I am deeply grateful to the people who have made it possible and given me the support without which I would not have been able to write this thesis.

Firstly, I would like to thank my supervisor Professor Gareth Stratton for providing the opportunity, for your continual guidance, support, and friendship and of course introducing me to the beautiful Gower peninsula.

I would like to thank my second and third supervisors Dr Melitta McNarry and Dr Kelly Mackintosh. While we did not meet frequently, your advice was invaluable. Receiving your swift and expert feedback at all hours, kept me on track and working through many a late night.

I would like thank Dr Masoumeh Minou, for your invaluable contribution to the delivery and running of the PREVIEW intervention. Working alongside someone with your expertise was a great experience, it was an experience I enjoyed and one from which I learnt a great deal.

I would like to thank my colleague Dr Cain Clark. Embarking on a PhD was a daunting experience, having a baby in the middle of it, even more so. Having you as a friend and colleague, going through the same experiences, was a great help to me. I am grateful for your advice and companionship in both regards.

Finally, but most importantly, thank you to my family. Thank you mum for your love and support. Thank you Shimako for your encouragement, for looking after Mila so I could work, for your delicious cooking and for funding my trips back and forth to Wales.

Lowenna, you encouraged me to sign up to a science access course 9 years ago and you have lived with a student ever since. I am truly grateful for all you have given to support me. I dedicate this thesis to you because without your support it would not have been possible. I love you with all my heart and strive to make you proud. 


\subsection{List of Tables and Figures}

Table 1. 1 Spearman's correlation matrix of accelerometer variables

Table 1. 2 Metabolic and physical activity characteristics of adults from the

PREVIEW study

Table 1. 3 Associations between total physical activity and cardio-metabolic risk factors

Table 1. 5 Associations between Sedentary time (minutes $\cdot$ day $^{-1}$ ) and cardiometabolic

Table 2. 1 Site-Specific Descriptive Statistics for all Explanatory Variables .....90

Table 2. 2 Associations of personal and psychosocial attributes with MVPA ... 93

Table 2. 3 Moderating effects of study site age and psychosocial attributes.......94

Table 3. 1 Participant characteristics SportsLinx 103

Table 3. 2 Multi level model of associations between lifestyle behaviours and body fat percentage

Table 4. 1 Baseline characteristics of 9 participants recruited to the lifestyle intervention

Table 4. 2 Participant A

Table 4. 3 Participant B

Table 4. 4 Participant C ............................................................................ 128

Table 4. 5 Participant D ..................................................................................... 129

Table 4. 6 Changes in physical activity and sedentary time over 2 years ........ 131

Table 4. 7 Changes in HOMA-IR and Hbalc over two years ........................ 132

Table 4. 8 Changes in dietary intake over 2 years ........................................ 132

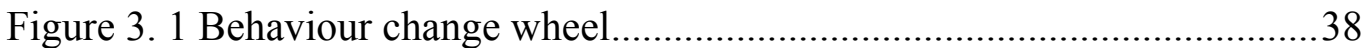

Figure 4. 1 Program design - Fading intervention ........................................65

Figure 6. 1 Moderation between social support from family and age ................95

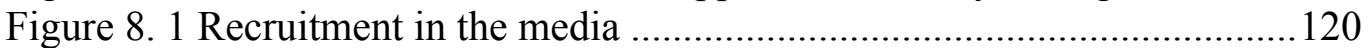

Figure 8. 2 Recruitment pathways and response rate ...................................... 121

Figure 8. 3 Changes in BMI z-score over the two-year intervention................. 125

Figure 8. 4 Participant D's exercise plan, demonstrating the use of planning and prompts

Figure 8. 5 Participant A's exercise equipment ............................................ 141

Figure 8. 6 Participants D's medals for participation in fun runs ...................... 143 


\subsection{List of abbreviations}

\begin{tabular}{|c|c|c|}
\hline Abbreviation & & Meaning \\
\hline ADA & - & American Diabetes Association \\
\hline AIDS & - & acquired immune deficiency syndrome \\
\hline ANOVA & - & Analysis of variance \\
\hline BMI & - & Body mass Index \\
\hline BMR & - & Basal Metabolic rate \\
\hline $\mathrm{BP}$ & - & Blood Pressure \\
\hline $\mathrm{CDC}$ & - & Centre of Disease Control \\
\hline CDQDPS & - & China Da Qing Diabetes Prevention Study \\
\hline CID & - & Clinical Investigation Day \\
\hline CPM & - & Counts Per Minute \\
\hline CRP & - & C-reactive protein \\
\hline CVD & - & Cardio Vascular Disease \\
\hline DESMOND & - & $\begin{array}{l}\text { Diabetes Education and Self-Management for } \\
\text { Ongoing and Newly Diagnosed }\end{array}$ \\
\hline DEXA & - & Dual-Energy X-Ray Absorptiometry \\
\hline DPP & - & Diabetes Prevention Program \\
\hline EDTA & - & Athylenediaminetetraacetic Acid \\
\hline EU & - & European Union \\
\hline FDPS & - & Finish Diabetes Prevention Study \\
\hline FPG & - & Fasting Plasma Glucose \\
\hline GI & - & Glycaemic Index \\
\hline GLUT-4 & - & Glucose transporter type 4 \\
\hline GP & - & General Practitioner \\
\hline HDL & - & High-Density Lipoprotein \\
\hline $\mathrm{HbA}_{1 \mathrm{c}}$ & - & Glycated haemoglobin A1c \\
\hline HIIT & - & High Intensity Interval Training \\
\hline HIV & - & Human immunodeficiency virus \\
\hline HOMA-IR & - & Homeostatic Model Assessment Insulin - \\
\hline
\end{tabular}




\section{Resistance}

HP

IDF

IDPP

IFG

IGT

IMD

IOTF

ISAK

ISCOLE

ITT

LCD

LDL

LGI

LPL

MET

MGI

MP

MSFT

MVPA

NHANES

NHS

NOAA

OGTT

PA

PiCRIS

PREMIT

PREPARE
- $\quad$-High Protein

- International Diabetes Federation

- $\quad$ Indian Diabetes Prevention Program

- $\quad$ Impaired Fasting Glucose

- Impaired Glucose Tolerance

- $\quad$ Indices of Multiple Deprivation

- International Obesity Task Force

International Society for the Advancement of Kinant hropometry

The International Study of Childhood Obesity,

Lifestyle and the Environment

- $\quad$ Intention to Treat

- $\quad$ Low Calorie Diet

- $\quad$ Low-Density Lipoprotein

- $\quad$ Low Glycaemic Index

- $\quad$ lipoprotein lipase

- $\quad$ Metabolic Equivalent

- $\quad$ Medium Glycaemic Index

- $\quad$ Moderate Protein

- $\quad$ Multi Stage Fitness Test

- $\quad$ Moderate-to-Vigorous Physical Activity

- $\quad$ National Health and Nutrition Examination Survey

- $\quad$ Nation health Service

- National Oceanic and Atmospheric Administration

- $\quad$ Oral Glucose Tolerance Test

- $\quad$ Physical Activity

- $\quad$ Primary Care Research Incentive Scheme

PREVIEW Behaviour Modification Intervention Toolbox

The Pre-diabetes Risk Education and Physical

- $\quad$ Activity Recommendation and Encouragement programme 
Prevention of Diabetes through Lifestyle

PREVIEW

- Intervention and Population Studies in Europe and around the World

SCT

Social Cognitive Theory

SD

- $\quad$ Standard Deviation

SDT

- $\quad$ Self-Determination Theory

SEARCH

- $\quad$ The SEARCH for Diabetes in Youth study

SES

SMART

- $\quad$ Socioeconomic Status

Specific Measurable Attainable Realistic Time bound

ST

Sedentary Time

THL

- $\quad$ National Institute for Health and Welfare, Finland

TTM

- Trans Theoretical Model

TV

- Television

UK

- $\quad$ United Kingdom

US

- $\quad$ United States

WC

- Waist Circumference

WHO

- $\quad$ World Health Organisation 


\section{Introduction}

Obesity and its comorbidities represent a global public health concern ${ }^{1}$. In 2015, the global prevalence of obesity had more than doubled since 1980, with a total of 107.7 million children and 603.7 million adults classified as obese. While the prevalence of obesity is lower among children than adults, in many countries its occurrence during childhood is rising more rapidly than in adults ${ }^{2}$. Being overweight or obese significantly increases the risk of several chronic diseases, such as cardiovascular disease (CVD), type 2 diabetes and some cancers ${ }^{3}$. Of these, CVD and type 2 diabetes comprise the two leading causes of death worldwide ${ }^{4}$. Independent of weight, physical inactivity contributes to the development of many of the world's non-communicable diseases, including type 2 diabetes, through its impact on insulin resistance ${ }^{5,6}$. Subsequently, the prevalence of type 2 diabetes has reached epidemic proportions in western countries and is growing rapidly in the developing world parallel to economic development, urbanization and the adoption of a western lifestyle ${ }^{7}$. Early onset of obesity has also led to a rise in the prevalence of type 2 diabetes among children and adolescents ${ }^{8-10}$. Worryingly, early onset type 2 diabetes (diagnosed $<45 \mathrm{yrs})$ is associated with more aggressive complications ${ }^{11}$ and, when diagnosed in youth, complications and comorbidities are higher among those with type 2 diabetes than type 1 diabetes ${ }^{12}$. Diabetes is the leading cause of $\mathrm{CVD}^{13}$, vision loss ${ }^{14}$, end-stage renal disease ${ }^{15}$ and increases the risk of cancer 16. Peripheral nerve damage can also lead to ulceration and infections which in some cases require amputation ${ }^{17}$. Consequently, diabetic complications place a huge burden on public health expenditure ${ }^{18}$. Given that the development of complications is dependent on the duration of the disease ${ }^{14,19}$ and its occurrence during childhood is increasing, it is possible that the full public health burden is yet to be realized.

Fortunately, the onset of type 2 diabetes can be delayed or prevented through lifestyle modification and weight loss. Seminal studies in this area include the US Diabetes Prevention Program (DPP) ${ }^{20}$, the Finish Diabetes Prevention Study (FDPS) ${ }^{21}$ and the China Da Qing Diabetes Prevention Study (CDQDPS) ${ }^{22}$. These randomized control trials were designed to achieve modest weight loss (5\%-7\%) through lifestyle modification to reduce the incidence of diabetes in 
people at high risk of developing type 2 diabetes. Lifestyle interventions lead to a $30 \%-58 \%$ reduction in incidence of type 2 diabetes relative to controls, providing compelling evidence for the delay or prevention of type 2 diabetes. However, the efficacy of interventions is closely related to the adherence to dietary and physical activity goals and weight loss maintenance ${ }^{21}$. Unfortunately, weight regain is common with many individuals returning to original behaviours following the removal of an intervention ${ }^{23}$. Engendering sustained behaviour change is a complex and difficult process and many overweight or obese patients experience weight regain after initial weight loss.

\subsection{Problem statement}

Physical activity is integral to lifestyle interventions, improving insulin resistance and contributing to weight loss maintenance ${ }^{24,25}$. However, previous lifestyle interventions have produced either no improvements ${ }^{26}$ or only modest improvements in physical activity in populations at high risk of type 2 diabetes 21 . Our understanding of the role of physical activity in this population is hampered by the lack of studies using objectively measured physical activity. Accelerometers are widely accepted as an objective measure of physical activity. They quantify time spent at different exercise intensities and compare favourably with doubly labelled water, the gold standard for measuring energy expenditure ${ }^{27}$. However, the extant literature is largely dependent on subjective questionnaires which have limited reliability and validity relative to laboratory measures ${ }^{28}$. Specifically, questionnaires appear to be better at detecting vigorous activity than lower intensity activity, with misclassification especially prevalent among obese individuals who are more likely to overestimate time spent in vigorous physical activity ${ }^{29}$. Therefore, it is probable that associations with light and moderate activity have been underestimated ${ }^{30}$.

Effective interventions are needed to stem the rise in diabetes developed in childhood. While lifestyle interventions in children can reduce BMI, response to weight loss interventions varies widely between participants ${ }^{31,32}$ and many that do achieve weight loss experience weight regain at later follow up ${ }^{33}$. A theoretical behaviour modification framework and behaviour change techniques are central to effective behaviour change interventions ${ }^{32}$. However, behaviour 
change techniques have largely been developed in adults. Evidence for the effectiveness of these techniques in children is inconclusive and suggests some techniques may even be detrimental to intervention effectiveness ${ }^{34}$.

On a rudimentary level, obesity prevention and management interventions need to target modifiable food and activity behaviours that influence energy balance. However, population level studies have provided little consensus as to what these are ${ }^{35,36}$. Moreover, it is not known if these behaviours changes over time as a consequence of evolving food systems and public choice.

\subsection{Thesis aims}

The overarching aims of this thesis were 1) to capture an insight into physical activity and sedentary behaviour in adults with pre-diabetes, and 2) to assess the feasibility of running a lifestyle intervention to reduce risk factors for type 2 diabetes in overweight and obese children.

Study1: The aims of this study were first to quantify physical activity, sedentary time and sleep using a $24 \mathrm{~h} \cdot \mathrm{day}^{-1}$ seven-day accelerometer protocol in adults with pre-diabetes and subsequently investigate the associations between physical activity and sedentary time with cardio-metabolic risk factors.

Study 2: The aim of this study was to assess the correlates of objectivelymeasured physical activity in adults with pre-diabetes in multiple countries and to examine inter-country differences.

Study 3: The aims of study 3 were (i) to identify associations between key food choices and lifestyle behaviours and body fat percent and (ii) determine whether these associations have remained constant over a 10 -year period in children aged 10-12 years.

Study 4: The aim of study 4 was to assess the feasibility of running a lifestyle intervention to improve risk factors for type 2 diabetes in overweight and obese children, using a descriptive multi-method case study approach to describe the behaviour change process and combine objective outcome measures with child and parent perceptions. 


\section{Literature review}

\subsection{Introduction}

Type 2 diabetes is a chronic disease characterised by high blood glucose levels (hyper glycaemia) that stem from progressive insulin resistance and a deterioration in pancreatic insulin production ${ }^{37}$. Type 2 diabetes has reached epidemic proportions in western countries, largely driven by overweight and obesity, and is growing rapidly in the developing world analogous to economic development, urbanization and the adoption of a western lifestyle ${ }^{7}$. Parallel to the rise in childhood obesity, type 2 diabetes has also become increasingly common in children and adolescents $9,10,38,39$. Despite the prevalence of type 2 diabetes rising globally, genetic predisposition means a prevalence has grown disproportionately among individuals of South Asian, African, AfricanCaribbean, Hispanic and Native American descent ${ }^{40,41}$. But although a wide variety of genetic, environmental and behavioural factors contribute to the risk of type 2 diabetes, it is widely believed to be preventable through lifestyle modification, specifically weight management, improved diet and physical activity ${ }^{7}$. The prevention of type 2 diabetes is a complex multidisciplinary issue that includes: physiology, epidemiology, genetics, pharmacology, exercise physiology, nutrition and behaviour change psychology. To comprehensively review all these areas is beyond the scope of this review. Thus the aim to summarise the literature on the prevention of type 2 diabetes, through lifestyle interventions. To set the scene, this review will outline the pathophysiological mechanisms that cause type 2 diabetes, its worldwide prevalence and its impact on healthcare systems. It will then summarise common contributors to risk with emphasis on modifiable risk factors, followed by behaviour change interventions, outlining key behaviour change concepts and highlighting the role of physical activity.

\subsection{Pathophysiology of type 2 diabetes}

Type 2 diabetes is a chronic condition resulting from the breakdown in the homeostatic feedback mechanisms that control plasma glucose concentrations ${ }^{37}$. Under normal physiological conditions a combination of tissue sensitivity to insulin and insulin secretions closely regulate plasma glucose concentrations 
during fluctuating supply and demand ${ }^{37}$. In type 2 diabetes, two abnormalities occur that disturb glucose homeostasis: (1) impaired insulin action through insulin resistance, and (2) impaired insulin secretion through a dysfunction of pancreatic $\beta$-cells ${ }^{42,43}$. The relative importance of the two abnormalities in the natural history of type 2 diabetes has received much discussion however both are present early in the disease process ${ }^{43,44}$ and progressively deteriorate during the natural history of type 2 diabetes ${ }^{45-47}$. Impaired Glucose Tolerance (IGT; Fasting plasma glucose $<7.0 \mathrm{mmol} / 1(126 \mathrm{mg} / \mathrm{dl})$ and $\geq 7.8$ and $2-\mathrm{h}$ plasma glucose $<11.1 \mathrm{mmol} / 1$ [140mg/dl and 200mg/dl]) and Impaired Fasting Glucose (IFG; Fasting plasma glucose 6.1 to $6.9 \mathrm{mmol} / 1$ [110mg/dl to $125 \mathrm{mg} / \mathrm{dl}]$ ) represent an intermediate metabolic state between normal blood glucose control and the onset of diabetes, called pre-diabetes or intermediate hyperglycaemia.

Both IGF and IFG are presentations of insulin resistance, however, the site of insulin resistance differs between the two. In individuals with isolated IFG the principal site of insulin resistance is the liver, with close to normal values in skeletal muscle. Conversely, isolated IGT is characterised by peripheral insulin resistance with near normal hepatic insulin resistance ${ }^{48,49}$. While the alternative definitions have distinct abnormalities, they represent an increased risk of developing overt diabetes with an annualised incident rate for isolated IGT of 4$6 \%$ ) and isolated IFG of $6-9 \%$. Combined, the annualised incident rate is $15-$ $19 \%{ }^{50}$.

Glycated haemoglobin (HbAlc) is formed through the binding of haemoglobin and glucose within erythrocytes. The rate of formation is directly proportional to glucose concentrations and provides an indirect measure of average plasma glucose over the life span of an erythrocyte, roughly 120 days ${ }^{51}$. The American Diabetes Association introduced an $\mathrm{HbA1c}$ level of 5.7-6.4\% as an additional threshold for increased pre-diabetes ${ }^{52}$. The use of HbAlc for the diagnosis of type 2 diabetes is becoming standard clinical practice in many countries and is more convenient than glucose testing as it does not require fasting, timed samples, and it is not affected by changes in diet or physical activity. The rate of development from pre-diabetes to overt diabetes based on the HbA1c threshold is broadly similar to IGT and IFG ${ }^{49}$. However, some debate remains over the use of HbAlc when compared with standard OGTT as a diagnostic test ${ }^{53}$. 
Overt diabetes is defined by the International Diabetes Federation (IDF) and the World Health Organization (WHO) as a fasting plasma glucose $\geq 7.0 \mathrm{mmol} / 1$ $(126 \mathrm{mg} / \mathrm{dl})$ or $2-\mathrm{h}$ plasma glucose $\geq 11.1 \mathrm{mmol} / 1(200 \mathrm{mg} / \mathrm{dl})$ two hours after ingestion of $75 \mathrm{~g}$ oral glucose load ${ }^{54}$.

Alternatively, Hbalc $\geq 6.5 \%$ is also widely used as a diagnostic criteria for type 2 diabetes 52 .

\subsection{Prevalence}

\subsubsection{Adults}

The global prevalence of diabetes was estimated at 415 million cases $(8.8 \%)$ in $2015^{55}$ and all projections suggest this will continue to rise in the coming decade: 552 million by $2030^{56} ; 592$ million by $2035^{57} ; 642$ million by $2040^{55}$. Middle and higher income countries have a greater prevalence than low income countries with the highest age adjusted prevalence found in North America and the Caribbean at $11.5 \%$ in $2015^{55,57}$. However, middle and lower income countries have seen the greatest increase in prevalence in the last 30 years with countries such as Indonesia, Pakistan, and Mexico replacing European countries, including Germany, Italy, and the UK in the top ten countries, with most adults with diabetes ${ }^{58}$. Furthermore, low income countries are predicted to see the greatest increases in the coming years ${ }^{55-57}$ in what has truly become a global health problem. Although not all studies of diabetes prevalence have distinguished between the type of diabetes, those that have, suggest $87-91 \%$ of all people with diabetes have got type 2 diabetes ${ }^{59-61}$.

\subsubsection{Children}

Parallel to the rise in childhood obesity, type 2 diabetes has become increasingly common in children and adolescents ${ }^{9,10,38,39}$. In the US, the SEARCH study ${ }^{9}$ recorded cases of diabetes annually from a registry of 5.5 million children $<20$ years of age. The prevalence of type 2 diabetes increased by $30.5 \%$ between 2001 and 2009 to 0.46 per 1,000 in 2009. Increases were observed in both sexes and among Hispanic and non-Hispanic white and African American youth. However, prevalence was greater among American Indian (1.20 per 1,000), black (1.06 per 1000), and Hispanic youth (0.79 per 1000$)$ compared to white youth $(0.17 \text { per } 1,000)^{9}$. 
Given the progressive nature of the two underlying abnormalities that lead to type 2 diabetes (IGT and IFG), the risk of developing type 2 diabetes increases with age. What was once called Adult Onset Diabetes is the leading cause of new cases of diabetes in children and adolescents from Japan and Taiwan ${ }^{39}$. In Japan, the prevalence increased 10-fold between 1976 and $1995^{39}$. Increases in prevalence have been found in New Zealand ${ }^{62}$, Australia ${ }^{63}$, Germany ${ }^{64,65}$, Hungary and Canada ${ }^{66}$ while cases have been widely reported over the world ${ }^{39}$. In the UK, three studies have examined the incidence or prevalence of type 2 diabetes in children and adolescent in the last two decades ${ }^{10,67,68}$. In 2004, a survey of paediatric diabetes centres reported a prevalence in children $<16$ years of $0.21 / 100,000$ with a significantly higher risk among ethnic minorities ${ }^{68}$. In 2007, Haines et al ${ }^{10}$ in a prospective monthly surveillance of 2,665 consultant paediatricians in the U.K, found the incidence of type 2 diabetes in children $<17$ years was $0.53 \cdot 100,000^{-1} \cdot$ year $^{-1}$. In 2009 , a retrospective cohort study covering 8 years, reported significant increase in prescription of antidiabetic drugs in children and adolescents aged $0-18$ years ${ }^{67}$, indicating that the prevalence of both type 1 and type 2 diabetes are rising rapidly. Cautious estimates of type 2 diabetes were $1.9 \cdot 100,000$, notably higher than previous estimates ${ }^{68}$ and are likely to be an underestimate owing to undiagnosed cases and those controlled with diet alone.

Although the overall prevalence of type 2 diabetes remains low relative to adults, the intermediate metabolic condition IGT is frequently reported in obese children with reports ranging from $15 \%$ to $25 \%{ }^{69,70}$ and $36.3 \%$ in obese children with at least one other risk factor, such as a positive family history ${ }^{8}$. Comparatively, IFG is less frequent in children without type 2 diabetes $(1.7 \%)^{71}$ but is present in children with the type 2 diabetes thus is symptomatic of a more advanced stage of diabetes and not a sensitive predictor of future impaired glucose regulation ${ }^{70}$.

Although not all children with IGT develop type 2 diabetes, the rate of conversion appears to occur more quickly in children than adults, in as little as 12-21 months ${ }^{72}$. A prospective study that followed 117 obese adolescents for 2 years showed that $8 \%$ of those with IGT progressed to type 2 diabetes, $45.5 \%$ returned to normal glucose tolerance and $30.3 \%$ remained IGT. Severity of obesity, higher baseline IGT and ethnicity were predictive of progression to type 
2 diabetes while a reduction in BMI predicted a return to normal glucose tolerance ${ }^{72}$. In a similar study, that followed 128 white, obese children and adolescents, $75 \%$ returned to normal glucose tolerance, 16\% remained IGT, 2\% developed type 2 diabetes. Higher baseline 2-h glucose and weight gain predicted developing type 2 diabetes. Similarly, Jaruratanasirikul and colleagues $^{73}$, followed 177 obese children and adolescents for 3 to 6 years. During follow-up, $14.4 \%$ developed type 2 diabetes, weight gain, IGT, BMI, FPG and HOMA-IR were predictive of developing type 2 diabetes ${ }^{73}$. While many children with IGT return to normal glucose tolerance, progression from IGT to type 2 diabetes is closely related to obesity and weight gain. Consequently, the growing prevalence of childhood obesity is paralleled by a rise in type 2 diabetes among children and adolescents ${ }^{8-10}$.

\subsection{Impact of type 2 diabetes}

\subsubsection{Mortality}

According to the International Diabetes Federation (IDF), diabetes accounted for $10.7 \%$ of global all-cause mortality among people aged between 20 and 79 years in 2017. Approximately 4 million people are estimated to have died from diabetes, and $46.1 \%$ of deaths occurred in adults under the age of 60 years. This is higher than the combined number of deaths from HIV/AID, tuberculosis and malaria in $2015^{17}$. The World Health Organisation (WHO) report on diabetes in 2016 stated 1.5 million deaths were caused by diabetes in 2012 but a further 2.2 million deaths were caused by higher than optimal blood glucose levels increasing the risks of cardiovascular disease (CVD) ${ }^{74}$. Diabetes-related complications are caused by persistently raised glucose concentrations, which impair the microcirculation and can affect the heart, eyes, kidneys and nerves. The subsequent tissue hypoxia leads to complications that are specific to that organ ${ }^{75}$. Diabetes related complications are commonly placed into two categories (1) microvascular such as nerve damage (neuropathy), renal system damage (nephropathy) and eye damage (retinopathy), and (2) macrovascular complications including cardiovascular disease, stroke, and peripheral vascular disease. 


\subsubsection{Morbidity}

Diabetes is the leading cause of CVD $^{13}$ which in turn causes the greatest number of deaths globally ${ }^{76}$. A recent report by the IDF revealed that among adults with diabetes from high and middle-income countries and between the age of 56 and 66 years, the prevalence of CVD ranged from $14.8 \%$ to $40.5 \%{ }^{13}$. Retinopathy is caused by damage to the retinal capillaries, which can lead to capillary leakage and capillary blockage and eventually loss of vision and blindness ${ }^{14,77}$. Retinopathy affects approximately one in three people living with diabetes and is the leading cause of vision loss in working-age adults ${ }^{14}$. Diabetic nephropathy is the leading cause of end-stage renal disease accounting for approximately $50 \%$ of all cases in the developed world ${ }^{15}$. Diabetic neuropathy consists of damage to the nerves throughout the body and can impair autonomic, motor and sensory functions. Complications include ulceration, serious infections and in some cases amputations ${ }^{17}$. Although the prevalence varies by country, globally $6.4 \%$ of patients with type 2 diabetes will experience foot ulcerations ${ }^{19}$ and lower limb amputations are $>20 \%$ more likely in those with diabetes ${ }^{78}$. Numerous meta-analyses have also shown increased specific cancer risk among individuals with diabetes including breast cancer ${ }^{79}$, pancreatic cancer $^{80}$, liver cancer ${ }^{81}$ as well as overall cancer risk ${ }^{16}$.

\subsubsection{Financial}

Due the numerous complications and escalating prevalence, type 2 diabetes imposes a substantial financial burden on health care systems. In the US, the medical costs of treating type 2 diabetes and diabetic complications in newly diagnosed patients, weighted for age and sex, was $\$ 85,200$ of which 53\% was due to treating diabetic complications ${ }^{82}$. The total economic cost of diabetes in the US (type 1 and type 2) in 2012 was $\$ 245$ billion ${ }^{83}$. In the UK, in 2010/2011 type 2 diabetes cost $£ 8.8 \mathrm{bn}$ in direct medical costs and a further $£ 13 \mathrm{bn}$ in indirect costs such as social and economic productivity costs ${ }^{18}$. The total cost of diabetes to the NHS accounts for approximately $10 \%$ of the total health resource expenditure and if current trends continue, is projected to account for $17 \%$ by $2035^{18}$. 
Given that the development of diabetic complications is dependent on the duration of the disease ${ }^{1419}$ it is possible that the full public health burden of developing type 2 diabetes during childhood has not yet been realised. Adults with early onset type 2 diabetes (diagnosed between 18-44yrs) experience more aggressive complications than usual onset type 2 diabetes (diagnosed $>45 y$ rs); they are more likely to start insulin therapy, suffer from microalbuminuria and experience macrovascular complications such as myocardial infarction ${ }^{11}$. Long term follow up studies in children are lacking. However, a lifetime exposure to hyperglycaemia may cause a higher risk of complications over time ${ }^{84}$. Predictive models by Rhodes et al. ${ }^{85}$ suggest adolescents with type 2 diabetes may lose approximately 15 years from average remaining life expectancy and may experience severe, chronic complications of type 2 diabetes by their $40 \mathrm{~s}^{85}$.

\subsection{Risk Factors}

The risk of developing type 2 diabetes is increased by a number of both modifiable and non-modifiable risk factors.

\subsubsection{Non-modifiable risk factors}

The global prevalence of type 2 diabetes is greater among men than women ${ }^{58}$ with men developing the disease at an earlier age and lower BMI ${ }^{86}$. Given the progressive nature of the underlying pathology of type 2 diabetes (IGT and IFG), the risk of developing type 2 diabetes increases with age and most people with type 2 diabetes are above the age of 40 years ${ }^{56}$. Large prospective cohort studies have shown that a family history of diabetes is associated with greater incidence of diabetes compared with those without a family history of diabetes ${ }^{87-89}$. In a study of 2,527 descendants from 1,303 families in the Framingham offspring study ${ }^{87}$, the age-adjusted Odds Ratio for offspring with type 2 diabetes was 3.4 among individuals with maternal diabetes, 3.5 among individuals with paternal diabetes and 6.1 among individuals with both maternal and paternal diabetes ${ }^{87}$. In the Nurses' Health Study ${ }^{88}$ of 73,227 women, having at least one first degree relative with diabetes was associated with a Relative Risk of 2.27. Moreover, participants with a family history of diabetes also had a higher BMI, which explained $21 \%$ of the association between family history of diabetes and risk of type 2 diabetes. This association was further explained by dietary factors such as the intake of red meat, alcohol, and sugar-sweetened beverages reflecting that family history indicates a shared environment as well as genotype. In contrast, in 
a Japanese study of 3,517 participants, the Hazard Ratio of developing type 2 diabetes over the 7-year study period was 1.82 in participants with a family history of diabetes relative to those without a family history of diabetes ${ }^{89}$. The Hazard Ratio was not attenuated after controlling BMI and lifestyle factors. Genome-wide association studies have identified multiple common risk alleles and genomic loci at which common variants influence type 2 diabetes ${ }^{90,91}$. The Diabetes Genetics Replication and Meta-Analysis Consortium identified more than 50 common variants for type 2 diabetes ${ }^{92}$. However, in a systematic review, cumulative genetic risk scores calculated by summing the number of diabetes risk alleles did not improve the prediction of type 2 diabetes beyond traditional risk factors ${ }^{93}$. Analysis of the Nurses' Health Study and the Health Professionals Follow-up Study suggest the association between genotype and type 2 diabetes is mediated by a predisposition for central obesity which is in turn a prominent risk factor for type 2 diabetes ${ }^{94}$. Interactions between lifestyle factors and genetic predisposition have also been observed in the Health Professionals Follow-up Study where a greater association between a western diet and type 2 diabetes was found in those with a higher cumulative genetic risk score proposing an interaction between genetic predisposition and environment 95 .

Large disparities exist between ethnic groups in the prevalence of type 2 diabetes and the development of diabetes related complications ${ }^{96-98}$. In a study of 46,091 adults from California US, after controlling for lifestyle factors, the highest prevalence of type 2 diabetes was reported among Native Americans (32.4\%), Filipinos (15.8\%), and Japanese (11.8\%) compared to Caucasians $(6.1 \%)^{99}$. Similarly, in 2017 the Centre for Disease Control (CDC) reported that the prevalence among American Indians/Alaska Natives was (15.1\%), nonHispanic blacks (12.7\%), and people of Hispanic ethnicity (12.1\%) and nonHispanic whites $(7.4 \%)$ and Asians $(8.0 \%)^{41}$. In Europe, type 2 diabetes is up to six times more common in people of South Asian descent and up to three times more common among those of African and African-Caribbean origin ${ }^{40}$. 


\subsubsection{Modifiable risk factors}

While age, sex, family history and ethnicity provide a genetic predisposition to the development of type 2 diabetes, lifestyle factors appear to be the main cause of the dramatic rise in prevalence in recent decades. The strongest modifiable risk factor for type 2 diabetes is overweight and obesity ${ }^{100}$. Both cross-sectional 101,102 and prospective cohort studies ${ }^{100,103,104}$ have consistently reported strong positive association between BMI and type 2 diabetes and the risk appears to increase exponentially with an increase in BMI ${ }^{104}$. Although BMI is typically used as a measure of adiposity in observational studies, growing evidence suggests that body fat distribution, specifically central or abdominal obesity, may provide a better measure of risk than BMI. Anthropometric measures capturing abdominal adiposity, such as waist circumference and waist-to-hip ratio, have been related to diabetes risk independent of BMI 105106 . It is postulated that the accumulation of visceral fat contributes to growth and proliferation of adipocyte and the secretion of signalling molecules (adipokines) which promote and exacerbate insulin resistance ${ }^{107}$. Nonetheless, two recent meta-analyses were unable to separate the strength of association or Odds Ratios between type 2 diabetes and waist circumference, waist-to-hip ratio or BMI 106,108 . Weight change also has a bearing on the risk of type 2 diabetes, weight gain is associated with a subsequent increase in risk in both healthy weight ${ }^{109,110}$ and overweight adults ${ }^{111}$, consequently avoiding weight gain even in lean individuals is important to reduce the risk of disease. Inversely, even modest reductions in weight are associated with a reduction in risk ${ }^{109}$. Weight cycling through repeated, unsuccessful attempts at maintaining weight loss has also been proposed as an independent risk factor for type 2 diabetes ${ }^{112,113}$ however reports have been inconsistent ${ }^{114,115}$.

\subsubsection{Diet}

The type 2 diabetes epidemic is largely driven by overweight and obesity which is widespread in developed countries and growing rapidly in the developing world analogous to economic development, urbanization and the adoption of a western lifestyle ${ }^{7}$. Global food systems are producing an abundance of 
processed and affordable foods supportive of passive energy overconsumption 116

In recent years, many systematic reviews and meta-analyses of large crosssectional and prospective cohort studies as well as clinical trials, have emerged to support the role of individual nutrients, foods, and dietary patterns in the pathogenesis of type 2 diabetes 117,118,127-133,119-126. The quality of carbohydrate intake, specifically cereal fibre intake and a high glycaemic index and glycaemic load, are associated with an increased risk of type 2 diabetes and support a diet rich in minimally processed whole grains and low GI ${ }^{117,118}$. These findings are supported in a recent clinical trial which showed that a lower glycaemic index diet consumed ad libitum, produced significantly better weight loss retention. Moreover, participants were twice as likely to have maintained a 5\% weight loss compared to control groups ${ }^{119}$.

The role of dietary fat in the development of type 2 diabetes is inconsistent. While studies replacing saturated fats and trans fatty acids with unsaturated fats have shown beneficial effects on insulin sensitivity ${ }^{121,122}$, two recent metaanalyses found no association between total, saturated, trans, unsaturated and long-chain n-3 fatty acid intake and risk of type 2 diabetes ${ }^{134,135}$. However, the type of fat seems to have an important effect; linoleic acid improves insulin resistance ${ }^{121}$ and has a protective effect against the risk of type 2 diabetes; an increase from 3 to $6 \%$ of total energy has been associated with a $20 \%$ reduction in risk ${ }^{135}$. Despite only modest associations with type 2 diabetes outcomes, dietary fat does have a strong association with body fat. A meta-analysis of 33 randomised controlled trials and 10 cohort studies of durations from six months to eight years, showed consistently that lower total fat intake produced sustained reductions in body weight ${ }^{120}$. Similarly, in the Finish Diabetes Prevention Study dietary fat and fibre intake were significant predictors of sustained weight reduction and progression to type 2 diabetes ${ }^{136}$. The source of dietary fats may also be of importance, a meta-analysis of prospective cohort studies found inverse associations between dairy intake and type 2 diabetes, particularly low fat and fermented dairy products ${ }^{137}$. Similarly, a systematic review found that high-fat dairy consumption did not contribute to obesity risk ${ }^{138}$. The consumption of red and processed meats has consistently been associated with increased risk of diabetes ${ }^{123,124}$. In a meta-analysis of the Health Professionals 
Follow Up Study and the Nurses' Health Study ${ }^{124}$, the substitutions of one serving of red meat per day for nuts, low-fat dairy or whole grains was associated with a $16-35 \%$ lower risk of type 2 diabetes ${ }^{124}$.

Greater intake of sweetened soft drinks has also been associated increased risk of type 2 diabetes in a number of meta-analyses ${ }^{125-127}$. The associations were either not attenuated or only slightly lower in studies adjusting for BMI suggesting the association is only partially mediated by BMI and implies other mechanisms are involved. Furthermore, artificially sweetened soft drinks and fruit juice are also positively associated with incidence of type 2 diabetes so do not provide a healthy alternative to sweetened soft drinks and suggest the calorie content is not responsible for the observed association.

Between 2010 and 2016, six meta-analyses have been published collating the evidence for associations between fruit and vegetable consumption and the risk of type 2 diabetes; all concur that the consumption of fruit and vegetables is inversely associated with type 2 diabetes risk ${ }^{128-133}$. Specifically, green leafy vegetables ${ }^{128-130}$, root vegetables ${ }^{131}$, berries and yellow vegetables ${ }^{130}$ are associated with a protective effect.

Moderate alcohol consumption also appears to have a protective effect on diabetes risk with a U-shaped relationship; moderate alcohol intake is associated with a reduced risk relative to non-drinkers, however, a high intake is associated with an increased risk ${ }^{139}$. In a more recent meta-analysis these associations were confined to women and non-Asian populations ${ }^{140}$.

Similarly, coffee and tea consumption are associated with lower incidence of type 2 diabetes with a dose response relationship that remains constant for caffeinated, decaffeinated instant coffee and tea ${ }^{141-143}$.

While systematic reviews and meta-analyses of prospective cohort studies provide compelling evidence for individual foods and nutrients, foods are not consumed in isolation but rather in combinations. To estimate a person's adherence to a set diet or recommended intake, several indices have been developed that produce an a priori assessment of dietary patterns. In a metaanalysis of dietary patterns, the adherence to three diets was associated with significant risk reductions of incident diabetes. First, the Mediterranean diet consisting of fruits, nuts, vegetables, legumes, fish and seafood, moderate alcohol consumption, no refined grains and olive oil. Second, the Dietary 
Approaches to Stop Hypertension composed from 10 food groups: grains; highfibre grains, vegetables, fruits, total dairy, low-fat dairy, nuts, seeds, and legumes, meat, fats, oil, and sweets. Finally, the Alternative Healthy Eating Index, including whole grains, vegetables, fruits, and dairy, the white to red meat ratio, trans fat, and the ratio of poly unsaturated to saturated fatty acids. Despite partial variation between diets, all had considerable overlap of desirable food groups. In an alternative evaluation using principle component analysis, a western dietary pattern characterized by red and processed meat, refined grains, high-fat dairy, eggs, and fried products were positively associated with diabetes, whereas, patterns characterized by vegetables, legumes, fruits, poultry, and fish were inversely associated with diabetes ${ }^{144}$.

\subsubsection{Physical activity}

Physical inactivity has a major impact on the development of the worlds noncommunicable diseases including type 2 diabetes ${ }^{5}$. It has been shown empirically, that higher levels of physical activity lead to reduced risk of obesity and type 2 diabetes ${ }^{145,146}$. In recent meta-analyses of prospective cohort studies, physical activity of all intensities (light, moderate, vigorous and total) and subgroups (leisure-time activity, occupational activity and resistance exercise) were inversely associated with risk of type 2 diabetes in a dose response manner 146. Physical activity plays an important role, independent of weight, in the prevention of type 2 diabetes through its effect on insulin resistance, a major risk factor for the development of type 2 diabetes ${ }^{6}$. Invers associations between physical activity and insulin resistance have been reported in healthy lean adults ${ }^{147}$, adults with high risk of diabetes ${ }^{148}$, and children and adolescents ${ }^{149}$. Physical activity improves insulin sensitivity by stimulating the translocation of GLUT-4 transporters to the plasma membrane increasing glucose uptake into skeletal muscle ${ }^{150}$. A single bout of physical activity, be it aerobic or resistance exercise, can elicit a significant increase in insulin sensitivity for up to 48 hours. Equally, exercise training can produce significant improvements in insulin sensitivity independent of body weight or body fat. However, improvements are transient and gradually declining if activity is not maintained ${ }^{151}$. 


\subsubsection{Physical activity intensity}

Prospective cohort studies consistently show physical activity to reduce the risk of type 2 diabetes ${ }^{100}$. However, several studies have failed to find an association for light to moderate intensity activities such as walking ${ }^{152,153}$ which raised the question of whether a minimum intensity threshold is required to elicit the benefits of physical activity. On the balance of two recent meta-analyses ${ }^{146,154}$, physical activity appears to confer a reduction in the risk of type 2 diabetes at all intensities. In a meta-analysis of prospective studies and randomised control trials, the Relative Risk of incidence of diabetes was $0.61,0.68$ and 0.66 for vigorous, moderate and low intensity activity respectively, suggesting that all intensities are beneficial ${ }^{146}$. In a more recent meta-analysis, when physical activity was measured as metabolic equivalents (METs; one MET being equal to the energy cost of a person during quiet sitting ${ }^{154}$ ), achieving an 11.25 MET $\mathrm{h} /$ week, which is equivalent to $150 \mathrm{~min} /$ week of moderate activity, was associated with $26 \%$ reduction in risk, relative to inactive individuals ${ }^{155}$. However, the magnitude of risk reduction was greater at higher intensity and diminished at lower intensity suggesting that higher intensity leads to greater risk reduction.

Physical activity is an independent risk factor for type 2 diabetes however associations are attenuated by BMI suggesting that the effect of physical activity is in part mediated through impact on BMI ${ }^{156}$. Interestingly, several studies have shown the effect of low intensity activity, including walking, is attenuated to a greater extent than vigorous activity ${ }^{152,157}$. This may suggest that the effect low intensity activity is mediated by changes in BMI whereas vigorous activity has a more direct metabolic effect. Improvements in insulin sensitivity can be achieved at a range of exercise intensities and volumes but the magnitude of change appears to be related to the duration and intensity of exercise. Even light intensity activity (1.1-2.9 METS) can improve insulin sensitivity ${ }^{158}$ however greater effects are seen with higher intensity and prolonged physical activity ${ }^{159}$. In a randomised control trial of overweight/obese sedentary subjects, Houmard et al. ${ }^{160}$ showed that physical activity improved insulin sensitivity at all intensities and volumes compared to controls. However, exercise duration had the greatest effect on insulin sensitivity regardless of intensity or volume, thus they concluded that duration was the most important factor in improving insulin 
sensitivity. In contrast, a systematic review and meta-analysis of high intensity interval training (HIIT) showed that high intensity intervals are also an effective method of improving insulin resistance comparing favourabley to continuous training and controls ${ }^{161}$.

In summary, physical activity reduces the risk of type 2 diabetes at a range of intensities with more pronounced effects occurring at higher intensities or greater volume.

\subsubsection{Sedentary time}

Sedentary behaviour i.e. behaviours that require low energy expenditure such as TV viewing, seated desk work and driving, have a negative effect on cardiometabolic health independent of both BMI and MVPA ${ }^{148,162}$. Recent metaanalyses of prospective cohort studies have shown that higher daily sedentary time is associated with greater risk of all-cause mortality ${ }^{163}$, type 2 diabetes and cardiovascular disease ${ }^{164}$. In adults with pre-diabetes, sedentary time is associated with poor glycaemic control, triglycerides, HDL-cholesterol, waist circumference, and Homeostatic Model Assessment of Insulin Resistance (HOMA-IR) ${ }^{148,165}$. Sedentary time is thought to affect glucose homeostasis and lipid metabolism by reducing muscle GLUT-4 content and insulin-stimulated glucose uptake ${ }^{166}$ while also reducing lipoprotein lipase (LPL) activity leading to impaired triglyceride and HDL cholesterol metabolism ${ }^{167}$.

\subsubsection{Metabolic syndrome}

The metabolic syndrome is a collection of cardio-metabolic abnormalities that include central obesity, insulin resistance, dyslipidaemia, hypertension and a pro-inflammatory state ${ }^{168,169}$.

Although the precise definition and diagnostic criteria varies by organization, the World Health Organization, International Diabetes Federation, European Group for the Study of Insulin Resistance, National Cholesterol Education ProgramThird Adult Treatment Panel and the American Association for Clinical Endocrinology, recognise the metabolic syndrome as a useful clinical construct that indicates a substantial increase in the risk of cardiovascular disease and type 2 diabetes ${ }^{169-171}$. Obesity and insulin resistance are both central to the metabolic 
syndrome and have both been proposed as causative factors ${ }^{172,173}$. Insulin resistance and subsequent hyperinsulinemia has been shown to precede the development of other aspects of the syndrome ${ }^{174}$, abnormalities appear to cluster around the presence of insulin resistance with the severity of insulin resistance being associated with a greater number of metabolic abnormalities 175. Obesity, especially central obesity and visceral fat, contributes to hyperglycaemia, hypertension, high serum triglycerides, low HDL-cholesterol and is the prominent risk factor for insulin resistance ${ }^{172,176}$. A Suggested mechanism is the secretion of cell signalling molecules from adipose tissue (adipokines) resulting in localized and systemic inflammation mediating many of the comorbidities related to obesity ${ }^{176}$.

Despite strong evidence for the role of physical activity and sedentary time in the development of type 2 diabetes and components of the metabolic syndrome (cardio-metabolic risk factors), many studies reporting associations between physical activity and sedentary and cardio-metabolic risk factors are limited by the self-reported measures of physical activity and sedentary time ${ }^{177,178}$ which are susceptible to reporting and recall bias with over reporting prevalent among overweight and obese individuals ${ }^{179}$. In the limited number that have used objective measures of physical activity, investigations of international samples measured concurrently with the same protocol are lacking, little consistency exists with regards to the devices, wear time criteria and intensity cut points used. Specifically, accelerometer cut points used to define intensity thresholds are limited to the population from which they were derived ${ }^{180}$. Typically, calibration studies align the movement captured by the accelerometer with the energy cost quantified by indirect calorimetry ${ }^{181}$. Metabolic equivalents are used to provide intensity categories. Commonly 3-5.9 METs is moderate activity, 68.9 METs is vigorous, and $>9$ METs is very vigorous ${ }^{182}$. However, large disparities exist between different cut points derived in this manner. Consequently, the prevalence of meeting PA guidelines is largely influenced by which cut points are used ${ }^{181,183,184}$. Studies have suggested that cut points derived from younger adults overestimate the energy cost of older adults when used with this population ${ }^{185}$. Whitcher and Popadopoulos ${ }^{186}$ reported a significant difference in relative oxygen consumption and MET values at rest and during all exercise intensities between young and older adults. 
Consequently, moderate intensity physical activity for older adults occurs at lower accelerometer derived activity counts than younger adults. Furthermore, in overweight and obese individuals, due to a greater body mass, energy expenditure during rest and exercise is higher compared to healthy weight individuals ${ }^{187}$. Unless this is accounted for, time spent at different exercise intensities is likely to be underestimated. This uncertainty surrounding the application of accelerometer cut points from one population to another limits the ability to make comparisons between studies.

Despite those with pre-diabetes being at the highest risk of developing type 2 diabetes and representing the population at which many lifestyle interventions are targeted, few studies have described the relationship between physical activity and insulin resistance in this population. Typically, epidemiological research assessing the effect of physical activity and sedentary time on cardiometabolic risk factors has been conducted in the general population ${ }^{188-190}$. The few studies conducted in high risk populations have included participants based on a risk score questionnaire ${ }^{148}$, family history of type 2 diabetes ${ }^{177,191}$ or participants diagnosed with diabetes and pre-diabetes have been combined ${ }^{192}$. Consequently, physical characteristic and metabolic parameters of study participants varied substantially. As a result, it is unclear to what extent reported associations could be inferred to individuals with pre-diabetes.

\subsection{Correlates of physical activity}

While the potential benefits of physical activity in populations at risk of diabetes are great, most patients with type 2 diabetes, or at highest risk for developing type 2 diabetes, do not engage in sufficient physical activity to elicit these associated health benefits ${ }^{193}$. Even among lifestyle interventions many fail to meet their physical activity targets or significantly increase their physical activity levels ${ }^{194-196}$. The promotion of physical activity in this population depends on the understanding of the underlying influential factors, and consequently theory, which improve the likelihood of achieving the desired behavioural change.

Systematic reviews of correlates and determinants of physical activity have identified a diverse range of factors that influence physical activity ${ }^{197,198}$. 
Ecological models of health behaviour specify the combination of multiple levels of influence on behaviour, combining individual, social, environmental and policy level factors ${ }^{199}$. Variables within individuals (intra-personal) including age (inverse), sex, socioeconomic status, level of education, occupational status and BMI (inverse) are among the most consistently reported correlates of physical activity ${ }^{197,198}$. Psychosocial factors are also widely studied, of which self-efficacy is the most consistently reported and is associated with physical activity participation, initiation and adherence ${ }^{197,200}$. Intention to exercise, and barriers to physical activity (lack of time, too tiring, fear of falling, bad weather, lack of facilities) are also frequently reported correlates of physical activity ${ }^{197,198}$. Inter-personal variables such as social support from family, friends and work colleagues and cultural norms have also been show to correlate with physical activity ${ }^{197,198}$. At the environmental level, neighbourhood design aspects such as walkability, footpaths, pavements, neighbourhood safety, access to recreation facilities, transportation environment, aesthetics, and observation of others engaging in physical activity (modelling), have all been shown to correlate with physical activity. However, little consistency exists between studies of environmental correlates of physical activity ${ }^{197,198,201}$.

Previous research has also shown that correlates of physical activity differ between country ${ }^{202,203}$. For example, in American, Australian and European studies physical activity declines with age ${ }^{197,198}$. In contrast, in Asian countries physical activity is positively associated with age largely due to increased leisure time physical activity after retirement ${ }^{204,205}$. Younger adults may also be less active due to time spent working. In a study of Taiwanese adults, long work hours and family responsibilities were the most frequent perceived barriers to physical activity ${ }^{205}$.

Although self-efficacy is largely considered to be a universal construct ${ }^{206}$, and has been shown to predict physical activity in a range of countries ${ }^{207}$, in a study of 6,014 adults from three countries (Australia, USA and Belgium) Van Dyke and colleagues ${ }^{202}$ demonstrated that associations between physical activity selfefficacy, as well as enjoyment, perceived barriers, perceived benefits, and perceived social support from friends, were site specific ${ }^{202}$. Therefore, it is possible that some predictors have a more or less important role in differing cultural or environmental contexts. In a comparison of American and Taiwanese 
adolescents, perceived self-efficacy directly predicted physical activity in the Taiwanese participants. However, in American participants, perceived selfefficacy predicted physical activity only by mediating beliefs about exercise benefits and barriers ${ }^{208}$. This shows a differing mechanism behind the associations between physical activity and self-efficacy depending on cultural context.

In a comparison of psychosocial and environmental correlates of physical activity between adults from a northern and southern European country (Belgium and Portugal), ${ }^{203}$ the ease of accessing public transport was positively associated with active transport, leisure time, activity, MVPA and total activity in the Belgian sample but this was only the case for active transport in the Portuguese sample. In Portugal, the availability of sidewalks was associated with walking during leisure time. This demonstrates that environmental correlates of walking vary by country but also that they differ depending on whether the walking is performed for pleasure or for transport. Interestingly, while total minutes spent in active transport were comparable between the two countries, more walking was reported in Portugal while more cycling was reported in Belgium. Walkability/cycleability was related to active transport in Belgium but did not explain additional variance beyond psychosocial variables. Specifically, in Belgium, modelling behaviour was predictive of active transport while in Portugal, social support from friends was significantly associated with active transport. Further comparison between the two countries reveals that the Belgian sample perceived engaging in physical activity to be more fun than the Portuguese, engaging in activity for its inherent pleasure rather than external reward. This suggest a more intrinsic motivation which, from the perspective of self-determination theory, is associated with greater adherence to health behaviours including physical activity ${ }^{209}$.

These studies demonstrate that at the individual, social and environmental level, some correlates of physical activity have a more or less important role depending on cultural or environmental contexts. Therefore, despite the global nature of the diabetes epidemic, a universal approach to physical activity promotion may not be appropriate. Due to inter-study differences in methodological approaches, questionnaires and tools used to assess the psychosocial attributes and environmental correlates of physical activity, the 
ability to draw comparisons between studies is limited. To date, very few studies have compared psychosocial correlates across countries limiting our understanding of these interactions.

The majority of research on correlates and determinants of physical activity has focussed on the general population. Interventions in overweight and obese adults have confirmed variables such as social-support, self-efficacy, autonomy and self-monitoring as important predictors of physical activity ${ }^{197}$. However, BMI has also been shown to moderate associations between social support, selfefficacy and availability of facilities ${ }^{201}$. Given that BMI, is a potent risk factor for type 2 it is postulated that individuals at high-risk of type 2 diabetes, may have different underlying facilitators and barriers to physical activity. Moreover, the interpretation of the limited research conducted in adults at risk of type 2 diabetes ${ }^{210,211}$ is severely limited by the reliance on self-reported physical activity, which is inherently susceptible to subjective interpretation and over reporting in overweight/obese individuals ${ }^{179}$.

\subsection{Behaviour change}

Weight loss has significant positive effects on body composition, insulin sensitivity and risk of type 2 diabetes ${ }^{212} 109$. However, these benefits are only sustained with weight loss maintenance. Weight regain is a significant problem following initial weight loss with many individuals returning to original behaviour following the removal of an intervention. Indeed, a systematic review of long term weight loss interventions found that almost half of the initial weight loss was regained after 1-year ${ }^{23}$. Clearly, long-term behaviour change is required for weight loss, maintenance therefore it is important to establish the most effective techniques to elicit sustained behaviour change. Behavioural and psychological theories provide the theoretical framework for determinants of diet and physical activity behaviour. Although no consensus exists on the best model to use, the use of psychological behaviour change theory is generally believed to improve the outcome of behaviour change intervention ${ }^{213}$. A wide variety of behaviour change theories have been employed in weight loss interventions but the most frequently cited are the social cognitive theory (SCT), the trans theoretical model (TTM) and self-determination theory (SDT) ${ }^{214}$. 
Central to the TTM, developed by Prochaska and DiClemente ${ }^{215}$ are the six stages of change including: 1) pre-contemplation, 2) contemplation, 3) preparation, 4) action, 5) maintenance, and 6) termination. It is proposed that behaviour change follows this sequential pattern and is more likely to be successful if interventions target the needs of a specific stage, thereby increasing the likelyhood of progressing to the following stage ${ }^{215}$.

The TTM has been successfully applied in dietary and weight loss interventions in overweigh and obese subjects. For example, Dallow and Anderson 216 randomised 58 sedentary, obese women to receive a 24 -week physical activity program based on the TTM or a traditionally structured exercise program based on a personalised exercise prescription. Sustained improvements in physical activity were seen in the TTM intervention group but not the exercise prescription demonstrating that the application of behaviour change theory in the form of the TTM significantly improved activity level and maintenance. Similarly, a 14-week course of nutrition lessons, applying behaviour change processes matched to stage of change, i.e, pre-action oriented change process during the pre-contemplation and contemplation stages such as increasing knowledge and raising awareness of problem behaviour, lead to significant reduction in dietary fat intake relative to control ${ }^{217}$. However, two systematic reviews in $2011^{218}$ and $2014^{219}$ examining randomized clinical trials applying the TTM in lifestyle change interventions for treatment of overweight and obesity, concluded that the effect of the TTM was inconsistent owing to only marginal difference with control groups and a lack of quality evidence ${ }^{218,219}$.

In contrast to other theories the self-determination theory (SDT) is focused on the acquisition of motivation for the new desired behaviour. According to SDT, to achieve sustained behaviour change, an individual must internalize the value of the desired behaviour and experience self-determination. Furthermore, by improving the feeling of autonomy, competence and relatedness the adoption and self-regulation of the desired behaviour is more likely to be internalized ${ }^{220}$. The goals from which motivation stems also have intrinsic or extrinsic properties which influence behaviour maintenance ${ }^{209}$. For example, intrinsic goals such as health outcomes, personal growth or social interaction require more selfdetermined forms of regulation and are associated with greater adherence relative to extrinsic goals such as physical appearance, attractiveness or goals 
that tend to be externally regulated ${ }^{221}$. Indeed, autonomous regulation of motivation was positively associated with weight loss in the diabetes prevention program ${ }^{222}$. Similarly, obese children with intrinsic motivation showed greater adherence to physical exercise in a weight loss program compared with children with lower intrinsic motivation ${ }^{223}$.

Given that the underlying causes of obesity including genetic, environmental, psychosocial, and physiological factors are varied, and because the relative contribution of these factors is unknown, individuals are left to develop their own attributions for the cause of their weight status. Attribution theory suggests that causal attributions impact on weight related behaviours. There are three domains of causal attribution: locus of control (internal/external), stability (changeable/not changeable), and controllability (controllable/ uncontrollable) 224. Causal attributions that are perceived to be more internal, stable, and uncontrollable are associated with reduced engagement in goal-directed behaviour ${ }^{224}$. For example, overweight and obese individuals with genetic attribution of weight status, which is stable, internal and unchangeable, have been shown to be more susceptible to further weight gain ${ }^{225}$. Attributions appear to be particularly important in weight loss maintenance; individuals who attribute their obesity to physical rather than behavioural causes are also prone to setting unrealistic weight loss targets which are in turn detrimental to long-term weight loss maintenance ${ }^{226}$.

The Social cognitive theory (SCT), suggests a model whereby cognition, environment, and behaviour continually interact in a reciprocal manner to explain human behaviour. A basic premise of the SCT is the ability to analyse one's thoughts and feelings, to self-regulate, and to observe others and learn from their actions ${ }^{227}$. Further, Bandura identifies six core determinants of behaviour: 1) knowledge of health risks and benefits of alternative practices, 2) perceived self-efficacy over the desired behaviour, 3) outcome expectancy (the expected benefits and costs of the desired behaviour, 4) health goals and the necessary plans for goals to be realised, 5) perceived facilitators and social support, and 6) barriers to making changes ${ }^{228}$. 
Central to the theory of planned behaviour ${ }^{229}$, is the tenet that intention is a key determinant of behaviour. Within lifestyle interventions, implementation intention techniques are used to translate an intention towards a target behaviour into action. Implementation intentions are plans that specify what will be done and in what circumstances to achieve the desired goal. The implementation intentions are often formed from "if-then" plans that specifies an action "I will go for a swim" committing to a specific course of action when set conditions are met. "When I drive past the pool on my way home" ${ }^{230}$.

However, distinct from action planning, implementation intentions also anticipate critical situations, opportunities and potential obstacles. For example, in the context of dietary and physical activity intervention anticipation for situations to deviate from the desired behaviour. "If I see an escalator then I will take the stairs" If the waiter offers me desert, then I will ask for fruit salad ${ }^{231}$. Implementation intentions have been shown to improve goal attainment in a number of health behaviours including improved fruit and vegetable consumption ${ }^{231}$, lower saturated fat intake ${ }^{231}$ and increasing physical activity levels ${ }^{232}$.

The Health Belief Model ${ }^{233}$ is another theoretical framework frequently applied in public health interventions. It focuses on motivation to undertake a health behaviour at the individual level considering three categories: individual perceptions, modifying behaviours, and likelihood of action. Key constructs of the health belief model are perceived susceptibility, perceived severity, perceived barriers, perceived benefits and cues to action. Later models also include self-efficacy ${ }^{234}$. While there are many behaviour change models that explain, or predict behaviour, there is little consensus on which theoretical framework provides the best basis for an intervention. Some have suggested that interventions that are underpinned by a theoretical behaviour change model are more successful and changes are better maintained ${ }^{235}$. However, the evidence that theory based interventions lead to greater improvements health behaviours is not consistent ${ }^{236}$. This is possibly attributable to the wide range of available models, the lack of analysis used to inform the selection of appropriate theory and its application ${ }^{237}$. Furthermore, while many interventions are said to be grounded in theory, the application of theory is in fact minimal ${ }^{237}$. 
To address these issues, the COM-B model and the behaviour change wheel were developed by Michie and colleagues ${ }^{238}$ to provide a systematic and theory driven approach to categorise and design behaviour change interventions. The central premise of the COM-B (Capability, Opportunity, Motivation, Behaviour) model is that for behaviour to occur, individuals or populations must have the capability, opportunity and motivation to perform the desired behaviour. The COM-B model sits in the centre of the behaviour change wheel which contains intervention functions and policy categories (see Figure 3.1).

Figure 3. 1 Behaviour change wheel

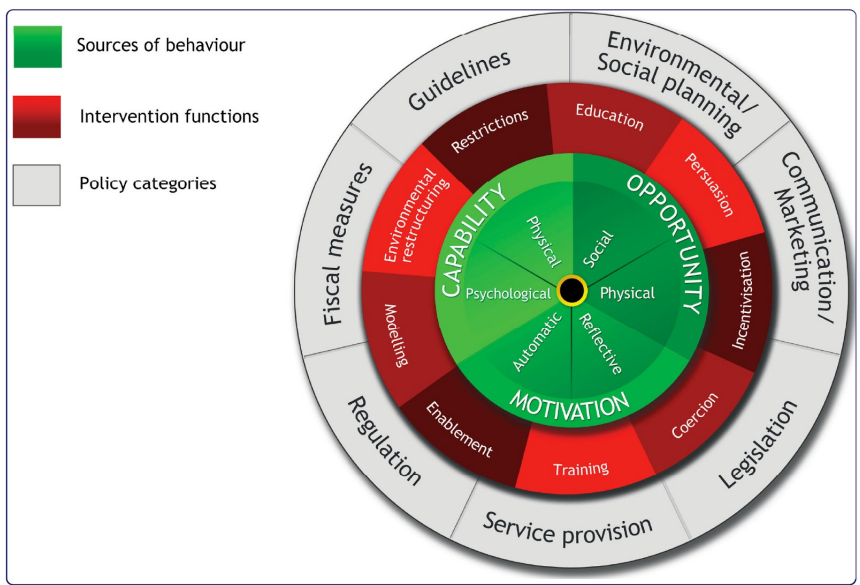

Image taken from Michie, Stralen, and West, 2011 238

The first steps of the behaviour change wheel process are to identify the specific goals of the intervention, the target behaviour, what needs to change, to what degree, in what way and in whom. The second step is to establish what changes are required in respect of capability, opportunity, and motivation in order for the desired change to take place. The next step is to define the intervention strategy by aligning the appropriate intervention function with the chosen component of the COM-B model. The outer ring of the behaviour change wheel helps to identify the policy options that are applicable to the delivery of the chosen intervention functions. The final step is to specify the intervention content in the form of behaviour change techniques to influence the desired intervention function $^{238}$.

The use of behaviour change techniques is repeatedly associated with improved outcomes in health behaviour interventions ${ }^{214,239}$. In a systematic review of 
reviews, Graves et al. ${ }^{239}$ identified goal-setting, prompting self-monitoring, providing feedback on performance, use of relapse prevention strategies, selftalk and social support as behaviour change techniques associated with greater intervention effectiveness ${ }^{239}$. In a more recent review, self-regulation of behaviour, goal setting and a person-centred delivery style supportive of autonomy were associated with greater long term behaviour change ${ }^{240}$. Similarly, in a review of mediators of sustained behaviour change, autonomous motivation, self-efficacy, and self-regulation were predictive of improved weight and physical activity ${ }^{214}$. Taken together, these behaviour change theories and techniques provide the basis for lifestyle interventions for the preventing of type 2 diabetes. However, it is not clear what the relationships are between these variables and physical activity in a population with pre-diabetes.

Behaviour change theory and techniques are also widely applied in child interventions however the efficacy of these techniques remain largely untested in paediatric populations ${ }^{34}$. In a review of effective component of interventions involving parents, specific behaviour change techniques (environmental restructuring, specific goal setting, monitoring and barrier identification) were associated with greater intervention effectiveness ${ }^{241}$.

Alternatively, in a more recent review of behaviour change techniques in childhood obesity, Martins et al ${ }^{34}$ found inconclusive evidence for the inclusion of common techniques (self-monitoring and goal setting) whereas, three techniques (provide information on the consequences of behaviour in general, provide rewards contingent on successful behaviour and facilitate social comparison) were exclusively associated with ineffective interventions. The authors proposed that the inclusion of these techniques may in fact be hampering interventions effectiveness ${ }^{34}$.

\subsection{Lifestyle interventions}

\subsubsection{Adults}

Lifestyle interventions have emerged as a viable approach to prevent or delay the onset of type 2 diabetes in high risk individuals, comparing favourably to pharmacological intervention ${ }^{20}$ and deemed financially viable ${ }^{242}$. Landmark 
studies in this area include the US Diabetes Prevention Program (DPP) ${ }^{20}$, the Finish Diabetes Prevention Study (FDPS) ${ }^{21}$ the China Da Qing Diabetes Prevention Study (CDQDPS) ${ }^{22}$, the Indian Diabetes Prevention Program (IDPP) $)^{243}$ and a Japanese diabetes prevention trial ${ }^{244}$. These randomized control trials were designed to achieve modest weight loss (5\%-7\%) through lifestyle modification to reduce the incidence of diabetes in people with impaired glucose tolerance. The interventions used group or individual counselling to reduce total dietary intake, saturated fat and simple sugars and increase dietary fibre, fruits and vegetables and physical activity. The DPP showed at 2.8 years follow up, lifestyle intervention lead to $58 \%$ reduction in incidence of type 2 diabetes compare to placebo, which was significantly better than Metformin ${ }^{245}$. Similarly, the FDPS reported 58\% reduction in diabetes incidence at 3.2 years follow up ${ }^{195}$. At 6 years, the CDQDPS showed $31 \%, 46 \%$ and $42 \%$ reductions in risk of developing diabetes, for diet, exercise and diet plus exercise respectively, compared to control ${ }^{26}$. The lifestyle modification branch of the IDPP resulted in a $28.5 \%$ reduction in relative risk of type 2 diabetes at 3 years while the Japanese diabetes prevention study reported $67.4 \%$ reduction in risk at 4 years ${ }^{244}$. Follow-up of all three large lifestyle intervention studies has shown sustained reduction in the rate of conversion to type 2 diabetes; $43 \%$ reduction in the 20 year follow up of the CDQDPS ${ }^{22}, 34 \%$ reduction at the 10 year follow up to the DPP ${ }^{246}$ and $36 \%$ reduction at 7 years in the extended follow up of the FDPS ${ }^{21}$. Remaining free of diabetes at 7 years in the FDPS was closely related to adherence to dietary physical activity goals and weight loss maintenance ${ }^{21}$. These large clinical trials provide compelling evidence for the delay or prevention of type 2 diabetes but also highlight the importance of weight loss maintenance and sustained behaviour change.

A disadvantage of the intervention programs featured above, is their intensive intervention protocol. For example, the DPP consists of 16, one-to-one sessions in the first 24 weeks, 2 voluntary exercise sessions per week, follow up visits and phone calls ${ }^{20}$. It may be difficult to translate this resource intensive strategy into usual health care practice. Structured education programs offer the potential for a more pragmatic approach that can be deliverable in a community setting or be integrated into routine care. The diabetes education and self-management for ongoing and newly diagnosed (DESMOND) program is currently available in 
primary care trusts in England and Scotland ${ }^{247}$. The DESMOND program consists of a six-hour group education program, delivered in either one day or two half days. A cluster, randomised control trial of 824 participants from 207 general practices showed that the DESMOND program resulted in greater improvements in weight loss, smoking cessation and improvements in beliefs about illness compared with control at 12 months. Small nonsignificant reductions in $\mathrm{HbAlc}$ were also found ${ }^{247}$. Unfortunately, at 3 years, improvements in biomedical and lifestyle outcomes were not sustained which may support a more ongoing form of intervention ${ }^{248}$.

Based on the philosophy of the DESMOND program, The Prediabetes Risk Education and Physical Activity Recommendation and Encouragement (PREPARE) program combined a 3-hour structured education program with pedometer use ${ }^{249}$. In a randomized control trial, overweight and obese adults with IGT were randomized to receive 3-hour structured education alone, structured education plus personalized step goals and pedometers or written information. At 12 months, the pedometer group produced a significant decrease in 2-h glucose and fasting glucose compared with the control ${ }^{249}$. Significant improvements were also maintained at 2 years ${ }^{250}$. The education group, without pedometers, produced no improvement in blood glucose control. This suggests the self-regulation strategy provided by the pedometer was a vital component of the intervention. The PREPARE intervention was subsequently developed into the Walking Away from Type 2 diabetes program ${ }^{251}$, to be delivered to individuals with a high risk of type 2 diabetes through primary care pathways in the UK. In an evaluation of 808 participants from 10 general practices, no differences between intervention and control were observed for markers of cardio-metabolic health, and while a small increase in physical activity was seen at 12 months, it was not maintained at 3 years ${ }^{251}$. Although these interventions offer a low resource program that can be delivered through primary care pathways, these findings suggest that more intensive intervention strategies are required to produce sustained behaviour change.

In an intervention tracking the progression to type 2 diabetes in high risk adults, the Let's Prevent Diabetes study ${ }^{252}$ combined the structured education program of DESMOND, the use of pedometers and daily steps goals shown to be effective by the PREPARE program, with 5\% weight loss target and dietary 
goals. Participants also received refresher sessions at 12 and 24 months and a 15-minute telephone call every 3 months. In a cluster randomised control trial of 880 participants from 44 centres, participants were randomised to receive either standard care or the structured education program. At 3-years, the reduction in incidence of type 2 diabetes was not significant, however, the intervention did lead to significant improvements in markers of cardio-metabolic health, sedentary time and step count ${ }^{252}$.

\subsubsection{Physical activity in lifestyle interventions}

In the flagship diabetes prevention studies mentioned above, physical activity targets were to engage in $>30$ minutes/day ${ }^{243,253}$ or $>150$ minutes/week ${ }^{245}$ of moderate intensity exercise, in some cases in addition to resistance exercise ${ }^{253}$. Although the promotion of physical activity was an important component of these studies, it was combined with a calorie restricted diet and a weight loss target of between 5-7\%. Consequently, the effect of physical activity on the risk of diabetes, independent of other factors, is unclear. Although not frequently studied in isolation, the CDQDPS assessed the effect of physical activity on incidence of type 2 diabetes both alone and combined with a dietary intervention ${ }^{26}$. During counselling sessions, participants were encouraged to increase their leisure time physical exercise by at least 1 unit/day, units of exercise were defined based on intensity and duration of common activities. At 6 years, the exercise intervention was associated with a $46 \%$ reduction in the incidence of type 2 diabetes, similar results were seen for the diet intervention but no additional benefit was found for combining the two. Despite the positive outcome, there was no significant increase in physical activity compared with the control group but physical activity was already greater at baseline in the exercise only group which may explain the risk reduction. Furthermore, the effect of physical activity intensity was not reported nor was it clear how much of this association was mediated by changes in body weight ${ }^{26}$. Similarly, in the FDPS total physical activity was not significantly increased in the intervention group. However, post-hoc analysis of the complete sample (intervention and control combined), showed an increase in moderate-to-vigorous leisure time physical activity was associated with a reduction in the risk of diabetes. Even light intensity activity such as walking was associated with a reduction in risk 
that improved with the duration and intensity in a dose response manner. After controlling for diet, an increase in structured, leisure time physical activity was associated with $57 \%$ reduction in risk of type 2 diabetes. This was reduced to $47 \%$ after accounting for changes in BMI suggesting that physical activity reduces the risk of type 2 diabetes partially through the reduction and maintenance of body weight but also through mechanisms independent of weight loss ${ }^{156}$. This is further supported by the DPP and the FDPS that showed individuals who met their physical activity goals but not their weight loss target had a $70 \%{ }^{195}$ and $44 \%{ }^{196}$ reduction in diabetes incidence, respectively. Weight loss is the strongest predictor of reduced diabetes incidence ${ }^{156,196}$ and although physical activity interventions alone (without calorie restriction) have modest effects on weight loss, physical activity does play an important role in preventing weight regain after initial weight loss ${ }^{24}$ as was evident in the DPP 196

There is strong evidence for a causal link between physical activity and improved insulin resistance through mechanisms independent of adiposity ${ }^{25}$. Subsequently, physical activity interventions can improve insulin resistance in those with IGT ${ }^{249,254}$. For example, the Prediabetes Risk Education and Physical Activity Recommendation and Encouragement (PREPARE) program used a 3-hour structured education program to significantly increase objectively measured ambulatory activity and self-reported MVPA. At 12 months, body weight and waist circumference were unchanged but a significant reduction in 2$\mathrm{h}$ glucose levels were found that were maintained at 2-years ${ }^{250}$.

\subsubsection{Physical activity intensity}

In the diabetes prevention trials outlined above, lifestyle interventions that combined moderate intensity activity with calorie restriction and weight loss reduce the risk of type 2 diabetes in adults with IGT ${ }^{26,243,245,253}$, though, none of these studies explored the effect of manipulating physical activity intensity in their intervention protocols. In the FDPS, despite only producing modest improvements in MVPA and no change in total physical activity, individuals with the greatest increase in MVPA saw the biggest reductions in diabetes risk. However, benefits were also seen for low intensity activity and walking ${ }^{156}$. 
Current guidelines for improvements in cardio respiratory fitness and metabolic health recommend engaging in $>150 \mathrm{~min}$ of moderate-intensity, vigorousintensity aerobic physical activity for a minimum of $20 \mathrm{~min}$ on three days each week or a combination of the two ${ }^{255,256}$. Although exercise of a greater intensity may be more beneficial than low intensity activity for metabolic health and disease endpoints ${ }^{257}$, it is unclear how this translates into intervention in adults at high risk of diabetes. Given that a majority of adults with diabetes and prediabetes are not sufficiently active ${ }^{193}$ and a "lack of time" is a commonly named barrier in this population ${ }^{258}$, shorter bouts of high intensity activity may provide an appropriate alternative. Studies of HIIT in adults at high risk of diabetes provide intriguing evidence that improvements in metabolic markers, including insulin resistance, can be achieved with short bouts of high intensity aerobic exercise. However, few studies have been conducted in a "real world" setting raising questions about external validity ${ }^{161}$. It is important to note that in all the diabetes prevention trials mentioned above, physical activity was assessed with subjective questionnaires which have limited reliability and validity relative to laboratory measures ${ }^{28}$. Specifically, questionnaires appear to be better at detecting vigorous activity than lower intensity activity and are therefore likely to underestimate associations with light and moderate activity ${ }^{30}$. Furthermore, misclassification appears to affected by BMI; Warner et al. ${ }^{29}$ found that obese participants were more likely to misclassify the intensity of physical activity and overestimated time spent in vigorous physical activity.

\subsubsection{Children}

The growing prevalence of type 2 diabetes diagnosed among children and adolescents combined with the progressive nature of the complications and comorbidities associated with overweight and obesity, provide a compelling rationale for interventions during childhood. Indeed the pre-diabetic state of IGT is fairly common in obese children with reports ranging from $15 \%$ to $25 \%{ }^{69,70}$. Similarly, the underlying cause of CVD, atherosclerosis, develops during childhood and is positively associated with BMI, ${ }^{259}$ with BMI duringchild hood, predicting CVD in adulthood ${ }^{260}$. Although not all children with IGT or IFG develop type 2 diabetes, progression to type 2 diabetes is related to the severity of obesity and weight gain while a reduction in BMI is predictive of a return to 
normal glucose tolerance ${ }^{72,73}$. The interventions reviewed above provide a comprehensive argument for lifestyle intervention for the prevention of type 2 diabetes in adults. However, clinical trials in children are lacking for the understandable reason that very long follow-ups would be required to track the progression of type 2 diabetes over a lifetime.

In 2005, a Cochrane review of interventions to prevent obesity in children concluded that most interventions that combined dietary and physical activity resulted in some improvement in behaviour but did not significantly improve BMI. The authors could not recommend a specific intervention and called for intervention design to be reconsidered and clearer reporting of the intervention process ${ }^{261}$. Since this study two Cochrane reviews have been published in this area, one on interventions for treating obesity in children ${ }^{32}$ and the other on interventions for preventing obesity in children ${ }^{262}$. The first concluded that behavioural lifestyle interventions can produce significant and clinically meaningful reduction obesity among children and adolescents ${ }^{32}$, and while the latter study concurred that child obesity programmes could have a positive effect on BMI, the large heterogeneity between studies and publication bias (the omission of small studies with negative outcomes) hampered the interpretation of the results ${ }^{262}$. In other meta analyses sedentary behaviour interventions ${ }^{263}$, family based intervention ${ }^{264}$ and combined lifestyle interventions ${ }^{31}$ have been shown to produce significant reductions in BMI, and cardio-metabolic risk factors in obese children and adolescents in the short to medium term. Although these studies are encouraging, response to weight loss interventions vary widely between participants ${ }^{31,32}$ and many that do achieve weight loss experience weight regain at later follow up ${ }^{33}$.

\subsubsection{Lifestyle behaviours associated with obese children}

Successful interventions must focus on the modifiable lifestyle components that influence obesity. Lifestyle factors such as skipping breakfast, screen time, and the consumption of sugar sweetened beverages, sweets and dairy, have been implicated in the development of obesity in children ${ }^{265-267}$. On the other hand, results from large population studies remain inconsistent ${ }^{35,36}$. Indeed, a study of 137,593 youth (10-16 years) from 34 countries found that low physical activity 
levels and television viewing were associated with obesity, but no association was found for the intake of fruit, vegetables or soft drinks or the time spent using computers. Surprisingly, a negative association was found between the frequency of the consumption of sweets (candy in the USA) and obesity ${ }^{36}$. Furthermore, the ISCOLE study of 6,025 children (9-12 years) from 12 countries, failed to find an association between dietary patterns and obesity ${ }^{35}$, in accord with population-level studies in the UK ${ }^{268,269}$. However, methodological limitations, such as the pooling of responses to a food frequency questionnaire to produce a "healthy" and "unhealthy" score ${ }^{268,269}$, precludes further interpretation of these findings. Given the counterintuitive and inconsistent associations between obesity and specific foods, defining foods as "healthy" and "unhealthy" in the context of obesity and weight gain, may not be appropriate. For example, cross-sectional and prospective studies have shown full fat milk and dairy fat to be negatively associated with obesity and weight gain ${ }^{138,270}$, Yet, full fat milk has been classed as "unhealthy", while skimmed varieties have been classified as "healthy" for assessing associations with obesity ${ }^{268,269}$. Consequently, creating a composite score may mask associations between specific foods and obesity. There is evidence that screen-based behaviours have changed across Europe over the past 15 years. Specifically, TV viewing has decreased, while computer use for gaming and non-gaming purposes has sharply increased ${ }^{271}$. Likewise, changes in dietary behaviours have been reported over the last decade, whereby the UK National Food Survey shows there has been a year-on-year shift from full fat to skimmed milk varieties and an increase in consumption of wholemeal bread ${ }^{272}$. Previous analysis of the SportLinx study show improvements in the consumption of fruit, vegetables, and salad ${ }^{273}$. In the UK, there have also been changes in the composition of foods. In 2008, 55 percent of soft drinks consumed by UK children contained no added sugar compared to 46 percent in $2004^{274}$. Despite these changes, it is unclear whether lifestyle behaviours and food choices are associated with body fat and whether these associations have changed over time. To our knowledge, no study has investigated the consistency of the associations between these lifestyle behaviours and body fat percent. 


\subsection{Summary}

This literature review has summarised the current evidence surrounding the growing prevalence of type 2 diabetes and its prevention through lifestyle interventions, with specific focus on physical activity and a view to applying interventions in children.

Type 2 diabetes has reached epidemic proportions in westernized countries and is growing rapidly in the developing world representing a major cause of global morbidity, mortality and consequently health care expenditure ${ }^{55}$. Although a genetic predisposition, age, sex, family history and ethnicity contribute to the risk of developing type 2 diabetes, the strongest risk factor is excess body weight 100. Consequently, it is widely believed to be preventable through lifestyle modification ${ }^{7}$, specifically, through weight management, improved diet and physical activity. Indeed, there is compelling evidence that lifestyle interventions can be effective to delay or prevent the onset of type 2 diabetes in adults with pre-diabetes. Weight loss significantly improves insulin resistance and risk of type 2 diabetes ${ }^{109,212}$. Physical activity improves insulin resistance independent of weight loss and contributes to weight loss maintenance ${ }^{24,25}$. However, these benefits only persist while physical activity continues and weight loss is maintained. Unfortunately, weight regain is common with many individuals returning to original behaviours following the removal of an intervention ${ }^{23}$. Engendering sustained behaviour change is a complex and difficult process requiring the application of behaviour change theory and the understanding of the underlying influences on health behaviours such as physical activity. Psychosocial factors such as self-efficacy, social support and inactivity temptation have often been shown to influence physical activity behaviours and comprise an important part of lifestyle interventions ${ }^{197}$. However, it remains unclear whether the same factors are associated with physical activity in adults with pre-diabetes; the very population at which many lifestyle interventions are targeted. Given the global nature of the diabetes epidemic, it would also be pertinent to know if the same factors are important across countries. This uncertainty is extended by the limited reliability and validity of subjective physical activity questionnaires ${ }^{28}$ and the dearth of evidence using objective measures of physical activity. Studies of the associations between objectively measured physical activity and markers of cardio-metabolic health are also 
scarce in adults with pre-diabetes. Physical activity reduces insulin resistance and the incidence of type 2 diabetes at a range of intensities ${ }^{160}$ but given that overweight and an obese adults tend to overestimate time spent in vigorous physical activity ${ }^{29}$ and questionnaires appear to be better at detecting vigorous activity than lower intensity activity ${ }^{30}$ it is likely that associations with light and moderate activity have been underestimated.

The rise in childhood obesity, IGT and early diagnosis of type 2 diabetes 9,10,38,39 would propose that for many, interventions in adulthood come too late. Indeed, the pathogenic processes of both type 2 diabetes and CVD are progressive and originate in childhood. Interventions during childhood specific to the prevention of type 2 diabetes are lacking and responses to obesity interventions are varied 31,32 . Effective obesity prevention and management interventions will need to target modifiable food and activity behaviours that influence energy balance, though population level studies are not decided on what these are ${ }^{35,36}$. Moreover, it is not known if these behaviours change over time as a consequence of evolving food systems and public choice. It is well known that behaviour change is difficult, many people are not successful and experience weight gain ${ }^{275}$. Central to successful interventions is the inclusion of a psychological behaviour modification framework and behaviour change techniques ${ }^{32}$. However, behaviour change techniques have largely been developed in adults and the process can be demanding and personal. Evidence for the effectiveness of these techniques in children is inconclusive and suggests some techniques may even be detrimental to intervention effectiveness ${ }^{34}$. 


\subsection{Objectives}

1 To quantify physical activity, sedentary time and sleep using a $24 \mathrm{~h} \cdot \mathrm{day}^{-1}$ seven-day accelerometer protocol in adults with pre-diabetes and investigate the associations between objectively measured physical activity and sedentary time with cardio-metabolic risk factors.

2 To determine associations between personal and psychosocial variables and objectively measured physical activity in adults with pre-diabetes from multiple countries and to examine inter-country differences.

3 To (i) identify key lifestyle behaviours that were associated with body fat percent and (ii) determine whether these behaviours have remained constant over a 10 -year period in children aged 10-12 years.

4 To assess the feasibility of running a lifestyle intervention to improve risk factors for type 2 diabetes in overweight and obese children using a descriptive multi-method case study approach to describe the behaviour change process. 


\section{Methods}

This chapter describes the methods of the PREVIEW and SportsLinx studies. In each experimental chapter, a more general description is provided with attention given to variables and study design relevant to the analysis conducted in that chapter.

\subsection{Study 1 and 2 PREVIEW intervention}

The Prevention of Diabetes through Lifestyle Intervention and Population Studies in Europe and around the World (PREVIEW) study, is an international, EU funded clinical trial for the prevention of type 2 diabetes (ClinicalTrials.gov, NCT01777893). The methods have been published in detail elsewhere ${ }^{276}$, this chapter provides a detailed description of the methods that are relevant to studies 1 and 2.

Study 1: Objectively Measured Physical Activity and Sedentary Time Are Associated With Cardiometabolic Risk Factors in Adults With Prediabetes: The PREVIEW study

Study 2: Personal and psychosocial correlates of physical activity in adults with pre-diabetes from 8 countries: The PREVIEW study

\subsubsection{Participants and settings}

Participants were recruited between June 2013 and February 2015 from eight study sites: University of Copenhagen (Denmark), University of Helsinki (Finland), University of Maastricht (The Netherlands), University of Nottingham (UK), University of Navarra (Spain), Medical University of Sofia (Bulgaria), University of Sydney (Australia), and University of Auckland (New Zealand). Recruitment methods varied by centre and included: newspaper advertisements, newsletters, radio and television advertisements/interviews, and direct contact with health care providers. After making contact, individuals were called for a pre-screening telephone interview. In the interview, inclusion and exclusion criteria were queried, including the Finnish Diabetes Risk Score ${ }^{277}$ which included questions on age, BMI, waist circumference, use of blood pressure 
medication, history of high blood glucose, physical activity levels, and consumption of fruit and vegetables ${ }^{277}$. The inclusion criteria were: age 25-70 years, overweight or obese (BMI $\left.>25 \mathrm{~kg} / \mathrm{m}^{2}\right)$ and pre-diabetes confirmed by an oral glucose tolerance test (OGTT) using the American Diabetes Association (ADA) and International Diabetes Foundation (IDF) criteria: Impaired Fasting Glucose (IFG): Fasting venous plasma glucose concentration 5.6 - $6.9 \mathrm{mmol} / 1$ or Impaired Glucose Tolerance (IGT): Venous plasma glucose concentration of 7.8 - $11.0 \mathrm{mmol} / \mathrm{l}$ at $2 \mathrm{~h}$ after oral administration of $75 \mathrm{~g}$ glucose (oral glucose tolerance test, OGTT), with fasting plasma glucose less than $7.0 \mathrm{mmol} / \mathrm{l}$. Exclusion criteria were type 2 diabetes, or any illness or medication with the potential to affect the ability to follow the protocol or the main study outcomes. Eligible participants were invited to attend an information meeting where the study was explained in full and written and oral information was provided and participants were given the chance to ask questions. Interested participants signed informed consent and a laboratory screening was scheduled. Signed informed consent was required prior to attending the laboratory screening. The laboratory screening involved measurements of weight, height, resting blood pressure, electrocardiography (in those aged 55 years or more), and an OGTT. A fasting blood sample was collected from the ante-cubital vein for later assessment of full inclusion and exclusion criteria, whilst glucose concentration was immediately analysed at each study site $\left(\mathrm{HemoCue}^{\mathrm{TM}}\right.$, Angelholm, Sweden; Reflotron $^{\mathrm{TM}}$, Roche diagnostics, Switzerland; or EML105 Radiometer, Copenhagen).

\subsubsection{Instruments and Procedures}

Anthropometrics included body mass, stature, sitting stature, waist, hip and thigh circumferences, body composition and fat distribution.

Body mass was measured in a fasted state with an empty bladder wearing underwear or other light clothing. Two measurements were taken to the nearest $0.1 \mathrm{~kg}$. This process was repeated and the average of each of the two weights was used in the analysis.

Stature was measured to the nearest $0.5 \mathrm{~cm}$ with a wall-mounted stadiometer. Participants were required to remove shoes and keep their heels, buttocks and 
upper part of the back in contact with the stadiometer. The measurement was repeated and the average of the two heights used in the analysis.

Sitting stature was measured with a wall-mounted stadiometer measuring to the nearest $0.5 \mathrm{~cm}$. Participants were required to sit on a flat, wooden shelf, placed on a seat and have his/her buttocks and upper part of the back remaining in contact with the stadiometer. The measurement was repeated and the average of two sitting height measurements used in the analyses.

Waist circumference was taken midway between the lower rib and iliac crest, at the end of expiration.

Hip circumference was measured as the largest circumference in the area around the buttocks.

Thigh circumference was taken on the right side of the body at the level of the mid-point on the lateral surface of the thigh, midway between trochanterion and tibiale laterale.

Waist, hip and thigh circumferences were measured to the nearest $0.5 \mathrm{~cm}$ with the same tape while the participant was standing. Two measurements were taken and the average used.

Systolic and diastolic blood pressure were measured using a validated automatic device on the right arm after 5-10 min rest in a seated position. Measurements were taken three times with a 1 min rest between, and the reading was recorded to the nearest $1 \mathrm{mmHg}$. A mean value of these three readings was used.

\section{Blood samples}

Participants arrived in a fasted state (10-12 hour), only a small glass of water was permitted $(1 \mathrm{dL})$. Blood samples were taken from the vein in the antecubital fossa using either an evacuated tube system or with cannula while the participants were in semi-recumbent position. Venosafe blood sampling tubes were used (VenoSafe 5/3 ml fluoride-citrate tube [VF-053SFC], VenoSafe 10/8 $\mathrm{ml}$ Gel serum tube [VF-108SAS] and VenoSafe 10/9 ml EDTA tube [VF109SDK]) employing standardised procedure and blood sample tubes across the 8 study sites. After a total of 6 fasting blood sample tubes were taken, each participant performed a 2-hour Oral Glucose Tolerance Test (OGTT); an oral load of $75 \mathrm{~g}$ glucose was dissolved in $300 \mathrm{ml}$ water and consumed within a 5min period. Participants remained semi-recumbent and resting throughout the 
procedure. Blood samples were drawn for glucose assessment at 0, 30, 60, 90 and 120 and for insulin assessment at 0,30, and 120 minutes after drinking the liquid glucose solution. Glucose from 0 and 120 minute samples was analysed immediately with HemoCue to test for the presence of type 2 diabetes. All other glucose values were analysed centrally in Finland. Fasting insulin and glucose values and glucose at 120 minutes were available for analysis and used throughout this thesis.

Immediately after being drawn, all blood tubes were mixed by inverting the tubes. Fluoride-citrate tubes were mixed 15 times to help dissolve granules and prevent haemolysis while serum and EDTA tubes were mixed 5 times to dissolve anticoagulants and clotting activators, respectively. All tubes were stored in a vertical position until centrifuged at $1500 \mathrm{G}$ for 10 minutes except for an EDTA-plasma tube which was frozen as whole blood for analysis of HbA1c. Serum and plasma was then pipetted into aliquots and stored at $-80{ }^{\circ} \mathrm{C}$. All blood samples were analysed centrally at the National Institute for Health and Welfare, Helsinki, Finland. Plasma glucose was analysed via the enzymatic hexokinase method, insulin and C-peptide were measured by chemiluminescent micro particle immune assay. Serum levels of total cholesterol, HDL cholesterol, triglycerides and HbAlc were determined enzymatically, LDL was calculated using the Friedewald formula ${ }^{278}$. High-sensitive CRP was analysed by immunoturbidimetric assay. All analyses were conducted using Architect ci8200 analyser (Abbott, Chicago, IL). Insulin resistance was calculated using the homeostasis model assessment for insulin resistance, HOMA-IR using the equation: HOMA-IR $=$ Fasting insulin $\left(\mathrm{mU} \cdot \mathrm{l}^{-1}\right) \times$ Fasting glucose $\left(\mathrm{mmol} \cdot \mathrm{l}^{-1}\right) /$ 22.5. HOMA-IR has been validated against the gold standard hyperinsulinemiceuglycemic clamp technique ${ }^{279}$. Total adiposity was assessed by dual energy Xray absorptiometry (DEXA) in four (Copenhagen, Nottingham, Sydney and Auckland), bioelectrical impedance in 3 (Helsinki, Sofia, and Navarra) and with Bodpod (Maastricht.) in one site. Self-administered questionnaires assessed general and socio-economic variables, including ethnicity, educational status, household income. 


\section{Physical activity}

Participants wore an ActiSleep+ (ActiGraph LLC, Pensacola, FL) accelerometer attached to an elastic waist belt, worn over the right mid-axillary line. The ActiGraph was worn $24 \mathrm{~h} \cdot \mathrm{day}^{-1}$ for seven consecutive days, removing only for water-based activities and contact sports. Data were collected at a $100 \mathrm{~Hz}$ sampling rate, downloaded in 1-second epochs and aggregated to 60-second epochs 280,281 . Sleep time was determined using a fully automated algorithm developed for use in 24-h waist worn accelerometer protocols. The algorithm produces estimates of a nocturnal sleep period that are compared with an expert visual inspection of accelerometer trace ${ }^{282}$. After the removal of nocturnal sleep episodes participants providing at least 4 days, including at least 1 weekend day, of valid data ( $\geq 10 \mathrm{~h} \cdot$ day $^{-1}$ of waking wear time) were included in the analysis ${ }^{283}$. Non-wear was classified as 60 minutes of consecutive zeros with the allowance of interruptions up to 2 minutes as has been used in large population studies ${ }^{280}$ and shown to minimise the misclassification of sedentary time as non-wear while also retaining data ${ }^{284}$. Mean activity count during valid wear time (counts $\cdot$ minute $^{-1}$; CPM) has been shown to correlate well with total activity energy expenditure measured by the doubly labelled water technique ${ }^{27}$ and was used as an indicator of total physical activity volume. Troiano cut-points were used to determine time (minutes $\cdot \mathrm{day}^{-1}$ ) spent at different intensity categories (Sedentary $<100$, moderate $<2,020$ and vigorous $<5,999$ counts $\cdot$ minute $^{-1}$ ). Moderate and vigorous activity were summed to obtain moderate to vigorous physical activity (MVPA) ${ }^{280}$.

\section{Questionnaires}

The PREVIEW intervention is supported by psychological determinants of health behaviour change. For assessment of potential influencing of these factors validated questionnaires on social cognitive determinants of behaviour, sociodemographic and socio-economic components were used. Questionnaires were administered at baseline before participants were randomized to their intervention groups or received any dietary or physical activity guidance. All questionnaires were prepared in English and translated into respective local languages using authorized translators. A second authorized translator then translated the versions in reverse to ensure the meaning had not changed. This 
process was repeated until a final version of sufficient quality was obtained for each country where English was not the official language.

Intention. Participants' intention to "eat as healthy as possible", "exercise regularly", and "lose weight" in the next two weeks were assessed using items for Intention adapted from Renner and Schwarzer et al. ${ }^{285}$. Participants rated the three items on a Likert scale from 1 (do not intend at all) to 7 (strongly intend).

Self-efficacy. Self-efficacy for physical activity was measured using 5 items from the health specific self-efficacy scale ${ }^{286}$. Participants rated their selfefficacy on a scale from 1 (very uncertain) to 4 (very certain), with the mean value calculated. The scale had high internal consistency (Cronbach's alpha of $0.90)$.

Habit strength. Participants' habit strength of physical inactivity was measured using a questionnaire based on Wood et al ${ }^{287}$. Scored using a 7-point Likert scale, items included the frequency and stability (context stability, time stability, and location stability) of participant physical inactivity (sitting in front of a TV or computer; using an inactive form of commuting) on a typical day in the past week. For example, the frequency of physical inactivity was assessed on a scale from 1 (never/almost never) to 7 (very often/almost every day). Context stability regarding the time, location and/or the purpose of physical inactivity was reported on a scale from 1 (never/almost never) to 7 (almost always at the same time/location/purpose) for the time, location and purpose stability. To estimate inactivity habit strength, the product of the frequency and stability scores was calculated, with the derived habit strength scores ranging from 1 to 343 , with higher scores reflecting frequent performance in stable circumstances.

Goal adjustment. The self-regulation of goal adjustment scale is a 10-item instrument that encompasses goal disengagement and goal reengagement subscales ${ }^{288}$. The goal disengagement scale asked participants about the ease with which they were able to reduce effort (two items) and relinquish commitment towards unattainable goals (two items). To measure goal reengagement, participants rated the extent to which they generally reengage in 
new goals if they face constraints on goal pursuits (six items). Response options ranged from 1 (strongly disagree) to 5 (strongly agree) and mean scores were computed. Both goal disengagement and goal reengagement had fair-to-high internal consistency (Cronbach's alpha of 0.70 and 0.88 , respectively).

Causal attribution. In accordance with attribution theory ${ }^{289}$, participants were asked to rate the cause of their physical activity habits on six, 7-point, dimensional scales assessing: internality $(1=$ totally about me $)$ - externality $(7=$ totally about other people or circumstances); stability ( $1=$ never changes at all $)$ - instability ( 7 = changes constantly); uncontrollability of others $(1=$ people have no control over at all) - controllability of others ( $1=$ people have total control over $)$; and uncontrollability of self $(1=$ I have no control over at all $)-$ controllability of self $(7=$ I have total control over $)$. Participants were also asked how confident they were that one year later they would achieve their personal target weight with response options ranging from 1 (no chance at all) to 7 (completely certain).

Temptations. To measure physical inactivity temptations, the subscale competing demands from the Temptation to not Exercise Scale ${ }^{290}$ was used (six items), supplemented by the item "How tempted are you not to exercise and be sedentary while being on a business trip?". Temptations were measured on a scale from 1 (not at all tempted to not exercise/be sedentary) to 5 (extremely tempted to not exercise/be sedentary). A mean value was computed ranging from 1 to 5. There was high internal consistency between items (Cronbach's alpha $=0.88)$.

Social support. The sub-scale Social Support for Exercise Behaviour ${ }^{291}$ was used to assess participants' social support from family and friends over the past 2 months. Participants were given different statements about families' and friends' reactions to the participant's intention to change exercise behaviour, assessing social support with 10 items on a scale from 1 (none) to 5 (very often). In case the question did not apply to the participant, an additional response option was given (does not apply). Mean values were computed regarding encouragement and discouragement for being physically active separately for 
family and friends with high internal consistency (Cronbach's alpha of 0.85 and 0.90 , respectively).

Availability and accessibility. Recommended physical activity availability and accessibility questions are based on the subscale Environment of the Physical activity Instrument (IPAI) ${ }^{292}$ using 5 items. Participants were asked whether they have a place or time to be physically active and exercise. Response options ranged from 1 (strongly agree) to 4 (strongly disagree). Mean values were computed ranging from 1 to 4 , with low scores reflecting less place and time and high scores reflecting plenty of place and time.

\subsubsection{Design}

The PREVIEW study is a 3-year randomised clinical trial with the aim to determine the effects of two diets and two physical activity programmes on the prevention of type 2 diabetes in overweight, pre-diabetic adults, following a weight loss period. The PREVIEW study is currently still running and due to conclude in June 2018. Details of the analysis of baseline data, included in this thesis are provided in each experimental chapter. This thesis conducted crosssectional analysis baseline data therefore details of the intervention methods are irrelevant and have been excluded.

\subsection{Study 4: The effectiveness of a child weight management intervention; a mixed methods investigation of child and parent's perceptions of the behaviour change process}

The child arm of the PREVIEW intervention is an exploratory branch of the project being conducted in Maastricht, Navarra and Swansea. The intervention is based on the same behaviour change program and experimental diet but is delivered over two years with less invasive and intensive outcome measures, deemed more suitable for children. In the 3 intervention sites delivering the child intervention, the age of included children ranged from 10-18 years. However, in Swansea the decision was taken to focus recruitment efforts at children aged 1012. This decision was made to consolidate resources that are needed to deliver the intervention to a wide age range and to target recruitment at an age range for which we had already collected obesity prevalence data in Swansea. 
In Swansea, the study was approved by institutional research ethics committee National Institute for Social Care and Health Research Ethics Committee (14/WA/1188) and the NHS R\&D Health Board (Ref:160086).

\subsubsection{Participants/settings}

In June 2015 children, 9 children, ages of 10-12 were recruited from Swansea and the surrounding area (UK), through radio advertisements, newspaper articles, local schools and GP referral. Participants were overweight or obese according to the International Obesity Task Force criteria (IOTF) ${ }^{293}$ and insulin resistant following a screening test (HOMA-IR $>2$ ).

\subsubsection{Instruments and Procedures}

The intervention was delivered in 2 phases. Phase 1 was an eight-week low calorie diet phase (LCD) during which children were given a conventional weight-reduction diet, without a specific aim for absolute weight loss. Participants were required to maintain a stable weight while they were growing in height thereby reducing their BMI. Each participant received a personalized sample menu based on their caloric requirements accounting for sex, age, weight and physical activity level. Basal metabolic rate (BMR) was calculated using the Word Health Organization (WHO) formula: Girls BMR = $(12.2 \times$ weight $)+746$ Boys $\mathrm{BMR}=(17.5 \times$ weight $)+651^{294}$. The diet excluded energy-dense, nutrient-poor food and encouraged a high fiber, fruit, and vegetable intake, timely, regular meals and avoidance of "grazing" during the day. During this 8-week period, participants attended six group meetings on weeks $0,2,4,6$ and 8 in which dietary and behavioural instruction was delivered. On successful completion of the LCD phase, participants started the randomized intervention phase. Participants were randomized to one of the dietary interventions, $\mathrm{HP}=$ High protein $(25 \%$ of energy), moderate carbohydrate (45\% of energy), starchy food items with low Glycemic Index (GI), or MP = Moderate protein (15 E\%), high carbohydrate (55\% of energy), starchy food items with moderate GI. Diets were consumed ad libitum with respect to energy intake but examples of daily eating plans were provided to help participants achieve the required ratios of protein and carbohydrate using the 
plate model to visually demonstrate these ratios with commonly consumed foods. Instructions were given during group meetings about controlling the portion size of food types to achieve the prescribed diet using self-monitoring and regular meal patterns. Written materials were provided including a cook book specifically designed for PREVIEW. The cook book contained recipes designed to meet the requirements of their specific diet group (i.e. HP/LGI recipes or MP/MGI recipes). Moreover, Practical sessions were held to prepare meals and healthy lunch boxes that complied with dietary guidelines; one staff member was a trained chef and gave advice on preparing the meals designed for this study.

During group visits, an exercise and nutrition counsellor assisted participants in planning their personal physical activity program to achieve physical activity guidelines of $\geq 60$ minutes of moderate to vigorous activity per day ${ }^{295}$. Physical activity was, in general, unsupervised but children were taught how to identify opportunities to be active, problem-solving techniques, how to overcome urges to refrain from planned physical activities, stretching, home-based muscleconditioning exercises and how to assess intensity. Participants also received written material to help plan and evaluate their own physical activity (Appendix $4)$.

\subsubsection{Behaviour modification framework}

The protocol for the behaviour change intervention was designed by Stuttgart University for the PREVIEW intervention called PREMIT ${ }^{296}$. PREMIT is based on a trans-theoretical approach and combines valid behaviour modification theories, models and approaches Including the Social Cognitive Theory, the Self-Determination, Habit Theory, and Goal Adjustment Theory. A trained counsellor and an exercise and nutrition counsellor delivered the behaviour modification component of the intervention. Each meeting started with a discussion with participants about their progress and feedback and encouragement was provided, a recap of the previous meeting was given followed by an outline of the content and the aims of the session to come. Group meetings often incorporated games or activities (preparing food, relay race, stretching and warmups, word games) so that children could make their own 
choices and interact with their peers and gain competency in activities related to food choices and physical activity.

Action planning and goal setting were used throughout the study; children defined goals according to the SMART principles:

- Specific

- Measurable

- Attainable

- Relevant

- Time bound

Participants were encouraged to set behavioural goals as opposed to outcome goals, these goals were discussed frequently during group meetings when children received feedback, adjusted and progressed their goals. Action planning exercises were undertaken in which children outlined their desired goal and a detailed plan (what, where and when) they intend to execute them. The plan included "if-then-statements"; the "if" statement provides a situation that acts as a cue to trigger a behavioural response. The "then" specifies what will be done. The children signed a behaviour change contract stating their dietary and physical activity goals and outlining the steps needed to achieve them. Mental contrasting and implementation intention techniques were used to visualize desired outcomes and anticipate potential barriers and find solutions before they arose ${ }^{297}$. Decision prompts were discussed with the children to develop visual or situational reminders to help stick to the recommended behaviour or avoid temptation. During the action phase, self-monitoring of behaviour was introduced. Participants were encouraged to complete physical activity and dietary logs. The use of apps, step counters, and heart rate monitoring was later introduced. A system of "plan-perform-evaluate" was initiated whereby children would plan their dietary and physical activities via their SMART goals and action planners, they would perform the planned activities over the coming weeks, evaluate their performance by monitoring their behaviour, and finally, reflect on their progress and adjust their goals accordingly. From the perspective of Self-determination theory ${ }^{209}$, competency (i.e. the need to feel a sense of mastery and the ability to perform the desired behaviour) is essential to initiate and maintain behaviour change. During the study, competency was developed 
by teaching and developing skills such as cooking, making food choices, reading food labels and performing exercises stretches and warm ups. Self-efficacy, defined by the social cognitive theory as an individual's confidence in being able to carry out a behaviour, has also been shown to be a critical factor in successful behaviour change ${ }^{197,298}$. Focusing on past success and providing feedback on successful completing of goals and target behaviours promoted self-efficacy. During group meetings, autonomy was encouraged by allowing participants the choice to self-determine their behaviour, participants were encouraged to come up with their own exercise plans, dietary changes and set individual goals. Furthermore, they were provided with a rationale and reason for adopting the desired behaviour as opposed to prescribing behaviours or giving ultimatums, and self-monitoring was promoted to prevent reliance on external regulation ${ }^{299}$. Social support, in accordance with the self-determination theory, relatedness, a sense of belonging with others, is essential for autonomous motivation and behaviour maintenance ${ }^{299}$. During the group meetings, open discussion to share ideas and give feedback was designed to allow children to relate to their peers. Moreover, a buddy system was introduced to monitor each other's goals and provide support between meetings.

\section{Preparation phase}

The main objective during the preparation phase was to convince participants that poor lifestyle behaviours increased their risk of suffering from a severe disease and foster the outcome expectancy that if they change their behaviour they can prevent the onset of type 2 diabetes (or reduce their risk) by reducing their BMI and adhering to this lifestyle in the long term. During the group visits, instructors motivated participants to start the recommended diet and physical activity, focusing on participants' behavioural goals, positive outcome expectancy of adopting the preferred behaviours and the participants perceived ability to follow the recommended behaviours.

\section{Action phase}

During the action phase, the main aim was to help participants stick autonomously to the recommended behaviour. Focus was on self-regulation, monitoring behaviour, resisting temptations and adjusting behavioural goals in an appropriate manner.

\section{Maintenance phase}


After 6 months, participants reached the maintenance phase. The main aim of this stage was to prevent relapses. Counsellors helped participants to cope with difficult situations (coping self-efficacy), overcome barriers and return to the desired behaviour if a relapse had occurred.

A matrix of the objectives, tasks and tools can and the order they were delivered in can be found in the Appendix 5.

\subsubsection{Outcome measures}

Outcome measures were taken at clinical investigation days (CIDs) in week, 0, 8, 26, 52, 78, and 104. Participants attended CIDs, which were held in the mornings, following an overnight fast of between 10-12 hours. Fasting blood samples are drawn from the vein in ante-cubital fossa when the patient is in a sitting, semi recumbent or lying position. This was performed by a trained phlebotomist at Singleton Hospital. All samples were processed at the Diabetes Research Unit laboratories at Swansea University using the protocol outlined in the adult study. Fasting glucose levels were analysed by glucose oxidase method using the Raydox Datona Plus. HbA1c\% was determined from whole blood by high performance liquid chromatography using D-10 Haemoglobin Analyser (Bio-Rad laboratories, Hemel Hempstead, UK). Insulin and C-peptide were measured by immunoassay using an Invitron insulin assay kit (IV2-001/101; and C-Peptide assay kit (IV2-004/104; Invitron, Monmouth, UK). Insulin resistance was assessed through the Homeostasis model of insulin resistance (HOMA-IR) from fasting insulin and glucose measures, using the following equation: fasting glucose $(\mathrm{mmol} / \mathrm{L}) \times$ fasting insulin $(\mathrm{mU} / \mathrm{L}) / 22.5^{300}$.

Body mass was measured in a fasted state, with an empty bladder and wearing light clothing to the nearest $0.1 \mathrm{~kg}$ using medical weight scale (Seca-770 1321004). Stature was measured to the nearest $0.1 \mathrm{~cm}$ using calibrated stadiometer (Holtain limited). Participants were asked to remove their shoes and stood with their heels, buttocks and upper part of the back in contact with a wallmounted stadiometer.

Waist, hip and thigh circumferences were measured to the nearest $0.5 \mathrm{~cm}$ with the same tape, with the participant standing. Waist measurements were taken midway between the lower rib and iliac crest, at the end of expiration. Hip 
circumference was measured as the largest circumference in the area around the buttocks. Mid-thigh circumference was measured on the right side of the body, at the level of the mid-point on the lateral surface of the thigh, midway between the trochanter on and tibia lateral. All anthropometric measurements were taken twice and the average of the two measurements were used in the analysis.

Systolic and diastolic blood pressure (BP) were measured using a validated automatic device (Omron M6 comfort HEM-7221-E8) on the right arm after 510 min rest in a resting position. The measurement is taken three times with a 1 min rest between, and the reading is recorded to the nearest $1 \mathrm{mmHg}$, the mean value of the tree readings was used.

Participants wore an ActiSleep+ (ActiGraph LLC, Pensacola, FL), accelerometer attached to an elastic waist belt worn over the right mid-axillary line recording activity counts at $100 \mathrm{~Hz}$. The accelerometer was worn 24 hours/day for 7 full days, including 2 weekend days. Sleep time was determined using a fullyautomated algorithm developed for use in 24-hour waist-worn accelerometer protocols ${ }^{282}$. The algorithm produces estimates of nocturnal sleep period that are compared to an expert visual inspection of accelerometer trace. After the removal of nocturnal sleep episodes, participants were included in the analyses if they wore the monitor for $\geq 10$ hours on $\geq 4$ days including 1 weekend day. Nonwear periods were defined as any sequence of at least 20 consecutive minutes of 0 activity counts ${ }^{301} 302$. Evenson cut points ${ }^{303}$ were used to determine time (min·day-1) spent at different intensity categories, moderate and vigorous activity were summed to obtain moderate to vigorous physical activity (MVPA). Each participant was asked to complete a 4-day food diary. The inclusion of 3 weekdays and 1 weekend day were specified to account for the variation in food intake at weekends ${ }^{304}$. Participants were encouraged to maintain their usual eating pattern during the assessment and use a small scale to weigh their foods. All food diaries were analysed using Nutritics software (version 1.8, Nutritics Ltd., Co. Dublin, Ireland) and converted to dietary intakes and food groups using nutrient composition data tables (2015 Composition of foods integrated dataset [CoFID] McCance and Widdowson's $7^{\text {th }}$ ed). If the food item was not included in the database, information from the manufacturer was sought or, alternatively, the most similar food item was substituted. Each participant's intake of energy, 
protein, fat, fibre, total carbohydrates, total sugars, free sugars and GI were calculated using the food diaries at each time point.

\section{Dietary intake}

Each subject was asked to complete a 4-day food diary. The inclusion of three weekdays and one weekend day was specified to account for the variation in food intake at weekends ${ }^{304}$. Participants were encouraged to maintain their usual eating pattern during the assessment. All food diaries were analysed using Nutritics software (version 1.8, Nutritics Ltd., Co. Dublin, Ireland) and converted to dietary intakes and food groups using nutrient composition data tables (2015 Composition of foods integrated dataset [CoFID] McCance and Widdowson's $7^{\text {th }} \mathrm{ed}$ ). If the food item was not included in the database, information from the manufacturer was sought or, alternatively, the most similar food item was substituted. Each participant's intake of energy, protein, fat, fibre, total carbohydrates, total sugars, free sugars and GI were calculated using the food diaries at each time point.

\subsubsection{Design}

The study was a two-year (104 week) intervention consisting of two phases, namely an 8-week weight reduction phase (participants were not required to lose weight but maintain weight while growing thereby reducing their BMI), followed by the 148-week randomized weight-maintenance phase in which participants were randomized to one of two diet groups. A total of 15 group meetings and 6 CIDs were scheduled over the two years. Group meetings lasted roughly 1 hour, if a participant was unable to attend the meetings an individual meeting was arranged to allow that participant to catch up. The frequency of meetings and supervision gradually declined towards the end of the study in a "fading" design, see figure below. 
Figure 4. 1 Program design - Fading intervention

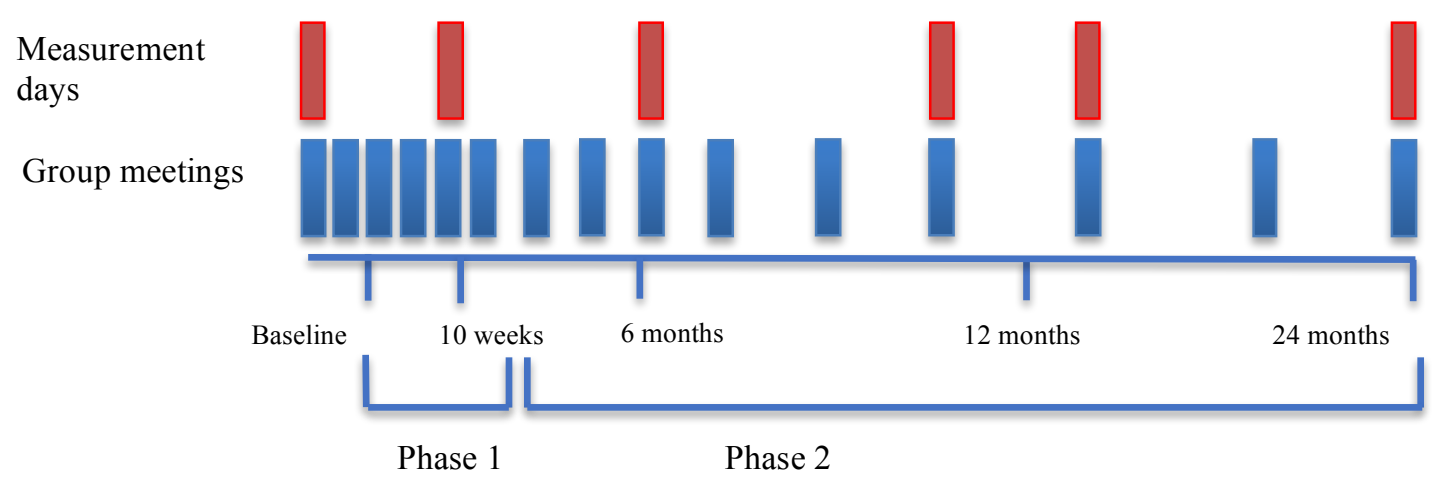

\subsection{Study 3: Lifestyle behaviours associated with body fat percent in 9-11- year-old children: The SportsLinx study}

Sportslinx is a serial, cross-sectional, health, nutrition and wellbeing program, conducted in primary schools within the Liverpool Local Education Authority (UK) by Liverpool John Moors University in conjunction with Liverpool City Council and has been running since $1996^{305}$. The study was approved by Liverpool John Moores University ethics committee (09/H1002/57).

\subsubsection{Participants and Settings}

Each year, all year 5 pupils (9-10 years) from primary schools within the Liverpool Local Education Authority (UK) are invited to take part. On average, 105 primary schools took part every year, representing $90 \%$ of all primary schools in the area. Written informed consent and assent was obtained from parents and children, respectively.

\subsubsection{Instruments and Procedures}

There were two distinct parts to the SporstLinx study; the completion of a lifestyle questionnaire (the SportsLinx Lifestyle Survey) and attendance at a "fitness fun day" at which fitness tests and anthropometric measures were taken. The SportsLinx Lifestyle Survey contains questions on sport participation, sedentary behaviours, diet, nutrition, and sleep. Participants specified, on a 5point Likert scale, how much time during week and weekend days they typically spent watching TV/DVDs/videos, playing video games, using the internet, and participating in sport. Given that spending above 2 hours in sedentary pursuits, such as TV viewing, has shown to be associated with unfavourable body composition and cardio-metabolic outcomes in children ${ }^{306}$, data were 
categorized into dichotomous levels for each variable to reflect participation in each sedentary activity for above or below 2 hour per day. The food intake questionnaire was designed to measure eating habits at the population level based on the recall of foods consumed on the previous day. A series of 'yes/no' questions indicated which foods were consumed from a list of 44 items. The reliability and validity of the food intake questionnaire has been demonstrated with children of a similar age ${ }^{307,308}$. The questionnaire was completed in the classroom supervised by a teacher and the use of online versions was encouraged.

Participants had their body mass measured to the nearest $0.1 \mathrm{~kg}$ using calibrated analogue scales (model 873, Seca, Birmingham, UK). Stature was measured to the nearest $0.1 \mathrm{~cm}$ using a Leicester Height Measure (Seca, Birmingham, UK). Body Mass Index was used to calculate body mass index standardised BMI scores (BMI z-scores), using the International Obesity Task Force values ${ }^{293}$. Overweight and obesity were defined by using the International Obesity Task Force criteria ${ }^{293}$. Socioeconomic status (SES) was calculated using the 2007 Indices of Multiple Deprivation (IMD) ${ }^{309}$. This incorporates seven domains of deprivation and is assigned to each participant based on their home postcode. Body Mass Index was used to calculate body mass index standardised BMI scores (BMI z-scores), using the International Obesity Task Force values ${ }^{293}$. Overweight and obesity were defined by using the International Obesity Task Force criteria $^{293}$.

Body fat percent. Skinfold thicknesses were measured by the same ISAK qualified anthropometrist using standardised procedures ${ }^{310}$. Skinfolds were measured at the triceps, halfway between the acromial process and the olecranon process of the left arm and subscapular at a $45^{\circ}$ angle just below the left scapula 311 . The Slaughter equations ${ }^{312}$ were used to estimate body fat percent from the skinfold thicknesses.

\section{Aerobic fitness}

The 20 metre multi-stage fitness test (MSFT) which has previously been validated in this age group ${ }^{313}$, was used to estimate the children's aerobic 
fitness. The number of shuttles completed by each participant was used to estimate aerobic fitness.

Socioeconomic status (SES) was calculated using the 2007 Indices of Multiple Deprivation ${ }^{309}$. Indices of Multiple Deprivation (IMD) incorporate seven domains of deprivation and is assigned to each participant based on their home postcode. 


\subsection{Thesis Map}

\begin{tabular}{lll}
\hline Study & Aims & Findings
\end{tabular}

1.

Objectively measured physical activity and sedentary time are associated with cardio-metabolic risk factors in adults with prediabetes: The PREVIEW study
To measure the relationship

between cardio-metabolic risk

factors and objectively measured physical activity and sedentary time.

$\mathrm{H}_{0}$ : The magnitude of the association between physical activity and cardio metabolic risk factors is equal to zero

2.

Correlates of physical activity in adults with pre-diabetes from 8 countries: the PREVIEW study

3

Lifestyle behaviours associated

with body fat percent in 9-11-year-

old children, a population-based,

cross-sectional study: The

SportsLinx project

4.

The effectiveness of a child weight management intervention; a mixed methods investigation of child and parent's perceptions of the behaviour change process 


\section{Study 1: Objectively Measured Physical Activity and Sedentary Time are Associated with Cardio-metabolic Risk Factors in Adults with Prediabetes: The PREVIEW Study ${ }^{314}$}

\subsection{Introduction}

The global prevalence of diabetes among adults has almost quadrupled since 1980 to 422 million cases in 2014 and is expected to continue to grow ${ }^{58}$. This dramatic rise in prevalence is largely due to the increase in type 2 diabetes which accounts for the majority of all diagnosed cases in adults ${ }^{58}$. Changes in lifestyle factors such as sedentary behaviour, insufficient physical activity, dietary choices and excess weight are important contributors in the development of type 2 diabetes ${ }^{5}$. Physical activity plays an important role, independent of weight, in the prevention of type 2 diabetes, through its effect on insulin resistance ${ }^{6}$. Physical activity leads to the translocation of GLUT-4 transporters to the plasma membrane increasing glucose uptake into skeletal muscle ${ }^{150}$. Sedentary time has also been associated with insulin resistance and fasting triglyceride levels, independent of physical activity and obesity ${ }^{148}$. Sedentary time is thought to affect carbohydrate metabolism by decreasing muscle GLUT-4 concentrations while also reducing lipoprotein lipase (LPL) activity and triglycerides clearance 166,167

Even light activity is associated with cardio-metabolic health and together with sedentary time, occupies a majority of waking hours ${ }^{188}$. Light-intensity activity substantially contributes to overall daily energy expenditure and may also mean spending less time in sedentary behaviours. Many studies reporting associations between physical activity, sedentary time and cardio-metabolic risk factors are limited by the self-reported measures of physical activity and sedentary time 177,178 which are susceptible to reporting and recall bias. In the limited number that have used objective measures of physical activity, investigations of international samples measured concurrently with the same protocol are lacking, little consistency exists with regards to the devices, wear time criteria, intensity cut points or epoch lengths used, thereby limiting the ability to make comparisons between these studies. Furthermore, despite those with pre-diabetes being at the highest risk of developing type 2 diabetes and representing the population at which many lifestyle interventions are targeted, few studies have 
described the relationship between physical activity and insulin resistance in this population.

Typically, epidemiological research assessing the effect of physical activity and sedentary time on cardio-metabolic risk factors has been conducted in the general population ${ }^{188-190}$. The few studies conducted in high risk populations have included participants based on a risk score questionnaire ${ }^{148}$, family history of type 2 diabetes ${ }^{177,191}$ or participants diagnosed with diabetes and pre-diabetes have been combined ${ }^{192}$. Consequently, physical characteristic and metabolic parameters of study participants varied substantially. As a result, it is unclear to what extent reported associations could be inferred to individuals with prediabetes. Therefore, the purpose of this study was to quantify the relationship between objectively measured physical activity and sedentary time with cardiometabolic health and risk of diabetes.

\subsection{Methods}

A complete description of the methods used in the PREVIEW intervention is provided in chapter 4 .

\subsubsection{Participants and settings}

Participants were recruited between June 2013 and February 2015. Participants were selected through an internet-based pre-screening tool or telephone interview using the Finnish Diabetes Risk Score ${ }^{315}$. A total of 15,611 individuals were contacted for pre-screening. Potential participants were sent a written description of the trial, given verbal information at the study site and signed informed consent prior to a laboratory screening. The laboratory screening was attended by 5,472 participants and included assessment of body mass, stature, resting blood pressure (BP), and a 2-hour OGTT ${ }^{54}$. Glucose concentrations were analysed at each study site (HemoCue ${ }^{\mathrm{TM}}$, Angelholm, Sweden; Reflotron ${ }^{\mathrm{TM}}$, Roche diagnostics, Switzerland; or EML105 Radiometer, Copenhagen) to identify people with pre-existing diabetes. At the end of this process, 2,326 participants met the inclusion criteria. 
Figure 5. 1 Enrolment

\begin{tabular}{|c|c|}
\hline Pre-screened $(\mathrm{n}=15,611)$ & \multirow{2}{*}{$\begin{array}{l}\text { Not eligible }(\mathrm{n}=10,139) \text { Diabetes risk } \\
\text { score }<12 \text { and or did not meet inclusion } \\
\text { criteria }\end{array}$} \\
\hline & \\
\hline $\begin{array}{l}\text { Attended Laboratory Screening } \\
(\mathrm{n}=5,472)\end{array}$ & $\begin{array}{l}\text { Excluded }(\mathrm{n}=3,146) \text { Did not meet } \\
\text { inclusion criteria } 2,890 \text { of these, } 2,837 \text { not } \\
\text { pre-diabetic. }\end{array}$ \\
\hline
\end{tabular}

Eligible for weight loss phase $(n=2,326)$

\subsubsection{Statistical analysis}

Descriptive statistics (mean \pm Standard Deviation; SD) were calculated for continuous variables and frequencies (\%) for categorical variables.

Two-fifths of the participants had a missing value on at least one variable, HOMA-IR was missing in $7.8 \%$ of all cases, while $17 \%$ of values were missing for the accelerometer values (CPM, MVPA, and sedentary time). Multiple imputation with fully conditional specification model (Markov chain Monte Carlo) was used to impute missing values. All variables were included in the imputation and all variables with skewed distribution were $\log _{10}$ or square root transformed prior to imputation ${ }^{316}$. Ten multiple imputed data sets were generated and pooled estimates were reported. Due to their positively skewed distribution, HDL-C, triglycerides hs-CRP, and waist circumference were logarithmically transformed $\left(\log _{10}\right)$ while HOMA-IR was square root transformed.

Separate multiple linear regression models were performed to test the independent association between MVPA, CPM and sedentary time with cardiometabolic health markers (HOMA-IR, fasting insulin, FPG, $2 \mathrm{~h}$ glucose, $\mathrm{HbA}_{1 \mathrm{c}}$, waist circumference, triglycerides, total C, HDL-C and LDL-C) and CRP while adjusting for potential confounders.

Associations have previously been reported between cardio-metabolic risk factors and age, sex, ethnicity, smoking status, sleep, education, household income and body fat ${ }^{317318}$. Therefore, these factors were included in all models 
as potential confounders. Additionally, to account for variation in accelerometer wear time and any variation between intervention sites, each model was also adjusted for wear time and intervention site.

Model 1 was adjusted for age, sex, ethnicity (Caucasian, Asian, Black, Arabic, Hispanic or other), smoking (daily, less than weekly, no smoker), accelerometer wear time, intervention centre, sleep duration, body fat \%, education level (no formal education, primary/junior school, secondary school, secondary vocational education, higher vocational education or university education) and household income (less than $£ 9360, £ 9360$ - $£ 12,479.99, £ 12,480-£ 15,599.99, £ 15,600$ $£ 18,719.99, £ 18,720-£ 22,879.99, £ 22,880-£ 27,559.99, £ 27,560-£ 32,759.99$, $£ 32,760-£ 41,079.99, £ 41,080-£ 53,559.99, £ 53,560$ or more per year). Model 2 was additionally adjusted for sedentary time while MVPA was the main exposure variable or for MVPA when sedentary time was the main exposure variable. Each model was run with and without the independent variable of interest (MVPA, CPM and sedentary time) to isolate the contribution to the explained variance after controlling for confounding variables.

Multicollinearity was controlled for by calculating the Spearman's correlation coefficients between the different accelerometer derived variables (Table 1.1) and by calculating the variance inflation factors. Due to the strong correlation between MVPA and CPM, these two variables were not used in the same model. Sedentary time and CPM were also strongly correlated hence it was not possible to control for sedentary time when CPM was the dependant variable. In contrast, the correlation between sedentary time and MVPA was weak allowing us to control sedentary time when MVPA was the dependent variable. A variance inflation factor $<4$ confirmed that multicollinearity was not a concern ${ }^{319}$

Sex, age $(<45.9,46-54.9,>55$ years $)$, BMI and intervention centre differences in the associations between MVPA, sedentary time, or CPM and each cardiometabolic risk factor were tested for by adding interaction terms to the model. To examine if associations between MVPA, sedentary time or CPM and cardiometabolic risk factor differed by pre-diabetes status (IGT, IFG or both) two way interactions were added to the model. 
Square root and log-transformations were directly compared across cardiometabolic markers, and results of the linear regression analysis are presented as standardised beta coefficients. Data were analysed using the Statistical Package for the Social Sciences version 22.0 (SPSS Inc., Chicago, IL, USA) and alpha was set to $\mathrm{p}<.05$.

\subsubsection{Results}

Table 1. 1 Spearman's correlation matrix of accelerometer variables

\begin{tabular}{|c|c|c|c|c|}
\hline & Sleep & ST & MVPA & CPM \\
\hline Sleep & 1 & $\begin{array}{l}r=-.356 \\
(p<0.005)\end{array}$ & $\begin{array}{l}r=-.096 \\
(p<0.005)\end{array}$ & $\begin{array}{l}r=-.063 \\
(p=0.008)\end{array}$ \\
\hline ST & $\begin{array}{l}r=-.356 \\
(p<0.005)\end{array}$ & 1 & $\begin{array}{l}r=-.286 \\
(p<0.005)\end{array}$ & $\begin{array}{l}r=-.627 \\
(p<0.005)\end{array}$ \\
\hline MVPA & $\begin{array}{l}r=-.096 \\
(p<0.005)\end{array}$ & $\begin{array}{l}r=-.286 \\
(p<0.005)\end{array}$ & 1 & $\begin{array}{l}r=.843 \\
(p<0.005)\end{array}$ \\
\hline CPM & $\begin{array}{l}r=-.063 \\
(p=0.008)\end{array}$ & $\begin{array}{l}r=-.627 \\
(p<0.005)\end{array}$ & $\begin{array}{l}r=.843 \\
(p<0.005)\end{array}$ & 1 \\
\hline
\end{tabular}

Table 1.2 displays the descriptive physical and biochemical characteristics of the 2,326 participants ( $32 \%$ male, age $52.2 \pm 11.5$ years). According to the OGTT laboratory data, $43 \%$ of all included participants had isolated IFG at baseline, $5 \%$ had isolated IGT and 38\% had both IGT and IFG. Waking accelerometer wear time was $928.40 \pm 83.37$ and $933.10 \pm 83.85$ minutes/day for men and women respectively. Mean sedentary time was $617.54 \pm 98.06$ for men and $579.46 \pm 91.76 \mathrm{~min} /$ day for women. Mean MVPA was $31.58 \pm 20.62 \mathrm{~min} /$ day for men and $26.18 \pm 17.03 \mathrm{~min} /$ day for women. Only $50 \%$ of participants met the recommended guidelines of 30 minutes of MVPA per day ${ }^{320}$. Tables $1.3,1.4$ and 1.5 show the standardized regression coefficients of CPM, MVPA, and sedentary time with the cardio-metabolic risk factors. 
Table 1. 2 Metabolic and physical activity characteristics of adults from the PREVIEW study

\begin{tabular}{|c|c|c|}
\hline Characteristics & Female $(n=1570)$ & Male $(n=755)$ \\
\hline Age (years) & $51.6 \pm 11.6$ & $53.5 \pm 11.6$ \\
\hline Height (m) & $1.64 \pm 0.07$ & $1.77 \pm 0.07$ \\
\hline Weight (kg) & $95.88 \pm 20.33$ & $108.87 \pm 20.98$ \\
\hline BMI $\left(\mathrm{kg} \cdot \mathrm{m}^{2}\right)$ & $35.70 \pm 6.76$ & $34.55 \pm 6.01$ \\
\hline Fat $(\%)$ & $46.45 \pm 5.85$ & $36.67 \pm 6.92$ \\
\hline Waist (cm) & $107.7 \pm 14.6$ & $116.8 \pm 14.5$ \\
\hline Systolic BP (mmHg) & $126.98 \pm 5.88$ & $133.10 \pm 14.8$ \\
\hline Diastolic BP (mmHg) & $77.07 \pm 11.15$ & $80.88 \pm 9.96$ \\
\hline Fasting insulin $\left(\mathrm{mU} \cdot \mathrm{l}^{-1}\right)$ & $12.53 \pm 6.54$ & $14.03 \pm 6.68$ \\
\hline Fasting plasma glucose $\left(\mathrm{mmol} \cdot \mathrm{l}^{-1}\right)$ & $6.08 \pm 0.67$ & $6.33 \pm 0.66$ \\
\hline $2 \mathrm{~h}$ plasma glucose $\left(\mathrm{mmol} \cdot \mathrm{l}^{-1}\right)$ & $7.64 \pm 2.21$ & $7.73 \pm 2.24$ \\
\hline $\mathrm{HbA}_{1 \mathrm{c}}\left(\mathrm{mmol} \cdot \mathrm{l}^{-1}\right)$ & $36.61 \pm 4.03$ & $36.73 \pm 4.06$ \\
\hline $\mathrm{HbA}_{1 \mathrm{c}}(\%)$ & $5.50 \pm 0.37$ & $5.51 \pm 0.37$ \\
\hline HOMA-IR & $3.44 \pm 1.90$ & $4.00 \pm 2.01$ \\
\hline Triglycerides $\left(\mathrm{mmol} \cdot \mathrm{l}^{-1}\right)$ & $1.45 \pm 0.77$ & $1.62 \pm 0.82$ \\
\hline Total cholesterol $\left(\mathrm{mmol} \cdot \mathrm{l}^{-1}\right)$ & $5.25 \pm 0.99$ & $5.03 \pm 0.97$ \\
\hline HDL-cholesterol $\left(\mathrm{mmol} \cdot \mathrm{l}^{-1}\right)$ & $1.33 \pm 0.29$ & $1.15 \pm 0.23$ \\
\hline LDL-cholesterol $\left(\mathrm{mmol} \cdot \mathrm{l}^{-1}\right)$ & $3.28 \pm 0.84$ & $3.16 \pm 0.85$ \\
\hline $\mathrm{CRP}(\mathrm{mg} / \mathrm{l})\left(\mathrm{mg} \cdot \mathrm{l}^{-1}\right)$ & $4.81 \pm 4.02$ & $3.46 \pm 3.35$ \\
\hline \multicolumn{3}{|l|}{ Accelerometer variables } \\
\hline Waking wear time (minutes $\cdot d a y^{-1}$ ) & $933.10 \pm 83.85$ & $928.40 \pm 83.37$ \\
\hline Sleep (minutes $\cdot d a y^{-1}$ ) & $474.69 \pm 80.26$ & $471.83 \pm 85.16$ \\
\hline Sedentary $\left(\right.$ minutes $\left.\cdot d a y^{-1}\right)$ & $579.46 \pm 91.76$ & $617.54 \pm 98.06$ \\
\hline Light $\left(\right.$ minutes $\left.\cdot d a y^{-1}\right)$ & $320.84 \pm 82.62$ & $280.12 \pm 78.20$ \\
\hline Moderate (minutes $\cdot$ day $y^{-1}$ ) & $25.30 \pm 6.88$ & $30.33 \pm 20.33$ \\
\hline MVPA $\left(\right.$ minutes $\left.\cdot d a y^{-1}\right)$ & $26.18 \pm 17.03$ & $31.58 \pm 20.62$ \\
\hline CPM $\left(\right.$ counts $^{\prime}$ minute $\left.{ }^{-1}\right)$ & $294.30 \pm 96.77$ & $297.98 \pm 17.28$ \\
\hline \multicolumn{3}{|l|}{ Ethnicity } \\
\hline Caucasian (\%) & 86.02 & 89.9 \\
\hline Asian (\%) & 2.66 & 2.71 \\
\hline Black (\%) & 1.82 & 1.2 \\
\hline Arabic (\%) & 0.2 & 0.3 \\
\hline Hispanic (\%) & 2.33 & 1.38 \\
\hline Other (\%) & 6.61 & 4.25 \\
\hline \multicolumn{3}{|l|}{ Smoking } \\
\hline Yes (Daily) (\%) & 10.72 & 9.57 \\
\hline Sometimes (less than weekly) (\%) & 3.3 & 2.93 \\
\hline No $(\%)$ & 85.98 & 87.48 \\
\hline
\end{tabular}


Table 1.2 Continued

\begin{tabular}{lll}
\hline $\begin{array}{l}\text { Characteristics } \\
\text { Education }\end{array}$ & Female $(n=1570)$ & Male $(n=755)$ \\
No formal education (\%) & 0.5 & -- \\
Primary/junior school (\%) & 2.1 & 2.8 \\
Secondary school (\%) & 15.1 & 14.2 \\
Secondary vocational education (\%) & 17.7 & 19.2 \\
Higher vocational education (\%) & 16.4 & 18.6 \\
University education & 40.5 & 35.6 \\
Other & 7.6 & 9.7 \\
Household income (per year) & & \\
less than $£ 9360,(\%)$ & 8.8 & 5.3 \\
$£ 9360$ - £12,479.99 (\%) & 5.8 & 2.9 \\
$£ 12,480$ - $£ 15,599.99(\%)$ & 5.1 & 2.5 \\
$£ 15,600-£ 18,719.99(\%)$ & 6.5 & 3.5 \\
$£ 18,720$ - $22,879.99(\%)$ & 5.7 & 4.6 \\
$£ 22,880$ - $£ 27,559.99(\%)$ & 8.1 & 5.9 \\
$£ 27,560$ - $£ 32,759.99(\%)$ & 10.6 & 8.1 \\
$£ 32,760-£ 41,079.99(\%)$ & 11.2 & 10.9 \\
$£ 41,080$ - £53,559.99 (\%) & 13.4 & 14.2 \\
$£ 53,560$ or more (\%) & 24.9 & 42 \\
\hline
\end{tabular}

Data are mean \pm SD unless otherwise stated

Table 1.3, shows the standardized regression coefficients of CPM, with the cardio-metabolic risk factors. After adjusting for age, sex, ethnicity, smoking, accelerometer wear time, intervention centre, sleep duration, body fat $\%$, education level and household income, CPM had significant inverse associations with HOMA-IR, waist circumference, fasting insulin, 2-hour glucose, triglycerides and CRP and a positive association with HDL-cholesterol. 
Table 1. 3 Associations between total physical activity and cardio-metabolic risk factors

\begin{tabular}{|c|c|c|c|c|c|}
\hline Characteristics & $B$ & $\begin{array}{l}\text { 95\% Confidence } \\
\text { Interval }\end{array}$ & $p$ & $R^{2}$ & $\begin{array}{c}R^{2} \\
(C P M)\end{array}$ \\
\hline Waist $(\mathrm{cm})$ & -0.179 & $(-0.224,-0.134)$ & & 0.2057 & 0.030 \\
\hline Systolic BP (mmHg) & -0.033 & $(-0.074,0.007)$ & & 0.1927 & 0.001 \\
\hline Diastolic BP (mmHg) & -0.02 & $(-0.057,0.017)$ & & 0.3448 & 0.001 \\
\hline Fasting insulin $\left(\mathrm{mU} \cdot \mathrm{l}^{-1}\right)$ & -0.139 & $(-0.183,-0.096)$ & $* * *$ & 0.1293 & 0.017 \\
\hline Fasting glucose $\left(\mathrm{mmol} \cdot \mathrm{l}^{-1}\right)$ & -0.028 & $(-0.074,0.018)$ & & 0.1647 & 0.001 \\
\hline $2 \mathrm{~h}$ plasma glucose $\left(\mathrm{mmol} \cdot l^{-1}\right)$ & -0.088 & $(-0.131,-0.045)$ & $* * *$ & 0.1139 & 0.007 \\
\hline $\mathrm{HbA}_{1 \mathrm{c}}(\%)$ & -0.049 & $(-0.098,-0.001)$ & & 0.1337 & 0.003 \\
\hline HOMA-IR & -0.151 & $(-0.194,-0.107)$ & *** & 0.1306 & 0.020 \\
\hline Triglycerides $\left(\mathrm{mmol} \cdot \mathrm{l}^{-1}\right)$ & -0.117 & $(-0.162,-0.071)$ & *** & 0.0812 & 0.012 \\
\hline Total cholesterol $\left(\mathrm{mmol} \cdot \mathrm{l}^{-1}\right)$ & -0.007 & $(-0.053,0.038)$ & & 0.095 & 0.00 \\
\hline $\operatorname{HDL}\left(\mathrm{mmol} \cdot \mathrm{l}^{-1}\right)$ & 0.088 & $(0.048,0.129)$ & $* * *$ & 0.1856 & 0.007 \\
\hline $\operatorname{LDL}\left(\mathrm{mmol} \cdot \mathrm{l}^{-1}\right)$ & 0.002 & $-0.045,0.049)$ & & 0.0566 & 0.000 \\
\hline $\mathrm{CRP}\left(\mathrm{mg} \cdot \mathrm{l}^{-1}\right)$ & -0.104 & $(-0.146,-0.062)$ & *** & 0.2657 & 0.010 \\
\hline
\end{tabular}

Adjusted for age, sex, ethnicity, smoking, household income, education level, body fat $\%$, wear time, sleep time and intervention centre. $* * * \mathrm{P}<0.00$. BP, Blood Pressure, $\mathrm{HbA}_{1 \mathrm{c}}$, Glycated haemoglobin A1c, HOMA-IR, Homeostatic Model Assessment Insulin Resistance, HDL, High-Density Lipoprotein, LDL, Low-Density Lipoprotein, CRP, C-reactive protein.

Table 1.4, shows the standardized regression coefficients for MVPA with the cardio-metabolic risk factors. In model 1, adjusted for age, sex, ethnicity, smoking, accelerometer wear time, intervention centre, sleep duration, body fat $\%$, education level and household income, MVPA was significantly and negatively associated with HOMA-IR, waist circumference, fasting insulin, $2 \mathrm{~h}$ glucose, triglycerides and CRP, and positively associated with HDL-cholesterol. After adjusting for sedentary time in model 2, the association with HDLcholesterol was lost. Associations with other biochemical factors were slightly attenuated but all remained significant. 
Table 1. 4 Associations between MVPA (minutes $\cdot d a y^{-1}$ ) and cardio-metabolic risk factors

\begin{tabular}{|c|c|c|c|c|c|}
\hline Characteristics & $B$ & $\begin{array}{c}95 \% \text { Confidence } \\
\text { Interval }\end{array}$ & $p$ & $R^{2}$ & $\begin{array}{c}\boldsymbol{R}^{2} \\
(M V P A)\end{array}$ \\
\hline \multicolumn{6}{|l|}{ Model 1} \\
\hline Waist (cm) & -0.177 & $(-0.122,-0.134)$ & ${ }^{* * * *}$ & 0.2046 & 0.029 \\
\hline Systolic BP (mmHg) & -0.005 & $(-0.047,0.083)$ & & 0.1915 & 0.000 \\
\hline Diastolic BP (mmHg) & -0.007 & $(-0.044,0.031)$ & \multirow[b]{2}{*}{${ }^{* * *}$} & 0.3441 & 0.000 \\
\hline Fasting insulin $\left(\mathrm{mU} \cdot \mathrm{l}^{-1}\right)$ & -0.115 & $(-0.158,-0.072)$ & & 0.1237 & 0.012 \\
\hline Fasting glucose $\left(\mathrm{mmol} \cdot 1^{-1}\right)$ & -0.028 & $(-0.072,0.017)$ & \multirow[b]{2}{*}{ ** } & 0.1645 & 0.000 \\
\hline $2 \mathrm{~h}$ plasma glucose $\left(\mathrm{mmol} \cdot \mathrm{l}^{-1}\right)$ & -0.069 & $(-0.112,-0.025)$ & & 0.1108 & 0.004 \\
\hline $\mathrm{HbA}_{1 \mathrm{c}}(\%)$ & -0.046 & $(-0.096,0.004)$ & \multirow[b]{2}{*}{ *** } & 0.1334 & 0.002 \\
\hline HOMA-IR & -0.122 & $(-0.166,-0.078)$ & & 0.1235 & 0.013 \\
\hline Triglycerides $\left(\mathrm{mmol} \cdot \mathrm{l}^{-1}\right)$ & -0.091 & $(-0.138,-0.044)$ & *** & 0.0762 & 0.007 \\
\hline Total cholesterol $\left(\mathrm{mmol} \cdot \mathrm{l}^{-1}\right)$ & -0.01 & $(-0.056,0.035)$ & \multirow[b]{2}{*}{ * } & 0.0952 & 0.000 \\
\hline $\operatorname{HDL}\left(\mathrm{mmol} \cdot \mathrm{l}^{-1}\right)$ & 0.055 & $(0.009,0.101)$ & & 0.1808 & 0.003 \\
\hline $\operatorname{LDL}\left(\mathrm{mmol} \cdot \cdot^{-1}\right)$ & 0.002 & $(-0.044,0.048)$ & \multirow[b]{2}{*}{ *** } & 0.0567 & 0.000 \\
\hline $\mathrm{CRP}\left(\mathrm{mg} \cdot \mathrm{l}^{-1}\right)$ & -0.086 & $(-0.127,-0.045)$ & & 0.262 & 0.006 \\
\hline \multicolumn{6}{|l|}{ Model 2} \\
\hline Waist (cm) & -0.127 & $(-0.173,-0.081)$ & \multirow[t]{3}{*}{ **** } & 0.2215 & 0.013 \\
\hline Systolic BP (mmHg) & 0.02 & $(-0.026,0.067)$ & & 0.1961 & 0.000 \\
\hline Diastolic BP (mmHg) & 0.011 & $(-0.03,0.053)$ & & 0.3467 & 0.000 \\
\hline \multicolumn{6}{|l|}{ Fasting Insulin $\left(\mathrm{mU} \cdot \mathrm{l}^{-1}\right)$} \\
\hline main effect & -0.078 & $(-0.127,-0.03)$ & ** & 0.1337 & 0.005 \\
\hline 25-45.9 years(reference) & - & - & - & - & \\
\hline $46.0-54.9$ years & 0.025 & $(-0.120,0.170)$ & \multirow[b]{2}{*}{ * } & 0.1313 & - \\
\hline $55.0-71$ years & -0.098 & $(-0.195,-0.001)$ & & 0.1313 & - \\
\hline Fasting glucose $\left(\mathrm{mmol} \cdot 1^{-1}\right)$ & -0.014 & $(-0.063,0.035)$ & \multirow[b]{2}{*}{ * } & 0.1659 & 0.000 \\
\hline $2 \mathrm{~h}$ plasma glucose $\left(\mathrm{mmol} \cdot \cdot^{-1}\right)$ & -0.053 & $(-0.1,-0.006)$ & & 0.1129 & 0.002 \\
\hline $\mathrm{HbA}_{1 \mathrm{c}}(\%)$ & -0.036 & $(-0.095,0.023)$ & \multirow{4}{*}{$\begin{array}{l}* * \\
* *\end{array}$} & 0.1345 & 0.001 \\
\hline HOMA-IR & -0.08 & $(-0.129,-0.031)$ & & 0.1368 & 0.005 \\
\hline Triglycerides $\left(\mathrm{mmol} \cdot \cdot^{-1}\right)$ & -0.067 & $(-0.117,-0.017)$ & & 0.0804 & 0.004 \\
\hline Cholesterol $\left(\mathrm{mmol} \cdot l^{-1}\right)$ & -0.013 & $(-0.064,0.037)$ & & 0.0953 & 0.000 \\
\hline $\operatorname{HDL}\left(\mathrm{mmol} \cdot \mathrm{l}^{-1}\right)$ & 0.028 & $(-0.081,0.383)$ & \multirow[b]{3}{*}{$* *$} & 0.1867 & 0.001 \\
\hline $\operatorname{LDL}\left(\mathrm{mmol}^{-1} \mathrm{l}^{-1}\right)$ & 0 & $(-0.053,-0.052)$ & & 0.0572 & 0.000 \\
\hline $\mathrm{CRP}\left(\mathrm{mmol} \cdot \mathrm{l}^{-1}\right)$ & -0.061 & $(-0.108,-0.015)$ & & 0.2665 & 0.003 \\
\hline
\end{tabular}

Modell adjusted for age, sex, ethnicity, smoking, household income, education level, body fat $\%$, wear time, sleep time, and intervention centre. Model 2 additionally adjusted for sedentary time. ${ }^{*} \mathrm{P}<0.05$; $* * \mathrm{P}<0.01 ; * * * \mathrm{P}<0.00$. BP, Blood Pressure, $\mathrm{HbA}_{1 c}$, Glycated haemoglobin A1c, HOMA-IR, Homeostatic Model Assessment Insulin Resistance, HDL, High-Density Lipoprotein, LDL, Low-Density Lipoprotein, CRP, C-reactive protein.

Table 1.5, shows the standardized regression coefficients for sedentary time with the cardio-metabolic risk factors. In model 1, adjusted for age, sex, ethnicity, smoking, accelerometer wear time, intervention centre, sleep duration, body fat $\%$, education level and household income, sedentary time was positively associated with waist circumference, systolic BP, diastolic BP, mean arterial 
pressure, fasting insulin, $2 \mathrm{~h}$ glucose, HOMA-IR, triglycerides and CRP, and negatively associated HDL. After adjusting for MVPA in model 2, associations with 2-hour plasma glucose were no longer significant. Fasting plasma glucose, total cholesterol and LDL-cholesterol were not associated with any of the exposure variables. Two-way interactions indicate that associations between MVPA and fasting insulin were greater in the older age group (Table 1.3). No significant sex, centre, or BMI interactions were observed between measures of physical activity or sedentary time and any cardio-metabolic risk factors. Two way interactions between MVPA, sedentary time or CPM and pre-diabetes status were not significant indicating that associations did not differ significantly between participants with isolated IFG, IGT or both. 
Table 1. 5 Associations between Sedentary time (minutes $\cdot d a y^{-1}$ ) and cardio-metabolic

\begin{tabular}{|c|c|c|c|c|c|}
\hline Characteristics & $B$ & $\begin{array}{l}\text { 95\% Confidence } \\
\text { Interval }\end{array}$ & $p$ & $R^{2}$ & $\underset{(S E D)}{R_{(2)}^{2}}$ \\
\hline \multicolumn{6}{|l|}{ Model 1} \\
\hline Waist (cm) & 0.215 & $(0.146,0.267)$ & *** & 0.2088 & 0.033 \\
\hline Systolic BP (mmHg) & 0.078 & $(0.026,0.131)$ & \multirow{2}{*}{$\begin{array}{l}* * \\
*\end{array}$} & 0.1957 & 0.004 \\
\hline Diastolic BP (mmHg) & 0.057 & $(0.007,0.106)$ & & 0.3464 & 0.002 \\
\hline Fasting insulin $\left(\mathrm{mU} \cdot \mathrm{l}^{-1}\right)$ & 0.155 & $(0.092,0.219)$ & $* * *$ & 0.1291 & 0.017 \\
\hline Fasting glucose $\left(\mathrm{mmol} \cdot \cdot^{-1}\right)$ & 0.052 & $-0.004,0.108)$ & \multirow[b]{2}{*}{ * } & 0.1656 & 0.000 \\
\hline $2 \mathrm{~h}$ plasma glucose $\left(\mathrm{mmol} \cdot \mathrm{l}^{-1}\right)$ & 0.072 & $(0.015,0.129)$ & & 0.1106 & 0.004 \\
\hline $\mathrm{HbA}_{1 \mathrm{c}}(\%)$ & 0.047 & $(-0.022,0.117)$ & \multirow[b]{2}{*}{$* * *$} & 0.1332 & 0.002 \\
\hline HOMA-IR & 0.175 & $(0.114,0.236)$ & & 0.1316 & 0.021 \\
\hline Triglycerides $\left(\mathrm{mmol} \cdot \cdot^{-1}\right)$ & 0.106 & $(0.052,0.16)$ & $* * *$ & 0.0766 & 0.008 \\
\hline Total cholesterol $\left(\mathrm{mmol} \cdot \mathrm{l}^{-1}\right)$ & -0.006 & $(-0.062,0.051)$ & \multirow[b]{2}{*}{ *** } & 0.0953 & 0.000 \\
\hline $\operatorname{HDL}\left(\mathrm{mmol} \cdot \mathrm{l}^{-1}\right)$ & -0.103 & $(-0.165,-0.042$ & & 0.1859 & 0.007 \\
\hline $\operatorname{LDL}\left(\mathrm{mmol} \cdot \cdot^{-1}\right)$ & -0.007 & $(-0.062,0.048)$ & \multirow[b]{2}{*}{ ** } & 0.0568 & 0.000 \\
\hline $\begin{array}{l}\text { CRP }\left(\mathrm{mg} \cdot 1^{-1}\right) \\
\text { Model } 2\end{array}$ & 0.106 & $(0.039,0.172)$ & & 0.2632 & 0.008 \\
\hline Waist (cm) & 0.165 & $(0.109,0.221)$ & \multirow{4}{*}{$\begin{array}{l}* * * \\
* * \\
* \\
* \\
* *\end{array}$} & 0.2215 & 0.013 \\
\hline Systolic BP (mmHg) & 0.086 & $(0.028,0.143)$ & & 0.1961 & 0.000 \\
\hline Diastolic BP (mmHg) & 0.061 & $(0.006,0.116)$ & & 0.3467 & 0.000 \\
\hline Fasting insulin $\left(\mathrm{mU} \cdot \mathrm{l}^{-1}\right)$ & 0.126 & $(0.055,0.198)$ & & 0.1337 & 0.005 \\
\hline Fasting glucose $\left(\mathrm{mmol} \cdot \cdot^{-1}\right)$ & 0.047 & $(-0.014,0.108)$ & \multirow{5}{*}{$\begin{array}{l}* * * \\
* *\end{array}$} & 0.1659 & 0.000 \\
\hline $2 \mathrm{~h}$ plasma glucose $\left(\mathrm{mmol} \cdot{ }^{-1}\right)$ & 0.053 & $(-0.009,0.114)$ & & 0.1129 & 0.002 \\
\hline $\mathrm{HbA}_{1 \mathrm{c}}(\%)$ & 0.034 & $(-0.045,0.113)$ & & 0.1345 & 0.002 \\
\hline HOMA-IR & 0.145 & $(0.077,0.213)$ & & 0.1368 & 0.005 \\
\hline Triglycerides $\left(\mathrm{mmol} \cdot \cdot^{-1}\right)$ & 0.08 & $(0.023,0.138)$ & & 0.0804 & 0.004 \\
\hline Total cholesterol $\left(\mathrm{mmol} \cdot 1^{-1}\right)$ & -0.011 & $(-0.074,0.052)$ & & 0.0953 & 0.000 \\
\hline $\mathrm{HDL}\left(\mathrm{mmol} \cdot \cdot^{-1}\right)$ & -0.093 & $(-0.163,-0.022)$ & & 0.1867 & 0.001 \\
\hline $\operatorname{LDL}\left(\mathrm{mmol} \cdot \cdot^{-1}\right)$ & -0.007 & $(-0.069,0.055)$ & \multirow[b]{2}{*}{ * } & 0.0572 & 0.000 \\
\hline $\operatorname{CRP}\left(\mathrm{mg} \cdot \mathrm{l}^{-1}\right)$ & 0.083 & $(0.01,0.156)$ & & 0.2665 & 0.003 \\
\hline
\end{tabular}

Modell adjusted for age, sex, ethnicity, smoking, household income, education level, body fat $\%$, wear time, sleep time, and intervention centre. Model 2 additionally adjusted for MVPA. $* \mathrm{P}<0.05 ; * * \mathrm{P}<0.01$; $* * * \mathrm{P}<0.001 \mathrm{BP}$, Blood Pressure, $\mathrm{HbA}_{1 \mathrm{c}}$, Glycated haemoglobin A1c, HOMA-IR, Homeostatic Model Assessment Insulin Resistance, HDL, High-Density Lipoprotein, LDL, Low-Density Lipoprotein, CRP, C-reactive protein.

\subsection{Discussion}

This study investigated the associations between objectively measured physical activity and sedentary time and metabolic variables in a worldwide sample of overweight and obese adults $\left(\mathrm{BMI}>25 \mathrm{~kg} / \mathrm{m}^{2}\right)$ with pre-diabetes confirmed through an OGTT ${ }^{52}$. To our knowledge this is the first international investigation of associations between objectively measured physical activity and sedentary time with cardio-metabolic risk factors in a population that exclusively meet the criteria for pre-diabetes (IFG 5.6-6.9 or IGT $7.8-11\left(\mathrm{mmol} \cdot \mathrm{l}^{-1}\right)$. 
Previous studies conducted in high risk populations have included participants based on a risk score questionnaire ${ }^{148}$, family history of type 2 diabetes ${ }^{177,191}$ or participants with diabetes and pre-diabetes have been combined ${ }^{192}$. Consequently, physical characteristics and metabolic parameters reported in these studies varied substantially from the present study. In our population of participants with pre-diabetes, MVPA was negatively associated with HOMAIR, waist circumference, fasting insulin, $2 \mathrm{~h}$ glucose, triglycerides, and CRP after accounting for potential confounders, sleep duration and sedentary time. This study also demonstrated that total physical activity volume (counts $\cdot$ minute $^{-1}$ ) at least as strongly associated with the aforementioned risk factors as MVPA.

Before controlling for sedentary time in model 2, total physical activity volume accounted for greater variance in many cardio-metabolic risk factors than MVPA. While many of these differences may be equivocal, CPM accounted for $35 \%$ more variance in HOMA-IR than MVPA. Hence, it appears that in this population although both are significant, the accumulation of total physical activity over a day is at least as strong an indicator of insulin resistance and some related cardio-metabolic risk factors as MVPA.

Previous studies in populations with a family history of diabetes and newly diagnosed diabetes, have shown that total energy expenditure spent on physical activity ${ }^{192}$, counts $\cdot$ minute $^{-1} 191$ and MVPA ${ }^{177}$ were negatively associated with waist circumference, fasting serum triglycerides, systolic BP, fasting plasma glucose, fasting plasma insulin, HOMA-IR and a clustered metabolic risk score. In agreement with our findings, Ekelund et al. ${ }^{191}$, reported that total counts ·day 1 was more strongly associated with clustered risk and individual cardiometabolic risk factors than MVPA. In the general population, Balkau and colleagues ${ }^{321}$ reported associations between MVPA and total physical activity with insulin resistance using the gold standard clamp technique for determining insulin sensitivity. However, after adjusting for total physical activity, associations with MVPA were lost ${ }^{321}$. These findings, in keeping with the present study would support the hypothesis that the accumulation of total physical activity volume accounts for greater variance in insulin resistance and some related cardio-metabolic risk factors than MVPA. The present study also demonstrated that after controlling for confounders, sedentary time is positively 
associated with waist circumference, systolic BP, diastolic BP, fasting insulin, HOMA-IR, triglycerides, CRP, and HDL-cholesterol independent of time spent in MVPA.

In agreement with our findings, Henson et al ${ }^{148}$ reported positive associations between sedentary time, 2 hour plasma glucose, triglycerides and HDLcholesterol, and independent total physical activity in a population at risk of type 2 diabetes ${ }^{148}$. Similarly, positive associations between sedentary time, waist circumference, insulin, HOMA-IR, and HDL-cholesterol were reported in an older sample with newly diagnosed type 2 diabetes ${ }^{165}$. There is a lack of consensus on the role of sedentary time in pathology of cardio-metabolic risk factors. Some authors have reported that once total energy expenditure is taken into account the association between sedentary time and cardio-metabolic health is lost. These findings suggest that sedentary time reduces total energy expenditure by displacing other activities that are more energy costly ${ }^{321,322}$. Other investigators have found that the mechanism linking sedentary time to glucose metabolism and metabolic health differ from those of physical activity and may be related to static posture and unloading of large skeletal muscle groups when seated 167,323 . Sedentary time is thought to affect glucose homeostasis and lipid metabolism by reducing muscle GLUT-4 content and insulin-stimulated glucose uptake ${ }^{166}$ while also reducing LPL activity leading to impaired triglyceride and HDL cholesterol metabolism ${ }^{167}$.

Further, light activity is associated with marked improvements in cardiometabolic health ${ }^{188}$ while interventions have shown that replacing sedentary time with postural changes such as standing or light ambulatory activity can improve glycaemic control to a greater extent than structured exercise of the same energy cost ${ }^{324}$. In the present analysis, sedentary time was associated with HDL cholesterol, however, associations between MVPA and HDL cholesterol were lost after controlling for sedentary time. Similarly, sedentary time was positively associated with systolic and diastolic blood pressure independent of MVPA while no association was found between CPM or MVPA with either blood pressure variable.

Associations between physical activity, sedentary time and blood pressure variables reported by observation studies have been inconsistent 188,190,192. However, experimental studies in overweight/obese populations have suggested 
that the reduction of sedentary time and interrupting prolonged sedentary bouts with light or moderate activity is associated with improved systolic and diastolic blood pressure ${ }^{325}$.

While physical activity guidelines continue to focus on participation in MVPA 320 , our data demonstrated that in a population with pre-diabetes, total volume of physical activity was as strongly associated with cardio-metabolic health as MVPA. The implications of this finding may be important considering the levels of physical activity in this population. Light activity may be more readily adopted by individuals with pre-diabetes particularly if they are physically inactive, overweight/obese, or reluctant to engage in structured exercise ${ }^{324}$.

The lack of associations observed for all exposure variables with fasting glucose is consistent with previous research ${ }^{148,188}$. This finding reflects the fact that the physical activity predominantly affects peripheral insulin sensitivity, which is responsible for lowering blood glucose levels after an OGTT when most of the glucose is taken up by skeletal muscle ${ }^{48}$. Hence, it may also be expected that associations between insulin resistance and physical activity are stronger in participants with isolated IGT than those with IFG. This was not the case in the present study, possibly owing to the small number of participants $(5 \%)$ with isolated IGT.

The strengths of this study are the implementation of a $24 \mathrm{~h}$ accelerometer wear time protocol that resulted in a greater mean waking wear time (15.5 hours) than many studies of a similar nature ${ }^{148,165,280}$. Longer monitoring duration provides greater reliability of average activity estimates. This approach also allowed an objective assessment of sleep time using an algorithm to detect sleep onset and wake time from a $24 \mathrm{~h}$ waist worn accelerometer trace ${ }^{282}$. This allowed the study team to control for the confounding effects of sleep on cardio-metabolic risk factors ${ }^{318}$.

Type 2 diabetes is high on public health agendas with attention on the prevention or delay of diabetes onset and the management of cardio-metabolic risk factors 326,327. National and international guidelines focus on first identifying high-risk individuals and second controlling modifiable risk factors such as body weight, diet, sedentary time and physical activity, through targeted interventions ${ }^{328,329}$. This study provides new evidence of associations between physical activity and 
sedentary time with cardio-metabolic markers in a population to which the results are most applicable.

Whilst this study has numerous strengths it is also important to acknowledge its limitations. First, the cross-sectional design does not allow insight into the direction of causality between each exposure variable and markers of cardiometabolic health and although we controlled for many potential confounding factors, we did not account for dietary intake or alcohol consumption which may have influenced our results. It is important to note that MVPA, CPM and sedentary time only accounted for a small proportion of the total variance in cardio-metabolic risk factors (0-3\%) indicating that other factors play an important role in determining cardio-metabolic health. Secondly, although accelerometers offer more robust assessments of physical activity than selfreport ${ }^{179}$, they are not without limitation. Hip worn accelerometers capture most movement during locomotion, but cannot account for upper body movement or movement that occurs during activities such as cycling or weight lifting ${ }^{330}$. Furthermore, the accelerometer is removed during water based activities and contact sports. Therefore, physical activity may be underestimated. The use of accelerometer trace to distinguish sedentary time specifies time spent $<100$ CPM but does not capture postural changes. Consequently, low level activities such as sitting and standing will be combined. Given the fixed nature of accelerometerderived variables (sleep, light activity sedentary time and MVPA) as proportions of wake time, time spent in behaviours within the day are inherently collinear; every increase in time spent in one behaviour unavoidably causes a decrease in the time spent in one or a combination of the other behaviours. Thus, it is not possible to include all subcomponents of the day (sleep, sedentary, light activity, MVPA) in a regression model without violating collinearity assumptions. Consequently, in the present study it is not possible to say with certainty that the positive associations observed between cardio-metabolic risk factors and sedentary time are truly independent and not in fact negative associations with light activity. Finally, participants in this study were voluntary recruits to a lifestyle intervention from the 8 study sites. Approximately $50 \%$ of the study sample were between the age of 55-70 years. A greater proportion of older participants was expected as type 2 diabetes risk increases with age and more of 
this age group are retired and have time to participate in a demanding intervention. Therefore, these results may be confined to overweight, obese adults with adults with pre-diabetes.

\subsection{Conclusion}

In conclusion, this study provides new evidence that in a large population of adults with pre-diabetes, objective measures of physical activity and sedentary time are associated with markers of cardio-metabolic health. Furthermore, associations with total physical activity volume are at least as strong as MVPA. Taken together, these findings suggest that replacing sedentary time with light activity may provide a practical approach to improve cardio-metabolic health in a population with low engagement in MVPA. 


\subsection{Thesis Map}

\begin{tabular}{|c|c|c|}
\hline Study & Aims & Findings \\
\hline $\begin{array}{l}\text { 1. } \\
\text { Objectively measured physical } \\
\text { activity and sedentary time are } \\
\text { associated with cardio-metabolic } \\
\text { risk factors in adults with pre- } \\
\text { diabetes: The PREVIEW study. }\end{array}$ & $\begin{array}{l}\text { To measure the relationship } \\
\text { between cardio-metabolic risk } \\
\text { factors and objectively measured } \\
\text { physical activity and sedentary } \\
\text { time. } \\
\mathrm{H}_{0} \text { : The magnitude of the } \\
\text { association between physical } \\
\text { activity and cardio metabolic risk } \\
\text { factors is equal to zero. }\end{array}$ & $\begin{array}{l}\text { In adults with pre-diabetes, } \\
\text { objectively measured physical } \\
\text { activity and sedentary time } \\
\text { were associated with cardio- } \\
\text { metabolic risk markers. Total } \\
\text { physical activity was at least as } \\
\text { strongly associated with } \\
\text { cardio-metabolic risk markers } \\
\text { as MVPA. }\end{array}$ \\
\hline $\begin{array}{l}2 . \\
\text { Correlates of physical activity in } \\
\text { adults with pre-diabetes from } 8 \\
\text { countries: the PREVIEW study. }\end{array}$ & $\begin{array}{l}\text { To assess the correlates of } \\
\text { objectively measured physical } \\
\text { activity in adults with pre-diabetes } \\
\text { in eight countries and to examine } \\
\text { inter-country differences with site- } \\
\text { specific interactions. } \\
\mathrm{H}_{0} \text { : The magnitude of association } \\
\text { between psychosocial variables } \\
\text { and physical activity is equal to } \\
\text { zero. } \\
\mathrm{H}_{0} \text { : There is no difference between } \\
\text { country in the magnitude of } \\
\text { association between psychosocial } \\
\text { variables and physical activity. }\end{array}$ & \\
\hline
\end{tabular}

3.

Lifestyle behaviours associated

with body fat percent in 9-11-year-

old children, a population-based,

cross-sectional study: The

SportsLinx project.

4.

The effectiveness of a child weight management intervention; a mixed methods investigation of child and parent's perceptions of the behaviour change process. 


\section{Study 2: Personal and psychosocial correlates of physical activity in adults with pre-diabetes from 8 countries: the PREVIEW study}

\subsection{Introduction}

Physical activity is a key component in the prevention of type 2 diabetes through its role in weight loss and maintenance and the improvement of insulin sensitivity ${ }^{255}$. Clinical trials have demonstrated that diet and physical activity induced weight loss can prevent, or at least delay, the onset of type 2 diabetes in high-risk individuals ${ }^{21,245}$. Despite the potential benefits of physical activity, most patients with type 2 diabetes, or at the highest risk of developing type 2 diabetes, do not engage in sufficient physical activity to elicit these associated health benefits ${ }^{193}$. The promotion of physical activity in adults with pre-diabetes depends on understanding the underlying influential factors, and consequently theory, which improve the likelihood of achieving the desired behavioural change. Previous literature on the correlates of physical activity in a variety of populations has highlighted three levels of influence: environmental, social and individual. Environmental factors include the availability and accessibility of physical activity opportunities, neighbourhood design and the transport environment. Social factors include familial and peer social support, whereas individual level factors include demographic, biological and psychosocial factors, such as age, sex, ethnicity, education, exercise intention and selfefficacy ${ }^{197}$.

To date, the majority of the research on correlates of physical activity has focussed on the general population, with the interpretation of the limited research conducted in adults at risk of type 2 diabetes ${ }^{210,211}$ severely limited by the reliance on self-reported physical activity, which is inherently susceptible to subjective interpretation and recall bias ${ }^{179}$. Given that BMI, a risk factor for type 2 diabetes, has been shown to moderate correlates of physical activity ${ }^{201}$, it is postulated that individuals at high-risk of type 2 diabetes may have different underlying facilitators and barriers to physical activity. Indeed, psychosocial mechanisms are central to the behaviour change framework underpinning the PREVIEW study, which aims to elicit sustained improvements in diet and physical activity through the manipulation of the psychosocial factors associated 
with diet and physical activity ${ }^{296}$. Furthermore, given that previous research has shown that correlates of physical activity may differ between countries, the interpretation of previous studies is further limited ${ }^{331}$. Indeed, cultural background often drives individual behaviour ${ }^{205}$, however, due to inter-study differences in methodological approaches, questionnaires and tools used to assess the psychosocial attributes and environmental correlates of physical activity, firm conclusions between different countries cannot be made.

Therefore, the aim of this study was to assess the correlates of objectivelymeasured physical activity in adults with pre-diabetes in multiple countries and to examine inter-country differences.

\subsection{Methods}

A complete description of the methods used in the PREVIEW project are provided in chapter 4

\subsubsection{Design and analysis}

In total, $11 \%$ of values were missing; to reduce potential bias, multiple imputation strategies were applied ${ }^{332}$ and an intention to treat (ITT) analyses conducted. A fully conditional specification model (Markov chain Monte Carlo) was used to impute missing values for all variables. Variables with a skewed

distribution were $\log _{10}$ or square-root transformed prior to imputation ${ }^{316}$. Ten multiple imputed datasets were generated and pooled-estimates reported.

Descriptive statistics (mean \pm SD for continuous variables; percentages for categorical variables) were calculated to describe participant characteristics and physical activity levels by country. MVPA was negatively skewed and was square-root transformed for all analyses. Exploratory one-way analysis of variance (ANOVA) and Tukey's post-hoc analyses were conducted to examine differences according to country, age and sex.

As the data were collected in a clustered design from intervention centres in eight countries, it seems likely that participants within a country would be more similar to each other than to participants from other countries due to unmeasured country-level characteristics. To interrogate this possibility, a linear mixedmodel regression was used with country as a random effect. The covariance parameter for the random effect demonstrated a significance value $>0.05$ and the 
$-2 \log$ likelihood of models with and without country as a random effect did not differ significantly $\left(\chi^{2}>0.5\right)$. Therefore, as accounting for the variability between intercepts for each country did not significantly improve the model, country was subsequently treated as a fixed effect.

Multiple linear regression was used to examine the associations between psychosocial variables while controlling socio-demographic covariates and possible confounders. Demographic variables included: age, sex, ethnicity (Caucasian, Asian, Black, Arabic, Hispanic or other), education level (no formal education, primary/junior school, secondary school, secondary vocational education, higher vocational education or university education) and household income (less than $€ 13,200, € 13,200-€ 17,000$, €17,001-€20,500, €20,501$€ 24,200, € 24,201-€ 28,600, € 28,601-€ 33,500, € 33,501-€ 39,100, € 39,101-$ $€ 46,400, € 46,401-€ 52,800, € 52,801$ or more per year), Other potential confounders were smoking (daily, less than weekly, non-smoker), study site, and accelerometer wear-time $\left(\mathrm{min} \cdot \mathrm{day}^{-1}\right)$. Given the diversity of the geographical location of the study sites and seasonal change, the model was additionally adjusted for daylight hours. Demographic variables and all potential confounds were entered into the model in the first block. Psychosocial variables were entered into the model in the second block. In the third block, two-way site by psychosocial variable interaction terms were added to examine whether the independent associations of psychosocial variables with MVPA varied by study site. Non-significant interactions $(p>0.05)$ were removed from the model before adding sex by explanatory variable interactions to examine whether the independent associations of psychosocial variables with MVPA varied by sex or age. Finally, to examine the moderating effect of psychosocial variables on each another, two way interactions were entered between each variable.

Any significant moderators were entered into the model as three-way interactions with site to investigate whether the moderating effects of specific psychosocial variables differed by study site. All significant interaction terms ( $p$ $>0.05)$ were probed by estimating associations at 1 standard deviation below and above the mean value of the moderating variable ${ }^{333}$. For the visual interpretation of interaction effects, predicted values of MVPA were plotted at high and low values of the independent and moderator variables showing the 
predicted relationship between MVPA and the independent variable at different levels of the moderator.

There was independence of residuals, as assessed by a Durbin-Watson statistic of 1.956 and homoscedasticity, as assessed by visual inspection of a plot of standardized residuals versus unstandardized predicted values. A variance inflation factor less than 4 suggested that multi-collinearity did not need to be accounted for in the analysis ${ }^{319}$.

Data were analysed using the Statistical Package for the Social Sciences version 22 (SPSS Inc., Chicago, IL, USA).

\subsection{Results}

Descriptive statistics at baseline for socio-demographic variables, psychosocial variables and daily MVPA for each study site are presented in Table 2.1. Mean age varied significantly across study sites $(F(7,2318)=47.2 ; \mathrm{p}<0.001)$. There were a greater number of female participants at all sites, with the UK $(29 \%$ male) and New Zealand (29\% male) showing the largest discrepancy between sexes. Despite the variation in seasons across countries, daylight hours did not differ significantly during data collection $(F(7,2234)=1.35 ; \mathrm{p}=0.224)$.

Mean BMI varied across study sites $(F(7,2316)=37.7 ; \mathrm{p}<0.001)$. Specifically, the highest and lowest BMI was reported New Zealand $\left(38.4 \pm 6.7 \mathrm{~kg} \cdot \mathrm{m}^{-2}\right)$ and the Netherlands $\left(32.0 \pm 4.1 \mathrm{~kg} \cdot \mathrm{m}^{-2}\right)$ respectively. Tukey's post-hoc analysis revealed that the BMI in New Zealand and Bulgaria was significantly higher than in all other centres $(p<0.01)$, while BMI in the Netherlands was significantly lower than all other centres, except for Finland. 
Table 2. 1 Site-Specific Descriptive Statistics for all Explanatory Variables

\begin{tabular}{|c|c|c|c|c|c|c|}
\hline & $\begin{array}{c}\text { Denmark } \\
n=379\end{array}$ & $\begin{array}{c}\text { Findland } \\
n=289\end{array}$ & $\begin{array}{c}\text { Netherlands } \\
n=203\end{array}$ & $\begin{array}{c}\mathbf{U K} \\
n=264\end{array}$ & $\begin{array}{c}\text { Spain } \\
n=307\end{array}$ & $\begin{array}{c}\text { Bulgar } \\
n=36\end{array}$ \\
\hline Age (mean \pm SD) & $54.2 \pm 10.9$ & $58.2 \pm 8.9$ & $55.6 \pm 10.0$ & $51.6 \pm 12.0$ & $47.5 \pm 10.6$ & $47.8 \pm 12$. \\
\hline Sex $\%$ male & 41 & 47 & 33 & 29 & 37 & 46 \\
\hline $\mathrm{BMI} \mathrm{kg} \cdot \mathrm{m}^{2}($ mean $\pm \mathrm{SD})$ & $34.7 \pm 5.2$ & $32.7 \pm 4.6$ & $32.0 \pm 4.1$ & $35.4 \pm 5.7$ & $34.5 \pm 6.0$ & $37.3 \pm 7.2$ \\
\hline Body fat $\%$ & $45.0 \pm 6.9$ & $39.1 \pm 7.7$ & $41.5 \pm 7.5$ & $45.3 \pm 7.4$ & $41.4 \pm 7.4$ & $42.4 \pm 7.4$ \\
\hline Caucasian \% & 97.9 & 100 & 98.5 & 81.1 & 84.9 & 100 \\
\hline Asian $\%$ & 1.9 & - & 1 & 7.6 & - & - \\
\hline Black \% & - & - & 0.5 & 11 & - & - \\
\hline Arabic \% & - & - & - & 0.4 & - & - \\
\hline Hispanic \% & - & - & - & - & 15.1 & - \\
\hline Ethnicity other \% & 0.3 & - & - & - & & - \\
\hline Smoking \% no & 88.7 & 88.6 & 91.1 & 93.9 & 80.8 & 65.6 \\
\hline Smoking \% sometimes & 4.5 & 4.8 & 4.4 & 1.1 & 5.2 & 2.6 \\
\hline Smoking \% daily & 6.8 & 6.6 & 4.4 & 5 & 13.9 & 31.8 \\
\hline Day light $\left(\min \cdot\right.$ day $\left.^{-1}\right)$ & $763 \pm 138$ & $760 . \pm 142$ & $765 \pm 130$ & $763 \pm 140$ & $763 \pm 129$ & $762 \pm 147$ \\
\hline MVPA (min $\cdot$ day $\left.^{-1}\right)$ & $27 \pm 17$ & $29 \pm 18$ & $30 \pm 20$ & $22 \pm 16$ & $35 \pm 20$ & $31 \pm 17$ \\
\hline Time for PA & $3.1 \pm 0.7$ & $3.3 \pm 0.7$ & $3.1 \pm 0.8$ & $2.9 \pm 0.6$ & $2.9 \pm 0.7$ & $2.9 \pm 0.6$ \\
\hline $\begin{array}{l}\text { Place for PA } \\
\text { Social support }\end{array}$ & $3.5 \pm 0.6$ & $3.6 \pm 0.6$ & $3.4 \pm 0.8$ & $3.2 \pm 0.6$ & $3.3 \pm 0.7$ & $3.1 \pm 0.7$ \\
\hline Friends & $2.0 \pm 0.7$ & $2.2 \pm 0.8$ & $2.1 \pm 0.8$ & $1.9 \pm 0.8$ & $2.4 \pm 0.9$ & $1.9 \pm 0.8$ \\
\hline Family & $2.2 \pm 0.8$ & $2.5 \pm 0.8$ & $2.4 \pm 0.8$ & $2.1 \pm 0.9$ & $2.6 \pm 0.9$ & $2.1 \pm 1.0$ \\
\hline
\end{tabular}


Table 2.1 Continued

\begin{tabular}{|c|c|c|c|c|c|c|}
\hline & $\begin{array}{c}\text { Denmark } \\
n=379\end{array}$ & $\begin{array}{l}\text { Finland } \\
n=289\end{array}$ & $\begin{array}{c}\text { Netherlands } \\
n=203\end{array}$ & $\begin{array}{c}\mathbf{U K} \\
n=264\end{array}$ & $\begin{array}{c}\text { Spain } \\
n=307\end{array}$ & $\begin{array}{c}\mathbf{B u} \\
n\end{array}$ \\
\hline \multicolumn{7}{|l|}{ Discouragement } \\
\hline Friends & $1.2 \pm 0.3$ & $1.1 \pm 0.3$ & $1.2 \pm 0.4$ & $1.2 \pm 0.4$ & $1.2 \pm 0.4$ & $1.3 \pm$ \\
\hline Family & $1.2 \pm 0.4$ & $1.2 \pm 0.4$ & $1.3 \pm 0.4$ & $1.4 \pm 0.5$ & $1.3 \pm 0.5$ & $1.4 \pm$ \\
\hline Inactivity temptations & $3.2 \pm 0.9$ & $3.1 \pm 0.8$ & $3.0 \pm 0.9$ & $3.2 \pm 0.9$ & $2.9 \pm 1.0$ & $3.0 \pm$ \\
\hline Goal disengagement & $3.1 \pm 0.8$ & $2.8 \pm 0.8$ & $2.8 \pm 0.7$ & $2.9 \pm 0.8$ & $2.8 \pm 0.7$ & $2.5 \pm$ \\
\hline Goal reengagement & $3.6 \pm 0.7$ & $3.5 \pm 0.7$ & $3.6 \pm 0.6$ & $3.3 \pm 0.7$ & $3.6 \pm 0.8$ & $3.4 \pm$ \\
\hline Self-efficacy & $2.8 \pm 0.6$ & $3.0 \pm 0.6$ & $2.8 \pm 0.6$ & $2.7 \pm 0.7$ & $2.8 \pm 0.7$ & $2.9 \pm$ \\
\hline Habit strength sitting & $125 \pm 94$ & $137 \pm 93$ & $91 \pm 71$ & $142 \pm 99$ & $103 \pm 91$ & $116 \pm$ \\
\hline Habit strength transport & $145 \pm 128$ & $150 \pm 113$ & $130 \pm 127$ & $200 \pm 123$ & $176 \pm 128$ & $175 \pm$ \\
\hline Intentions to exercise & $5.4 \pm 1.7$ & $5.7 \pm 1.4$ & $6.1 \pm 1.2$ & $5.4 \pm 1.7$ & $5.9 \pm 1.6$ & $5.8 \pm$ \\
\hline $\begin{array}{l}\text { Attribution } \\
\text { Internal/External) }\end{array}$ & $1.9 \pm 1.2$ & $2.0 \pm 1.3$ & $2.53 \pm 1.5$ & $1.9 \pm 1.2$ & $2.0 \pm 1.4$ & $2.4 \pm$ \\
\hline Stable/unstable stable & $4.4 \pm 1.4$ & $3.3 \pm 1.5$ & $3.7 \pm 1.4$ & $3.4 \pm 1.6$ & $3.4 \pm 1.8$ & $4.9 \pm$ \\
\hline Control Others & $4.9 \pm 1.8$ & $3.6 \pm 2$ & $4.0 \pm 1.9$ & $5.2 \pm 1.8$ & $3.0 \pm 1.9$ & $3.8 \pm$ \\
\hline Control Self & $4.9 \pm 1.3$ & $6.4 \pm 1$ & $4.0 \pm 1.6$ & $5.2 \pm 1.8$ & $3.8 \pm 2.1$ & $5.6 \pm$ \\
\hline
\end{tabular}

Mean and standard deviations are presented unless otherwise stated 
The ethnicity of participants was predominantly Caucasian, except for New Zealand, which had a large number of Maori and Pacific participants (44.9\%). In the UK, $11.0 \%$ of the cohort were black; $7.6 \%, 7.2 \%$ and $2.5 \%$ were Asian for the UK, Australia and New Zealand respectively.

Moderate-to-vigorous physical activity was significantly lower in the UK and New Zealand than all other centres $(p<0.01)$, while the highest mean MVPA was in Spain, significantly higher than all other centres $(\mathrm{p}<0.01)$, except for Bulgaria $(\mathrm{p}>0.05)$. No statistically significant difference in physical activity was present between the Netherlands, Finland, Australia and Denmark.

Table 2.2 presents the main effects of the associations between psychosocial variables and MVPA after adjusting for age, sex, ethnicity, smoking, accelerometer wear time, daylight hours, intervention centre, education level and household income. Two-way interactions are presented in Table 2.3. After accounting for socio-demographic and psychosocial variables, discouragement from family, place for physical activity, time for physical activity, goal reengagement, daylight hours and attribution of control by others were not significantly associated with MVPA as a main effect or interaction term and were therefore not included in the final model. The final model significantly predicted the dependent variable $\left(\mathrm{MVPA} ; F(58,2259)=9.754 ; \mathrm{p}=0.0005 ; \mathrm{R}^{2}=\right.$ 0.196). Main effects for self-efficacy and goal disassociation were not significant, however, two-way interactions revealed that self-efficacy was positively associated with MVPA in the UK and Australia, while goal dissociation was positively associated with MVPA in the UK and Finland. 
Table 2. 2 Associations between psychosocial attributes and MVPA

\begin{tabular}{lllll}
\hline & \multicolumn{4}{c}{$(58,2259)=9.754 ; \mathrm{p}=0.0005 ; \mathrm{R}^{2}=0.196$} \\
& & B Coefficients & \multicolumn{2}{c}{$95 \%$ Confidence Interval } \\
& & \multicolumn{4}{c}{$\begin{array}{c}\text { Lower bound } \\
\text { bound }\end{array}$} & $\mathrm{p}$ \\
\hline Constant & 5.119 & 4.863 & 5.375 & $<0.0005$ \\
Intention to Exercise & 0.094 & 0.051 & 0.137 & $<0.0005$ \\
Self-Efficacy & -0.039 & -0.316 & 0.237 & 0.781 \\
Habit strength & -0.001 & -0.002 & -0.001 & $<0.0005$ \\
transport & & & & \\
Goal disengagement & -0.147 & -0.387 & 0.093 & 0.23 \\
Goal reengagement & 0.062 & -0.032 & 0.157 & 0.194 \\
Inactivity temptations & -0.105 & -0.181 & -0.029 & 0.007 \\
Social support family & 0.038 & -0.055 & 0.13 & 0.423 \\
Social support friends & 0.044 & -0.057 & 0.145 & 0.391 \\
\hline
\end{tabular}

This table presents the association between psycho-social variables and MVPA controlling for age, sex, ethnicity, smoking, accelerometer wear time, daylight hours, intervention centre, education level and household income

Main effects for the three dimensions of causal attribution were not associated with MVPA and two-way interactions with centre did not produce significant effects, suggesting that the lack of association was consistent across centres. The main effect for age was negatively associated with MVPA $(p<0.05)$. Age also moderated the relationship between familial social support and MVPA ( $p=$ 0.009). The slope of the associations between familial social support and MVPA (Figure 2.1), was not significant for younger participants $(\beta=-0.034[0.07$, $0.14], \mathrm{p}<0.522)$ but a significant positive association was found for older participants $(\beta=0.15[0.26,0.04], p<0.007)$.

Social support from friends moderated the relationship between inactivity temptations and MVPA $(p=0.002)$. The simple slope of the associations between inactivity temptations and MVPA (Figure 2.2) showed that at high levels of social support, inactivity temptations were not associated with MVPA $(\beta=0.001[-0.103,0.105], p<0.987)$. For low levels of social support, inactivity temptations were negatively and significantly associated with MVPA ( $\beta=-0.213[-0.111,-0.415], p<0.0005)$. The association between age and MVPA was moderated by BMI with the negative effect of age increasing relative to BMI ( $\mathrm{p}=0.002$; Figure 3 ). However, the negative associations between 
age and MVPA were significant at both low $(\beta=-0.023[-0.013,-0.033], \mathrm{p}<$ $0.0005)$ and high BMI $(\beta=-0.038[-0.37,-0.039], \mathrm{p}<0.0005)$.

Table 2. 3 Moderating effects of study site age and psychosocial attributes

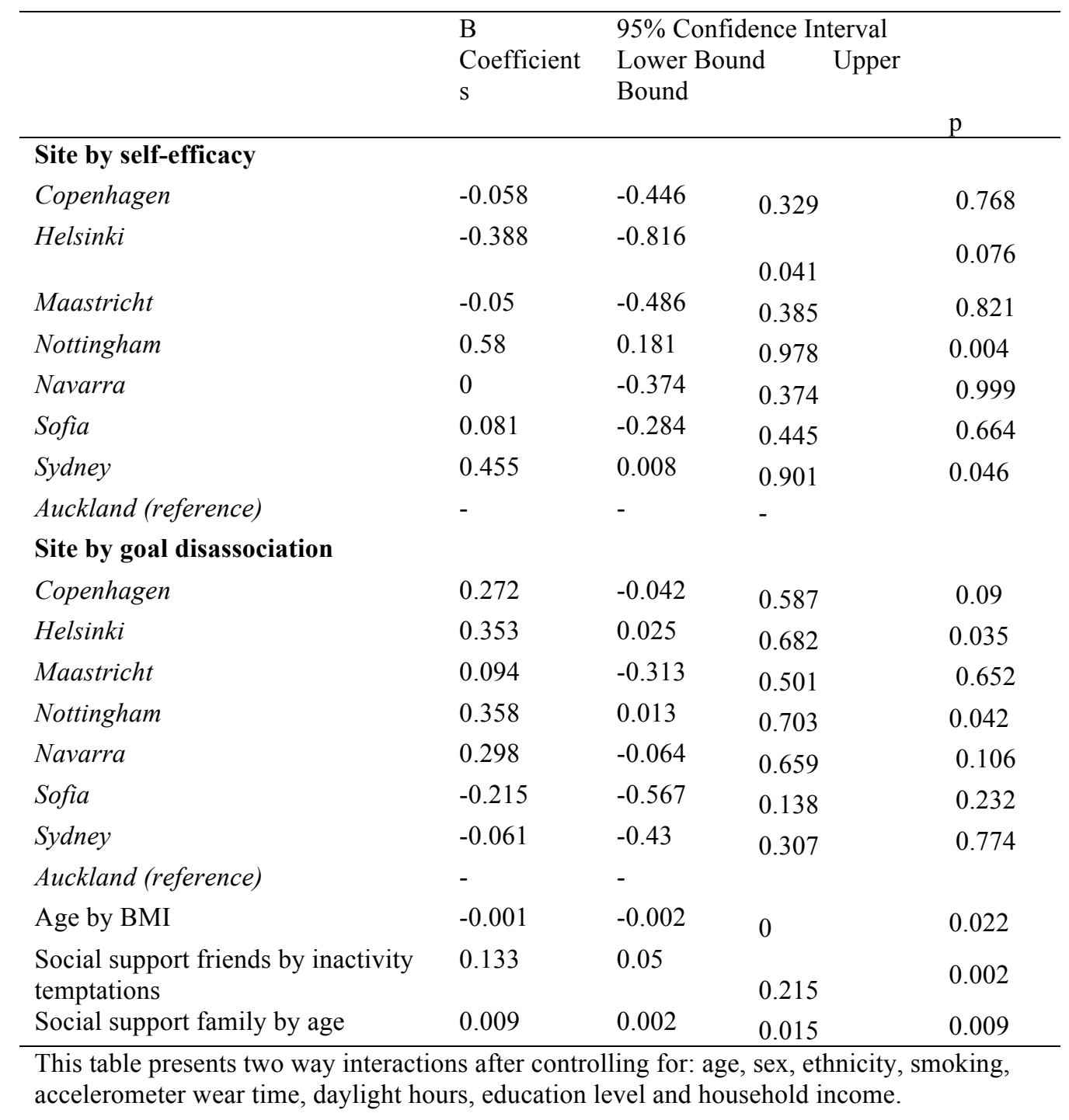


Figure 6. 1 Moderation between social support from family and age

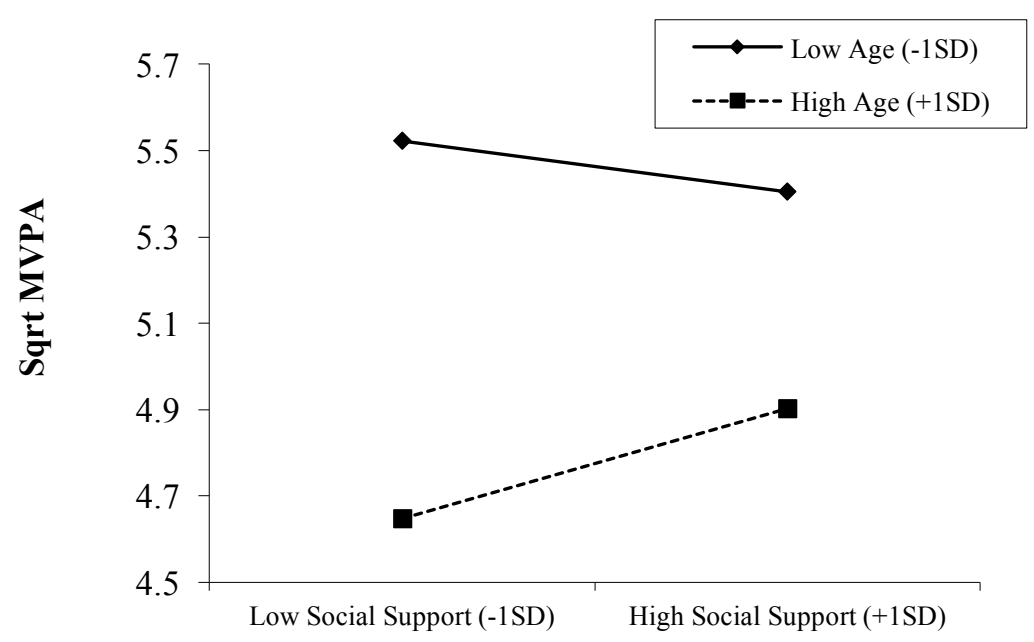

Figure 6. 2 Moderation between social support from friends and inactivity temptations

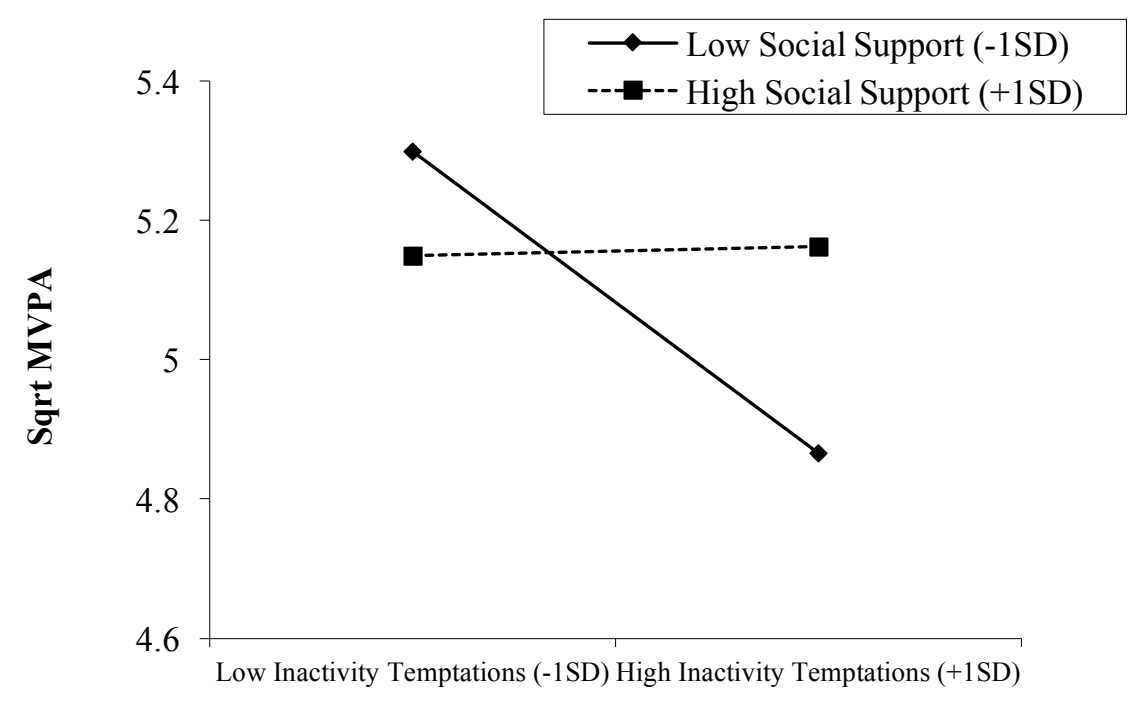




\subsection{Discussion}

This study examined the associations between personal and psychosocial variables and MVPA in adults with pre-diabetes enrolled into the PREVIEW study, while investigating the moderating effects of country, sex and age.

Across all countries, after controlling for covariates, only habit strength of transport behaviours (negative) and intentions toward exercise (positive) were significantly associated with MVPA. Central to the theory of planned behaviour 229 , is the tenet that intention is a key determinant of behaviour. However, intention only predicts a small proportion of behaviour ${ }^{334}$, behaviours also occur as a habitual response to environmental cues without volitional control or awareness ${ }^{335}$. These two constructs (intention and habit strength) have been shown to interact to determine behaviour ${ }^{335}$. While no interaction was found in this study, habit strength was negatively associated with physical activity. Thus, it may be pertinent for interventions to focus on habit strength in combination with intention to affect physical activity behaviours.

Self-efficacy is consistently related to physical activity in the general population 197 and is associated with the adoption and long-term maintenance of physical activity following interventions ${ }^{336}$. However, results in adults at risk of type 2 diabetes are contradictory. Specifically, contrary to findings reported by Donahue et al ${ }^{210}$, self-efficacy was significantly associated with self-reported physical activity in the US Diabetes Prevention Program (USDPP) ${ }^{211}$. In the present study, associations between self-efficacy and MVPA were inconsistent, with significant positive associations only reported in the UK and Australia. Although no obvious explanations for these inter-country differences are apparent, previous studies have postulated that psychosocial correlates of physical activity may be subject to cultural differences ${ }^{202,205}$. In a study run across 4 sites, 2 in the USA, 1 in Belgium and 1 in Australia, Van Dyke and colleagues ${ }^{202}$ found site specific associations between physical activity and psychosocial variables including self-efficacy. Given that the results varied between sites in the same country ( 2 sites in the USA) the authors concluded that some correlates have a more or less important role in differing cultural or environmental contexts. 
A possible explanation for these differences is that psychosocial correlates of physical activity vary according to the physical activity domain (sport, exercise, active transport, occupational, house work) ${ }^{204,337}$. Sternfeld and colleagues ${ }^{337}$ demonstrated that self-efficacy was positively associated with sport and exercise behaviour but not housework or care giving activities. While accelerometers do not provide the context of which domain activity was recroded in, previous studies have shown that leisure time, occupational and active transport behaviours differ by country ${ }^{203}$. It is possible that varying modes of physical activity across different domains (e.g. Cycling for transport in Copenhagen and Maastricht) and their differing underlying determinates, explain these country differences.

Whilst the inter-site differences in self-efficacy and goal adjustment cannot be comprehensively explained, previous research has reported country differences in exercise health beliefs ${ }^{338}$, perceived benefits and barriers to physical activity 339 and the effects of neighbourhood environments on physical activity ${ }^{340}$. Further analysis of such differences is needed and should include cultural and neighbourhood characteristics of the different study sites and varying physical activity domains.

Wrosch and colleagues ${ }^{341}$ have demonstrated that those who are better able to disengage from unattainable goals and reengage with alternative goals experience better physical health. Although this construct is not commonly reported as a correlate of physical activity, previous research in breast cancer survivors suggested that goal adjustment can produce improvements in subjective well-being and somatic health by facilitating greater physical activity and less sedentary time ${ }^{342}$. The present study demonstrates that in Finland and the UK, the ability to disengage from an unobtainable goal is positively associated with time spent in MVPA.

The addition of social support as a component in lifestyle interventions has shown to be important for improving weight loss ${ }^{343}$. However, in a sample of adults with high risk of type 2 diabetes, social support was not predictive of achieving 150 minutes of physical activity ${ }^{210}$. In the present study, the main effects for perceived social support from peers and family were not significantly associated with MVPA. However, peer social support was found to positively moderate the relationship between inactivity temptation and MVPA, showing 
that when social support was low, inactivity temptations were negatively associated with MVPA. However, high peer social support, buffered negative associations between inactivity temptation and MVPA. Similarly, associations between familial social support and MVPA was moderated by age and was only significant among participants aged 1SD above the mean.

These findings suggest that older participants are more reliant on social support from family members to engage in physical activity. While in younger participants, social support from friends helps avert from temptations to be inactive.

There are some limitations to this study. First, it is important to emphasize that this is a cross-sectional study and does not identify causality. Secondly, while a high number of interaction effects were tested in the regression models, the effect of multiple testing was not considered. This could have increased the likelihood of a type I error. Finally, although accelerometers offer more robust assessments of physical activity than self-report ${ }^{179}$, they are not without limitation. Specifically, hip-worn accelerometers capture most movement during locomotion, but cannot account for upper body movement or movement that occurs during activities such as cycling or weight-lifting ${ }^{330}$. Furthermore, the accelerometer is removed during water-based activities and contact sports and may underestimate physical activity. Nonetheless, the 24h, seven-day accelerometer protocol resulted in a greater mean waking wear-time (15.5 hours) than several studies of a similar nature ${ }^{148,280}$. The longer monitoring duration provides greater reliability of average activity estimates.

\subsection{Conclusion}

Only a small number of modifiable factors were associated with MVPA in adults with pre-diabetes. Social support may be of greater importance in older participants and in the presence of high inactivity temptations, while some correlates of MVPA were country-specific (self-efficacy and goal adjustment). Further research is required to explain inter-site differences and these studies should include cultural and neighbourhood characteristics of the different study sites. 


\subsection{Thesis Map}

\begin{tabular}{ll}
\hline Study & Aims
\end{tabular}

1 .

Objectively measured physical activity and sedentary time are associated with cardio-metabolic risk factors in adults with prediabetes: The PREVIEW study.

\section{Findings}

2.

Correlates of physical activity in adults with pre-diabetes from 8 countries: the PREVIEW study
To measure the relationship between
cardio-metabolic risk factors and
objectively measured physical activity and sedentary time.
$\mathrm{H}_{0}$ : The magnitude of the association between physical activity and cardio metabolic risk factors is equal to zero.

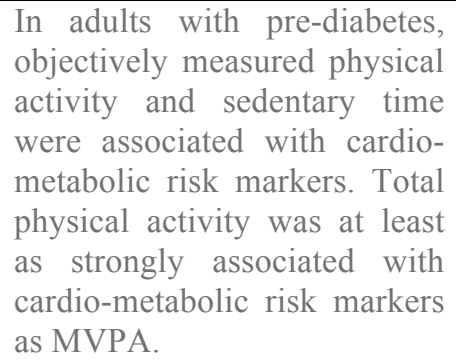
activity and sedentary time were associated with cardiometabolic risk markers. Total physical activity was at least as strongly associated with cardio-metabolic risk markers as MVPA.

Only a small number of
modifiable factors were
associated with MVPA. Social
support may be of more
importance in older
participants and in the
presence of high inactivity
temptations while some
correlates of MVPA were
country-specific (self-efficacy
and goal adjustment).
To assess the correlates of objectively measured physical activity in adults with prediabetes in eight countries and to examine inter-country differences with site-specific interactions.
$\mathrm{H}_{0}$ : The magnitude of association between psychosocial variables and physical activity is equal to zero
$\mathrm{H}_{0}$ : There is no difference between country in the magnitude of association between psychosocial variables and physical activity .

Prevalence of type 2 diabetes in children has increased parallel to childhood obesity. The pathogenic processes that leads to type 2 diabetes and CVD originate in childhood and the severity of the related complications that can ensue is directly related to the duration of exposure. Thus, interventions would be more effective if they were delivered to at-risk populations at the earliest possible age. From study 3 and thereafter, this thesis focused on aspects of child interventions.

3.

Lifestyle behaviours associated with body fat percent in 9-11year-old children, a populationbased, cross-sectional study: The SportsLinx project.
To investigate the associations between body fat percent, and lifestyle behaviours in primary school children on a population level. Further, to examine the consistency of these associations over a 10 -year period.

$\mathrm{H}_{0}$ : The association between lifestyle behaviours and body fat $\%$ is equal to zero $\mathrm{H}_{0}$ : The is no difference between years in the magnitude of association between lifestyle behaviours and body fat $\%$.

4.

The effectiveness of a child weight management intervention; a mixed methods investigation of child and parent's perceptions of the behaviour change process. 


\section{Study 3: Lifestyle behaviours associated with body fat percent in 9-11- year-old children, a population-based, cross-sectional study: The SportsLinx project}

\subsection{Introduction}

Childhood obesity is a significant public health concern, with a third of children in the UK being overweight or obese ${ }^{344}$. Many of the complications and comorbidities associated with excess body fat, such as atherosclerosis and insulin resistance, originate during childhood contributing to the risk of type 2 diabetes, cardiovascular disease and premature death ${ }^{259,260,345,346}$.

Although a constellation of factors contributes to the current childhood obesity epidemic $^{347}$, successful interventions must focus on the modifiable components. Fundamentally, excess body fat is the result of an energy imbalance; an energy intake greater than energy expenditure. Lifestyle factors such as skipping breakfast, screen time, and the consumption of sugar sweetened beverages, sweets and dairy, have been implicated in the development of obesity in children ${ }^{265-267}$. However, results from large population studies remain inconsistent ${ }^{35,36}$. Indeed, in a study of 137,593 youth (10-16 years), from 34 countries found that low physical activity levels and television viewing were associated with obesity, but no association was found for the intake of fruit, vegetables or soft drinks or the time spent using computers. Surprisingly, a negative association was found between the frequency of the consumption of sweets (candy in the USA) and obesity ${ }^{36}$. Furthermore, the ISCOLE study of 6,025 children (9-12 years) from 12 countries, failed to find an association between dietary patterns and obesity 35, in accord with population-level studies in the UK ${ }^{268,269}$. However, methodological limitations, such as the pooling of responses to a food frequency questionnaire to produce a "healthy" and "unhealthy" score ${ }^{268,269}$, precludes further interpretation of these findings. Given the counter intuitive and inconsistent associations between obesity and specific foods, defining foods as "healthy" and "unhealthy" in the context of obesity and weight gain may not be appropriate. For example, cross-sectional and prospective studies have shown full fat milk and dairy fat to be negatively associated with obesity and weight gain ${ }^{138,270}$. Yet, full fat milk has been classed as "unhealthy", while skimmed varieties have been classified as "healthy" for assessing associations with 
obesity $^{268,269}$. Consequently, creating a composite score may mask associations between specific foods and obesity.

There is evidence that screen-based behaviours have changed across Europe over the past 15 years. Specifically, TV viewing has decreased, while computer use for gaming and non-gaming purposes has sharply increased ${ }^{271}$. Likewise, changes in dietary behaviours have been reported over the last decade, whereby the UK National Food Survey shows there has been a year-on-year shift from full fat to skimmed milk varieties and an increase of wholemeal bread ${ }^{272}$. Previous analysis of the SportLinx cohort show improvements in the consumption of fruit, vegetables, and salad ${ }^{273}$. In the UK, there have also been changes in the composition of foods. In 2008, 55 percent of soft drinks consumed by UK children contained no added sugar compared to 46 percent in $2004^{274}$. Despite these changes, it is unclear whether lifestyle behaviours and food choices are associated with body fat and whether these associations have changed over time. To my knowledge, no study has investigated changes in the associations between these lifestyle behaviours and body fat percent.

The aim of this study was therefore to investigate the associations between body fat percent and the consumption of specific foods, dietary and physical activity behaviours in primary school children. Further, we aimed to examine the consistency of these associations over a 10-year period.

\subsection{Methods}

A complete description of the methods used in the SportsLinx study is provided in chapter 4.

\subsubsection{Participants and Settings}

The SportsLinx program has been described in more detail elsewhere $273,305,308$. Briefly, SportsLinx was a serial, cross-sectional physical activity, nutrition and health program conducted in primary schools within the Liverpool Local Education Authority (UK). All year 5 pupils (9-10 years) were invited to take part annually, pending informed parental consent and participant assent. Between 2004 and 2013 nearly 16,000 $(n=15,977)$ children completed an anthropometric assessment and lifestyle survey ${ }^{273,348}$ and are included in the analysis. 


\subsubsection{Statistical analyses}

Descriptive statistics, independent samples t-test, and Pearson's chi squared tests were used to examine differences in the basic characteristics and questionnaire responses between sexes using the statistical package IBM SPSS Statistics for Mackintosh, version 22.0 (IBM Corp., Armonk, NY, USA).

As data were collected from schools across Liverpool, multi-level models were adopted to account for the nested nature of the data ${ }^{349}$. A two level multi-level model was constructed to examine associations between lifestyle survey responses and body fat percent. Participants were defined as the first level and school as the second level. Data were analysed using MLwiN v.3.01 software (Centre for Multilevel Modelling, University of Bristol, UK). The model was constructed in three stages. Firstly, a backwards stepwise deletion approach was used to identify all predictor variables that were significantly associated with the outcome variable. Regression coefficients were assessed for significance using the Wald statistic and a Chi-square distribution. Secondly, all significant predictor variables were entered into a fully adjusted model with known confounders (sex, year, MSFT and SES). Finally, two-way interactions were explored to establish whether predictors differed by sex or year. Alpha was set at $\mathrm{p}<.05$ for main effects and $\mathrm{p}<0.1$ for interaction terms ${ }^{350}$.

Multiple imputation was used to address the potential bias and loss of precision that can result from complete-case analysis. REALCOM-IMPUTE software with MLwiN ${ }^{351}$ was used to generate 10 imputed datasets with missing values replaced by plausible values based on known covariates and distributional information. A burn in of 1,500 iterations was used before the first imputation, followed by imputations after every $500^{\text {th }}$ iteration, resulting in a total of 6,500 iterations. The model of interest was fitted to each imputed dataset and the results were combined using Rubin's rule ${ }^{352}$.

\subsection{Results}

Characteristics of children from the 116 schools that completed the SportLinx questionnaire and skinfold assessments between 2004 and 2013 are presented in Table 3.1. Girls $(n=7,949)$ had a greater prevalence of overweight and obesity than boys $(n=7,955 ; \mathrm{p}<0.001)$ and a higher percent body fat $(\mathrm{p}<0.001)$. Responses to the lifestyle survey are presented in the supplementary materials 
(tables S1-S4). Significant sex differences were present in dietary behaviours, activity behaviours, screen-based activities and food intake.

Table 3. 1 Participant characteristics SportsLinx

\begin{tabular}{|c|c|c|c|c|c|}
\hline & \multicolumn{2}{|c|}{ Boys $(\mathrm{n}=7,955)$} & \multicolumn{2}{|c|}{ Girls $(n=7,949)$} & $\mathrm{P}$-value \\
\hline Age (years) & 9.7 & $(0.4)$ & 9.7 & $(0.4)$ & 0.84 \\
\hline Height (cm) & 137.8 & $(6.2)$ & 137.5 & $(7.8)$ & 0.001 \\
\hline Weight (kg) & 39.2 & $(13.9)$ & 39.4 & $(13.7)$ & 0.223 \\
\hline $\operatorname{BMI}\left(\mathrm{kg} \cdot \mathrm{m}^{-2}\right)$ & 20.4 & $(6.9)$ & 20.7 & $(6.8)$ & 0.024 \\
\hline BMI z-score ${ }^{a}$ & 0.8 & $(1.5)$ & 0.9 & $(1.4)$ & 0.004 \\
\hline Fat percent & 23.8 & $(8.9)$ & 27.2 & $(8.6)$ & 0.000 \\
\hline $\begin{array}{l}\text { Prevalence of overweight } \\
{ }^{b}(\%)\end{array}$ & 17.7 & & 21.0 & & 0.000 \\
\hline Prevalence of obese ${ }^{b}(\%)$ & 11.4 & & 13.5 & & 0.000 \\
\hline
\end{tabular}

Data are means and standard deviations unless stated otherwise. ${ }^{a}$ BMI Z-score denotes the standard deviation score calculated from International Obesity Task Force values. ${ }^{\mathrm{b}}$ Overweight and obesity were defined by using the International Obesity Task Force criteria.

Results of the multilevel models are presented in Table 3.2. The estimated variance at the school level, indicated by the null model, suggests a significant inter-individual difference across schools in body fat percent $\left(\chi^{2}=2,141 \mathrm{p}<\right.$ 0.001). The interclass correlation coefficient revealed that $16.4 \%$ of the variability in body fat percent was explained by the school-level effect. Therefore, $83.6 \%$ was accounted for by individual level factors.

The crude model which contained all lifestyle behaviours that were significantly associated with body fat percent explained approximately $8.6 \%$ of the variance in body fat. The adjusted model, which controlled for sex, fitness, IMD score and year, accounted for $15.3 \%$ of the variance. Associations between bed time, active transport, sugar sweetened cereals, sugar sweetened beverage, low calorie beverage and full fat milk were not attenuated after adjustment for confounding variables. The final model with interaction terms explained $15.5 \%$ of the total variation in body fat.

After adjusting for IMD score, sex, fitness and year, significant positive associations were observed between bed time and the dependent variable body fat percent (Table 3.2). All bed times later than $8 \mathrm{pm}$ (the reference category) 
were positively associated with body fat percent. The use of an active mode of transport to get to school was negatively associated with body fat percent. Reporting the intake of full fat milk was negatively associated body fat. Counterintuitively, sugar sweetened cereal and sugar sweetened beverage intake were negatively associated body fat percent. Low calorie beverages were positively associated with body fat percent. Two way interactions indicate that the positive association between low calorie beverages and body fat percent is moderated by sex, with a negative association for girls.

\subsection{Discussion}

The aim of this study was to investigate the associations between body fat percent and lifestyle behaviours in primary school children on a population level. Further, the aim was to examine the consistency of these associations over a 10 year period.

To date, interventions aimed at reducing overweight and obesity and maintaining this change have had limited success ${ }^{275}$. Understanding the role of specific types of foods and lifestyle behaviours in childhood overweight and obesity are of key importance for the development of successful lifestyle interventions. This study shows that in a population of 15,977 children aged 9-11 years in Liverpool (UK), lifestyle behaviours including bed time, full fat milk consumption, active transport to school, soft drink choice and sugar-sweetened cereals explained $8.6 \%$ of the variation in body fat percent and remained significant after adjustment for confounding variables, sex, fitness, IMD score and year. A novel finding of this study is that these associations remained consistent over a 10-year period. To my knowledge, this is the first study to examine change in associations between lifestyle behaviour and body fat over time which suggests that despite alterations in behaviour and the composition of foods, associations with body fat have not significantly changed over a 10 -year period.

Models revealed that going to bed later than $8 \mathrm{pm}$ was positively associated with body fat percent. This is consistent with two systematic reviews that have shown short sleep and late bed times are associated with an increased risk of being overweight or obese ${ }^{353,354}$. Insufficient sleep has been shown to affect dietary patterns, resulting in food cravings and a greater consumption of energy dense foods ${ }^{355}$. Further explanations for this association may be that sleep is 
negatively impacted by sedentary pursuits, such as television watching ${ }^{356}$, and positively affected by exercise ${ }^{357}$. Although previous studies have shown that television watching is associated with both reduced sleep and increased BMI, no association was found between television watching and body fat in the present study nor was there an interaction between television watching and bed time. This may be due to a lack of sensitivity in the dichotomous variables used to monitor television watching. Although this cross-sectional study cannot determine a causal direction of the association, bed time and sleep duration are important considerations when planning lifestyle interventions for the prevention of obesity.

Using an active mode of transport to get to school (i.e., walking, cycling, or by scooter) was negatively associated with body fat percent. A systematic review of 13 studies examining actively commuting to school and body weight found that active commuters accumulate significantly more physical activity and expend more calories than their non-active commuting counterparts ${ }^{358}$. Taken together, these findings suggest that commuting to school may offer an effective approach to increase daily activity and reduce body fat by increasing daily energy expenditure. This is the first population-level study to demonstrate that active modes of school travel are negatively associated with body fat percent. The National Travel Survey has indicated that the use of active transport to school is declining, due to increasing commuting distances ${ }^{359}$. We found no significant change in the association between body fat percent and active transport to school over the 10-year period. Policies designed to affect a population-level shift to more active modes of travelling to school may offer a major opportunity for public health improvement. 
Table 3. 2 Multi level model of associations between lifestyle behaviours and body fat percentag

\begin{tabular}{|c|c|c|c|c|c|c|c|c|}
\hline \multirow{3}{*}{ Predictor variables } & \multicolumn{4}{|c|}{ Crude model (model 1) } & \multicolumn{4}{|c|}{ Adjusted model (model 2) } \\
\hline & \multirow[t]{2}{*}{ Beta } & \multicolumn{2}{|c|}{$95 \% \mathrm{CI}$} & \multirow[t]{2}{*}{ p-value } & \multirow[t]{2}{*}{ Beta } & \multicolumn{2}{|c|}{$95 \% \mathrm{CI}$} & \multirow[t]{2}{*}{$\mathrm{p}$-value } \\
\hline & & Low & High & & & Low & High & \\
\hline Constant & 27.025 & 26.44 & 27.61 & $<0.0005$ & 25.96 & 24.87 & 27.05 & $<0.00$ \\
\hline Sugar sweetened cereals & -0.70 & -1.02 & -0.37 & $<0.0005$ & -0.62 & -0.94 & -0.29 & $<0.000$ \\
\hline Ordinary sausages & -0.52 & -0.90 & -0.14 & 0.007 & -0.28 & -0.66 & 0.11 & 0.1 \\
\hline Full fat milk & -1.10 & -1.41 & -0.79 & $<0.0005$ & -0.97 & -1.27 & -0.67 & $<0.00$ \\
\hline Sugar sweetened beverages & -0.37 & -0.66 & -0.08 & 0.013 & -0.39 & -0.68 & -0.10 & 0.00 \\
\hline Low calorie beverages & 0.54 & 0.16 & 0.91 & 0.005 & 0.54 & 0.19 & 0.89 & 0.0 \\
\hline Active Transport & -1.34 & -1.71 & -0.97 & $<0.0005$ & -1.00 & -1.37 & -0.62 & $<0.00$ \\
\hline Week Sport & -0.50 & -0.78 & -0.22 & $<0.0005$ & -0.13 & -0.41 & 0.14 & 0.33 \\
\hline \multicolumn{9}{|c|}{ Usual bed time during the week $\leq 8: 00 \mathrm{pm}($ Ref) } \\
\hline 8:00-8:59pm & 1.66 & 1.15 & 2.18 & $<0.0005$ & 1.59 & 1.08 & 2.10 & $<0.000$ \\
\hline 9:00-10:00pm & 1.09 & 0.62 & 1.56 & $<0.0005$ & 1.04 & 0.57 & 1.50 & $<0.000$ \\
\hline$\geq 10: 00 \mathrm{pm}$ & 0.92 & 0.33 & 1.50 & 0.002 & 1.18 & 0.60 & 1.76 & $<0.000$ \\
\hline High fibre cereals & -0.31 & -0.66 & 0.03 & 0.071 & -0.19 & -0.52 & 0.15 & 0.28 \\
\hline Computer on the weekend & -0.74 & -1.02 & -0.46 & $<0.0005$ & 0.18 & -0.12 & 0.47 & 0.2 \\
\hline IMD Score & & & & & 0.01 & 0.00 & 0.02 & 0.00 \\
\hline MSFT & & & & & -0.04 & -0.04 & -0.03 & $<0.00$ \\
\hline Sex (girl) & & & & & 3.06 & 2.78 & 3.35 & $<0.000$ \\
\hline
\end{tabular}


Table 3.2 continued

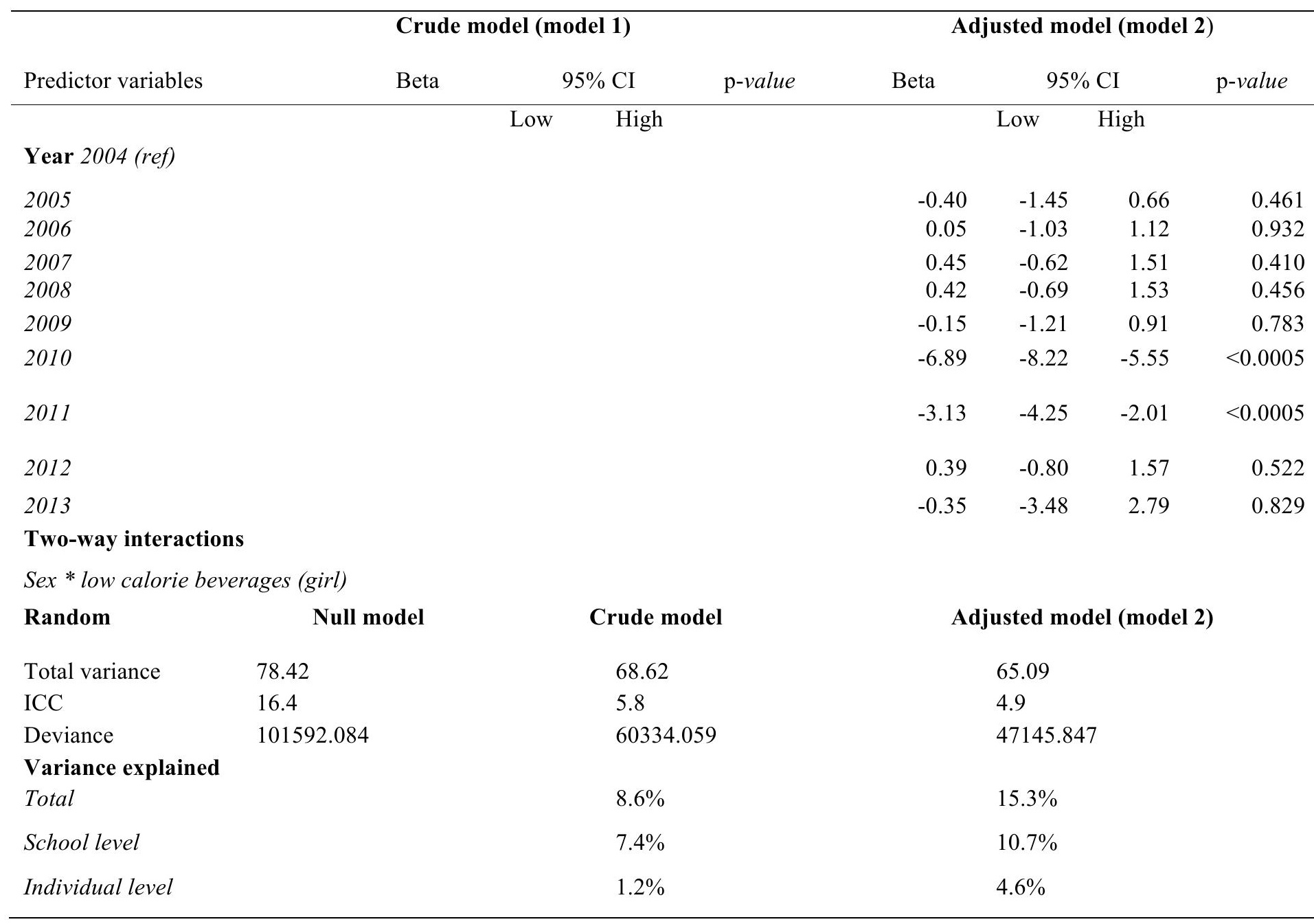


The Scientific Advisory Committee on Nutrition has recommended the average intake of free sugars across the UK population should not exceed $5 \%$ of total energy intake. This guideline is supported by a review which showed that the intake of free sugars and sugar-sweetened beverages were a determinant of body weight by modifying total energy intake ${ }^{267}$. According to the national dietary survey (2012), this recommendation is exceeded by the general population, and especially by children. Soft drinks and fruit juice, followed by cereals and cereal products (mainly cakes, biscuits and breakfast cereals), are the leading sources of free sugars ${ }^{304}$. In the present study, no association was found between reporting the intake of biscuits, cakes, chocolate bars, fizzy drinks or added sugar, but a counterintuitive negative association was found between body fat percent and sugar-sweetened cereals and sweetened beverages (i.e., orange squash, Ribena, Vimto). This is not the first study to report counterintuitive association for foods high in sugar ${ }^{36}$. It is important to note that the current survey does not quantify portion size or total energy intake, but is an indicator of dietary choices on a population level. It is possible that overweight children consumed sugar-sweetened cereals and sweetened soft drinks more frequently or in greater portion sizes. Another possible explanation is that overweight children are more likely to under-report unhealthy food intake than their normal weight counterparts ${ }^{360}$. We found that the consumption of low calorie soft drinks was positively and negatively associated with body fat in boys girls respectively. This surprising outcome, for boys at least, supports previous findings that diet soft drinks are positively associated with body weight, suggesting that calorie content is not solely responsible for this association ${ }^{361}$. In adults, the San Antonio Heart Study found significant positive dose-response relationship between low calorie beverages and BMI, from baseline to a 7-9 year follow-up 362. A possible explanation may be that soft drinks, regardless of their sugar content, are regularly consumed alongside high-energy snacks or during sedentary behaviours, such as television watching. Conversely, soft drinks, including sports drinks, are frequently sold at sports clubs and centres and may be consumed while also participating in physical activity ${ }^{363}$. Although we cannot determine the direction of this association given the cross-sectional nature of the study, it is possible that children who become overweight or obese 
may consume diet soft drinks in an attempt to reduce their calorie intake. Reverse causality has previously been suggested as a possible explanation for the positive associations between the consumption of low calorie soft drinks and obesity ${ }^{364}$. However, prospective studies have shown that these associations remain significant after controlling for BMI at baseline ${ }^{365}$ and while analysing normal weight adults separately ${ }^{362}$. Therefore, it is unlikely that reverse causality can explain this association. Other studies have suggested a cognitive process, whereby being informed of the low-calorie status of a "diet" or reduced energy food may lead to overcompensation in the intake of other foods rationalized by the previous saving ${ }^{366}$. Alternatively, animal studies have pointed to a learnt effect whereby the consumption of low-energy sweeteners leads to a disruption of sensory cues that induce conditioned hormonal, neural, and metabolic responses, which promote the effective utilization of energy by anticipating and preparing for the expected nutrients in the gastrointestinal tract. Repeatedly consuming sweet tasting foods with no energy content may lead to the failure to anticipate calories from sugar when they are consumed, disrupting the signals that lead to satiety ${ }^{367}$. Regardless of the underlying mechanisms, our findings demonstrated that the association between low-calorie beverages and body fat differs between boys and girls. Very few studies have distinguished between regular and sugar-free or diet drinks when investigating association with body fat or obesity; this is the first study to show a sex difference in the associations with body fat in a large population over time. Further research is required to establish the wider context in which diet and low calorie soft drinks are related to body fat. Based on our findings, future studies should consider different circumstances for boys and girls.

The national diet and nutrition survey has shown breakfast cereals to be a major contributor to free sugar intake among UK children, while free sugars have also been implicated with encouraging over-eating and weight gain ${ }^{267}$. Despite this proposed link, an unexpected, negative association was found between body fat percent and sugar sweetened cereals. This finding agrees with outcomes from the NHANES study which found that regardless of sugar content, children who ate cereal had significantly lower BMI compared with children who did not. Furthermore, cereal consumption was associated with lower fat intake and a higher intake of carbohydrates, whole grains, and a range of vitamins ${ }^{368}$. 
Similarly, in a recent study of Australian children, no associations were found between anthropometric measures and the choice of non-sweetened or sweetened breakfast cereal. Both added and free sugar intake was similar between non-cereal consumers and cereal consumers, while cereal consumers had lower sodium and higher intake of total sugars, carbohydrate, dietary fibre, and almost all other micronutrients with no difference between non-sweetened or sweetened breakfast cereal consumers ${ }^{369}$. These findings might be explained by a lower Glycaemic index (GI) diet in participants who consumed breakfast cereals due to the increased fibre content. Glycaemic Index has been shown to be an important factor in weight management preventing weight regain after initial weight loss ${ }^{119}$ and improving weight loss ${ }^{370}$. The energy density and GI of breakfast cereals is not solely dependent on sugar content but also processing techniques and the quantity and type of fibre ${ }^{371,372}$. Research has shown higher dietary fibre intake in participants consuming breakfast cereals regardless of sugar content ${ }^{368,369}$.

We found that consumption of full fat milk was negatively associated with body fat, although no association was found between low fat milk varieties. Previous studies have provided inconsistent reports of the relationship between milk/dairy intake in children and adiposity. Several studies have suggested a negative association ${ }^{373,374}$, while others found no association ${ }^{266}$ or positive associations 375. Several studies have shown that soft drink consumption displaces milk consumption in children and adolescents ${ }^{376,377}$, suggesting that the children with a high intake of milk also consume fewer soft drinks. However, in the present study associations between soft drink consumption and body fat were inconclusive and could not explain the lack of association for low fat milk. Few studies have distinguished between low and full fat dairy products in children in relation to body fat; Berkey et al. ${ }^{375}$ reported greater weight gain among children who drank the most milk. However, while the consumption of skimmed milk was associated with weight gain, dairy fat was not, suggesting it is not the fat content that is responsible for the weight gain ${ }^{375}$. In a cohort of children up to 3 years of age, a higher milk fat consumption was associated with lower odds of obesity ${ }^{378}$. In adults, a systematic review found that high-fat dairy consumption was inversely associated with obesity risk ${ }^{138}$. Similarly, a prospective study in Swedish adults showed the intake of whole-fat milk was 
associated with lower rates of central obesity over a 12 -year period ${ }^{270}$. It is important to acknowledge that the cross-sectional nature of this study cannot rule out reverse causality as an explanation for the observed associations. Given current dietary guidelines, overweight and obese children may have opted for low fat dairy options in an effort to prevent further weight gain. However, a number of prospective studies that control for BMI at baseline have found similar results $266,379,380$. This suggests that reverse causality cannot completely explain these associations. Possible mechanisms for the inverse association between dairy fat and obesity are unclear but may include bioactive effects of the fatty acids contained in milk which have been shown to have an effect on low grade inflammation, oxidative stress ${ }^{381}$ and insulin resistance ${ }^{138}$. Furthermore, the greater fat content may increase satiety and result in a net reduced calorie intake ${ }^{382}$. Nonetheless, nutritional guidelines continue to recommend reduced fat dairy products ${ }^{383}$. The rationale for these guidelines are twofold; first, to reduce energy density which may contribute to a positive energy balance and fat gain and, second, dietary cholesterol and saturated fatty acids raise serum cholesterol concentrations, and the ratio of low-density lipoprotein to high-density lipoprotein cholesterol ${ }^{384}$. It is thought that reducing the intake of cholesterol and saturated fatty acids will lower the risk of cardiovascular disease ${ }^{385}$. However, a recent meta-analysis concluded that the consumption of saturated fat was not associated with the risk of cardiovascular disease ${ }^{386}$. Further, two recent reviews have shown that dairy products and milk fat do not increase risk markers of cardiovascular disease ${ }^{138,387}$. Existing evidence from prospective studies has suggested that despite its energy and saturated fat content, full fat milk may be protective of weight gain and obesity 138,270. The findings of the present study add to this evidence by showing that at a population level, the consumption of full fat milk is negatively associated with body fat percent in 9-11 years old children.

Body fat percent is unfavourably associated with cardio-metabolic risk markers in children, including fasting triglycerides, HDL-C, fasting glucose, insulin resistance, and CRP ${ }^{388,389}$. In the present study, using an active mode of transport to school and choosing full fat milk was associated with a $\sim 1 \%$ lower body fat $\%$. The clinical relevance of this finding is difficult to deduce given that the relevance of excess adipose tissue is dependent on the region of the body in 
which it is stored and the weight status of the individual ${ }^{389}$. To our knowledge, no studies have reported the clinical relevance of reduction in body fat $\%$ in children. However, several studies have suggested that even small reductions in BMI $>0.25 \mathrm{SD}^{390}$ and $>0.5 \mathrm{SD}^{391}$ are associated with significant improvements in triglycerides, LDL-C, CRP and insulin sensitivity.

This study has several strengths. First, the large sample size of 15,977 participants and the duration of the study spanning 10 years has allowed us to test consistency of associations between body fat and lifestyle behaviours over time. To my knowledge, this is the first study of this kind to examine a complete cross-section of children (aged 9-11 years) from a UK city. Secondly, the pathogenic processes associated with obesity are related to body fat. BMI is widely used in population studies as an estimate of adiposity because it is relatively quick to measure, cheap and non-invasive. However, BMI is a composite measure using height and weight, and is not a measure of body composition. Skinfold measures allow a 2-compartment model to be determined: fat mass and fat free mas. To my knowledge, this is the first study of this kind to use skinfold measures on a population of this size. The precision of this measure is enhanced by using the same ISAK qualified anthropometrist over the 10-year study removing the potential for inter examiner error. Finally, the use of multilevel analyses and multiple imputation techniques add to the rigour of our methodology by accounting for clustering between schools and by handling missing data respectively.

There are also several limitations that warrant attention. The cross-sectional study design does not allow us to draw conclusions about a causal relationship between dietary factors and body fat. Further, the lifestyle behaviours were captured with a self-report questionnaire which may be susceptible to social desirability and recall bias ${ }^{392}$. The SportsLinx lifestyle survey was designed to identify specific foods consumed on the previous day as an indicator of food choice and lifestyle behaviours at a population level. The survey did not allow frequency or portion size to be quantified and therefore energy nutrient intake is unknown.

Previous studies investigating associations between lifestyle behaviours and body fat or BMI have shown negative associations between physical activity and 
markers of obesity, ${ }^{35,268,269}$. In the present study, we did not have a direct measure for physical activity, which may have affected our results. In the absence of a measure of physical activity, the present study controlled for aerobic fitness. Although we recognize that aerobic fitness is partially genetic in origin, it can also be a reflection of recent physical activity levels ${ }^{393}$ as physically active children have higher cardiorespiratory fitness than their inactive counterparts ${ }^{394}$. Furthermore the MSFT is predictive of cardiometabolic health irrespective of BMI ${ }^{395}$.

\subsection{Conclusions}

This study found that lifestyle behaviours are associated with body fat percent in a large population of children aged 9-11 years with no significant change in these associations over a 10-year period. Policies and interventions targeting population shifts in behaviours, such as active transport to school, sleep time and consumption of full fat milk may offer a major opportunity for public health improvement. Further research is required to establish the wider context in which low calorie soft drinks are linked with body fat and future studies should consider different mechanisms for boys and girls. 


\subsection{Thesis Map}

\begin{tabular}{|c|c|}
\hline Study & Aims \\
\hline $\begin{array}{l}\text { Objectively measured physical } \\
\text { activity and sedentary time are } \\
\text { associated with cardio- } \\
\text { metabolic risk factors in adults } \\
\text { with pre-diabetes: The } \\
\text { PREVIEW study. }\end{array}$ & $\begin{array}{l}\text { To measure the relationship between } \\
\text { cardio-metabolic risk factors and } \\
\text { objectively measured physical activity and } \\
\text { sedentary time. } \\
\mathrm{H}_{0} \text { : The magnitude of the association } \\
\text { between physical activity and cardio } \\
\text { metabolic risk factors is equal to zero. }\end{array}$ \\
\hline $\begin{array}{l}2 . \\
\text { Correlates of physical activity in } \\
\text { adults with pre-diabetes from } 8 \\
\text { countries: the PREVIEW study. }\end{array}$ & $\begin{array}{l}\text { To assess the correlates of objectively } \\
\text { measured physical activity in adults with } \\
\text { prediabetes in eight countries and to } \\
\text { examine inter-country differences with } \\
\text { site-specific interactions. } \\
\mathrm{H}_{0} \text { : The magnitude of association between } \\
\text { psychosocial variables and physical } \\
\text { activity is equal to zero } \\
\mathrm{H}_{0} \text { : There is no difference between country } \\
\text { in the magnitude of association between } \\
\text { psychosocial variables and physical } \\
\text { activity. }\end{array}$ \\
\hline
\end{tabular}

Findings

3.
Lifestyle behaviours associated
with body fat percent in 9-11-
year-old children, a population-
based, cross-sectional study:
The SportsLinx project.

To investigate the associations between body fat percent, and lifestyle behaviours in primary school children on a population level. Further, to examine the consistency of these associations over a 10 -year period

$\mathrm{H}_{0}$ : The association between lifestyle behaviours and body fat $\%$ is equal to zero

$\mathrm{H}_{0}$ : There is no difference between years in the magnitude of association between lifestyle behaviours and body fat $\%$.

\section{In adults with pre-diabetes, objectively measured physical activity and sedentary time were associated with cardio- metabolic risk markers. Total physical activity was at least as strongly associated with cardio-metabolic risk markers as MVPA.

Only a small number of
modifiable factors were
associated with MVPA.
Social support may be of
more importance in older
participants and in the
presence of high inactivity
temptations while some
correlates of MVPA were
country-specific (self-efficacy
and goal adjustment).

No significant change in associations between behaviours and body fat were found over a 10-year period. Active transport to school, sleep and consumption of full fat milk were negatively associated with body fat.

\begin{abstract}
4.
The effectiveness of a child weight management intervention; a mixed methods investigation of child and parent's perceptions of the behaviour change process.

To report on the effectiveness of a weight management intervention aimed at producing sustained change in diet and physical activity behaviours and reduction of type 2 diabetes risk factors. To give the contextual, subjective experience of the participants and their parents on the theoretical framework of an intervention and how this may affect compliance with the program objectives.
\end{abstract}


8 Study 4 :The effectiveness of a child weight management intervention; a mixed methods investigation of child and parent's perceptions of the behaviour change process

\subsection{Background}

Many of the complications and comorbidities associated with overweight and obesity originate during childhood. Obesity is the prominent risk factor for cardiovascular disease (CVD) and type 2 diabetes, two of the leading causes of death globally ${ }^{76,396}$. The underlying cause of CVD, atherosclerosis, develops during childhood and is positively associated with BMI ${ }^{259}$. Consequently, BMI during childhood is predictive of CVD in adults ${ }^{260}$. Similarly, type 2 diabetes is a progressive disorder that begins with insulin resistance ${ }^{345}$. Impaired glucose tolerance is fairly common occurring in $15 \%$ to $25 \%$ of obese children ${ }^{69,70}$. Although not all children with IGT or impaired fasting glucose (IFG) develop type 2 diabetes, the rate of conversion from IGT/IFG to type 2 diabetes appears to occur faster in children than adults, in as little as $12-21$ months ${ }^{72}$. A prospective study that followed 117 obese adolescents for two years showed that $8 \%$ of those with IGT progressed to type 2 diabetes, $45.5 \%$ returned to normal glucose tolerance and 30.3\% remained IGT. Severity of obesity, higher baseline IGT and ethnicity were predictive of progression to type 2 diabetes, while a reduction in BMI predicted a return to normal glucose tolerance ${ }^{72}$. In a similar study that followed 128 white, obese children and adolescents, $75 \%$ returned to normal glucose tolerance, $16 \%$ remained IGT and 2\% developed type 2 diabetes, with higher baseline 2-h glucose and weight gain predicting the development of type 2 diabetes. Similarly, Jaruratanasirikul et al. ${ }^{73}$, followed 177 obese children and adolescents for 3 to 6 years. During follow-up, 14.4\% developed type 2 diabetes and weight gain, IGT, BMI, FPG and HOMA-IR were predictive of developing type 2 diabetes ${ }^{73}$. Therefore, while many children with IGT/IFG return to normal glucose tolerance, progression from IGT/IFG to type 2 diabetes is closely related to obesity and weight gain. Consequently, the growing prevalence of childhood obesity is paralleled by a rise in type 2 diabetes among children and adolescents ${ }^{8-10}$. In a retrospective cohort study of 369,362 children from the UK aged 2 to 15 years, the annual incidence of type 2 diabetes per 
100,000 persons increased from 6.4 between 1994 and 1998 to 33.2 between 2009 and 2013, with obese individuals showing greatest risk of incident ${ }^{397}$.

The full public health burden of developing type 2 diabetes during childhood may not yet have been realised. Adults with early onset type 2 diabetes (diagnosed between 18-44yrs) experience more aggressive complications than usual onset type 2 diabetes (diagnosed $>45 \mathrm{yrs}$ ); they are more likely to start insulin therapy, suffer from microalbuminuria and experience macrovascular complications such as myocardial infarction ${ }^{11}$. Long term follow up studies in children are lacking, however, a lifetime exposure to hyperglycaemia may cause a higher risk of complications over time. Predictive models by Rhodes et al. ${ }^{85}$ suggest adolescents with type 2 diabetes may lose approximately 15 years from average remaining life expectancy and may experience severe, chronic complications of type 2 diabetes by their $40 \mathrm{~s}{ }^{85}$.

There is a compelling argument to start interventions during childhood to prevent or delay the onset of type 2 diabetes. The US diabetes prevention program ${ }^{246}$, The Finish Diabetes Prevention program ${ }^{21}$ and the Chinese Da Qing study ${ }^{398}$ provide strong evidence that lifestyle interventions, producing moderate weight loss, through dietary changes and increased physical activity, can prevent or delay the onset of type 2 diabetes in adults. Clinical trials in children are lacking for the understandable reason that very long follow-ups would be required to track the progression of type 2 diabetes over a lifetime. However, a series of systematic reviews and meta-analyses in obese children and adolescents offer encouragement that interventions can produce improvements in BMI and cardio-metabolic risk factors through changes to dietary, physical activity and sedentary behaviour, at least in the short to medium term ${ }^{31,32,263,264}$. It is well known that sustainable behaviour change is difficult and many participants are not successful or experience weight regain ${ }^{275}$. A fundamental component to successful interventions is the inclusion of a psychological behaviour modification framework ${ }^{32}$. However, the behaviour change process is demanding, invasive and personal, with many of the strategies developed for use with adults and it is not known if these strategies are equally effective in children. Furthermore, individual factors that determine failure or success may not be captured with clinical measures. 
The aim of this process evaluation was to report the child and parent perspectives on the effectiveness of a critical research informed weight management intervention aimed at producing sustained change in diet and physical activity behaviours and reduction of type 2 diabetes risk factors. This study aims to give the contextual, subjective experience of the participants and their parents allowing future early intervention studies to better understand how participants may interpret the theoretical framework of an intervention and how this may affect compliance with the program objectives.

\subsection{Recruitment}

If any intervention is to be effective it must be accessible to its target audience and achieve good uptake and retention. However, very few studies in overweight and obese children provide details of their recruitment strategies or their effectiveness.

The negative stigma associated with being overweight or obese has been identified as a barrier to participation ${ }^{399}$. This highlights the importance of not labelling participants as overweight or obese and focusing the intervention on a healthy lifestyle as opposed to weight management. Recruitment strategies identified by other studies include the use of flyers and posters in public places, local media and school newsletters ${ }^{400} 401$. Morrison and colleagues ${ }^{400}$ identified the importance of involving an adult trusted by the parents and child to facilitate recruitment, such as a doctor, teacher, or community professional.

The target audience for this intervention was identified by the Swanlinx health and wellbeing program. Swanlinx was set up in Swansea as a "sister" program to the Sportslinx program described in chapter 4.3 and measured the height, weight and fitness of 800, 9-11 year old primary school children across Swansea ${ }^{402}$. The study showed that $26.8 \%$ of school children were overweight or obese and $50 \%$ were unfit ${ }^{395}$. Therefore, 241 children from participating schools matched the criteria for initial screening into the study. The aim was to recruit up to fifty children from the City and County of Swansea through announcement flyers distributed at primary schools, referral by paediatricians from secondary care units and advertisements in school newsletters. 


\subsubsection{Schools}

Contact was made with 13 schools from around Swansea, including those participating in Swanlinx. Each school was asked if they would allow us to 1) Visit the school and talk to the children about the study; 2) Distribute flyers to the students to be taken home to parents and 3) Include an article in the school newsletter or website.

Following a poor response from schools, it was decided to broaden the recruitment approach. Ethical approval was obtained to allow a more direct approach to the children identified through the Swanlinx program, the use of social media, to recruit through primary and secondary care centres, and to air a radio advert. After taking part in the Swanlinx study, each school was sent a preliminary report outlining the number of children that were overweight and unfit and provided information on the PREVIEW intervention to address the problem. A letter was provided to the school to be sent home to all the parents of the children who were overweight or obese (Appendix 6). The letters and postage were provided. Of the 12 schools approached, 8 agreed to send out letters and a total of 226 letters were sent.

\subsubsection{General Practitioners}

Previous research has pointed to the importance of building relationships with health professionals and the presence of a trusted adult in the recruitment of overweight and obese children to a weight management program ${ }^{400}$. Therefore, health professionals such as GPs are important in the recruitment process.

Eleven GP practices located around Swansea were contacted and asked to participate. A poster and flyers were provided to be displayed in the GP practice and the GP was made aware of the program so as to be able to refer potential participants. There was no response to emails sent to practice managers and GPs. Of the 9 GP clinics visited in person all 9 agreed to take posters and flyers.

The Primary Care Research Incentive Scheme (PiCRIS) is a program run by Health and Care Research Wales aimed at increasing the engagement of general practices in high quality research by providing financial incentives. A meeting with the regional representative of the PiCRIS program resulted in a message being sent to all participating GP surgeries. The consensus among GPs was that 
data on height and weight for children in the target age range was not commonly available on GP databases and the number of overweight and obese children attending GP surgeries was low. Three practices agreed to display promotional materials and refer children on an opportunistic basis.

\subsubsection{Secondary Care}

A Singleton Hospital paediatrician promoted the study by signposting potential patients to the study on an opportunistic basis and displayed promotional material in waiting rooms of the paediatric ward at Singleton Hospital. My colleague and I attended the diabetes clinic run at Morriston Hospital. Patients attending the clinics were informed about our program and those who expressed an interest were handed flyers.

\subsubsection{Media}

A press release was produced in conjunction with the University press officer and sent to local media outlets. Consequently, two local papers included small articles about the study and it was mentioned on the local radio station news.

Paid advertisements were run on local Swansea radio station (The Wave), 5 plays per day for $25^{\text {th }}$ March $1^{\text {st }}$ April (advert script Appendix 7). An electronic billboard poster was displayed at Swansea bus station for a duration of two weeks. 


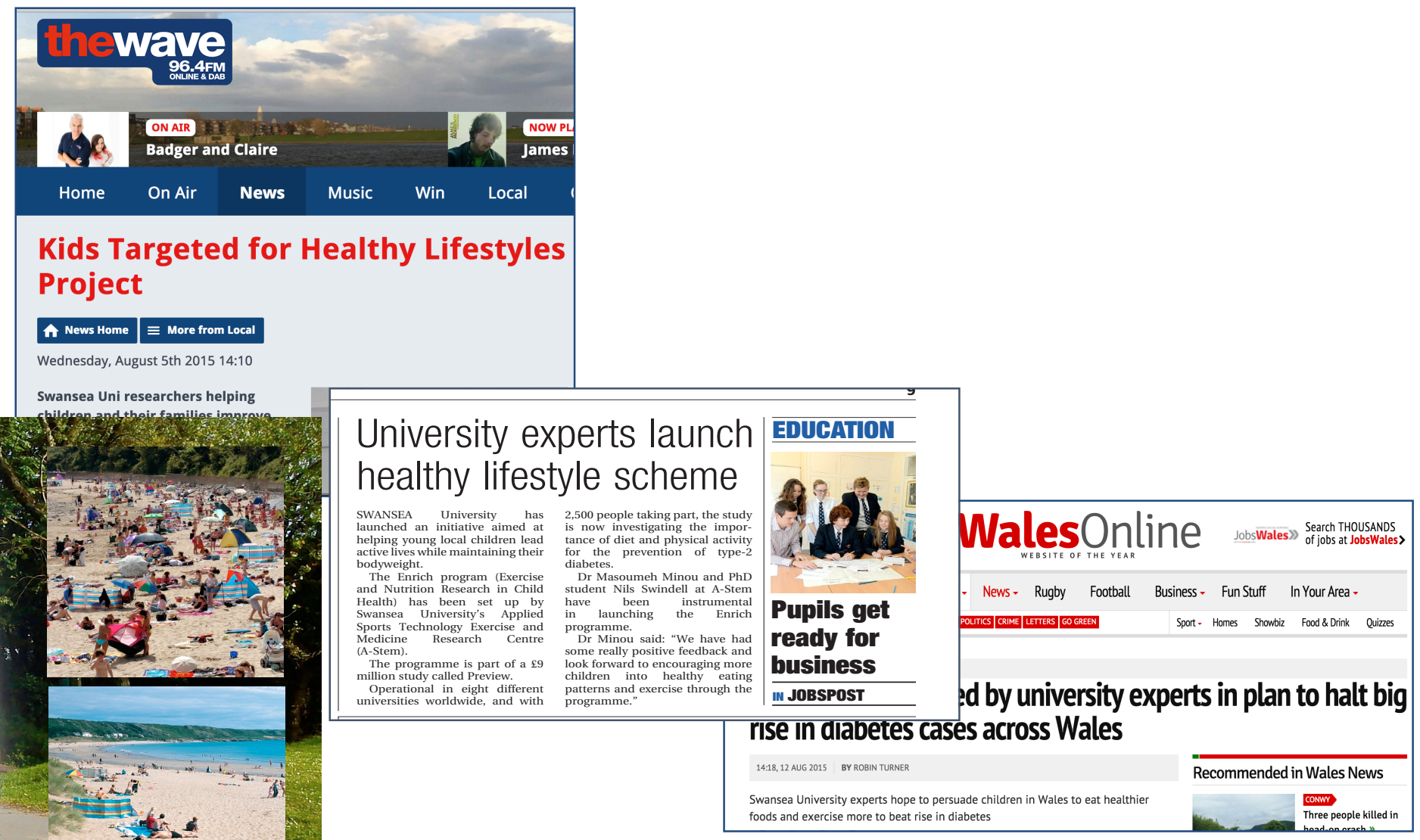

\subsubsection{General advertising}

Posters and flyers were displayed in local businesses including Asda, Tesco, Sainsbury, DVLA, Amazon and McDonalds.

\subsubsection{Recruitment events}

Research staff hosted a seminar inviting community members such as dieticians, doctors, healthy schools network team and the public health director for Swansea. The aim of the seminar was to inform community health professionals about the program and seek assistance in recruitment.

Research staff attended Swansea's annual healthy schools network meeting and presented preliminary finding of the first study participants and asked healthy school coordinators to promote the study in their schools.

\subsubsection{Results and discussion}

During the 7-month recruitment period, 20 potential recruits contacted the study, 11 attended an information meeting and finally 9 were recruited. 
Figure 8. 2 Recruitment pathways and response rate

\begin{tabular}{|c|c|c|c|c|c|c|}
\hline Schools & $\begin{array}{l}\text { Letters to } \\
\text { parent }\end{array}$ & $\begin{array}{l}\text { Poster in GP } \\
\text { surgeries }\end{array}$ & $\begin{array}{l}\text { Doctor } \\
\text { referrals }\end{array}$ & $\begin{array}{l}\text { Posters in } \\
\text { public } \\
\text { buildings }\end{array}$ & $\begin{array}{l}\text { Radio } \\
\text { advert }\end{array}$ & $\begin{array}{l}\text { Secondary } \\
\text { care }\end{array}$ \\
\hline 2 & 4 & 2 & 0 & 1 & 9 & 2 \\
\hline & & \multicolumn{3}{|c|}{ Made contact 20} & & \\
\hline & & \multicolumn{3}{|c|}{ Attended information meeting } & & \\
\hline & & \multicolumn{3}{|c|}{$\begin{array}{c}\downarrow \\
\text { Signed inforn } \\
9\end{array}$} & & \\
\hline
\end{tabular}

Considering the primary recruitment drive was aimed at schools supported by prevalence data on obesity rates and the existing relationship between the University and the schools, it came as a surprise that recruitment through this avenue was unsuccessful. Contrary to reports by Nguyen et al. ${ }^{401}$, who found school newsletters were the most successful recruitment approach, of the 13 schools we contacted only 1 agreed to include an article in the newsletter. Feedback from head teachers and school staff consistently advised us that information about the study should be directed at parents and not children and that it is not preferred by teachers to address the whole class on an issue that only concerns a few as this is not a productive use of time and also runs the risk of stigmatising overweight individuals.

General practice surgeries did not refer a single participant to the study. This was disappointing considering the GP's position in the community and the potential for recruitment. Previous studies have reported poor uptake of overweight and obese children and adolescents from GP referral ${ }^{399,401}$. In a Canadian study, GPs reported finding it difficult to approach potentially eligible subjects on the sensitive subject of weight ${ }^{399}$. Feedback from local GPs suggested that although there were many potentially eligible children in the area, very few visit GP surgeries with complications at an early age.

While the figures reported here represent the response to recruitment efforts during the 7-month recruitment period, potential participants continued to make enquiries after recruitment was closed. While this may partly reflect the fact that recruitment materials were still on display in community buildings, it is also 
possible that it takes time for awareness of a program to spread and for individuals to give it consideration before stepping forward to take part. Morrison and colleagues ${ }^{400}$ identified the importance of involving trusted adults and community professionals in the recruitment process. In the present study, one teacher (healthy school coordinator), one paediatrician and one community health care officer were particularly proactive on behalf of the study. However, it took time to build these relationships and establish a new service where previously none was available. While Nguyen et al. ${ }^{401}$ identified word of mouth as a low-cost recruitment pathway, feedback received from participants in the present study suggests that a reluctance to discuss body weight issues may hinder this approach.

[Parent about her son] "That's a difficult question for me because I don't think he talks to his friends about it [the program] at all”

"Would you recommend the program to somebody else?"

[parent] "It's a difficult one because I think the presumption would be if you did that that it was a child who had a weight problem. I would be very cautious about doing that. If somebody says, is it a good thing to do? I would have said yes but I don't think I volunteer it."

\subsubsection{Conclusion}

Recruitment was challenging, time consuming and labour intensive. The radio advertisement was the most successful recruitment approach but also the single largest financial outlay. Building relationships with community professionals and establishing an intervention as a suitable treatment pathway takes time. This should be considered when deciding on an appropriate recruitment time frame.

\subsection{Methods}

A mixed methods design was used to collect data regarding the effectiveness of the PREVIEW weight management intervention from children and their parents. The overall PREVIEW project methods are described in chapter 4.2. Quantitative data were used to describe participant characteristics and track changes in risk factors over the two-year intervention. Qualitative data were used to show participants' and their parents' perceptions of behaviour change and response to the intervention. 


\subsubsection{Participants and settings}

In June 2015, 9 children, age 10-12 years, were recruited from Swansea and the surrounding area (UK) through radio advertisements, newspaper articles, local schools and GP referral. Participants were overweight or obese according to the International Obesity Task Force criteria (IOTF) ${ }^{293}$ and insulin resistant, defined as HOMA-IR $>2{ }^{403}$. Ethical approval for all the measures was obtained from both the University R\&D (Research and Development) and the Wales Research Ethics Committee (REC). Signed informed assent was received from all children and consent from their parent or guardian.

\section{Qualitative methods}

Semi-structured interviews were conducted at 6 months, 18 months and 24 months ${ }^{404}$. Children and their parents were interviewed in separate rooms to remove the influence of parents on children's answers and vice-versa. The interview questions were based on the behaviour change techniques outlined by the PREMIT intervention that were delivered through the progressive stages of the study. (A list of questions for each interview can be found in the Appendix 8). Three interviews were conducted addressing the objectives and concepts that were relevant to the stage of the intervention. Interview 1: addressed identification of target behaviours, adoption of new healthy behaviours, relatedness, vulnerability, social support, goal setting, self-monitoring, visualisation techniques and competency. Interview 2: addressed maintenance, relapse, attribution, social support, self-efficacy, competency, action planning, self-monitoring. Interview 3: addressed maintenance, relapse, self-efficacy, competency, action planning, self-monitoring, goal adjustment.

Interviews were transcribed verbatim for further analysis. Qualitative data were subsequently analysed to explore the views and determine themes from participants. The aim was to determine to what degree participants had been compliant with the intervention objectives and how participants had interpreted and responded to the behaviour change techniques used throughout the intervention. First transcripts were analysed through a deductive process that reflected the behaviour change objectives and techniques used. The themes of the analysis were pre-determined by the objectives and content of the 
intervention. For example, themes included, action planning, goal setting, vulnerability, outcome expectancy and self-efficacy. The verbatim transcripts were read through and each sentence was coded according to its appropriate theme. Finally, the copy and paste technique was used to combine all quotations for each theme. Transcripts were then examined inductively to allow the emergence of themes that were worthy of attention ${ }^{405}$. To ensure methodological rigour and enable co-operative triangulation, verbatim quotations were presented by the first author (NS) and to the second author $(\mathrm{MM})$. Analysis was critically questioned and data cross-examined, finally the process was run-in reverse working back from the interpretation of verbatim quotations to the original transcriptions. This process was repeated until a consensus was met. Finally, the first and second author interacted with the children and their families throughout the delivery of the intervention, building relationships that subsequently increased the credibility of the reporting and interpretation of the results.

\subsection{Results}

A total of 9 participants were recruited and completed baseline measurements (Table 4.1), 2 participants dropped out before starting the intervention phase, and another dropped out before the second measurements were taken. A complete case analysis of the 4 participants who completed the two-year intervention is presented below.

Table 4. 1 Baseline characteristics of 9 participants recruited to the lifestyle intervention

\begin{tabular}{lllllllll}
\hline Participant & Sex & $\begin{array}{l}\text { Age } \\
(\text { years })\end{array}$ & $\begin{array}{l}\text { BMI } \\
\left(\mathrm{kg} \cdot \mathrm{m}^{2}\right)\end{array}$ & $\begin{array}{l}\text { BMI- } \\
\text { Z }\end{array}$ & $\begin{array}{l}\text { Waist-hip } \\
\text { ratio }(\mathrm{cm})\end{array}$ & $\begin{array}{l}\text { Fat } \\
(\%)\end{array}$ & $\begin{array}{l}\text { HOMA- } \\
\text { IR }\end{array}$ & $\begin{array}{l}\text { HbA1c } \\
(\%)\end{array}$ \\
\hline A & M & 13.2 & 28.58 & 1.51 & 0.83 & 47.3 & 2.96 & 5.5 \\
B & M & 10.8 & 33.83 & 3.3 & 1.02 & 59.3 & 5.79 & 5.7 \\
C & M & 13.0 & 22.3 & 1.42 & 0.91 & - & 3.8 & 5.3 \\
D & F & 12.6 & 28.27 & 2.35 & 0.94 & 45.2 & 4.19 & 4.5 \\
E & M & 10.1 & 24.9 & 2.54 & 1.00 & 45.6 & 2.74 & 5.6 \\
F & F & 11.4 & 24.46 & 1.97 & 0.86 & 37.4 & 1.73 & 5.6 \\
G & M & 12.1 & 31.51 & 2.95 & 0.99 & - & 5.65 & 5.4 \\
H & F & 11.5 & 29.31 & 2.65 & 0.91 & 39.7 & 4.16 & 5.7 \\
I & M & 13.3 & 33.97 & 3.07 & 0.91 & 34.8 & 6.16 & 5.3 \\
\hline
\end{tabular}


Figure 8. 3 Changes in BMI z-score over the two-year intervention BMI z-score for participants A-F at each time point

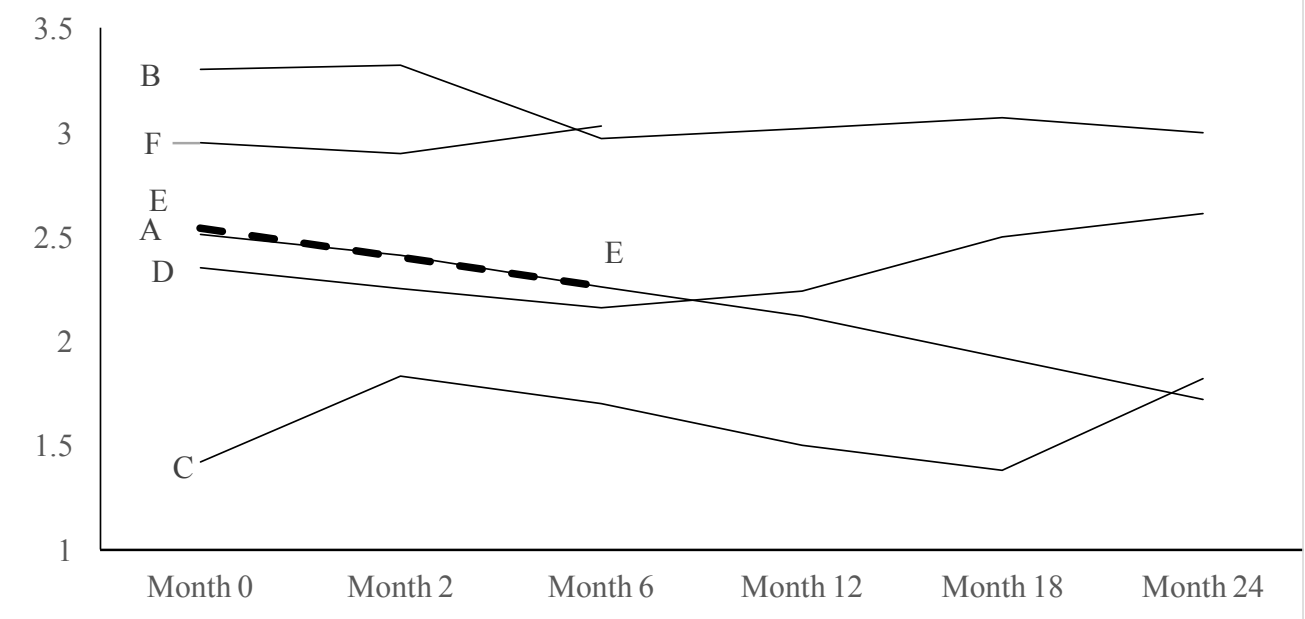

Table 4. 2 Participant A

\section{Baseline}

Participant A was male and 13-years old at the start of the study. He resided with his mother and sister in the Swansea area. When joining the study, he had a BMI z-score of 2.15 and a HOMA-IR of 2.96 . He spent on average 57 minutes per day in MVPA, mean counts $\cdot$ minute $^{-1} 398.9$ and 655.1 minutes per day in sedentary behaviours. Participant A attended most of the meetings with his mother who offered a positive presence providing discussion and input for her son and the other participants. Participant A appeared shy in the group meeting but as one of the older participants he showed a good understanding of the content and aims of the study.

\section{6 months}


At 6 months, participant A had reduced his BMI z-score to 2.26, a 10\% reduction. The accelerometer output indicated that he did not increase his physical activity, spending $6 \%$ less time in MVPA and 14\% less time in overall body movement $\left(\right.$ counts $\cdot$ minute $^{-1}$ ). Participant A spent an average of 46 minutes or $7 \%$ more time in sedentary behaviours. Food diaries showed no change in macronutrient composition, and very little change in other parameters. There was a small reduction (7\%) in average daily GI.

\section{Months}

At 12 months the accelerometer output showed a marked increase in MVPA $(+23 \%)$ and total body movement $(+11 \%)$ from baseline. Sedentary time had decreased from the 6-month assessment but was still 5\% above baseline. The food diary showed a marked reduction in free sugars $(-71 \%)$ but little change in other parameters.

Participant A's BMI-Z score had further reduced to 2.12, a reduction of $16 \%$ from baseline.

\section{4 months}

At two years, participant $\mathrm{A}$ had further reduced his BMI z-score to 1.72 , a reduction of $31 \%$ from baseline. His HOMA-IR had reduced to 1.47 and his HbAlc had reduced to $5.3 \%$, a reduction of $50 \%$ and $4 \%$, respectively. The accelerometer data suggest that on average 69.9 minutes per day was spent in MVPA, an increase of $23 \%$ from baseline. Total body movement was at its highest level recorded over the 2 years, a 16\% improvement from baseline. Food diaries indicated an increase in protein and fat intake and a subsequent reduction in carbohydrate intake. Free sugar intake remained below baseline (-42\%) and the GI had reduced by $13 \%$. 
Table 4. 3 Participant $B$

\section{Baseline}

Participant B is male and 10-years old at the study onset. He resides predominantly with his grandmother with whom he attended all the meetings. His mother also lives locally and attended two meetings at the beginning of the study. $\mathrm{He}$ is quiet during meetings and requires some encouragement to give answers or take part in group exercises.

When joining the study he had a BMI-z-score of 3.3, his HOMA-IR was 5.8 and HbA1C 5.7; participant $\mathrm{B}$ is pre-diabetic by American Diabetes Association criteria $^{406}$. He spent on average 44.4 minutes per day in MVPA and 658 minutes in sedentary behaviours.

\section{6 months}

At 6 months, participant B had a 10\% reduction in his BMI z-score. This was accompanied by a 79\% reduction in HOMA-IR (1.2) and $4 \%$ reduction in HBA1c (5.5).

The accelerometer data showed he spent 23\% more time in MVPA and recorded $11 \%$ more total body movement while sedentary time remained constant. The food diary showed a greater contribution of total energy from protein (from 15.2\% to $17.6 \%$ ) but $172 \%$ increase in free sugars and $10 \%$ increase in GI.

\section{Months}

At 12 months, participant B's BMI z-score slightly increased but was still an 8\% reduction from baseline. HOMA-IR had increased but was still $40 \%$ reduction from baseline. HbA1c continued to fall and was a $9 \%$ reduction from baseline.

Total body movement had increased and was 39\% higher than baseline. MVPA had also increased to 69.4 minutes per day, a 55\% improvement from baseline. Sedentary time had made a small reduction $(-2 \%)$.

Participant B was unable to return a valid food diary. 
At 2-years we had not seen the participants for 6 months, he has visited the research centre for his final measurements but have not received any guidance or feedback during this time. Participant B had a BMI z score of 3, a 9.1\% reduction from baseline. Following an increase at 12 and 18 months, HOMA-IR had fallen to 2.86 , down $51 \%$ from baseline. HbAlc had reduced to 5.2 , indicating that participant B was no longer classified as pre-diabetic ${ }^{406}$.

Accelerometer data suggest more time was spent in MVPA $(65 \%$ increase from baseline) and total body movement (37\% increase from baseline).

The food diary showed a higher intake of free sugars $(+14 \%$ from baseline), GI $(+36 \%$ from baseline) and a higher contribution of energy from protein up to $20.6 \%$ in line with the study objectives.

Table 4. 4 Participant $C$

\section{Baseline}

Participant $\mathrm{C}$ is male and 12-years old at the study onset. He resides with his mother, father and older brother in the Swansea area. At baseline, he had a BMI-zscore of 1.42 and was classed as overweight by IOTF criteria ${ }^{293}$.

He had a HOMA-IR of 3.8 and HbA1c 5.3.

He spent an average of 65.8 minutes per day in MVPA and spent 665 minutes per day in sedentary behaviours.

\section{6 months}

At 6 months, his BMI z-score had risen to 1.7, an increase of 20\%. HOMA-IR dropped by $19 \%$, while HBA1c reduced by $5 \%$.

The accelerometer data showed that participant $\mathrm{C}$ was less active at 6 months, spending on average 20 minutes less in MVPA (40\% reduction) and recording a $38 \%$ reduction in total body movement. Time spent in sedentary behaviours increased by 29 minutes (4\%).

The food diary showed an increase in the relative contribution to total energy from protein and fat and a subsequent reduction in carbohydrate; there was an $11 \%$ reduction of free sugars but also a $32 \%$ reduction in fibre. Average daily GI had reduced by $22 \%$. 


\section{Months}

At 12 months, a BMI z-score of 1.5 was a reduction from 1.7 at 6 months but remained $6 \%$ above baseline. HOMA-IR had reduced to 2.7 while HbA1c remained at 5\%. Total physical activity (counts·minute ${ }^{-1}$ ) and MVPA had increased from 6 months but remained below baseline levels. Counts per minute were $25 \%$ below baseline and MVPA was $23 \%$ below baseline. Sedentary time had reduced but remained 11 minutes (2\%) above baseline. Participant $\mathrm{C}$ was unable to return a valid food diary at 12 months.

Shortly before the 12-month meeting, participant $\mathrm{C}$ was having doubts about continuing with the program and his mother called a meeting to talk to the research team. His mother was concerned that nothing had changed in her son's attitude, he was not following the advice given by the study nor had he changed his behaviour. Despite this, participant $C$ wanted to continue with the study.

\section{4 months}

At 2-years we had not seen the participant for 6 months, he visited the research centre for the final measurements but had not received any guidance or feedback during this time. Participant C's BMI z-score had increased to 1.83, its highest point during the study and a $28 \%$ increase from baseline. HOMA-IR had also increased to 4.31 , a $13 \%$ increase from baseline. HbAlc increased to $5.6 \%$. Total physical activity and MVPA had increased with participant $\mathrm{C}$ achieving over 60 minutes of MVPA per day for the first time since baseline. Sedentary time declined from 12 months but remained similar to baseline.

The food diary showed an increase in free sugar intake $(143 \%$ higher than baseline). Fibre intake had reduced from 12 months and remained 14\% below baseline. GI was the highest recorded over the 2 years. After 2 years, participant $C$ still ate sugary chocolate cereal for breakfast, biscuits and white bread for lunch at school, cola and deserts, suggesting that his behaviour had not changed despite what was discussed in the meetings.

Table 4. 5 Participant D

\section{Baseline}


Participant D is female, 12 years old at baseline and lives with her mother in the Swansea area. When joining the study she had a BMI z-score of 2.35, a HOMA-IR of 4.2 and HbA1c of 5.4 .

She spent 71 minutes in MVPA and 683 minutes in sedentary behaviours every day.

$61 \%$ of her energy intake came from carbohydrate and she consumed $82 \mathrm{~g}$ of free sugars. The main contributors to free sugars were cola, fruit juices and chocolate bars.

6 months

At 6 months, participant D had reduced her BMI z-score to 2.25, a reduction of $8 \%$.

HOMA-IR had increased to 4.82 but Hbalc had fallen by $5 \%$ to $5.2 \%$.

The accelerometer output indicated that she had become less active, spending $23 \%$ less time in MVPA, 13\% less overall body movement (counts $\cdot$ minute $^{-1}$ ) but she had also spent on average 18 minutes per day less in sedentary behaviours. Participant D's food diary showed a greater contribution to total energy from protein and fat and a subsequent reduction in carbohydrate (see table 4.8). There was a $79 \%$ reduction in free sugar intake and an $11 \%$ reduction in average daily GI.

\section{Months}

At 12 months, participant D's BMI z-score had risen to 2.24 but remained 5\% below baseline.

HOMA-IR had fallen and was $11 \%$ below baseline. Hba1c continued to fall and was at $5.1,6 \%$ below baseline. The accelerometer data suggested she was spending more time in MVPA, above 60 minutes per day and 1\% above baseline levels. Total physical activity was still below baseline. Sedentary time was 63 minutes per day higher than baseline. Participant D was unable to complete and return a valid food diary.

\section{4 months}

At 2 years, participant D's BMI z-score had risen to 2.61, $11 \%$ above its baseline value. HOMA-IR has increased again to 4.58 , 9\% above baseline. HbA1c had also increased and was identical to baseline at $5.4 \%$. The accelerometer data suggested that she spent $20 \%$ less time in total activity and $19 \%$ less in MVPA. 
Sedentary time had declined and was $3 \%$ below baseline. Participant $\mathrm{D}$ was unable to return a valid food diary.

Table 4. 6 Changes in physical activity and sedentary time over 2 years

\begin{tabular}{|c|c|c|c|c|c|}
\hline \multirow[t]{2}{*}{ Participant } & \multicolumn{5}{|c|}{ MVPA (minutes $\cdot$ day $^{-1}$ ) } \\
\hline & 0 months & 6 months & 12 months & 18 months & 24 months \\
\hline A & 57.0 & 53.7 & 70.3 & 51.6 & 69.9 \\
\hline B & 44.7 & 54.9 & 69.4 & 51.9 & 73.8 \\
\hline $\mathrm{C}$ & 65.8 & 39.4 & 50.0 & 50.7 & 83.1 \\
\hline \multirow[t]{2}{*}{$\mathrm{D}$} & 71.6 & 55.3 & 72.4 & 59.7 & 57.9 \\
\hline & \multicolumn{5}{|c|}{ Counts per minute (counts $\cdot$ minute $^{-1}$ ) } \\
\hline A & 398.9 & 343.4 & 443.9 & 373.5 & 462.6 \\
\hline B & 367.3 & 408.2 & 496.4 & 390.5 & 504.8 \\
\hline $\mathrm{C}$ & 440.4 & 271.4 & 349.0 & 328.1 & 492.2 \\
\hline \multirow[t]{2}{*}{$\mathrm{D}$} & 465.1 & 398.0 & 447.4 & 407.6 & 372.1 \\
\hline & \multicolumn{5}{|c|}{ Sedentary time (minutes $\cdot$ day $^{-1}$ ) } \\
\hline A & 655.1 & 701.6 & 726.1 & 684.7 & 689.8 \\
\hline B & 658.5 & 655.7 & 643.9 & 637.9 & 683.3 \\
\hline $\mathrm{C}$ & 655.5 & 684.0 & 685.0 & 667.8 & 653.1 \\
\hline $\mathrm{D}$ & 683.1 & 664.9 & 744.8 & 637.9 & 661.1 \\
\hline
\end{tabular}


Table 4. 7 Changes in HOMA-IR and Hbalc over two years

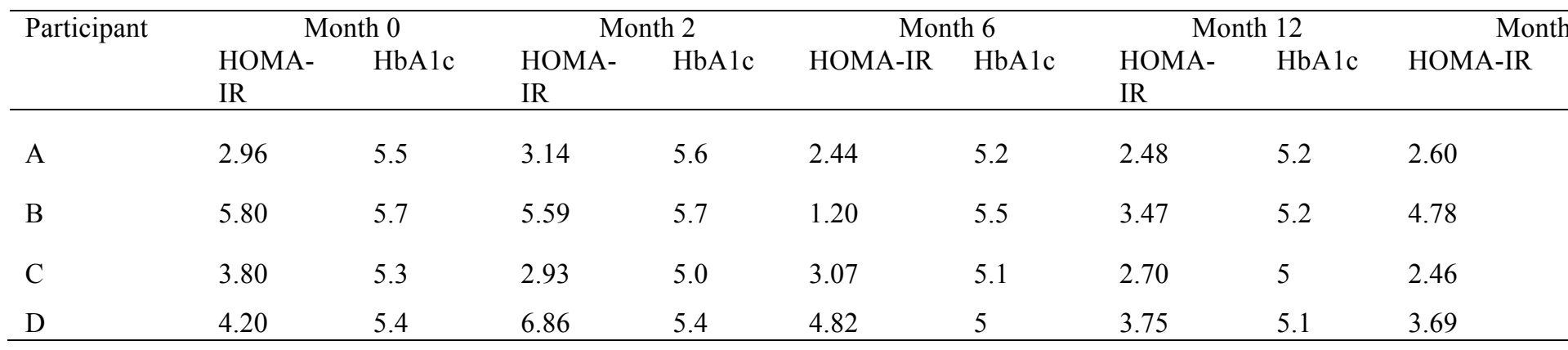

Table 4. 8 Changes in dietary intake over 2 years

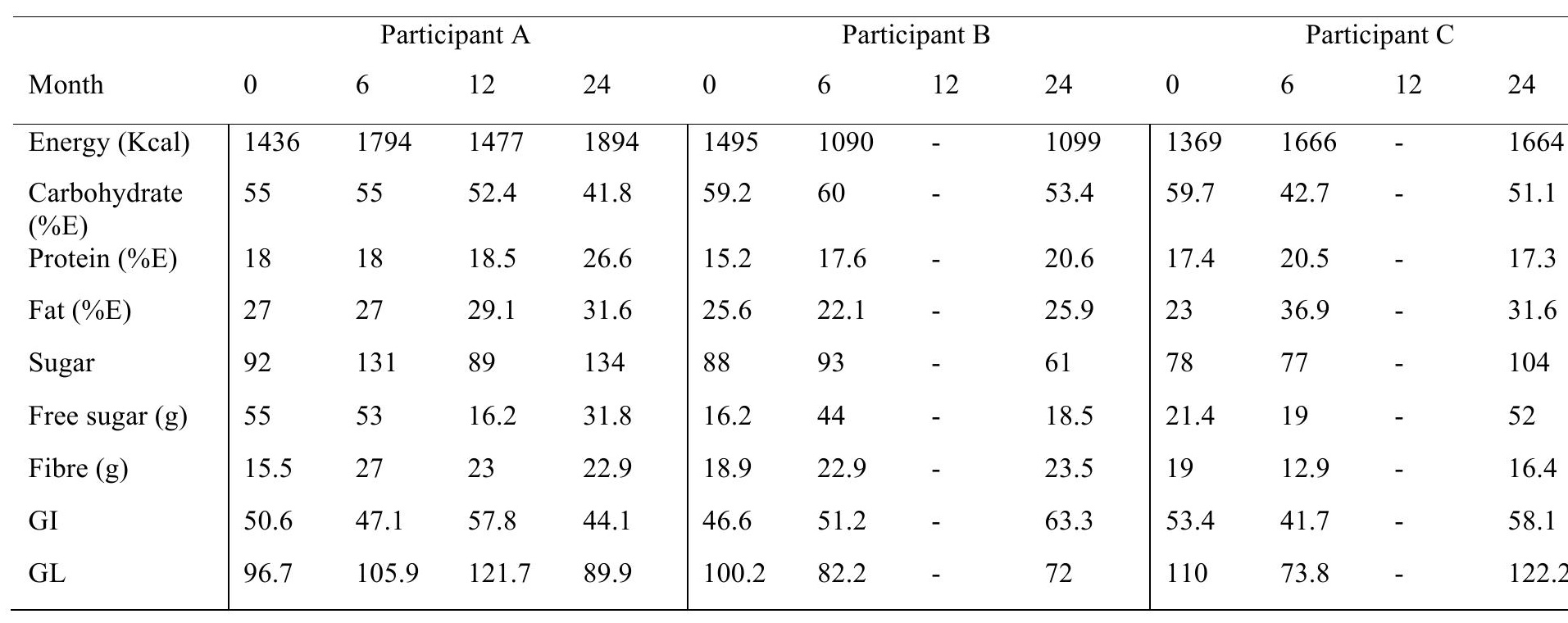




\subsection{Discussion}

The child and parent's perspective of the behaviour change process provides context to the successes and failures during the intervention, offering an insight into their interpretation of a theory driven intervention and their compliance to the objectives.

\section{Objective measures vs participant perceptions}

The lifestyle intervention produced a sustained reduction in BMI z-score in two of the four children. Participants A and B achieved a $>9 \%$ reduction in BMI zscore which was sustained over a two-year period. In both cases the reduction in BMI z-score was accompanied by a reduction in HOMA-IR and HbA1c. The accelerometer and food diaries captured changes in dietary and physical activity behaviours of participant $\mathrm{A}$ and $\mathrm{B}$, in line with their perceived changes which may explain the physiological outcomes. However, this was not the case at each time point. All the children and their parents/guardians professed to having increased their knowledge and awareness of healthy behaviours and to have made positive changes.

Participant A: "Food, changed bread, changed my snacks, we have stopped buying biscuits and flapjacks, I have Ryvita or chilli pepper stuff with cottage cheese which is quite nice."

Participant B: "I'm doing more exercise than what I used to do, and I'm eating healthier than what I used to."

Participant D: "Me and my mum have made a little routine for activity on the diary planner thing and we've gotten into a routine of we know what we got to be doing exercise wise." (More quotations in appendix 9)

These changes were not always detectable with our objective measures. At 6 months only participant B had increased his total physical activity and MVPA, in agreement with his and his Grandma's perception. The three remaining participants (A, C and D) spent less time in MVPA and recorded fewer counts $\cdot$ minute $^{-1}$. Participants A and D gave clear examples of new active behaviours they had undertaken and their parent agreed they had become more active. Previous studies have reported that when adding physical activity to a child's day, they can compensate by being less active in the rest of the day ${ }^{407}$. It 
may also be important to add that baseline measurements were taken in June when daylight hours were longer and weather may have been more supportive of outdoor activity than in December. Children's physical activity has been shown to vary seasonally ${ }^{408}$. Therefore, it is possible that although participant A and D had added structured exercise to their schedule, due to compensation or a seasonal decline, their net physical activity levels had fallen. Participant $\mathrm{C}$ did not make a visible change to his dietary or physical activity behaviours which matched his mum's assessment.

Researcher: "How has it [the program] influenced your son's attitude, skills, knowledge or, behaviour?

Parent C: "I don't know that it has."

Although participant $\mathrm{C}$ provides examples of activities he has undertaken (walking to school, cycling) these were not new behaviours.

Parent C: "Walking to and from school he has always done anyway"

At 12 months, all participants recorded an increase in MVPA and total physical activity. However, it was also a return to summer; participants A, B and D recorded higher MVPA and counts $\cdot$ minute $^{-1}$ than baseline. Tracking changes in dietary intake was problematic due to poor compliance with food diaries.

Participant D made measurable changes to dietary and physical activity behaviours, experiencing a reduction in BMI z-score at 6 and 12 months. Unfortunately, this was not sustained at 2 years when BMI z-score and HOMAIR were higher than baseline. Despite acknowledging the relapse, participant D and her mum believed they had made positive change and were able to recover from the relapse.

Parent D: "She has seen previously how good she has felt, how good the changes have been and she wants that again. So it's that drive now. The last couple months haven't been good but she wants to get to that point again. She is definitely enthusiastic for it which is always half the battle." (More quotations in appendix 9)

\section{Participant perception of behaviour change objectives}

Vulnerability, outcome expectancy

The main objectives of the preparation phase were to foster the idea of vulnerability associated with poor diet and inactivity and develop a positive 
outcome expectancy of adopting behaviour change. Participants A and B and their respective guardians clearly showed their perceived vulnerability based on their weight status and lifestyle behaviours.

Researcher: "How important do you think your healthy behaviour is in preventing disease?"

Participant A: "Very, very important because um... basically like stop me from getting it. If I was going to go on the way I was I was probably getting it. [diabetes]"

Participant B: "In the beginning I nearly had it [Diabetes] but it was good timing I came into this program because I almost had it and since then I don't eat as much sweets with that much sugar in them."

In contrast, participant $\mathrm{C}$ stated that he was no longer at risk as he believed he had lost weight.

Researcher: "Do you see yourself as being at risk?"

Participant C: “Um.... I did at one point but now I don't think I am at risks anymore" "Quite good, I've lost a lot of weight thanks to this [the study]."

This perception was not supported by changes to his BMI z-score, nor were there any signs of behaviour change in the accelerometer or food diary results. It was unclear whether participant $\mathrm{D}$ perceived herself to be vulnerable to diabetes as she referred to her weight as her desired outcome of behaviour change and not the prevention of illness. In contrast to their children, parents of participant's C and $\mathrm{D}$, were clear that their children were at risk due to their weight status and activity behaviours.

Parent C: "I don't think he is at risk because of my husband but I do think he is at risk because he is overweight and not active."

Researcher: "Do you think she is at risk?"

Parent D: "Yeah - the blood test results frightened me"

The trans-theoretical model ${ }^{409}$ distinguishes four stages, starting with a stage of unconsciousness, where vulnerability is unknown. During subsequent stages the risk of the behaviour becomes clear and new behaviours are adopted and eventually habituated during the proceeding stages. Meta analyses have shown fear appeal to be effective in influencing attitude, intentions, and behaviours 410 and perceived severity and susceptibility ${ }^{411}$, although primarily in adults. In the 
present study, child sensitive fear appeal techniques were used to foster a sense a vulnerability by explaining to participants the likely consequences of obesity, inactivity and poor diet. It may be reasonable to assume that participants, or at least their parents, are aware of their vulnerability to have signed up to a diabetes prevention program. In the cases presented here, all parents indicated vulnerability. However, even after the preparation phase, participant $\mathrm{C}$ did not indicate that he felt vulnerable which may explain why he did not go on to produce any notable behaviour change.

Goal setting and action planning

Goal setting was used throughout the study; children defined goals according to the SMART principles and were encouraged to set behavioural goals as opposed to outcome goals. These goals were discussed frequently during group meetings when children received feedback, adjusted and progressed their goals. Action planning exercises were undertaken by the children to define situational triggers and outline appropriate behavioural responses. The children signed a behaviour change contract stating their dietary and physical activity goals and outlining the steps needed to achieve them, to anticipate potential barriers and plan solutions. At 6 months, participants A, B and D perceived goal setting to have been helpful and provided examples of action planning to overcome barriers.

Researcher: "How does setting goals help you?"

Participant B: "Because it helps me think what I'm gonna do after school instead of sitting down or playing on my Xbox."

Participant D: "It does help me because it makes me feel better after we've done all the things in the planner and I can literally feel the difference after I've done all the things that I've planned makes me happy"

Participant $\mathrm{C}$ did not provide examples of goal setting but believes he is too busy. His mum believed that he did not set goals or plan physical activity.

Parent C: "He should be able to, but there is always something else to do. So I think that is proving difficult."

Participant D clearly used goal setting with a weekly activity planner written on a blackboard. Her weekly activities were part of a training schedule which culminated in a 5-kilometre run. 
Participant D: "Me and my mum have made a little routine for activity on the diary planner thing and we've gotten into a routine of we know what we got to be doing exercise wise."

Figure 8. 4 Participant D's exercise plan, demonstrating the use of planning and prompts

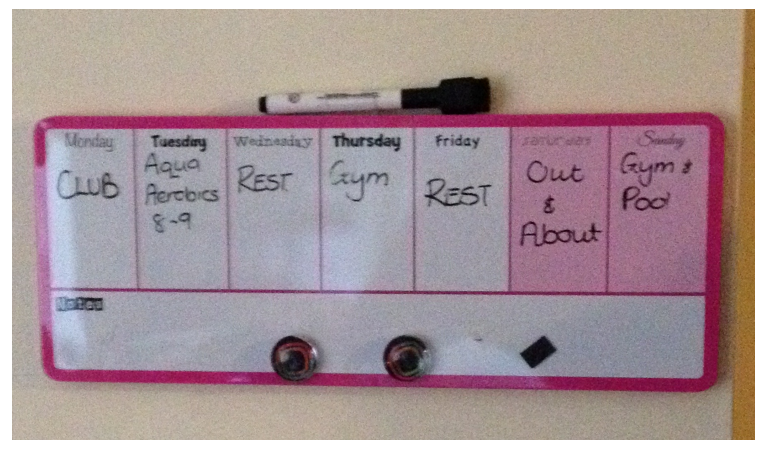

In contrast, participants A and B struggled to define written goals but made verbal goals with their parents. At 12 months, given the fading frequency of group visits the onus was on participants to continue with goal setting and monitoring their own progress.

Participants A, B and D still used goal setting but did not write them down or follow the guidelines provided during group visits. Specifically, participant A did not remember his written goals or behaviour change contract but set himself exercise goals like going to the gym every morning while on holiday or doing 100 press-ups per day.

Participant A: "It was for holidays to go to the gym once everyday"

Participant B does not remember his written goals but plans verbal goals with his Grandma and has a reward system place if he achieves his goals.

Parent B: "Communicating the goals and objectives with parents- talk about them, then go on a walk."

Participant D no longer writes a planner but signs up to running events and builds up her training before each event.

Participant D: "I set a goal on the rainbow run that I did... Working up to it was a goal." 
Although participants were encouraged to set behavioural goals, all three participants also refer to outcome goals related to body weight, body shape or clothes size.

Parent B: "He's got a goal in his mind that he doesn't want to go back to his previous weight and that keeps him motivated."

Parent D: "We monitor her weight, we monitor her height, sometimes she gets down but she doesn't realise she is growing upwards so she is getting heavier."

The objective of the study was not for children to lose body weight but to maintain a constant weight over the first 8 weeks and thereby reduce their BMI. It was repeatedly emphasised that as they were still growing they would become heavier irrespective of their body fat. Therefore, setting weight loss goals may not be realistic or achievable, participants were likely to fall short of their goals causing dissatisfaction which may in turn cause them to disengage from the goal 412

At 24 months, goal setting techniques were no longer being used, however participant A, B and D explained that they were now in a routine. Participant A had got sporting aspirations and a busy training schedule, participant B had got a verbal schedule with his Grandma and his friends, while participant D had weekly activities with her mum.

Participant A: "Like say training three times a week and going for two runs a weekend. Eating more vegetables and proteins and less fatty foods like chocolate and sweets."

Parent A: "Whether he actually sits down and plans things out or not, I'd be quite doubtful of to be honest with you but I just think it works because it's become a way of life. I don't think he does anything formal in that regard but on the other hand he's working towards that goal."

Parent B: “.... his goal is not to put on and to lose some [weight] which he has been doing. And then after this holiday, there will be more goals." Parent D: "I think she just has a mind-set. We don't really have goals anymore. It's just she knows what she is going to do. She knows when she is not trying as hard as she could. She knows when she is trying hard. She can see the differences in herself and feel the differences. "It's just 
becoming part of our weekly routine now. We don't really set goals as such.”

\section{Self-monitoring}

During the action phase, self-monitoring of behaviour was introduced. Participants were encouraged to complete physical activity and dietary logs. The use of apps, step counters, and heart rate monitoring were later introduced. A system of "plan-perform-evaluate" was initiated whereby children would plan their dietary and physical activities via their smart goals and action planners, they would perform the planned activities over the coming weeks, evaluate their performance by monitoring their behaviour, and finally, reflect on their progress and adjust their goals accordingly. During group meetings, encouraging participants to complete their dietary and physical activity logs was challenging, on occasions that they were completed it was largely by their parents. At 12 months, except for participant B who used a stopwatch to time his exercise and set himself goals to improve his lap time, participants were not using selfmonitoring techniques to monitor their behaviour.

Participant A: "I do weigh myself sometimes and I do look at pictures from before and compare them to now."

Researcher: "What about your diet like how many vegetables you have eaten?"

Participant A “Um... no.”

Researcher: "How do you know you have made progress"

Participant A "Um... the way my body feels"

Researcher: "During the study we talked about self-monitoring, do you currently use any methods of monitoring your own behaviours, goals or progress?"

Participant C: "Not all the time because sometimes I will remind myself of why I'm doing this."

Participant D: "I have started to measure my waist every few days and write it down in a book I have to keep track."

Researcher: "What about your behaviour?

"No. Not really." 
However, they did monitor various outcomes; visual appearance (participant A), weight and clothes sizes (participant B) and waist circumference (participant D). Monitoring behaviour increases awareness which is critical for behaviour change, it is essential for self-evaluation helping to reveal sources of excess energy intake or opportunities to be active. It is also essential for selfreinforcement, and has been shown to be a critical factor in the success of weight management interventions ${ }^{413}$. In the cases presented here, there was a reluctance to adopt self-monitoring techniques as they were perceived to be time consuming. To encourage compliance, child friendly methods of self-monitoring were suggested, such as mobile phone apps, step counters, fruit and vegetable wall charts. During group meetings participants who were not monitoring their behaviour were asked to do so retrospectively but when the frequency of meetings was reduced, compliance with self-monitoring was low. Research by Germann et al ${ }^{414}$ demonstrated that children in a weight loss intervention that frequently used self-monitoring techniques achieved greater weight loss than those who did not. In the present study, failing to adequately monitor behaviour may have been a barrier to effective evaluation of performance, provision of feedback and reinforcement, ultimately hindering the effectiveness of the intervention.

\section{Competency - Self-efficacy}

From the perspective of the Self-determination theory ${ }^{209}$, competency (i.e. the need to feel a sense of mastery and the ability to perform the desired behaviour) is essential to initiate and maintain behaviour change. During the study, competency was developed by teaching and developing skills such as cooking, making food choices, reading food labels and performing exercises, stretches and warmups. Self-efficacy, defined by the social cognitive theory as an individual's confidence in being able to carry out a behaviour, has also been shown to be a critical factor in successful behaviour change ${ }^{197,298}$. Self-efficacy was promoted by focusing on past success and providing feedback on successful completing of goals and target behaviours. At 6 months, all participants demonstrated that they were competent at performing the target behaviours by providing examples of their new dietary choices and activities. 
Participant A: "Yeah I think so, I'll have porridge for breakfast, a sandwich or wrap for lunch and stir fry for dinner."

Participants A, B and C also appeared confident in their own ability to perform these behaviours. Participant A displayed his competency in completing physical activities including an exercise routine using equipment he keeps in his room. He showed us his equipment, explaining his routine, displaying confidence in his own ability to perform exercise.

Figure 8. 5 Participant A's exercise equipment

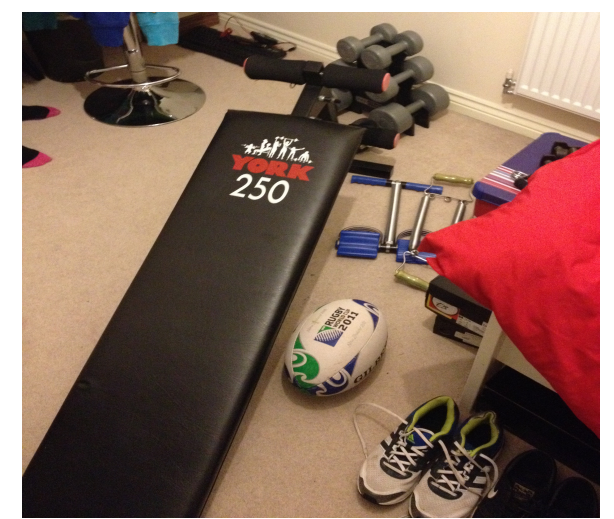

Participant B: "I am very confident to pick the right food and exercise." "Yes- like in school they have fish and chips, but I choose something like pasta and healthy options."

(More quotations in Appendix 9)

Participant $\mathrm{C}$, despite not appearing to have made any behavioural changes, perceived himself to be both competent and confident in his ability.

Researcher: Are you able to plan a healthy diet?"

Participant C: "Yes - for breakfast I'll have Weetabix because there is not a lot of sugar in it and wholegrain for lunch probably hummus, for tea I'll have salmon again because it was quite nice"

Researcher: "Are you able to plan activities for 6omin + every day?"

Participant C: "Yes quite easily, this weekend I have been doing a lot of cycling and exercise"

His mum agreed that he was capable and confident in his own ability suggesting that it was not the absence of competency or self-efficacy that prevented successful behaviour change.

Parent C: "He is very competent but I'm not sure that he appreciates why he needs to do it. I know that he does need to do it. I think in a 
theoretical way he does but I'm not sure that he applies that to his everyday life." (More quotations in Appendix 9)

Conversely, despite increasing her physical activity and making dietary changes, participant $\mathrm{D}$ ascertained that she required help making behavioural changes and was not confident in her own ability, relying heavily on her mum to motivate her and take part in physical activity with her as well as making dietary choices.

Participant D: “I don't find it difficult when I got my mum to motivate me and stuff, she is basically like a saviour because if I did it on my own I wouldn't have a clue what to do"

Parent D: "No she has other difficulties so she can't do it, she'll help, when I'm cooking something or planning something but she can't foreplan it herself."

\section{Coping self-efficacy maintenance and relapse}

After 6 months, participants had reached the maintenance phase, having started new behaviours, coping and self-efficacy ${ }^{415}$ (believing in the ability to overcome barriers or difficult situations) became the focus of the intervention. All participants provided examples of their ability to overcome relapse or temptations. Participant A was confident in his ability to overcome a relapse and called on social support to help him get back on track. Participant B had put steps in place to avoid the temptation to be inactive and recovered from relapse by discussing a new plan with his Grandma. Participant $\mathrm{C}$ managed disruption in his usual activities by replacing them with new activities.

Researcher: "Do you feel able to overcome it if you were to have another relapse?"

Participant A: "Yeah definitely"

Researcher: "What would you do?"

Participant A: "I would probably get help from my mum because like she worked for me and it helps me a lot and I would try my best to get back in the gym again and eat healthy."

Participant D stated that she was confident in overcoming a relapse by remembering the progress she had made and the medals she received for taking part in running events. However, she also disclosed that she was reliant on her mother to pick her up from a relapse. 
Figure 8. 6 Participants D's medals for participation in fun runs

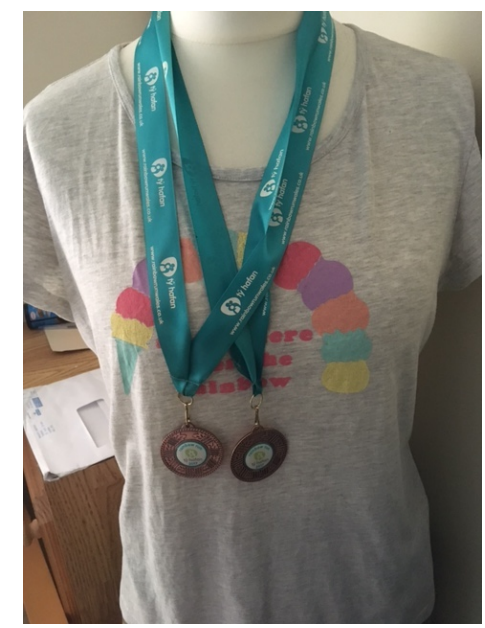

Participant D: "If I were to have another relapse I definitely think I could overcome it from the previous relapse that I have had and I know how to reverse relapse."

Participant D: "Because of the medals and stuff that I have won from the races and I would think about the stuff I have achieved over the past year and think about like how have I achieved them and if I continue doing the stuff I am and it will help me to be motivated for the future"

At 24 months, participants $\mathrm{A}, \mathrm{B}$ and $\mathrm{C}$ remained confident in their ability to overcome difficult situations. Parents of participants A and B resoundingly agreed and suggested the changed behaviours had become a way of life.

Researcher: "How confident do you think he is in his own ability to continue with these healthy behaviours?"

Parent A: "Very confident I don't think even he would think about it twice now. It's just a way of life for him."

Parent B: "Yes because he's the one that is controlling it really. He controls it more than what I do. Very much up there."

At 24 months participant D and her mum acknowledged that they were experiencing a relapse. The underlying cause was a knee injury sustained by her mother which prevented them exercising together. She also disclosed that she was prone to yielding to food temptations. Although action plans had been put in 
place, showing the ability to cope with difficult situations, they were largely initiated by her mother, further highlighting the reliance on her mother and a possible lack of coping self-efficacy.

Participant D: "I'm still struggling on the path where my friends are eating junk and stuff in school. I have a bit of a relapse in eating a bit of junk. But recently over the past few weeks haven't really ate junk. I have gotten better. My mum started giving me packed lunches with salads wraps and apples and water. I'd probably stay on practice then when my mum doesn't give me money then I don't feel the need to grab some cake and stuff."

\section{Autonomy}

Self-determination theory ${ }^{299}$ suggests that competence alone is not sufficient to achieve adherence to new behaviours, it must be accompanied by autonomy and relatedness. Distinct from its literal meaning of independence or self-reliance, autonomy is a characteristic of motivational quality. At one end of the motivational spectrum, controlled motivation, including external regulation and introjection, are behaviours that are motivated by external regulations, such as the rewards, punishments, approval and disapproval that are controlled by others 416. More autonomous forms of motivation include identified regulation (personally endorsing or identifying with the importance of a behaviour) and integrated regulation (not only identifying with a behaviour but also aligning it with other central values and lifestyle patterns). Controlled regulation (external and introjected) is unrelated to long-term adherence, while both forms of autonomous motivation (identified and integrated) are associated with enhanced maintenance of behaviour change ${ }^{416}$ and weight loss maintenance ${ }^{209}$.

During group meetings, autonomy was encouraged by allowing participants the choice to self-determine their behaviour, participants were encouraged to come up with their own exercise plans, dietary changes and set individual goals. Furthermore, they were provided with a rationale and reason for adopting the desired behaviour as opposed to prescribing behaviours or giving ultimatums, and self-regulation was promoted to prevent the reliance on external regulation. Although the current analysis does not determine the true source of participants motivations, a more controlling environment in which children's behaviour is 
directed from the perspective of an adult rather than the child, may serve to undermine the experience of autonomy. Participant D remained heavily dependent on her mother for motivation and relied on feedback from study personnel, suggesting her motivation was regulated by external factors.

Parent D: "There are days that we need to give her a good shove to do the activity we have got planned but once she gets going she is ok. I motivate her, I keep her on track, I pick up the little sneaky treats (it's fruit mum) no it's not."

Parent D: "She wants to say that she has done it [her goals] and please you two [research staff] and keep it going, she likes having people who can see what she's done".

In contrast, participant A was highly independent in his behaviours and was not reliant on study personnel for feedback, suggesting a more autonomous motivation. The SDT also suggests that the goals individuals pursue can have intrinsic or extrinsic qualities which influence behaviour maintenance ${ }^{209}$. While it is possible participant A's motivation was introjected (preserving self-esteem and avoiding guilt), his goals relate to sporting aspirations, self-improvement and social interaction which are more self-determined forms of regulation and are associated with greater adherence ${ }^{221}$. Conversely, Participant D frequently referred to extrinsic goals related to physical appearance.

Participant D: "My goals were to achieve a super model body and to look good in a bikini"

While participant B was also reliant on his Grandma for much of his behaviour, later in the study, he gained independence acting on his own volition in a way that was not apparent for participant D. He played sport socially with friends and regulated screen time, unbeknown to his Grandma, suggesting less external control and more intrinsic goals.

Participant B: "My friends might call me some days, text me if I want to go out, text me are you awake? Do you want to play today? I tell my mum if I'm going out to play football with my friend."

These apparent differences in autonomy may offer some explanation for the varied response between participants. 
In accordance with the SDT, relatedness, a sense of belonging with others, is essential for autonomous motivation and behaviour maintenance ${ }^{299}$. During the group meetings, open discussion to share ideas and give feedback was designed to allow children to become affiliated with their peers. Moreover, a buddy system was introduced to monitor each other's goals and provide support between meetings. Despite efforts to promote peer social support within the study, previous research has suggested social support comes primarily from parents and very close friends, with classmates offering the least social support 417 . Indeed, parents are hugely influential as role models and providers of food and opportunities to be active/inactive ${ }^{418}$. It is therefore no surprise that parental involvement is an important component of effective interventions, especially in younger children ${ }^{32}$. In general, social support was encouraged by prompting individuals to draw on support from other people to help them achieve their target behaviour. Given the age of the participants this was almost exclusively their parents and the support varied according to their family dynamic. Interviews revealed that all participants frequently ate meals as a family, modelling healthy eating habits. Although this was the case for participant B while he stayed with his Grandma, the social influence from his mother was not supportive of healthy behaviour. He was unable to keep to his dietary goals while staying with his mother due to the food she provided and behaviours she modelled.

Parent B: "Food temptations comes from mum. No activity encouragement from mum".

Social support for physical activity was also somewhat varied, participants B and D performed physical activity primarily with their parents or guardian, participants A and C did physical activity with their friends. Participant A's parents were physically active themselves acting as role models while in contrast participant C's were not.

Participant A: "My dad is my role model in an active way because he played rugby and he encouraged me a lot to play sports and he played for the All Whites St. Helens and he played against New Zealand.”

Parent C: "My husband and I are pretty much not that active so we are not that fit so I'm afraid he takes after both of us." 
Participants $\mathrm{A}, \mathrm{B}$ and $\mathrm{C}$ referred to their friends as a positive influence encouraging them to engage in physical activity.

Participant B: "My friends might call me some days, text me if I want to go out, text me are you awake? Do you want to play today?"

Participant B: "looking at my friends and see how fit and healthy they are, I couldn't do half the things they could do, they would probably find it easy, so I wanna be like them,"

However, they also offer a source of temptation or distraction.

Parent C: "I think it's mixed with the exercise, some of them [friends] are more active than others, quite a few of them are computer enthusiasts so yeah that's one of the things he would like to do."

Research has shown that peers can positively influence overweight children's energy intake and food selection ${ }^{419}$. However, in the present study, a common theme was that friends represented a temptation to eat unhealthy foods.

Participant C: "Walking past shops with money or seeing my friends when I have money, seeing my friends buy sweets and that it can make you want to buy things."

Parent D: "She struggles a bit I think, she's got a friend across the road and they go up the corner shop quite often, because her friends like to snack and munch on sweets and stuff and she finds that difficult to be put in that situation."

While friends appear to have a significant influence on the behaviour, it was common that children did not tell their friends about the study or their goals suggesting that friends may also be a source of shame or embarrassment.

Parent A: "I don't think he talks to his friends about it [project]"

Parent C: "That's a difficult question for me because I don't think he talks to his friends about it [the program] at all."

Given the varied social influences, eliciting social support was as much about recognising the social influences that could hinder the maintenance of healthy behaviour. The buddy system is often proposed to facilitate social support ${ }^{420}$. However, in the present study it was not well adopted by the participants. This may have been due to the differing age and social interests in this small study sample. The reluctance of the children to tell their friends about the program 
would also have prevented a buddy system being established outside of the study.

\subsection{Summary}

At some time during the two years, the intervention proved effective for three of the four participants. These participants showed improvements in dietary and physical activity behaviour, reductions in BMI z-score HOMA-IR and HbAlc. Two of these participants retained these changes including a $>9 \%$ reduction in BMI z-score at 24 months. Both participants believed they would maintain their behaviours describing it as a way of life. Having achieved an $8 \%$ reduction in BMI z-score at 12 months, participant D experienced a relapse and an $11 \%$ increase in BMI z-score at 24 months. Despite this relapse, participant D and her mother perceived the study to have had a positive influence. They believed they had learnt a lot and have made changes to their behaviour incorporating physical activity

Parent D: "I think she's learned the importance of exercise and routine. I think she has learned the importance of healthy eating and why you should be careful about what you are eating and how it affects you. So it's all good." "Yeah. We have made a lot of changes in all fairness and we still are going through those changes."

The intervention appeared to have the least impact on participant $\mathrm{C}$ whose objective outcome measures showed little change throughout the intervention, with the exception of a rise in physical activity at 24 months which saw him achieve target of $>60$ minutes of physical MVPA for the first time. In agreement with the objective measures, his mother had been doubtful that he had made many changes at 6 and 12 months. However, at 24 months she believed his attitude had changed.

Parent C: "He certainly has become more aware of food. He is thinking about what he eats, he is doing different sorts of exercise although whether that would have happened anyway I don't know."

Unfortunately, food diaries indicate that at 24 months he consumed chocolate cereal, biscuits, sugary soft drinks and desserts. These two foods contributed to a high intake of free sugars and suggest dietary goals were not being followed. 
The use of semi structured interviews added context to the objective outcome measures indicating that behavioural changes had been made when they were not always detected by the accelerometer and food diaries or in the absence of a reduction in BMI z-score, possibly due to compensation effects or seasonal changes. They also provided insight into the varied family structure and social influences and their differing response to intervention objectives such as vulnerability, self-efficacy and autonomy that may have impacted on the study outcomes.

The child and parent perceptions also gave an indication of compliance with the study objectives and acceptability of the behaviour change techniques. For example, goal setting techniques were considered useful by participants but planning goals in a structured manner waned through the study with more successful participants internalising their goals instead of keeping a written plan. Compliance with self-monitoring techniques was low and largely reliant on parents. The use of monitoring techniques more appealing to children may be advised to fulfil this aspect of the behaviour change process.

The current study has got some limitations. The small number of cases presented is a clear limitation and precludes the ability to extrapolate the findings of this study beyond the presented cases. The study was also unable to include data from the participants that dropped out in an intention to treat analysis. While the participants who dropped out experienced reductions in BMI z-score, their perceptions of the study are likely different from completers.

It was not possible to blind the participant to the purpose of the study, as such, there is a possibility of socially desirable responses to questions in the semi structured interview.

\subsection{Conclusion}

In conclusion, this collection of case studies showed that a critical research informed behaviour intervention can be effective in reducing risk factors for type 2 diabetes in children aged $10-12$ years. However, responses to behaviour change objectives varied supporting an individualized approach. Compliance to self-monitoring and written goal setting techniques was poor, the use of selfmonitoring techniques that appeal to children such as phone apps should be considered in future interventions. 


\subsection{Thesis Map}

\begin{tabular}{|c|c|c|}
\hline Study & Aims & Findings \\
\hline $\begin{array}{l}\text { 1. } \\
\text { Objectively measured } \\
\text { physical activity and } \\
\text { sedentary time are associated } \\
\text { with cardio-metabolic risk } \\
\text { factors in adults with pre- } \\
\text { diabetes: The PREVIEW } \\
\text { study }\end{array}$ & $\begin{array}{l}\text { To measure the relationship between } \\
\text { cardio-metabolic risk factors and } \\
\text { objectively measured physical activity } \\
\text { and sedentary time. } \\
\mathrm{H}_{0} \text { : The magnitude of the association } \\
\text { between physical activity and cardio } \\
\text { metabolic risk factors is equal to zero }\end{array}$ & $\begin{array}{l}\text { In adults with pre-diabetes, } \\
\text { objectively measured physical } \\
\text { activity and sedentary time were } \\
\text { associated with cardio-metabolic } \\
\text { risk markers. Total physical } \\
\text { activity was at least as strongly } \\
\text { associated with cardio-metabolic } \\
\text { risk markers as MVPA }\end{array}$ \\
\hline $\begin{array}{l}2 . \\
\text { Correlates of physical } \\
\text { activity in adults with pre- } \\
\text { diabetes from } 8 \text { countries: } \\
\text { the PREVIEW study. }\end{array}$ & $\begin{array}{l}\text { To assess the correlates of objectively } \\
\text { measured physical activity in adults } \\
\text { with prediabetes in eight countries and } \\
\text { to examine inter-country differences } \\
\text { with site-specific interactions. } \\
\mathrm{H}_{0} \text { : The magnitude of association } \\
\text { between psychosocial variables and } \\
\text { physical activity is equal to zero } \\
\mathrm{H}_{0} \text { : There is no difference between } \\
\text { country in the magnitude of association } \\
\text { between psychosocial variables and } \\
\text { physical activity. }\end{array}$ & $\begin{array}{l}\text { Only a small number of } \\
\text { modifiable factors were } \\
\text { associated with MVPA. Social } \\
\text { support may be of more } \\
\text { importance in older participants } \\
\text { and in the presence of high } \\
\text { inactivity temptations while } \\
\text { some correlates of MVPA were } \\
\text { country-specific (self-efficacy } \\
\text { and goal adjustment). }\end{array}$ \\
\hline $\begin{array}{l}3 . \\
\text { Lifestyle behaviours } \\
\text { associated with body fat } \\
\text { percent in 9-11-year-old } \\
\text { children, a population-based, } \\
\text { cross-sectional study: The } \\
\text { SportsLinx project. }\end{array}$ & $\begin{array}{l}\text { To investigate the associations between } \\
\text { body fat percent, and lifestyle } \\
\text { behaviours in primary school children } \\
\text { on a population level. Further, to } \\
\text { examine the consistency of these } \\
\text { associations over a } 10 \text {-year period } \\
\mathrm{H}_{0} \text { : The association between lifestyle } \\
\text { behaviours and body fat } \% \text { is equal to }\end{array}$ & $\begin{array}{l}\text { No significant change in } \\
\text { associations between behaviours } \\
\text { and body fat were found over a } \\
10 \text {-year period. Active transport } \\
\text { to school, sleep and } \\
\text { consumption of full fat milk } \\
\text { were negatively associated with } \\
\text { body fat. }\end{array}$ \\
\hline
\end{tabular}

zero.

$\mathrm{H}_{0}$ : There is no difference between years in the magnitude of association between lifestyle behaviours and body fat $\%$.

\section{4.}

The effectiveness of a child weight management intervention; a mixed methods investigation of child and parent's perceptions of the behaviour change process.
To report on the effectiveness of a weight management intervention aimed at producing sustained change in diet and physical activity behaviours and reduction of type 2 diabetes risk factors. To give the contextual, subjective experience of the participants and their parents on the theoretical framework of an intervention and how this may affect compliance with the program objectives.
This collection of case studies showed that a critical research informed behaviour intervention can be effective in reducing risk factors for type 2 diabetes in children aged 10-12 years. However, responses to behaviour change objectives varied with poor compliance to self-monitoring and written goal setting techniques. 


\section{Summary}

The backdrop for this thesis was two-fold. First, high rates of overweight and obesity among children in general, but also specifically in Swansea were identified in the Swanlinx program. The Swanlinx program measured health and fitness in primary school children and highlighted the need for suitable treatment pathways for overweight children. Second, Swansea University's involvement in the PREVIEW project; an international diabetes prevention program combining up-to-date physical activity, nutrition and behaviour change science in a lifestyle intervention. These two studies provided the context to assess the feasibility of running an intervention in Swansea as part an exploratory arm of the PREVIEW study, while also furthering our understanding of diet and physical activity behaviours that are the target of lifestyle interventions.

The literature in chapter 1 showed that type 2 diabetes can be delayed or prevented by lifestyle interventions which produce modest weight loss through changes in diet and physical activity. However, it is also apparent that many interventions produce only marginal changes in physical activity and that behaviour change and subsequent weight loss is rarely maintained. Our understanding of the role of physical activity in this population is hampered by the almost exclusive reliance on self-report questionnaires, which are prone to error, especially in overweight/obese populations. To address these gaps in the literature, this thesis began by scrutinizing the associations between physical activity and markers of cardio-metabolic health in a worldwide sample of adults with pre-diabetes.

In chapter 5, accelerometers were used to provide an objective measure of physical activity, sedentary time and sleep in participants of the PREVIEW intervention. This chapter showed that MVPA was negatively associated with insulin resistance (HOMA-IR), waist circumference, fasting insulin, 2-hour glucose, triglycerides and C-reactive protein. Furthermore, independent of time spent in MVPA, sedentary time was positively associated with the same cardiometabolic markers, in addition to systolic and diastolic blood pressure. A key finding of this study was that associations between total physical activity and all cardio-metabolic markers were at least as strong as with MVPA. While 
recognising the limitations of cross-sectional associations, the implications of this finding in a population that largely do not meet guidelines for MVPA, are that limiting sedentary time and the accumulation of total physical activity is as important as achieving the intensity of MVPA. Following these findings, I decided to identify the factors that facilitate participation in physical activity specific to this population. In chapter 6, we assessed the correlates of objectively-measured physical activity in adults with pre-diabetes in multiple countries and to examine inter-country differences. Novel features of this study were the use of an objective measure of physical activity but also the use of a standardised protocol across eight countries. This allowed us to examine the differences between countries. Two-way interactions showed that social support was of greater importance in the presence of inactivity temptations, i.e. with high inactivity temptations, social support averts a negative impact on physical activity. Similarly, social support may be of greater importance in older participants. These findings offer a better understanding of the role of social support, a frequent component of lifestyle interventions, and imply interventions may be improved if social support is a focus for older participants and at times of temptations to be inactive.

Central to the theory of planned behaviour ${ }^{229}$, is the tenet that intention is a key determinant of behaviour. Within lifestyle interventions, action-planning techniques are used to form an intention to engage in physical activity. In chapter 6, we showed that intention was positively associated with physical activity in adults with pre-diabetes. However, intention only predicts a small proportion of behaviour ${ }^{334}$, behaviours also occur as a habitual response to environment cues without volitional control or awareness ${ }^{335}$. These two constructs (intention and habit strength) have been shown to interact to determine behaviour ${ }^{335}$. While no interaction effect was found in chapter 6 , habit strength was negatively associated with physical activity. Thus, it may be pertinent for interventions to focus on habit strength in combination with intention to affect physical activity behaviours.

Other psychosocial variables, self-efficacy and goal adjustment, were only significantly associated with physical activity in some countries. While it remains unclear why these differences occurred from our analysis, future studies 
should consider the possibilities that some correlates of physical activity are country specific.

Despite those with pre-diabetes being at the highest risk of developing type 2 diabetes and representing the population at which many lifestyle interventions are targeted, few studies have been conducted in participants exclusively diagnosed with pre-diabetes. Therefore, the findings of chapter 5 and 6 provide evidence directly applicable to participants of intervention such as the NHS Diabetes Prevention Program (DPP) ${ }^{421}$. In the DPP, physical activity guidelines are to achieve gradual increase in physical activity up to 30 minutes of MVPA per day in line with the Chief Medical Officer's guidelines ${ }^{422}$. The findings in chapter 5 suggest total activity over the day, which could be achieved through light activity and reducing sedentary time, is as important as MVPA. Furthermore, social support is encouraged within the DPP through participants working together in group activities, setting group goals and engaging in social interaction during breaks ${ }^{423}$. Based on the finding of chapter 6 , this may be enhanced by including social support from family members for older adults and encouraging social support from friends to counteract inactivity temptations.

In chapter 7 and thereafter, this thesis focused on aspects of child interventions. Given that obesity is the greatest risk factor for type 2 diabetes, in chapter 7, key lifestyle behaviours were identified that were associated with body fat percent in children aged 9-12 years. Over the past 15 years screen based behaviour has changed, with time spent watching television decreasing and a sharp increase in computer use ${ }^{271}$. Likewise over the past decade there has been a year-on-year shift from full fat to skimmed milk varieties and an increase of wholemeal bread 272 and consumption of low calorie soft drinks ${ }^{274}$. Despite these changes, it was unclear whether lifestyle behaviours and food choices impact on adiposity over time. The pathogenic processes associated with obesity are related to body fat; BMI is only a composite measure using height and weight and is not a measure of body composition. Skinfold measures, on the other hand, allow a 2compartment model (fat and fat-free mass) to be determined. This study aimed to improve on previous literature by including a measure of body fat percent instead of BMI. The key findings of this study were that despite alterations in behaviour and the composition of foods over time, associations with childhood adiposity have not significantly changed over a 10 -year period. The behaviours 
that were negatively associated with body fat percent, were late bedtime, active forms of transport to school and full fat milk consumption. Low fat milk and dairy foods are often recommended as health alternatives to reduce energy density and saturated fat intake ${ }^{383}$. However, our finding that full fat milk was negatively associated with body fat percent joins other cross-sectional and prospective studies and literature reviews that call this recommendation into question $138,270,374,375,378,387$.

This study does not provide insight into the mechanisms behind this association but others have suggested bioactive effects of the fatty acids contained in milk which have been shown to have an effect on low grade inflammation, oxidative stress ${ }^{381}$ and insulin resistance ${ }^{138}$. Furthermore, the greater fat content may increase satiety and result in a net reduced calorie intake ${ }^{382}$. The findings of the present study add to this evidence by showing that at a population level, the consumption of full fat milk is negatively associated with body fat percent in 911 year old children. Randomised control trials have been conducted in this area, and conclude that dairy consumption can lead to weight reduction if combined with a calorie restricted diet ${ }^{424}$. However, most clinical trials have specified the intake of low fat dairy in line with nutritional guidelines. Low and full fat dairy foods have been shown to have conflicting associations with obesity which may have encumbered these findings ${ }^{375}$. Future interventions should compare low and full fat dairy consumptions with obesity outcomes.

Despite reports that sugar intake in the UK far exceeds recommended levels ${ }^{304}$ and evidence that sugar is linked to obesity and weight gain ${ }^{267}$, we found no association between the consumption of biscuits, cakes, chocolate bars, fizzy drinks or added sugar with body fat percent. We did find a counter intuitive negative association between sugary cereals and body fat percent. This finding agrees with outcomes from the NHANES study that found that, regardless of sugar content, children who ate cereal had significantly lower BMI compared with children who did not ${ }^{368}$. Furthermore, cereal consumption was associated with lower fat intake, a higher intake of carbohydrates, whole grains and a range of vitamins ${ }^{368}$. Similarly, in a recent study of Australian children, no associations were found between anthropometric measures and the choice of non-sweetened or sweetened breakfast cereal and added and free sugar intake was similar between non-cereal consumers and cereal consumers. On the other 
hand, cereal consumers had lower sodium and higher intake of total sugars, carbohydrate, dietary fibre, and almost all other micronutrients, irrespective of whether they consumed non-sweetened or sweetened breakfast cereal ${ }^{369}$. The strength of this study was its large sample size capturing associations between behaviour and body fat on a population level. However, the survey methods employed were unable to measure portion size or a complete nutrient intake profile, preventing more detailed analysis of the observed associations. Based on our findings and the wider literature, sugary cereals consumption does not appear to be indicative of a poor diet or a predictor of increased body fat. Consequently, broadly targeting sugary cereal intake during interventions may inadvertently affect the intake of important nutrients. Future studies should consider the wider nutritional profile of cereals products.

The final experimental study investigated the feasibility of running the PREVIEW intervention in a sub group of children in Swansea. This chapter provided an insight into the challenges of recruiting overweight and obese children into a lifestyle intervention, a process rarely reported in the literature. Valuable lessons were learnt for future intervention efforts. Having recruited only a small sample, it was not possible to make inferences regarding the effectiveness of the intervention or its subcomponents. Therefore, the decision was taken to describe the intervention process in a series of case studies combining the subjective experience of the participants and their parents with objective outcome measures in a mixed-methods design. This provided context to the behaviour change process, offered insight into compliance with the study objectives and how well the study was accepted by the participants. At some time during the 2 years, the intervention was effective for 3 of the 4 cases presented. These participants showed improvements in dietary and physical activity behaviour, reductions in BMI z-score HOMA-IR and HbA1c. Two of these participants retained these changes including a $>9 \%$ reduction in BMI zscore at 24 months.

The inclusion of semi-structured interviews in combination with objective outcome measures revealed that in some cases behavioural changes had occurred despite not always being detected by the accelerometers and food diaries, possibly due to compensation effects or seasonal changes. The semi-structured 
interviews also provided insight into the varied family structure and social influences that impact on diet and physical activity behaviours. These differences affect how each family interacted with the intervention and may explaining the variation of responses to the intervention. The child and parent perceptions demonstrated that goal setting techniques were considered useful although planning goals in a structured manner waned through the study with more successful participants internalising their goals as opposed to keeping a written plan. Compliance with self-monitoring techniques was low and largely reliant on parents. Self-monitoring is regarded as a key component to the behaviour change process. Therefore, poor compliance may have hindered the effectiveness of this intervention.

It is probable that perceptions about the intervention differed between participants who completed the intervention and those who dropped out. Therefore, the inability to include data from participants who dropped out, and the small number of cases presented, limits the external validity of these findings. However, there is an urgent need for treatment pathways for overweight and obese children. This study provides an insight into what an intervention may look like and how different intervention components were received by participants. Future interventions should look to develop selfmonitoring techniques that are appealing to children. These may include mobile phone apps, pedometers or accelerometers that automate the process as much as possible and reduce the need for written materials. Furthermore, future studies should, when possible, include data from participants who drop out of the intervention. This would provide a valuable insight into barriers to participation that may in turn improve the acceptability of future interventions.

This body of work has expanded the current knowledge base on the relationships between physical activity and cardio-metabolic health, by applying objective measures of physical activity and sedentary time in an international sample of adults with pre-diabetes. This thesis has shown empirically that total physical activity, MVPA and sedentary time are associated with cardio-metabolic health in pre-diabetics. Furthermore, the accumulation of total physical activity volume is as strongly associated with cardio-metabolic health as achieving the MVPA goals. Accumulating a high physical activity volume, can be achieved without 
achieving the intensity of MVPA. For example, by reducing sedentary time and increasing light activity. This may provide a practical approach to improve cardio-metabolic health in a population with low engagement in MVPA.

The ability for this study to define the associations between specific exercise intensities and cardio metabolic health, is limited by the confines of conventional regression techniques. The length of a day is fixed, therefore, if the day is divided into time spent in different activities (sleep, light activity sedentary time and MVPA), these subcomponents of a day are inherently collinear. Thus, the inclusion of all accelerometer-derived variables (sleep, light activity sedentary time and MVPA) in a single regression model would violate collinearity assumptions. Physical activity does not happen in insolation but is combined with sedentary periods, light activity and sleep. Further research should look to apply more sophisticated statistical techniques, such a compositional analysis, or isotemporal substitution, which can account for this intrinsic co-dependency ${ }^{425}$.

As the data used in this thesis were cross-sectional and the dependent variables were continuous, linear regression analysis was used extensively to measure the associations between the exposures of interest and the dependent variable, while controlling for confounders. In the first two studies, stepwise, multiple linear regression models were used with multiple imputation methods to account for missing data. In the third study, data were collected from 15,977 children attending 116 different schools. When data are collected from a hierarchical structure, observations can be correlated with one another. Conventional regression methods tend to underestimate standard errors in clustered data as they do not account for this correlated structure of observations. Consequently, there is a greater likelihood of type 1 error. Therefore, multilevel models were used to provide a more accurate assessment of the relationships between lifestyle behaviours and body fat percent, as they account for the clustering of children with schools. Possible limitations to this analysis include the absence of school level variables. While the dependent variable (body fat percentage) did vary by school, we were not able to include school level variables that may have explained this variance. Future research should look to combine individual and 
school level variables to examine the associations between school characteristics and individual behaviours that lead to obesity.

This thesis adds to the current evidence on correlates of physical activity by including an objective measure of physical activity and a standardised protocol across eight countries, strengthening the literature that up to now has largely uses self-report measures. Limitations of this study include its cross-sectional design which precludes inferences regarding causality. Correlates research attempts to predict why some people are active but others are not. Prospective studies provide causal links between psychosocial factors and physical activity but to my knowledge this is yet to be shown in adults with pre-diabetes. Stronger evidence is provided by interventions demonstrating a mediating effect between theoretical constructs and behaviour change. However, evidence of this nature is limited and results are mixed ${ }^{426}$. While correlates research provides useful evidence about possible mediators of physical activity for future interventions, the next step is to examine their causative role in intervention trials. Although psychosocial variables are central to behaviour change interventions, evidence of their mediating effect on physical activity is inconsistent and hindered by selfreport measures of physical activity. Future research is needed to address these issues and better define the role of factors that encourage physical activity. The child and parent perceptions of the lifestyle intervention described in this thesis, provides context to the techniques that aim to influence mediators of behaviour change. While this is not evidence of their effectiveness, it offers insight into their acceptability in children. There is an urgent need for effective treatment pathways for overweight and obese children and none are currently available in Swansea. This thesis has demonstrated that a research informed intervention can reduce risk factors for type 2 diabetes in overweight and obese children. 


\section{References}

1. Wang, Y. C., McPherson, K., Marsh, T., Gortmaker, S. L. \& Brown, M. Health and economic burden of the projected obesity trends in the USA and the UK. Lancet 378, 815-825 (2011).

2. GBD Obesity Collaborators. Health Effects of Overweight and Obesity in 195 Countries over 25 Years. N. Engl. J. Med. 377, 13-27 (2017).

3. Gregg, E. W. \& Shaw, J. E. Global Health Effects of Overweight and Obesity. N. Engl. J. Med. 377, 80-81 (2017).

4. World Health Organization. WHO | The top 10 causes of death. WHO (2017). Available at:

http://www.who.int/mediacentre/factsheets/fs310/en/. (Accessed: 27th July 2017)

5. Lee, I.-M. et al. Impact of Physical Inactivity on the World's Major NonCommunicable Diseases. Lancet 380, 219-229 (2012).

6. Duncan, G. E. et al. Exercise training, without weight loss, increases insulin sensitivity and postheparin plasma lipase activity in previously sedentary adults. Diabetes Care 26, 557-562 (2003).

7. Hu, F. B. Globalization of diabetes: The role of diet, lifestyle, and genes. Diabetes Care 34, 1249-1257 (2011).

8. Wiegand, S. et al. Type 2 diabetes and impaired glucose tolerance in European children and adolescents with obesity - a problem that is no longer restricted to minority groups. Eur. J. Endocrinol. 151, 199-206 (2004)

9. Dabelea, D. et al. Prevalence of Type 1 and Type 2 Diabetes among children and adolescents from 2001 to 2009. Jama 311, 1778-1786 (2014)

10. Haines, L., Chong Wan, K., Lynn, R., Barrett, T. G. \& Shield, J. Rising Incidence of Type 2 Diabetes in Children in the U.K. Diabetes Care 30, 1097-1101 (2007).

11. Hilier, T. A. \& Pedula, K. Complications in Young Adults With EarlyOnset Type 2 Diabetes. Diabetes Care 26, 2999-3005 (2003).

12. Liu, H. Association of Type 1 Diabetes vs Type 2 Diabetes Diagnosed During Childhood and Adolescence With Complications During Teenage Years and Young Adulthood. JAMA Psychiatry 317, 825-835 (2017).

13. Neil, A. Diabetes and cardiovascular disease. Diabetes, Obesity and Metabolism 5, S11-S18 (2016).

14. Yau, J. W. Y. et al. Global Prevalence and Major Risk Factors of Diabetic Retinopathy. Diabetes Care 35, 556-564 (2012).

15. Tuttle, K. R. et al. Diabetic Kidney Disease: A Report from an ADA Consensus Conference. Am. J. Kidney Dis. 64, 510-533 (2014).

16. Noto, H., Osame, K., Sasazuki, T. \& Noda, M. Substantially increased risk of cancer in patients with diabetes mellitus: A systematic review and meta-analysis of epidemiologic evidence in Japan. J. Diabetes Complications 24, 345-353 (2010).

17. Melton, J. G. International Diabetes Federation, Diabetes Atlas. (2017). Available at: http://www.diabetesatlas.org/resources/2017-atlas.html. 
(Accessed: 8th January 2018)

18. Hex, N., Bartlett, C., Wright, D., Taylor, M. \& Varley, D. Estimating the current and future costs of Type 1 and Type 2 diabetes in the UK, including direct health costs and indirect societal and productivity costs. Diabet. Med. 29, 855-862 (2012).

19. Zhang, P. et al. Global epidemiology of diabetic foot ulceration: a systematic review and meta-analysis $\dagger$. Ann. Med. 49, 106-116 (2017).

20. Diabetes Prevention Program Research Group. 10-year follow-up of diabetes incidence and weight loss in the Diabetes Prevention Program Outcomes Study. Lancet 374, 1677-1686 (2011).

21. Lindström, J. et al. Sustained reduction in the incidence of type 2 diabetes by lifestyle intervention: follow-up of the Finnish Diabetes Prevention Study. Lancet 368, 1673-9 (2006).

22. Li, G. et al. The long-term effect of lifestyle interventions to prevent diabetes in the China Da Qing Diabetes Prevention Study: a 20-year follow-up study. Lancet 371, 1783-9 (2008).

23. Curioni, C. C. \& Lourenço, P. M. Long-term weight loss after diet and exercise: A systematic review. Int. J. Obes. 29, 1168-1174 (2005).

24. Swift, D. L., Johannsen, N. M., Lavie, C. J., Earnest, C. P. \& Church, T. $\mathrm{S}$. The role of exercise and physical activity in weight loss and maintenance. Prog. Cardiovasc. Dis. 56, 441-447 (2014).

25. Hawley, J. A. Exercise as a therapeutic intervention for the prevention and treatment of insulin resistance. Diabetes. Metab. Res. Rev. 20, 383-393 (2004).

26. Pan, X. R. et al. Effects of diet and exercise in preventing NIDDM in people with impaired glucose tolerance: The Da Qing IGT and diabetes study. Diabetes Care 20, 537-544 (1997).

27. Rabinovich, R. A. et al. Validity of physical activity monitors during daily life in patients with COPD. Eur. Respir. J. 42, 1205-1215 (2013).

28. Shephard, R. J. Limits to the measurement of habitual physical activity by questionnaires. Br. J. Sports Med. 37, 197-206 (2003).

29. Warner, E. T. et al. Differential accuracy of physical activity self-report by body mass index. Am. J. Health Behav. 36, 168-178 (2012).

30. Tudor-Locke, C. E. \& Myers, A. M. Challenges and opportunities for measuring physical activity in sedentary adults. Sport. Med. 31, 91-100 (2001).

31. Ho, M. et al. Effectiveness of Lifestyle Interventions in Child Obesity: Systematic Review With Meta-analysis. Pediatrics 130, e1647-e1671 (2012).

32. Oude, L. et al. Interventions for treating obesity in children. Cochcrane Database Syst. Rev. CD001872, (2009).

33. Gow, M. L. et al. Can early weight loss, eating behaviors and socioeconomic factors predict successful weight loss at 12- and 24-months in adolescents with obesity and insulin resistance participating in a randomised controlled trial? Int. J. Behav. Nutr. Phys. Act. 13, 1 (2016).

34. Martin, J., Chater, A. \& Lorencatto, F. Effective behaviour change techniques in the prevention and management of childhood obesity. Int. J. Obes. 37, 1287-1294 (2013).

35. Katzmarzyk, P. T. et al. Relationship between lifestyle behaviors and obesity in children ages 9-11: Results from a 12-country study. Obesity 
23, 1696-1702 (2015).

36. Janssen, I. et al. Comparison of overweight and obesity prevalence in school-aged youth from 34 countries and their relationships with physical activity and dietary patterns. Obes. Rev. 6, 123-132 (2005).

37. Kahn, S. E., Cooper, M. E. \& Prato, S. Del. Pathophysiology and treatment of type 2 diabetes : perspectives on the past, present, and future. Lancet 383, 1068-1083 (2014).

38. Kaufman, F. R. Type 2 diabetes mellitus in children and youth: A new epidemic. J. Pediatr. Endocrinol. Metab. 15, 737-744 (2002).

39. Pinhas-Hamiel, O.\& Zeitler, P. The global spread of type 2 diabetes mellitus in children and adolescents. J. Pediatr. 146, 693-700 (2005).

40. Worlds Health Organization. Gaining health: The European strategy for the prevention and control of noncommunicable diseases. Copenhagen: World Health Organization (2006). Available at:

http://www.euro.who.int/_data/assets/pdf_file/0008/76526/E89306.pdf\% 5Cnhttp://scholar.google.com/scholar?hl=en\&btnG=Search\&q=intitle:Gai ning + Health + The + European + Strategy + for + the + Prevention+and + Control + of + Noncommunicable+Diseases\#0. (Accessed: 8th January 2018)

41. Center for Disease Control. National Diabetes Statistics Report: Estimates of Diabetes and Its Burden in the United States. US Department of Health and Human Services 2009-2012 (2017). Available at:

https://www.cdc.gov/diabetes/pdfs/data/statistics/national-diabetesstatistics-report.pdf. (Accessed: 8th January 2018)

42. DeFronzo, R. A. Lilly lecture 1987. The triumvirate: beta-cell, muscle, liver. A collusion responsible for NIDDM. Diabetes 37, 667-87 (1988).

43. Kahn, S. E. The relative contributions of insulin resistance and beta-cell dysfunction to the pathophysiology of Type 2 diabetes. Diabetologia 46, 3-19 (2003).

44. Pratley, R. E. \& Weyer, C. The role of impaired early insulin secretion in the pathogenesis of Type II diabetes mellitus. Diabetologia 44, 929-945 (2001).

45. Shaw, J. . et al. Impaired Fasting Glucose or Impaired Glucose Tolerance. Diabetes Care 22, (1999).

46. Weyer, C., Bogardus, C., Mott, D. M. \& Pratley, R. E. The natural history of insulin secretory dysfunction and insulin resistance in the pathogenesis of type 2 diabetes mellitus. J. Clin. Invest. 104, 787-794 (1999).

47. Gabir, M. M. et al. The 1997 American Diabetes Association and 1999 World Health Organization criteria for hyperglycemia in the diagnosis and prediction of diabetes. Diabetes Care 23, 1108-1112 (2000).

48. Færch, K., Borch-Johnsen, K., Holst, J. J. \& Vaag, A. Pathophysiology and aetiology of impaired fasting glycaemia and impaired glucose tolerance: Does it matter for prevention and treatment of type 2 diabetes? Diabetologia 52, 1714-1723 (2009).

49. Tabák, A. G., Herder, C., Rathmann, W., Brunner, E. J. \& Kivimäki, M. Prediabetes: a high-risk state for diabetes development. Lancet 379, 227990 (2012).

50. Tabák, A. G., Herder, C. \& Kivimäki, M. Prediabetes : A high-risk state for developing diabetes Progression from prediabetes to diabetes Reversion to normoglycaemia Risk prediction. Pmc 379, 1-14 (2017).

51. Jameson, J. L. et al. Endocrinology : adult \&amp; pediatric. (Elsevier, 
2016).

52. Diagnosis and classification of diabetes mellitus. Diabetes Care 34, (2011).

53. Manuscript, A. \& Mellitus, D. The implications of using Hemoglobin A1C for diagnosing Diabetes Mellitus. Am J Med 124, 395-401 (2012).

54. WHO/IDF. Definition and diagnosis of diabetes mellitus and intermediate hyperglycemia : report of a WHO/IDF consultation. (2006).

55. Ogurtsova, K. et al. IDF Diabetes Atlas: Global estimates for the prevalence of diabetes for 2015 and 2040. Diabetes Res. Clin. Pract. 128, 40-50 (2017).

56. Whiting, D. R., Guariguata, L., Weil, C. \& Shaw, J. IDF Diabetes Atlas: Global estimates of the prevalence of diabetes for 2011 and 2030.

Diabetes Res. Clin. Pract. 94, 311-321 (2011).

57. Guariguata, L. et al. Global estimates of diabetes prevalence for 2013 and projections for 2035. Diabetes Res. Clin. Pract. 103, 137-149 (2014).

58. NCD Risk Factor Collaboration. Worldwide trends in diabetes since 1980: a pooled analysis of 751 population-based studies with 4.4 million participants. Lancet (London, England) 387, 1513-1530 (2016).

59. Evans, J. M., Newton, R. W., Ruta, D. a, MacDonald, T. M. \& Morris, a D. Socio-economic status, obesity and prevalence of Type 1 and Type 2 diabetes mellitus. Diabet. Med. 17, 478-80 (2000).

60. Boyle, J., Engelgau, M., Thompson, T. \& Goldsmith, M. Estimating prevalence of type 1 and type 2 diabetes within a population of African Americans with diabetes mellitus. Diabetes 149, (1998).

61. Bruno, G. et al. Incidence of type 1 and type 2 diabetes in adults aged 3049 years: The population-based registry in the province of Turin, Italy. Diabetes Care 28, 2613-2619 (2005).

62. Hotu, S., Carter, B., Watson, P. D., Cutfield, W. S. \& Cundy, T. Increasing prevalence of type 2 diabetes in adolescents. $J$ Paediatr Child Heal. 40, 201-204 (2004).

63. McMahon, S. K. et al. Increase in type 2 diabetes in children and adolescents in Western Australia. Med. J. Aust. 180, 459-61 (2004).

64. Kiess, W. et al. Type 2 Diabetes mellitus in Children and Adolescents: A Review from a European Perspective. Horm. Res. Paediatr. 59, 77-84 (2004).

65. Wabitsch, M. et al. Type II diabetes mellitus and impaired glucose regulation in Caucasian children and adolescents with obesity living in Germany. Int. J. Obes. Relat. Metab. Disord. 28, 307-313 (2004).

66. Harris, S. B., Perkins, B. A. \& Whalen-Brough, E. Non-insulin-dependent diabetes mellitus among First Nations children: New entity among First Nations people of northwestern Ontario. Can. Fam. Physician 42, 869876 (1996).

67. Hsia, Y. et al. An increase in the prevalence of type 1 and 2 diabetes in children and adolescents: results from prescription data from a UK general practice database. Br. J. Clin. Pharmacol. 67, 242-9 (2009).

68. Ehtisham, S., Hattersley, A. T., Dunger, D. B. \& Barrett, T. G. First UK survey of paediatric type 2 diabetes and MODY. Arch. Dis. Child. 89, 526-529 (2004).

69. Ghergherechi, R. \& Tabrizi, A. Prevalence of impaired glucose tolerance and insulin resistance among obese children and adolescents. Ther. Clin. 
Risk Manag. 6, 345-349 (2010).

70. Sinha, R. et al. Prevalence of Impaired Glucose Tolerance Among Children and Adolescents with Marked Obesity. N. Engl. J. Med. 346, 802-810 (2002).

71. Fagot-Campagna, A., Saaddine, J., Fligal, K. M. \& Beckles, Gloria L, A. Diabetes, Impaired Fasting Glucose, and Elevated HbA1c in U.S. Adolescents: The Third National Health and Nutrition Examination Survey. Diabetes Care 24, 24-27 (2001).

72. Weiss, R. et al. Predictors of Changes in Glucose Tolerance Status in Obese Youth. Diabetes Care 28, 902-909 (2005).

73. Jaruratanasirikul, S., Thammaratchuchai, S., Puwanant, M., Mo-suwan, L. \& Sriplung, H. Progression from impaired glucose tolerance to type 2 diabetes in obese children and adolescents : a 3 - 6-year cohort study in southern Thailand. J. Pediatr. Endocrinol. Metab. (2016). doi:10.1515/jpem-2016-0195

74. World Health Organization. Global Report on Diabetes Executive Summary. (2016). Available at: http://apps.who.int/iris/bitstream/10665/204874/1/WHO_NMH_NVI_16.3 eng.pdf?ua=1. (Accessed: 8th January 2018)

75. Donnelly, R., Emslie-Smith, A. M., Gardner, I. D. \& Morris, A. D. ABC of arterial and venous disease: vascular complications of diabetes. Bmj 320, 1062-1066 (2000).

76. World Health Organization. WHO | The top 10 causes of death. World Health Organization (2013). Available at: http://www.who.int/mediacentre/factsheets/fs310/en/. (Accessed: 17th April 2014)

77. Nathan, D. Long-term complications of Diabetes Mellitus. N. Engl. J. Med. 328, 1676-1685 (1993).

78. Moxey, P. W. et al. Lower extremity amputations - a review of global variability in incidence. Diabet. Med. 28, 1144-1153 (2011).

79. Larsson, S. C., Mantzoros, C. S. \& Wolk, A. Diabetes mellitus and risk of breast cancer: A meta-analysis. Int. J. Cancer 121, 856-862 (2007).

80. Everhart, J. \& Wright, D. Diabetes mellitus as a risk factor for pancreatic cancer. A meta-analysis. Jama 273, 1605-1609 (1995).

81. El-Serag, H. B., Hampel, H. \& Javadi, F. The association between diabetes and hepatocellular carcinoma: A systematic review of epidemiologic evidence. Clin. Gastroenterol. Hepatol. 4, 369-380 (2006).

82. Zhuo, X., Zhang, P. \& Hoerger, T. J. Lifetime direct medical costs of treating type 2 diabetes and diabetic complications. Am. J. Prev. Med. 45, 253-261 (2013).

83. Petersen, M. Economic costs of diabetes in the U.S. in 2012. Diabetes Care 39, 1033-1046 (2016).

84. Saydah, S., Bullard, K. M., Imperatore, G., Geiss, L. \& Gregg, E. W. Cardiometabolic Risk Factors Among US Adolescents and Young Adults and Risk of Early Mortality. Pediatrics 131, e679-e686 (2013).

85. Rhodes, E. T. et al. Estimated morbidity and mortality in adolescents and young adults diagnosed with Type 2 diabetes mellitus. Diabet. Med. 29, 453-463 (2012).

86. Gale, E. A. M. \& Gillespie, K. M. Diabetes and gender. Diabetologia 44, 3-15 (2001). 
87. Meigs, J. B., Cupples, L. A. \& Wilson, P. W. F. Parental Transmission of Type 2 Diabetes The Framingham Offspring Study. World Health 57, 2201-2207 (2000).

88. Van 't Riet, E. et al. Role of Adiposity and Lifestyle in the Relationship Between Family. Diabetes Care 33, 763-767 (2010).

89. Sakurai, M. et al. Family history of diabetes, lifestyle factors, and the 7year incident risk of type 2 diabetes mellitus in middle-aged japanese men and women. J. Diabetes Investig. 4, 261-268 (2013).

90. Fuchsberger, C. et al. The genetic architecture of type 2 diabetes. Nature 536, 41-47 (2016).

91. Sladek, R. et al. A genome-wide association study identifies novel risk loci for type 2 diabetes. Nature 445, 881-885 (2007).

92. Zeggini, E. et al. Europe PMC Funders Group Meta-analysis of genomewide association data and large-scale replication identifies additional susceptibility loci for type 2 diabetes. Nat Genet 40, 638-645 (2009).

93. Bao, W. et al. Predicting risk of type 2 diabetes mellitus with genetic risk models on the basis of established genome-wide association markers: A systematic review. Am. J. Epidemiol. 178, 1197-1207 (2013).

94. Huang, T. et al. Genetic predisposition to central obesity and risk of type 2 diabetes: Two independent cohort studies. Diabetes Care 38, 1306-1311 (2015).

95. Qi, L., Cornelis, M. C., Zhang, C., Dam, R. M. Van \& Hu, F. B. Genetic predisposition, Western dietary pattern, and the risk of type 2 diabetes in men. Am. Soc. Nutr. 1-6 (2009). doi:10.3945/ajcn.2008.27249.Am

96. Karter, A. J. et al. Ethnic disparities in diabetic complications in an insured population. Jama 287, 2519-2527 (2002).

97. Shai, I. et al. Ethnicity, Obesity, and Risk of Type 2 Diabetes in Women: A 20-year follow-up study. Diabetes Care 29, 1585-1590 (2006).

98. Golden, S. H. et al. Health disparities in endocrine disorders: Biological, clinical, and nonclinical factors - An endocrine society scientific statement. J. Clin. Endocrinol. Metab. 97, (2012).

99. Choi, S. E., Liu, M., Palaniappan, L. P., Wang, E. J. \& Wong, N. D. Gender and ethnic differences in the prevalence of type 2 diabetes among Asian subgroups in California. J Diabetes Complicat. 27, 429-435 (2014).

100. Ardisson Korat, A. V., Willett, W. C. \& Hu, F. B. Diet, Lifestyle, and Genetic Risk Factors for Type 2 Diabetes: A Review fromthe Nurses' Health Study, Nurses'Health Study 2, and Health Professionals' FollowUp Study. Curr. Nutr. Rep. 3, 345-354 (2014).

101. Jia, W. P. et al. Epidemiological characteristics of diabetes mellitus and impaired glucose regulation in a Chinese adult population: the Shanghai Diabetes Studies, a cross-sectional 3-year follow-up study in Shanghai urban communities. Diabetologia 50, 286-292 (2007).

102. Defay, R. et al. Relationships between physical activity, obesity and diabetes mellitus in a French elderly population: the POLA study. Int. J. Obes. 25, 512-518 (2001).

103. Bell, J. A., Kivimaki, M. \& Hamer, M. Metabolically healthy obesity and risk of incident type 2 diabetes: A meta-analysis of prospective cohort studies. Obes. Rev. 15, 504-515 (2014).

104. Population, S. Diet, lifestyle, and the risk of type 2 diabetes mellitus in women. N. Engl. J. Med. 345, 790-797 (2001). 
105. Wang, Y., Rimm, E. B., Stampfer, M. J., Willett, W. C. \& Hu, F. B. Comparison of abdominal adiposity and overall obesity in predicting risk of type 2 diabetes among men. Am. J. Clin. Nutr. 81, 555-63 (2005).

106. Qiao, Q. \& Nyamdorj, R. Is the association of type II diabetes with waist circumference or waist-to-hip ratio stronger than that with body mass index. Eur. J. Clin. Nutr. 64, 30-34 (2010).

107. Papaetis, G. S., Papakyriakou, P. \& Panagiotou, T. N. Central obesity, type 2 diabetes and insulin: exploring a pathway full of thorns. Arch. Med. Sci. 3, 463-482 (2015).

108. Vazquez, G., Duval, S., Jacobs, D. R. \& Silventoinen, K. Comparison of body mass index, waist circumference, and waist/hip ratio in predicting incident diabetes: A meta-analysis. Epidemiol. Rev. 29, 115-128 (2007).

109. Resnick, H. E., Valsania, P., Halter, J. \& Lin, X. Relation of weight gain and weight loss on subsequent diabetes risk in overweight adults. $J$. Epidemiol. Community Heal. 8, 596-602 (2000).

110. Kilkkinen, A. et al. Prevention of type 2 diabetes in a primary health care setting. Interim results from the Greater Green Triangle (GGT) Diabetes Prevention Project. Diabetes Res. Clin. Pract. 76, 460-2 (2007).

111. De Mutsert, R., Sun, Q., Willett, W. C., Hu, F. B. \& Van Dam, R. M. Overweight in early adulthood, adult weight change, and risk of type 2 diabetes, cardiovascular diseases, and certain cancers in men: A cohort study. Am. J. Epidemiol. 179, 1353-1365 (2014).

112. Waring, M. E., Eaton, C. B., Lasater, T. M. \& Lapane, K. L. Incident diabetes in relation to weight patterns during middle age. Am. J. Epidemiol. 171, 550-556 (2010).

113. French, S. a et al. Weight variability and incident disease in older women: the Iowa Women's Health Study. Int. J. Obes. 21, 217-223 (1997).

114. Yokomichi, H. et al. Weight cycling and the subsequent onset of type 2 diabetes mellitus: 10-year cohort studies in urban and rural Japan. BMJ Open 7, 1-10 (2017).

115. Neamat-Allah, J. et al. Weight cycling and the risk of type 2 diabetes in the EPIC-Germany cohort. Diabetologia 58, 2718-2725 (2015).

116. Swinburn, B. a et al. The global obesity pandemic: shaped by global drivers and local environments. Lancet 378, 804-14 (2011).

117. Cho, S. S., Qi, L., Fahey, G. C. \& Klurfeld, D. M. Consumption of cereal fiber, mixtures of whole grain and bran, and whole grains and risk reduction in type 2 diabetes, obesity, and cardiovascular disease. Am J Clin Nutr 98, 594-619 (2013).

118. Bhupathiraju, S. N. et al. Glycemic index, glycemic load, and risk of type 2 diabetes: results from 3 large US cohorts and an updated meta-analysis. Am. J. Clin. Nutr. 100, 218-32 (2014).

119. Larsen, T. M. et al. Diets with High or Low Protein Content and Glycemic Index for Weight-Loss Maintenance. N. Engl. J. Med. 363, 2102-2113 (2012).

120. Hooper, L. et al. Effect of reducing total fat intake on body weight: systematic review and meta-analysis of randomised controlled trials and cohort studies. Bmj 345, e7666-e7666 (2012).

121. Riserus, Ulf, Willett, Walter C., Hu, F. B. Dietary fats and prevention of type 2 diabetes. Progress. Lipid Res. 48, 44-51 (2010).

122. Risérus, U. Fatty acids and insulin sensitivity. Curr. Opin. Clin. Nutr. 
Metab. Care 11, 100-105 (2008).

123. McEvoy, C. T. et al. A Posteriori Dietary Patterns Are Related to Risk of Type 2 Diabetes: Findings from a Systematic Review and Meta-Analysis. J. Acad. Nutr. Diet. 114, 1759-1775 (2014).

124. Pan, A., Sun, Q. \& Bernstein, A. Red meat consumption and risk of type 2 diabetes: 3 cohorts of US adults and an updated meta-analysis. Am. 1-9 (2011). doi:10.3945/ajen.111.018978

125. Malik, V. S. et al. Sugar-sweetened beverages and risk of metabolic syndrome and type 2 diabetes: a meta-analysis. Diabetes Care 33, 247781 (2010).

126. Imamura, F. et al. Consumption of sugar sweetened beverages, artificially sweetened beverages, and fruit juice and incidence of type 2 diabetes: systematic review, meta-analysis, and estimation of population attributable fraction. Bmj 351, h3576 (2015).

127. Greenwood, D. C. et al. Association between sugar-sweetened and artificially sweetened soft drinks and type 2 diabetes: systematic review and dose-response meta-analysis of prospective studies. Br. J. Nutr. 112, 725-734 (2014).

128. Li, M., Fan, Y., Zhang, X., Hou, W. \& Tang, Z. Fruit and vegetable intake and risk of type 2 diabetes mellitus: Meta-analysis of prospective cohort studies. BMJ Open 4, (2014).

129. Carter, P., Gray, L. J., Troughton, J., Khunti, K. \& Davies, M. J. Fruit and vegetable intake and incidence of type 2 diabetes mellitus: systematic review and meta-analysis. Bmj 341, c4229-c4229 (2010).

130. Wang, P.-Y., Fang, J.-C., Gao, Z.-H., Zhang, C. \& Xie, S.-Y. Higher intake of fruits, vegetables or their fiber reduces the risk of type 2 diabetes: A meta-analysis. J. Diabetes Investig. 7, 56-69 (2016).

131. Cooper, A. J., Forouhi, N. G. \& Ye, Z. Europe PMC Funders Group Fruit and vegetable intake and type 2 diabetes : EPIC-InterAct prospective study and meta-analysis. Eur J Clin Nutr 66, 1082-1092 (2013).

132. Li, S. et al. Fruit intake decreases risk of incident type 2 diabetes: an updated meta-analysis. Endocrine 48, 454-460 (2015).

133. Wu, Y., Zhang, D., Jiang, X. \& Jiang, W. Fruit and vegetable consumption and risk of type 2 diabetes mellitus: A dose-response metaanalysis of prospective cohort studies. Nutr. Metab. Cardiovasc. Dis. 25, 140-147 (2015).

134. de Souza, R. J. et al. Intake of saturated and trans unsaturated fatty acids and risk of all cause mortality, cardiovascular disease, and type 2 diabetes: systematic review and meta-analysis of observational studies. Bmj 351, 3978 (2015).

135. Schwab, U. et al. Effect of the amount and type of dietary fat on cardiometabolic risk factors and risk of developing type 2 diabetes, cardiovascular diseases, and cancer: a systematic review. Food Nutr. Res. 58, 25145 (2014).

136. Lindström, J. et al. High-fibre, low-fat diet predicts long-term weight loss and decreased type 2 diabetes risk: the Finnish Diabetes Prevention Study. Diabetologia 49, 912-20 (2006).

137. Tong, X., Dong, J. Y., Wu, Z. W., Li, W. \& Qin, L. Q. Dairy consumption and risk of type 2 diabetes mellitus: A meta-analysis of cohort studies. Eur. J. Clin. Nutr. 65, 1027-1031 (2011). 
138. Kratz, M., Baars, T. \& Guyenet, S. The relationship between high-fat dairy consumption and obesity, cardiovascular, and metabolic disease. Eur. J. Nutr. 52, 1-24 (2013).

139. Baliunas, D. O. et al. Alcohol as a risk factor for type 2 diabetes: A systematic review and meta-analysis. Diabetes Care 32, 2123-2132 (2009).

140. Knott, C., Bell, S. \& Britton, A. Alcohol consumption and the risk of type 2 diabetes: A systematic review and Dose-Response Meta-analysis of more than 1.9 million individuals from 38 observational studies. Diabetes Care 38, 1804-1812 (2015).

141. Ding, M., Bhupathiraju, S. N., Chen, M., Van Dam, R. M. \& Hu, F. B. Caffeinated and decaffeinated coffee consumption and risk of type 2 diabetes: A systematicreview and a dose-response meta-analysis. Diabetes Care 37, 569-586 (2014).

142. Yang, J., Mao, Q.-X., Xu, H.-X., Ma, X. \& Zeng, C.-Y. Tea consumption and risk of type 2 diabetes mellitus: a systematic review and meta-analysis update. BMJ Open 4, e005632 (2014).

143. Huxley, R., Man, C., Lee, Y., Barzi, F. \& Timmermeister, L. Coffee, Decaffeinated Coffee, and Tea Consumption in Relation to Incident Type 2 Diabetes Mellitus. 169, 2053-2063 (2014).

144. Jannasch, F., Kroger, J. \& Schulze, M. Dietary Patterns and Type 2 Diabetes: A Systematic Literature Review and Meta-Analysis of Prospective Studies. J. Nutr. 147, 1174-1182 (2017).

145. Cloostermans, L. et al. Independent and combined effects of physical activity and body mass index on the development of Type 2 Diabetes - a meta-analysis of 9 prospective cohort studies. Int. J. Behav. Nutr. Phys. Act. 12, 147 (2015).

146. Aune, D., Norat, T., Leitzmann, M., Tonstad, S. \& Vatten, L. J. Physical activity and the risk of type 2 diabetes: A systematic review and doseresponse meta-analysis. Eur. J. Epidemiol. 30, 529-542 (2015).

147. Borghouts, L. B., Backx, K., Mensink, M. F. \& Keizer, H. A. Effect of training intensity on insulin sensitivity as evaluated by insulin tolerance test. Eur. J. Appl. Physiol. Occup. Physiol. 80, 461-466 (1999).

148. Henson, J. et al. Associations of objectively measured sedentary behaviour and physical activity with markers of cardiometabolic health. Diabetologia 56, 1012-1020 (2013).

149. Fedewa, M. V., Gist, N. H., Evans, E. M. \& Dishman, R. K. Exercise and Insulin Resistance in Youth: A Meta-Analysis. Pediatrics 133, e163-e174 (2014).

150. Holloszy, J. O. Exercise-induced increase in muscle insulin sensitivity. $J$. Appl. Physiol. 99, 338-43 (2005).

151. Plasqui, G. \& Westerterp, K. R. Physical Activity and Insulin Resistance. Curr. Nutr. Food Sci. 3, 157-160 (2007).

152. Villegas, R. et al. Physical activity and the incidence of type 2 diabetes in the Shanghai women's health study. Int. J. Epidemiol. 35, 1553-1562 (2006).

153. Weinstein, A. R. Relationship of Physical Activity vs Body Mass Index With Type 2 Diabetes in Women. Jama 292, 1188 (2004).

154. Ainsworth, B. E. et al. 2011 compendium of physical activities: A second update of codes and MET values. Med. Sci. Sports Exerc. 43, 1575-1581 
(2011).

155. Smith, A. D., Crippa, A., Woodcock, J. \& Brage, S. Physical activity and incident type 2 diabetes mellitus: a systematic review and dose-response meta-analysis of prospective cohort studies. Diabetologia 59, 2527-2545 (2016).

156. Laaksonen, D. E. et al. Physical Activity in the Prevention of Type 2 Diabetes. Br. J. Nutr. 83 Suppl 1, S137-42 (2005).

157. Demakakos, P., Hamer, M., Stamatakis, E. \& Steptoe, A. Low-intensity physical activity is associated with reduced risk of incident type 2 diabetes in older adults: Evidence from the English Longitudinal Study of Ageing. Diabetologia 53, 1877-1885 (2010).

158. Gando, Y. et al. Light-Intensity Physical Activity Is Associated with Insulin Resistance in Elderly Japanese Women Independent of Moderateto Vigorous-Intensity Physical Activity. J. Phys. Act. Heal. 11, 266-271 (2014).

159. Mayer-Davis, E. . et al. Intensity and Amount of Physical Activity in Relation to Insulin Sensitivity: The Insulin Resistance Atherosclerosis Study. J. Am. Med. Assoc. 279, 669-674 (1998).

160. Houmard, J. A. et al. Effect of the volume and intensity of exercise training on insulin sensitivity on insulin sensitivity. 27858, 101-106 (2011).

161. Jelleyman, C. et al. The effects of high-intensity interval training on glucose regulation and insulin resistance: A meta-analysis. Obes. Rev. 16, 942-961 (2015).

162. Dempsey, P. C., Owen, N., Biddle, S. J. H. \& Dunstan, D. W. Managing Sedentary Behavior to Reduce the Risk of Diabetes and Cardiovascular Disease. Curr Diab Rep 14, (2014).

163. Chau, J. Y. et al. Daily Sitting Time and All-Cause Mortality: A MetaAnalysis. 8, 1-14 (2013).

164. Biswas, A. et al. Sedentary Time and Its Association With Risk for Disease Incidence , Mortality, and Hospitalization in Adults A Systematic Review and Meta-analysis. (2015). doi:10.7326/M14-1651

165. Cooper, A. R. et al. Sedentary time, breaks in sedentary time and metabolic variables in people with newly diagnosed type 2 diabetes. Diabetologia 55, 589-599 (2012).

166. Megeney, L. A., Neufer, P. D. \& G.L., D. Effects of muscle activity ad fiber composition on glucose transport and GLUT4. Am J Clin Nutr 264, E583-593 (1993).

167. Bey, L. \& Hamilton, M. T. Suppression of skeletal muscle lipoprotein lipase activity during physical inactivity : a molecular reason to maintain daily low-intensity activity. 673-682 (2003). doi:10.1113/jphysiol.2003.045591

168. Thaman, R. \& Arora, G. Metabolic Syndrome: Definition and Pathophysiology- the discussion goes on! J. Physiol. Pharmacol. Adv. 3, 48 (2013).

169. O'Neill, S. \& O'Driscoll, L. Metabolic syndrome: A closer look at the growing epidemic and its associated pathologies. Obes. Rev. 16, 1-12 (2015).

170. Mottillo, S. et al. The metabolic syndrome and cardiovascular risk: A systematic review and meta-analysis. J. Am. Coll. Cardiol. 56, 1113-1132 
(2010).

171. Ford, E. S. Risks for all-cause mortality, cardiovascular disease, and diabetes associated with the metabolic syndrome: A summary of the evidence. Diabetes Care 28, 1769-1778 (2005).

172. Alberti, K. G. M. M., Zimmet, P. \& Shaw, J. Metabolic syndrome-a new world-wide definition. A Consensus Statement from the International Diabetes Federation. Diabet. Med. 23, 469-480 (2006).

173. Christian K.Roberts, Andrea L.Hevener, and R. J. B. Metabolic Syndrome and Insulin Resistance: Underlying Causes and Modification by Exercise Training. Compr Physiol 3, 1-58 (2014).

174. Haffner, S. M. et al. Prospective analysis of the insulin-resistance syndrome (syndrome X). Diabetes 41, 715-22 (1992).

175. Nesto, R. W. The relation of insulin resistance syndromes to risk of cardiovascular disease. Rev. Cardiovasc. Med. 4 Suppl 6, S11-8 (2003).

176. Kaur, J. A comprehensive review on metabolic syndrome. Cardiol. Res. Pract. 2014, (2014).

177. Ekelund, U., Brage, S., Griffin, S. J. \& Wareham, N. J. Objectively Measured Moderate- and Vigorous-Intensity Physical Activity but Resistance in High-Risk Individuals. Diabetes Care 32, 1081-1086 (2009).

178. Rosique-Esteban, N. et al. Leisure-time physical activity, sedentary behaviors, sleep, and cardiometabolic risk factors at baseline in the PREDIMED-PLUS intervention trial: A cross-sectional analysis. PLoS One 12, e0172253 (2017).

179. Prince, S. et al. A comparison of direct versus self-report measures for assessing physical activity in adults: a systematic review. Int. J. Behav. Nutr. Phys. Act. 5, 56 (2008).

180. Welk, G. J. Principles of design and analyses for the calibration of accelerometry-based activity monitors. Med. Sci. Sports Exerc. 37, 501$511(2005)$.

181. Watson, K. B. et al. Comparison of accelerometer cut points to estimate physical activity in US adults. 32, 660-69 (2015).

182. Byrom, B. \& Rowe, D. A. Measuring free-living physical activity in COPD patients: deriving methodology standards for clinical trials through a review of research studies. Contemp. Clin. Trials 47, 172-184 (2016).

183. Trost, S. G., Loprinzi, P. D., Moore, R. \& Pfeiffer, K. a. Comparison of accelerometer cut points for predicting activity intensity in youth. Med. Sci. Sports Exerc. 43, 1360-1368 (2011).

184. Loprinzi, P. D. et al. The Relationship of Actigraph Accelerometer CutPoints for Estimating Physical Activity With Selected Health Outcomes The Relationship of Actigraph Accelerometer Cut-Points. 1367, (2013).

185. Hall, K., Howe, C., Rana, S., Martin, C. \& Morey, M. METs and Accelerometry of Walking in Older Adults. Med. Sci. Sport. Exerc. 0-10 (2012). doi:10.1249/MSS.0b013e318276c73c

186. Whitcher, L. \& Papadopoulos, C. Accelerometer Derived Activity Counts and Oxygen Consumption between Young and Older Individuals. J. Aging Res. 2014, 8 (2014).

187. Hibbert, J. M., Broemeling, L. D., Isenberg, J. N. \& Wolfe, R. R. Determinants of free-living energy expenditure in normal weight and obese women measured by doubly labeled water. Obes Res 2, 44-53 
(1994).

188. Healy, G. N. et al. Objectively measured sedentary time, physical activity, and metabolic risk. Diabetes Care 31, 369-711. Healy GN, Wijndaele K, Dunstan DW, Shaw J (2008).

189. Nelson, R. K. et al. Daily physical activity predicts degree of insulin resistance: a cross-sectional observational study using the 2003-2004 National Health and Nutrition Examination Survey. Int. J. Behav. Nutr. Phys. Act. 10, 10 (2013).

190. Knaeps, S. et al. Independent Associations between Sedentary Time, Moderate-To-Vigorous Physical Activity, Cardiorespiratory Fitness and Cardio-Metabolic Health: A Cross-Sectional Study. PLoS One 11, 1-13 (2016).

191. Ekelund, U., Griffin, S. J. \& Wareham, N. J. Physical activity and metabolic risk in individuals with a family history of type 2 diabetes. Diabetes Care 30, 337-342 (2007).

192. Hamasaki, H. et al. Daily Physical Activity Assessed by a Triaxial Accelerometer Is Beneficially Associated with Waist Circumference, Serum Triglycerides, and Insulin Resistance in Japanese Patients with Prediabetes or Untreated Early Type 2 Diabetes. J. Diabetes Res. 2015, 526201 (2015).

193. Morrato, E. H., Hill, J. O., Wyatt, H. R., Ghushchyan, V. \& Sullivan, P. W. Physical activity in U.S. adults with diabetes and at risk for developing diabetes, 2003. Diabetes Care 30, 203-209 (2007).

194. Yates, T., Khunti, K., Bull, F., Gorely, T. \& Davies, M. J. The role of physical activity in the management of impaired glucose tolerance: A systematic review. Diabetologia 50, 1116-1126 (2007).

195. Tuomilehto J., Indstrom J., Eriksson J., Valle T., H. E. \& U. M. Prevention of Type 2 Diabetes Mellitus By Changes in Lifestyle Among Subjects With Impaired Glucose Tolerance. N. Engl. J. Med. 344, 13431350 (2001).

196. Hamman, R. F. et al. Effect of weight loss with lifestyle intervention on risk of diabetes. Diabetes Care 29, 2102-2107 (2006).

197. Bauman, A. E. et al. Correlates of physical activity: Why are some people physically active and others not? Lancet 380, 258-271 (2012).

198. Trost, S. G., Owen, N., Bauman, A. E., Sallis, J. F. \& Brown, W. Correlates of adults' participation in physical activity: review and update. Med. Sci. Sport. Exerc. 34, 1996-2001 (2002).

199. Sallis, J. ., Owen, N. \& Fisher, E, B. Health Behaviour and Health Education, ECOLOGICAL MODELS OF HEALTH BEHAVIOR. (JosseyBass, 2014). doi:10.1146/annurev-psych-010213-115123

200. van Stralen, M. M., de Vries, H., Mudde, A. N., Bolman, C. \& Lechner, L. Determinants of initiation and maintenance of physical activity among older adults: A literature review. Health Psychol. Rev. 3, 147-207 (2009).

201. Blanchard, C. et al. Social ecological correlates of physical activity in normal weight, overweight, and obese individuals. Int. J. Obes. 29, 720726 (2005).

202. Van Dyck, D. et al. Interacting psychosocial and environmental correlates of leisure-time physical activity: A three-country study. Heal. Psychol 33, 699-709 (2014).

203. Bourdeaudhuij, I. De, Teixeira, P. J., Cardon, G. \& Deforche, B. 
Environmental and psychosocial correlates of physical activity in Portuguese and Belgian adults. Public Health Nutr. 8, (2005).

204. Jurj, A. L. et al. Patterns and correlates of physical activity: a crosssectional study in urban Chinese women. BMC Public Health 7, 213 (2007).

205. Chen, Y.-J. et al. The correlates of leisure time physical activity among an adults population from southern Taiwan. BMC Public Health 11, 427 (2011).

206. Luszczynska, A., Gutiérrez-Doña, B. \& Schwarzer, R. General selfefficacy in various domains of human functioning: Evidence from five countries. Int. J. Psychol. 40, 80-89 (2005).

207. Luszczynska, A., Gibbons, F. X., Piko, B. F. \& Tekozel, M. Selfregulatory cognitions, social comparison, and perceived peers' behaviors as predictors of nutrition and physical activity: A comparison among adolescents in Hungary, Poland, Turkey, and USA. Psychol. Heal. 19, 577-593 (2004).

208. Wu, T.-Y., Pender, N. \& Yang, K.-P. Promoting physical activity among Taiwanese and American adolescents. The Journal Of Nursing Research: JNR 10, 57-64 (2002).

209. Silva, M. N., Teixeira, P. J. \& Santos, I. A self-determination theory perspective on weight loss maintenance. Eur. Heal. Psychol. 18, 194-199 (2016).

210. Donahue, K. E. et al. Identifying Supports and Barriers to Physical Activity in Patients at Risk for Diabetes. Prev. Chronic Dis. 3, A119 (2006).

211. Delahanty, L. M., Conroy, M. B. \& Nathan, D. M. Psychological Predictors of Physical Activity in the Diabetes Prevention Program. J. Am. Diet. Assoc. 106, 698-705 (2006).

212. Janiszewski, P. M. P., Ross, R., Care, C., Janiszewski, P. M. P. \& Ross, R. Effects of Weight Loss Among Metabolically Healthy Obese Men. Diabetes Care 33, 1957-1959 (2010).

213. Glanz, K. \& Bishop, D. B. The Role of Behavioral Science Theory in Development and Implementation of Public Health Interventions. Annu. Rev. Public Health 31, 399-418 (2010).

214. Teixeira, P. J. et al. Successful behavior change in obesity interventions in adults: A systematic review of self-regulation mediators. BMC Med. 13, 1-16 (2015).

215. Prochaska, J. O. \& Velicer, W. F. The Transtheoretical Change Model of Health Behavior. Am. J. Heal. Promot. 12, 38-48 (1997).

216. Dallow, C. B. \& Anderson, J. Using self-efficacy and a transtheoretical model to develop a physical activity intervention for obese women. Am. J. Heal. Promot. 17, 373-381 (2003).

217. Finckenor, M. \& Byrd-Bredbenner, C. Nutrition intervention group program based on preaction-stage-oriented change processes of the Transtheoretical Model promotes long-term reduction in dietary fat intake. Journal of the American Dietetic Association 100, 335-342 (2000).

218. Naa, T. et al. Transtheoretical model for dietary and physical exercise modification in weight loss management for overweight and obese adults ( Review ). Prim. Care 2-4 (2012). doi:10.1002/14651858.CD008066.pub2.Copyright 
219. Mastellos, N., LH, G., LM, F., Car, J. \& Majeed, A. Transtheoretical model stages of change for dietary and physical exercise modification in weight loss management for overweight and obese adults. Cochrane Database Syst. Rev. 2, 8066 (2014).

220. Ryan, R. M., Patrick, H., Deci, E. L. \& Williams, G. C. Facilitating health behaviour change and its maintenance : Interventions based on SelfDetermination Theory. (2007).

221. Ingledew, D. K., Markland, D. \& Ferguson, E. Three Levels of Exercise Motivation. Appl. Psychol. Heal. Well-Being 1, 336-355 (2009).

222. Trief, P. M., Cibula, D., Delahanty, L. M. \& Weinstock, R. S. Selfdetermination theory and weight loss in a Diabetes Prevention Program translation trial. J. Behav. Med. 40, 483-493 (2017).

223. Hwang, J. \& Kim, Y. H. Physical activity and its related motivational attributes in adolescents with different BMI. Int. J. Behav. Med. 20, 106113 (2013).

224. Weiner, B. An Attributional Theory of Achievement Motivation and Emotion. Am. Psychol. Assoc. 92, 548-573 (1985).

225. McVay, M. A., Steinberg, D. M., Askew, S., Kaphingst, K. A. \& Bennett, G. G. Genetic causal attributions for weight status and weight loss during a behavioral weight gain prevention intervention. Genet. Med. 18, 476482 (2016).

226. Wamsteker, E. W., Geenen, R., Zelissen, P. M. J., van Furth, E. F. \& Iestra, J. Unrealistic Weight-Loss Goals among Obese Patients Are Associated with Age and Causal Attributions. J. Am. Diet. Assoc. 109, 1903-1908 (2009).

227. Bandura, A. Health promotion by social cognitive means. Heal. Educ. Behav. 31, 143-164 (2004).

228. Bandura, A. Health promotion from the perspective of social cognitive theory. Psychol. Heal. 13, 623-649 (1998).

229. Ajzen, I. The theory of planned behavior. Orgnizational Behav. Hum. Decis. Process. 50, 179-211 (1991).

230. Conner, M. \& Armitage, C. J. Extending the Theory of Planned Behavior: A Review and Avenues for Further Research \n. J. Appl. Soc. Psychol. 28, 1429-1464 (1998).

231. Stadler, G., Oettingen, G. \& Gollwitzer, P. M. Intervention Effects of Information and Self-Regulation on Eating Fruits and Vegetables Over Two Years. Heal. Psychol. 29, 274-283 (2010).

232. Hall, P. A., Zehr, C., Paulitzki, J. \& Rhodes, R. Implementation Intentions for Physical Activity Behavior in Older Adult Women: An Examination of Executive Function as a Moderator of Treatment Effects. Ann. Behav. Med. 48, 130-136 (2014).

233. Daddario, D. K. A review of the use of the health belief model for weight management. MEDSURG Nurs. 16, 363-366 4p (2007).

234. Baranowski, T., Cullen, K. W., Nicklas, T., Thompson, D. \& Baranowski, J. Are Current Health Behavioral Change Models Helpful in Guiding Prevention of Weight Gain Efforts? Obes. Res. 11, 23S-43S (2003).

235. Michie, S. \& Abraham, C. Daily episodes of worrying and stressors increase daytime- and nighttime cardiac activity. Psychol. Heal. 19, 29 (2004).

236. Prestwich, A. et al. Does Theory Influence the Effectiveness of Health 
Behavior Interventions? Meta-Analysis. Heal. Psychol. 33, 465-474 (2013).

237. Davies, P., Walker, A. E. \& Grimshaw, J. M. A systematic review of the use of theory in the design of guideline dissemination and implementation strategies and interpretation of the results of rigorous evaluations. Implement Sci 5, 14 (2010).

238. Michie, S., Stralen, M. M. Van \& West, R. The behaviour change wheel : A new method for characterising and designing behaviour change interventions The behaviour change wheel: A new method for characterising and designing behaviour change interventions. Implement. Sci. 42, (2011).

239. Greaves, C. J. et al. Systematic review of reviews of intervention components associated with increased effectiveness in dietary and physical activity interventions. BMC Public Health 11, 119 (2011).

240. Samdal, G. B., Eide, G. E., Barth, T., Williams, G. \& Meland, E. Effective behaviour change techniques for physical activity and healthy eating in overweight and obese adults; systematic review and meta-regression analyses. Int. J. Behav. Nutr. Phys. Act. 14, 1-14 (2017).

241. Golley, R. K., Hendrie, G. A., Slater, A. \& Corsini, N. Interventions that involve parents to improve children's weight-related nutrition intake and activity patterns - what nutrition and activity targets and behaviour change techniques are associated with intervention effectiveness? Obes. Rev. 12, 114-130 (2011).

242. Li, R. et al. Economic evaluation of combined diet and physical activity promotion programs to prevent type 2 diabetes among persons at increased risk: A systematic review for the community preventive services task force. Ann. Intern. Med. 163, 452-460 (2015).

243. Ramachandran, A. et al. The Indian Diabetes Prevention Programme shows that lifestyle modification and metformin prevent type 2 diabetes in Asian Indian subjects with impaired glucose tolerance (IDPP-1). Diabetologia 49, 289-297 (2006).

244. Kosaka, K., Noda, M. \& Kuzuya, T. Prevention of type 2 diabetes by lifestyle intervention: A Japanese trial in IGT males. Diabetes Res. Clin. Pract. 67, 152-162 (2005).

245. Wen, B. et al. Reduction in The Incidence of Type 2 Diabetes with Lifestyle Intervention or Metformin. N Engl J Med 7, 393-404 (2002).

246. Knowler, W. C. et al. 10-year follow-up of diabetes incidence and weight loss in the Diabetes Prevention Program Outcomes Study. Lancet 374, 1677-86 (2009).

247. Davies, M. J. et al. Effectiveness of the diabetes education and self management for ongoing and newly diagnosed (DESMOND) programme for people with newly diagnosed type 2 diabetes: cluster randomised controlled trial. BMJ 336, 491-5 (2008).

248. Realf, K. \& Dallosso, H. Effectiveness of a diabetes education and self management programme ( DESMOND ) for people with newly diagnosed type 2 diabetes mellitus : three year follow-up of a cluster randomised controlled trial in primary care. BMJ Br. Med. J. 2333, 1-12 (2012).

249. Yates, T., Davies, M., Gorley, T., Bull, F. \& Khunti, K. Effectiveness of a Pragmatic Education Program Designed to Promote Walking Activity in Individuals With Impaired Glucose Tolerance. Diabetes Care 32, 1404- 
1410 (2009).

250. Yates, T., Davies, M. J., Sehmi, S., Gorely, T. \& Khunti, K. The Prediabetes Risk Education and Physical Activity Recommendation and Encouragement (PREPARE) programme study: Are improvements in glucose regulation sustained at 2years? Diabet. Med. 28, 1268-1271 (2011).

251. Yates, T. et al. Walking Away from Type 2 diabetes: a cluster randomized controlled trial. Diabet. Med. 34, 698-707 (2017).

252. Davies, M. J. et al. A community based primary prevention programme for type 2 diabetes integrating identification and lifestyle intervention for prevention: The Let's Prevent Diabetes cluster randomised controlled trial. Prev. Med. (Baltim). 84, 48-56 (2016).

253. Lindstrom, J. et al. The Finnish Diabetes Prevention Study (DPS). Diabetes Care 26, 3230-3236 (2003).

254. Oldroyd, J. C., Unwin, N. C., White, M., Mathers, J. C. \& Alberti, K. G. M. M. Randomised controlled trial evaluating lifestyle interventions in people with impaired glucose tolerance. (2005). doi:10.1016/j.diabres.2005.09.018

255. Colberg, S. R. et al. Physical activity/exercise and diabetes: A position statement of the American Diabetes Association. Diabetes Care 39, 2065 2079 (2016).

256. Haskell, W. L. et al. Physical activity and public health: Updated recommendation for adults from the American College of Sports Medicine and the American Heart Association. Med. Sci. Sports Exerc. 39, 1423 1434 (2007).

257. U.S. Department of Health and Human Services. Physical Activity and Health: A Report of the Surgen General. Rev. Prat. 60, 1996 (1996).

258. Korkiakangas, E. E., Alahuhta, M. A. \& Laitinen, J. H. Barriers to regular exercise among adults at high risk or diagnosed with type 2 diabetes: A systematic review. Health Promot. Int. 24, 416-427 (2009).

259. Daniels, S. Complications of obesity in children and adolescents. Int. J. Obes. 33, 60-65 (2009).

260. Raitakari, O. T., Juonala, M. \& Kahonen, M. Cardiovascular risk factors in childhood and carotid artery intima-media thickness in adulthood. Jama 290, 2277-2283 (2003).

261. Summerbell, C. et al. Interventions for preventing obesity in children. Cochrane Database Syst. Rev. (2011). doi:10.1002/14651858.CD001871.pub3

262. Waters, E. \& de Silva-Sanigorski, A. Interventions for preventing obesity in children (review). Cochrane Database Syst. Rev. 2011-2014 (2012). doi:10.1002/14651858.CD001871

263. Liao, Y., Liao, J., Durand, C. P. \& Dunton, G. F. Which type of sedentary behaviour intervention is more effective at reducing body mass index in children? A meta-analytic review. Obes. Rev. 15, 159-168 (2014).

264. Janicke, D. M. et al. Systematic review and meta-analysis of comprehensive behavioral family lifestyle interventions addressing pediatric obesity. J. Pediatr. Psychol. 39, 809-825 (2014).

265. Eloranta, A.-M. et al. Dietary factors associated with overweight and body adiposity in Finnish children aged 6-8 years: the PANIC Study. Int. J. Obes. 36, 950-955 (2012). 
266. Noel, S. E., Ness, A. R., Northstone, K., Emmett, P. \& Newby, P. K. Milk Intakes Are Not Associated with Percent Body Fat in Children from Ages 10 to 13 Years. J. Nutr. 141, 2035-2041 (2011).

267. Morenga, L. ., Mallard, S. \& Mann, J. Dietary sugars and body weight: systematic review and meta-analyses of randomised controlled trials and cohort studies. Bmj 346, e7492-e7492 (2012).

268. Wilkie, H. J., Standage, M., Gillison, F. B., Cumming, S. P. \& Katzmarzyk, P. T. Multiple lifestyle behaviours and overweight and obesity among children aged 9-11 years: results from the UK site of the International Study of Childhood Obesity, Lifestyle and the Environment. BMJ Open 6, e010677 (2016).

269. Basterfield, L. et al. Physical activity, diet and BMI in children aged 6-8 years: a cross-sectional analysis. BMJ Open 4, e005001-e005001 (2014).

270. Holmberg, S. \& Thelin, A. High dairy fat intake related to less central obesity: a male cohort study with 12 years' follow-up. Scand. J. Prim. Health Care 31, 89-94 (2013).

271. WHO. Adolescent obesity and related behaviours : trends and inequalities in the WHO region 2002-2014. Who 2002-2014 (2017). Available at: http://www.euro.who.int/_data/assets/pdf_file/0019/339211/WHO_Obesi tyReport_2017_v3.pdf. (Accessed: 2nd February 2018)

272. DEFRA. Family Food 2014. (2015). Available at: https://www.gov.uk/government/uploads/system/uploads/attachment_data /file/485982/familyfood-2014report-17dec15.pdf.

273. Boddy, L. M., Abayomi, J., Johnson, B., Hackett, A. F. \& Stratton, G. Ten-year changes in positive and negative marker food, fruit, vegetables, and salad intake in 9-10 year olds: SportsLinx 2000-2001 to 2010-2011.J. Hum. Nutr. Diet. 27, 236-241 (2014).

274. BSDA, C. R. Children's Consumption of Soft Drinks. (2008). Available at: http://www.britishsoftdrinks.com/write/MediaUploads/Publications/BSD A_Consumer_Research_2008_Children's_Consumption_of_Soft_Drinks_(final).pdf.

275. Wing, R. R. \& Phelan, S. Long-term weight loss maintenance. Am. J. Clin. Nutr. 82, 34 (2005).

276. Fogelholm, M. et al. PREVIEW: Prevention of diabetes through lifestyle intervention and population studies in Europe and around the world. design, methods, and baseline participant description of an adult cohort enrolled into a three-year randomised clinical trial. Nutrients 9, (2017).

277. Lindström, J. \& Tuomilehto, J. The diabetes risk score: A practical tool to predict type 2 diabetes risk. Diabetes Care 26, 725-731 (2003).

278. Friedewald, W. T., Levy, R. I. \& Fredrickson, D. S. Estimation of the concentration of low-density lipoprotein cholesterol in plasma, without use of the preparative ultracentrifuge. Clin. Chem. 18, 499-502 (1972).

279. Wallace, T. M., Levy, J. C. \& Matthews, D. R. Use and abuse of HOMA modeling. Diabetes Care 27, 1487-1495 (2004).

280. Troiano, R. P. et al. Physical activity in the United States measured by accelerometer. Med. Sci. Sports Exerc. 40, 181-188 (2008).

281. Gabriel, K. P. et al. Issues in accelerometer methodology: The role of epoch length on estimates of physical activity and relationships with health outcomes in overweight, post-menopausal women. Int. J. Behav. 
Nutr. Phys. Act. 7, 1-10 (2010).

282. Tudor-locke, C., Barreira, T. V, Schuna, J. M., Mire, E. \& Katzmarzyk, P. T. Fully automated waist - worn accelerometer algorithm for detecting children's sleep period time separate from 24 - hour physical activity or sedentary behaviors. Appl. Physiol. Nutr. Metab. 39, 53-57 (2014).

283. Miller, G. D. et al. Effect of varying accelerometry criteria on physical activity: The look ahead study. Obesity 21, 32-44 (2013).

284. Oliver, M., Badland, H. M., Schofield, G. M. \& Shepherd, J. Identification of accelerometer nonwear time and sedentary behavior. Res. Q. Exerc. Sport 82, 779-783 (2011).

285. Renner, B. \& Schwarzer, R. Risk and Health Behaviors - Documentation of the Scales of the Research Project: "Risk Appraisal Consequences in Korea" (RACK). Int. Univ. Bremen Freie Univ. Berlin 27-28 (2005).

286. Schwarzer, R. \& Renner, B. Health-Specific Self-Efficacy Scales. Statistics (Ber). 5th Octobe, 1-21 (2009).

287. Wood, W., Tam, L. \& Witt, M. G. Changing Circumstances, Disrupting Habits. J. Pers. Soc. Psychol. 88, 918-933 (2005).

288. Wrosch, C., Scheier, M. F., Miller, G. E., Schulz, R. \& Carver, C. S. Adaptive Self-Regulation of Unattainable Goals: Goal Disengagement, Goal Reengagement, and Subjective Well-Being. Pers Soc Psychol Bull 29, 1494-1508 (2003).

289. Brubaker, B. An Attributional Analysis Of Weight Outcomes. Nurs Res 37, 282-287 (1988).

290. Hausenblas, H. A. et al. A missing piece of the transtheoretical model applied to exercise: Development and validation of the temptation to not exercise scale. Psychol. Health 16, 381-390 (2001).

291. Sallis, J. F., Grossman, R. M., Pinski, R. B., Patterson, T. L. \& Nader, P. $\mathrm{R}$. The development of scales to measure social support for diet and exercise behaviors. Prev. Med. (Baltim). 16, 825-836 (1987).

292. Donahue, K. E. et al. Identifying Supports and Barriers to Physical Activity in Patients at Risk for Diabetes. Prev. Chronic Dis. 3, A119 (2006).

293. Cole, T. J. \& Lobstein, T. Extended international (IOTF) body mass index cut-offs for thinness, overweight and obesity. Pediatr. Obes. 7, 284-294 (2012).

294. FAO \& WHO. Energy and Protein Requirements. Report of a joint FAO/WHO/UNU Expert Consultation. World Health Organ. Tech. Rep. Ser. 724, 1-206 (1985).

295. Department of Health and Social Care (Institution or Organization). Physical activity guidelines for Children and Young People. (2011). Available at:

https://www.gov.uk/government/uploads/system/uploads/attachment_data /file/213739/dh_128144.pdf. (Accessed: 2nd February 2018)

296. Kahlert, D., Unyi-reicherz, A., Stratton, G. \& Larsen, T. M. PREVIEW Behavior Modification Intervention Toolbox ( PREMIT ): A Study Protocol for a Psychological Element of a Multicenter Project. 7, (2016).

297. McGlothlin, H. \& Killen, M. When planning is not enough: Fighting unhealthy snacking habits by mental contrasting with implementation intentions. Eur. J. Soc. Psychol. Eur. 40, 625-634 (2010).

298. Strychar, I., Elisha, B. \& Schmitz, N. Type 2 diabetes self-management: 
Role of diet self-efficacy. Can. J. Diabetes 36, 337-344 (2012).

299. Deci, E. L. \& Ryan, R. M. The " What " and " Why " of Goal Pursuits : Human Needs and the Self-Determination of Behavior. Psychol. Inq. 11, 37-41 (2000).

300. Uwaifo, G. et al. Indices of Insulin Action, Disposal, and Secretion Derived From Fasting Samples and Clamps in Normal Glucose-Tolerant Black and White Children. Diabetes Care 25, 2081-2087 (2002).

301. Tudor-Locke, C. et al. Improving wear time compliance with a 24-hour waist-worn accelerometer protocol in the International Study of Childhood Obesity, Lifestyle and the Environment (ISCOLE). Int. J. Behav. Nutr. Phys. Act. 12, 11 (2015).

302. Esliger, D. W., Copeland, J. L., Barnes, J. D. \& Tremblay, M. S. Standardizing and optimizing the use of accelerometer data for free-living physical activity monitoring. J. Phys. Act. Heal. 2, 366-383 (2005).

303. Trost, S. G., Loprinzi, P. D., Moore, R. \& Pfeiffer, K. a. Comparison of accelerometer cut points for predicting activity intensity in youth. Med. Sci. Sports Exerc. 43, 1360-1368 (2011).

304. Bates, B. et al. National Diet and Nutrition Survey: Results from Years 1, 2, 3 and 4 (combined) of the Rolling Programme. 4, 1-27 (2014).

305. Taylor, S; Hackett, A; Stratton, G; Lamb, L. SportsLinx: Improving the Health and Fitness of Liverpool's Youth. Education and Health 22, 11-14 (2004).

306. Tremblay, M. et al. Systematic review of sedentary behaviour and health indicators in school-aged children and youth. Int. J. Behav. Nutr. Phys. Act. 8, 1-22 (2011).

307. Johnson, B., Hackett, A., Roundfield, M. \& Coufopoulos, A. An investigation of the validity and reliability of a food intake questionnaire. J. Hum. Nutr. Diet. 14, 457-465 (2001).

308. Hackett, A., Gibbon, M., Sratton, G. \& Hamill, L. Dietary intake of 9-10year-old and 11-12-year-old children in Liverpool. Public Health Nutr. 5, 449-455 (2007).

309. Index of Multiple Deprivation (IMD) 2007 - Datasets. Available at: https://data.gov.uk/dataset/index_of_multiple_deprivation_imd_2007. (Accessed: 21st July 2017)

310. Lohman, T. G., Roche, A. F. \& Martorell, R. Anthropometric standardization reference manual. Hum. Kinet. Books 177 (1988). doi:10.1002/ajhb.1310040323

311. Tanner, J. M. \& Whitehouse, R. H. Revised standards for triceps and subscapular skinfolds in British children. Arch Dis Child 50, 142-145 (1975).

312. Slaughter, M. H. et al. in Climate Change 2013 - The Physical Science Basis (ed. Intergovernmental Panel on Climate Change) 60, 1-30 (Cambridge University Press, 1988).

313. Léger, L. A., Mercier, D., Gadoury, C. \& Lambert, J. The multistage 20 metre shuttle run test for aerobic fitness The multistage 20 metre shuttle run test for aerobic fitness. J. Sports Sci. 6, 93-101 (1988).

314. Swindell, N. et al. Objectively Measured Physical Activity and Sedentary Time Are Associated With Cardiometabolic Risk Factors in Adults With Prediabetes: The PREVIEW Study. Diabetes Care 41, dc171057 (2017).

315. Silventoinen, K. et al. The validity of the Finnish Diabetes Risk Score for 
the prediction of the incidence of coronary heart disease and stroke, and total mortality. Eur. J. Cardiovasc. Prev. Rehabil. 12, 451-458 (2005).

316. Sterne, J. A. C. et al. Multiple imputation for missing data in epidemiological and clinical research: potential and pitfalls. BMJ 338, (2009).

317. Ford, E. S., Li, C. \& Zhao, G. Prevalence and correlates of metabolic syndrome based on a harmonious definition among adults in the US. $J$. Diabetes 2, 180-193 (2010).

318. Knutson, K. L. Sleep duration and cardiometabolic risk: a review of the epidemiologic evidence. Best Pr. Res. Clin. Endocrinol. Metab. 24, 731743 (2010).

319. Field, A. Discovering Statistics Using SPSS. International Statistical Review 81, (2013).

320. WHO. Global recommendations on physical activity for health. (2010).

321. Balkau, B., Mhamdi, L., Oppert, J. \& Nolan, J. Physical activity and insulin sensitivity the RISC study. Diabetes 57, 2613-2618 (2008).

322. Maher, C., Olds, T., Mire, E. \& Katzmarzyk, P. T. Reconsidering the sedentary behaviour paradigm. PLoS One 9, (2014).

323. Peddie, M. C. et al. Breaking prolonged sitting reduces postprandial glycemia in healthy, normal-weight adults: A randomized crossover trial. Am. J. Clin. Nutr. 98, 358-366 (2013).

324. Dempsey, P. C., Owen, N., Yates, T. E., Kingwell, B. A. \& Dunstan, D. W. Sitting Less and Moving More: Improved Glycaemic Control for Type 2 Diabetes Prevention and Management. Curr. Diab. Rep. 16, (2016).

325. Dempsey, P. et al. Interrupting prolonged sitting reduces resting blood pressure in adults with type 2 diabetes. Hear. Lung Circ. 24, S127-S128 (2015).

326. Richardson, E., Zaletel, J. \& Nolte, E. National Diabetes Plans in Europe What lessons are there for the prevention and control of chronic diseases in Europe? Available at: http://www.euro.who.int/_data/assets/pdf_file/0009/307494/Nationaldiabetes-plans-Europe.pdf. (Accessed: 6th December 2016)

327. International Diabetes Federation. Global Diabetes Plan 2011-2021. Vasa 1-20 (2011). Available at: https://www.idf.org/our-activities/advocacyawareness/resources-and-tools/129-global-diabetes-plan-2011-2021.html. (Accessed: 2nd February 2018)

328. Paulweber, B. et al. A European Evidence-Based Guideline for the Prevention of Type 2 Diabetes. 42, 3-36 (2010).

329. Chatterton, H., Younger, T., Fischer, A. \& Khunti, K. Risk identification and interventions to prevent type 2 diabetes in adults at high risk: summary of NICE guidance. Br. Med. J. 345, e4624-e4624 (2012).

330. Welk, G. Physical Activity Assessments for Health-related Research. 1, (Human Kinetics, 2002).

331. Van Dyck, D. et al. Environmental and psychosocial correlates of accelerometer-assessed and self-reported physical activity in Belgian adults. Int. J. Behav. Med. 18, 235-245 (2011).

332. Catellier, D. J. et al. Imputation of Missing Data When Measuring Physical Activity by Accelerometry. Med. Sci. Sport. Exerc. 37, S555S562 (2005).

333. Aiken, L. S., West, S. G. \& Reno, R. R. Multiple regression : testing and 
interpreting interactions. (Sage Publications, 1991).

334. Armitage, C. J. \& Conner, M. Efficacy of the Theory of Planned

Behaviour: A meta-analytic review. Br. J. Soc. Psychol. 40, 471-499 (2001).

335. Gardner, B. A Systematic Review and Meta-analysis of Applications of the Self-Report Habit Index to Nutrition and Physical Activity Behaviours. Ann. Behav. Med. 174-187 (2018). doi:10.1007/s 12160-0119282-0

336. McAuley, E., Szabo, A., Goth, N. \& Olson, E. Self-efficacy: Implications for Physical Activity, Function, and Functional Limitations in Older Adults. Am J Lifestyle Med 5, 1-15 (2011).

337. Sternfeld B.;Quesenberry, C., B. ;Ainswort. Physical activity patterns in a diverse population of women. Prev. Med. An Int. J. Devoted to Pract. Theory 28, 313-323 (1999).

338. Steptoe, A. et al. Leisure-time physical exercise: prevalence, attitudinal correlates, and behavioral correlates among young Europeans from 21 countries. Prev. Med. (Baltim). 26, 845-854 (1997).

339. Zunft, H. J. et al. Perceived benefits and barriers to physical activity in a nationally representative sample in the European Union. Public Health Nutr. 2, 153-160 (1999).

340. Sallis, J. F. et al. Neighborhood Environments and Physical Activity Among Adults in 11 Countries. Am. J. Prev. Med. 36, 484-490 (2009).

341. Wrosch, C., Miller, G. E., Scheier, M. F. \& de Pontet, S. B. Giving up on unattainable goals: Benefits for health? Personal. Soc. Psychol. Bull. 33, 251-265 (2007).

342. Wrosch, C. \& Sabiston, C. M. Goal Adjustment, Physical and Sedentary Activity, and Quality of Life Among Breast Cancer Survivors. PsychoOncology. 589, 581-589 (2013).

343. Avenell, A. et al. Systematic review of the long-term effects and economic consequences of treatments for obesity and implications for health improvement. Health Technol. Assess. (Rockv). 8, (2004).

344. Conolly, A. \& Neave, A. Health Survey for England 2015 Children 's body mass index , overweight and obesity. NHS Digitial 1-18 (2016). Available at: https://digital.nhs.uk/catalogue/PUB22610. (Accessed: 2nd February 2018)

345. Mizokami-Stout, K., Cree-Green, M. \& Nadeau, K. J. Insulin resistance in type 2 diabetic youth. Curr. Opin. Endocrinol. Diabetes. Obes. 19, 25562 (2012).

346. Pischon, T. et al. General and Abdominal Adiposity and Risk of Death in Europe. N. Engl. J. Med. 359, 2105-2120 (2008).

347. Kipping, R. R., Jago, R. \& Lawlor, D. A. Obesity in children. Part 1: Epidemiology, measurement, risk factors, and screening. Bmj 337, a1824a1824 (2008).

348. Fairclough, S. J., Boddy, L. M., Hackett, A. F. \& Stratton, G. Associations between children's socioeconomic status, weight status, and sex, with screen-based sedentary behaviours and sport participation. Int. J. Pediatr. Obes. 4, 299-305 (2009).

349. Plewis, I. \& Fielding, A. What is Multi-Level Modelling for? A Critical Response to Gorard (2003). Br. J. Educ. Stud. 51, 408-419 (2003).

350. Twisk, J. W. R. Applied multilevel analysis : a practical guide. 
(Cambridge University Press, 2006).

doi:10.1017/CBO9781107415324.004

351. Carpenter, J. J. R., Goldstein, H. \& Kenward, M. G. M. REALCOMIMPUTE Software for Multilevel Multiple Imputation with Mixed

Response Types. J. Stat. Softw. 45, 1-12 (2011).

352. Marshall, A., Altman, D. G., Holder, R. L. \& Royston, P. Combining estimates of interest in prognostic modelling studies after multiple imputation: Current practice and guidelines. BMC Med. Res. Methodol. 9, $1-8(2009)$.

353. Hart, C. N., Cairns, A. \& Jelalian, E. Sleep and Obesity in Children and Adolescents. Pediatr Clin North Am 58, 715-733 (2012).

354. Liu, J., Zhang, A. \& Li, L. Sleep duration and overweight/obesity in children: review and implications for pediatric nursing. J. Spec. Pediatr. Nurs. 17, 193-204 (2012).

355. Westerlund, L., Ray, C. \& Roos, E. Associations between sleeping habits and food consumption patterns among 10-11-year-old children in Finland. Br. J. Nutr. 102, 1531 (2009).

356. Padez, C., Mourao, I., Moreira, P. \& Rosado, V. Long sleep duration and childhood overweight/obesity and body fat. Am. J. Hum. Biol. 21, 371376 (2009).

357. Chen, M.-Y., Wang, E. K. \& Jeng, Y.-J. Adequate sleep among adolescents is positively associated with health status and health-related behaviors. BMC Public Health 6, 59 (2006).

358. Faulkner, G. E. J., Buliung, R. N., Flora, P. K. \& Fusco, C. Active school transport, physical activity levels and body weight of children and youth: A systematic review. Prev. Med. (Baltim). 48, 3-8 (2009).

359. Sullivan, J., Kershaw, K. \& Cummings, J. National Travel Survey: England 2015 Section. (2016). Available at:

https://www.gov.uk/government/uploads/system/uploads/attachment_data /file/551437/national-travel-survey-2015.pdf. (Accessed: 2nd February 2018)

360. Heitmann, B. L. \& Lissner, L. Dietary underreporting by obese individuals-is it specific or non-specific? BMJ Br. Med. J. 311, 986-989 (1995).

361. Giammattei, J., Blix, G., Marshak, H. H., Wollitzer, A. O. \& Pettitt, D. J. Television Watching and Soft Drink Consumption. Arch. Pediatr. Adolesc. Med. 157, 882 (2003).

362. Fowler, S. P. et al. Fueling the Obesity Epidemic? Artificially Sweetened Beverage Use and Long-term Weight Gain. Obesity 16, 1894-1900 (2008).

363. Kelly, B. et al. Examining opportunities for promotion of healthy eating at children's sports clubs. Aust. N. Z. J. Public Health 34, 583-588 (2010).

364. Mattes, R. D. \& Popkin, B. M. Nonnutritive sweetener consumption in humans: Effects on appetite and food intake and their putative mechanisms. Am. J. Clin. Nutr. 89, 1-14 (2009).

365. Duffey, K. J., Steffen, L. M., Horn, L. Van, Jacobs, D. R. \& Popkin, B. M. Dietary patterns matter: diet beverages and cardiometabolic risks in the longitudinal Coronary Artery Risk Development in Young Adults (CARDIA) Study 1-3. Am J Clin Nutr 95, 909-15 (2012).

366. Chandon, P. How package design and packaged-based marketing claims 
lead to overeating. Appl. Econ. Perspect. Policy 35, 7-31 (2013).

367. Woods, S. C. Signals that influence food intake and body weight. Physiol. Behav. 86, 709-716 (2005).

368. Albertson, A. M., Thompson, D. R., Franko, D. L. \& Holschuh, N. M. Weight indicators and nutrient intake in children and adolescents do not vary by sugar content in ready-to-eat cereal: Results from National Health and Nutrition Examination Survey 2001-2006. Nutr. Res. 31, 229-236 (2011).

369. Fayet-Moore, F., McConnell, A., Tuck, K. \& Petocz, P. Breakfast and breakfast cereal choice and its impact on nutrient and sugar intakes and anthropometric measures among a nationally representative sample of Australian children and adolescents. Nutrients 9, (2017).

370. Thomas, D., Elliott, E. J. \& Blaur, L. Low glycaemic index , or low glycaemic load, diets for diabetes mellitus ( Review ) Low glycaemic index, or low glycaemic load, diets for diabetes mellitus. Cochrane database Syst. Rev. 1-3 (2009). doi:10.1002/14651858.CD006296.pub2.Copyright

371. Shrapnel, B. Amount of sugar in Australian breakfast cereals is not associated with energy density or glycaemic index: Results of a systematic survey. Nutr. Diet. 70, 236-240 (2013).

372. Brennan, M. A., Derbyshire, E., Tiwari, B. K. \& Brennan, C. S. Ready-toeat snack products: The role of extrusion technology in developing consumer acceptable and nutritious snacks. Int. J. Food Sci. Technol. 48, 893-902 (2013).

373. Novotny, R., Daida, Y. G., Acharya, S., Grove, J. S. \& Voght, T. M. Diary Intake Is Associated with Lower Body Fat and Soda Intake with Greater Weight in Adolescent Girls. J. Nutr. 134, 1905-1909 (2004).

374. Crichton, G. E. \& Alkerwi, A. Whole-fat dairy food intake is inversely associated with obesity prevalence: Findings from the Observation of Cardiovascular Risk Factors in Luxembourg study. Nutr. Res. 34, 936943 (2014).

375. Berkey, C. S., Rockett, H. R. H., Willett, W. C. \& Colditz, G. A. Milk, Dairy Fat, Dietary Calcium, and Weight Gain. Arch. Pediatr. Adolesc. Med. 159, 543 (2005).

376. Harnack, L., Stang, J. \& Story, M. Soft Drink Consumption Amung US Children and Adolescents: Nutritional Consequences. J. Am. Diet Assoc. 99, 436-41 (1999).

377. Mrdjenovic, G. \& Levitsky, D. A. Nutritional and energetic consequences of sweetened drink consumption in 6- to 13-year-old children. J. Pediatr. 142, 604-610 (2003).

378. Beck, A. L., Heyman, M., Chao, C. \& Wojcicki, J. Full fat milk consumption protects against severe childhood obesity in Latinos. Prev. Med. Reports 8, 1-5 (2017).

379. Rosell, M., Håkansson, N. N. \& Wolk, A. Association between dairy food consumption and weight change over 9 y in 19,352 perimenopausal women. Am. J. Clin. Nutr. 84, 1481-1488 (2006).

380. Pereira, M. A. et al. Dairy Consumption, Obesity, and the Insulin Resistance Syndrome in Young Adults: The CARDIA Study. JAMA 287, 2081-2089 (2002).

381. Wang, H. et al. Obesity Modifies the Relations Between Serum Markers 
of Dairy Fats and Inflammation and Oxidative Stress Among Adolescents. Obesity 19, 2404-2410 (2011).

382. Cecil, J. E., Francis, J. \& Read, N. W. Comparison of the effects of a high-fat and high-carbohydrate soup delivered orally and intragastrically on gastric emptying, appetite, and eating behaviour. Physiol. Behav. 67, 299-306 (1999).

383. FSA Nutrient and Food Based Guidelines for UK Institutions. Food Standards Agency Available at: http://multimedia.food.gov.uk/multimedia/pdfs/nutguideuk.pdf. (Accessed: 13th March 2014)

384. Siri-Tarino, P. W., Sun, Q., Hu, F. B. \& Krauss, R. M. Saturated fatty acids and risk of coronary heart disease: Modulation by replacement nutrients. Curr. Atheroscler. Rep. 12, 384-390 (2010).

385. Briggs, M., Petersen, K. \& Kris-Etherton, P. Saturated Fatty Acids and Cardiovascular Disease: Replacements for Saturated Fat to Reduce Cardiovascular Risk. Healthcare 5, 29 (2017).

386. Siri-tarino, P. W., Sun, Q., Hu, F. B. \& Krauss, R. M. Meta-analysis of prospective cohort studies evaluating the association of saturated fat with cardiovascular disease. Am. J. Clin. Nutr. 91, 535-546 (2010).

387. Huth, P. J. \& Park, K. M. Influence of Dairy Product and Milk Fat Consumption on Cardiovascular Disease Risk : A Review of the Evidence. Adv. Nutr. 3, 266-285 (2012).

388. Staiano, A. E., Gupta, A. K. \& Katzmarzyk, P. T. Cardiometabolic risk factors and fat distribution in children and adolescents. J. Pediatr. 164, 560-5 (2014).

389. Teixeira, P. J., Sardinha, L. B., Going, S. B. \& Lohman, T. G. Total and regional fat and serum cardiovascular disease risk factors in lean and obese children and adolescents. Obes. Res. 9, 432-42 (2001).

390. Ford, A. L., Hunt, L. P., Cooper, A. \& Shield, J. P. H. What reduction in BMI SDS is required in obese adolescents to improve body composition and cardiometabolic health? Arch. Dis. Child. 95, 256-261 (2010).

391. Kolsgaard, M. L. P., Joner, G., Brunborg, C., Anderssen, S. A. \& Tonstad, $\mathrm{S}$. Reduction in BMI z-score and improvement in cardiometabolic risk factors in obese children and adolescents . The Oslo Adiposity Intervention Study - a hospital / public health nurse combined treatment. BMC Pediatr. 11, 47 (2011).

392. van de Mortel, T. Faking it : social desirability response bias in self- report research. Aust. J. Adv. Nurs. 25, 40-48 (2008).

393. Baquet, G., Praagh, E. Van \& Berthoin, S. Endurance Training and Aerobic Fitness in Young People. 33, 1127-1143 (2003).

394. Ortega, F. B., Ruiz, J. R., Hurtig-wennlöf, A. \& Sjöström, M. Physically Active Adolescents Are More Likely to Have a Healthier Cardiovascular Fitness Level Independently of Their Adiposity Status . The European Youth Heart Study. 123-129 (2008).

395. Boddy, L. M. et al. ROC generated thresholds for field-assessed aerobic fitness related to body size and cardiometabolic risk in schoolchildren. PLoS One 7, e45755 (2012).

396. Hubert HB, Feinleib M, McNamara PM, C. W. Obesity as an independent risk factor for cardiovascular disease: a 26-year follow-up of participants in the Framingham Heart Study. Circulation 67, 968-77 (1983). 
397. Abbasi, A., Juszczyk, D., van Jaarsveld, C. H. \& Gulliford, M. C. Body Mass Index and Incident Type 1 and Type 2 Diabetes in Children and Young Adults: A Retrospective Cohort Study. J. Endocr. Soc. 1, 524-537 (2017).

398. Li, G. et al. Cardiovascular mortality, all-cause mortality, and diabetes incidence after lifestyle intervention for people with impaired glucose tolerance in the Da Qing Diabetes Prevention Study: A 23-year follow-up study. Lancet Diabetes Endocrinol. 2, 474-480 (2014).

399. Smith, K. L., Straker, L. M., McManus, A. \& Fenner, A. Barriers and enablers for participation in healthy lifestyle programs by adolescents who are overweight: a qualitative study of the opinions of adolescents, their parents and community stakeholders. BMC Pediatr. 14, 53 (2014).

400. Morrison, Z., Gregory, D., Thibodeau, S. \& Copeland, J. Ouch! Recruitment of overweight and obese adolescent boys for qualitative research. Qual. Rep. 17, 1-17 (2012).

401. Nguyen, B. et al. Recruitment challenges and recommendations for adolescent obesity trials. J. Paediatr. Child Health 48, 38-43 (2012).

402. Tyler, R., Mackintosh, K., Palmer, A., Jones, A. \& Stratton, G. Ten-Year Secular Changes in Selected Health and Fitness Parameters of 10-11 Years Old Swansea School Children. Adv. Obesity, Weight Manag. Control 3, 8-13 (2015).

403. Kurtoğlu, S. et al. Insulin resistance in obese children and adolescents: HOMA-IR cut-off levels in the prepubertal and pubertal periods. J. Clin. Res. Pediatr. Endocrinol. 2, 100-106 (2010).

404. Schalkwijk, A. et al. Perspectives of obese children and their parents on lifestyle behavior change: A qualitative study. Int. J. Behav. Nutr. Phys. Act. 12, 1-8 (2015).

405. Harden, S. M., Ramalingam, N. S., Wilson, K. E. \& Evans-Hoeker, E. Informing the development and uptake of a weight management intervention for preconception: a mixed-methods investigation of patient and provider perceptions. BMC Obes. 4, 8 (2017).

406. ADA. Diagnosis and classification of diabetes mellitus. Diabetes Care 33, (2010).

407. van Sluijs, E. M. F., McMinn, A. M. \& Griffin, S. J. Effectiveness of interventions to promote physical activity in children and adolescents: systematic review of controlled trials. Bmj 335, 703-703 (2007).

408. Tucker, P. \& Gilliland, J. The effect of season and weather on physical activity: a systematic review. Public Health 121, 909-22 (2007).

409. Prochaska, J. O., DiClemente, C. C. \& Norcross, J. C. In search of how people change. Applications to addictive behaviors. Am. Psychol. 47, 1102-14 (1992).

410. Tannenbaum, M. B. et al. Appealing to Fear: A Meta-Analysis of Fear Appeal Effectiveness and Theories. Am. Psychol. Assoc. 141, 1178-1204 (2015).

411. Witte, K. \& Allen, M. A meta-analysis of fear appeals: implications for effective public health campaigns. Health Educ. Behav. 27, 591-615 (2000).

412. Kwasnicka, D., Dombrowski, S. U., White, M. \& Sniehotta, F. Theoretical explanations for maintenance of behaviour change: a systematic review of behaviour theories. Health Psychol. Rev. 10, 277-296 (2016). 
413. Baker, R. C. \& Kirschenbaum, D. S. Self-monitoring may be necessary for successful weight control. Behav. Ther. 24, 377-394 (1993).

414. Germann, J. N., Kirschenbaum, D. S. \& Rich, B. H. Child and Parental Self-Monitoring as Determinants of Success in the Treatment of Morbid Obesity in Low-Income Minority Children. J. Pediatr. Psychol. 32, 111121 (2007).

415. Schwarzer, R. \& Renner, B. Social-cognitive predictors of health behavior: Action self-efficacy and coping self-efficacy. Heal. Psychol. 19, 487-495 (2000).

416. Markland, D., Ryan, R., Tobin, V. \& Rollnick, S. Motivational Interviewing and Self-Determination Theory. J. Soc. Clin. Psychol. 24, 811-831 (2005).

417. Herzer, M., Zeller, M. H., Rausch, J. R. \& Modi, A. C. Perceived Social Support and Its Association With Obesity-Specific Health-related Quality of Life. J Dev Behav Pediatr 32, 188-195 (2012).

418. Ventura, A. K. \& Birch, L. L. Does parenting affect children's eating and weight status? Int. J. Behav. Nutr. Phys. Act. 5, (2008).

419. Salvy, S.-J., Keiffer, E. \& Epstein, L. H. Effects of social context on overweight and normal-weight children's food selection. Eat. Behav. 9, 190-196 (2008).

420. Michie, S. et al. A refined taxonomy of behaviour change techniques to help people change their physical activity and healthy eating behaviours: The CALO-RE taxonomy. Psychol. Heal. 26, 1479-1498 (2011).

421. NHS England » NHS Diabetes Prevention Programme National Service Specification. Available at: https://www.england.nhs.uk/publication/nhsdpp-national-service-specification/. (Accessed: 13th June 2018)

422. Department of Health Physical Activity Health Improvement and Protection. Start Active, Stay Active. Report 62 (2011). doi:https://www.gov.uk/government/uploads/system/uploads/attachment_ data/file/216370/dh_128210.pdf

423. Department of Health and Social Care (Institution or Organization). NHS Diabetes Prevention Programme: An opportunity to partner with the Behavioural Insight Team to improve outcomes. Available at: https://www.england.nhs.uk/wp-content/uploads/2016/07/behavinsight.pdf. (Accessed: 13th June 2018)

424. Chen, M., Pan, A., Malik, V. \& Hu, F. Effects of dairy intake on body weight and fat: a meta-analysis of randomized controlled trials. Am. J. Clin. Nutr. 96, 735-747 (2012).

425. Chastin, S. F. M., Palarea-Albaladejo, J., Dontje, M. L. \& Skelton, D. A. Combined effects of time spent in physical activity, sedentary behaviors and sleep on obesity and cardio-metabolic health markers: A novel compositional data analysis approach. PLoS One 10, (2015).

426. Rhodes, R. \& Pfaeffli, L. Mediators of physical activity behaviour change among adult non-clinical populations: a review update. Int. J. Behav. Nutr. Phys. Act. 7, 11p-11p (2010). 


\section{Appendices}

\subsection{Appendix 1 Missing data for study 1}

\begin{tabular}{|c|c|c|c|}
\hline Variables & $\mathrm{N}$ & Missing Count & Percent \\
\hline Age & 2223 & 103 & 4.4 \\
\hline Sex & 2199 & 127 & 5.4 \\
\hline BMI & 2314 & 12 & 0.5 \\
\hline Site & 2326 & 0 & 0 \\
\hline Ethnicity & 2326 & 0 & 0 \\
\hline Smoking & 2321 & 5 & 0.2 \\
\hline Household income & 2076 & 250 & 10.7 \\
\hline Education & 2149 & 177 & 7.6 \\
\hline Body fat $\%$ & 2160 & 166 & 7.1 \\
\hline Waist circumference & 2193 & 133 & 5.7 \\
\hline Diastolic BP & 2314 & 12 & 0.5 \\
\hline Systolic BP & 2322 & 4 & 0.2 \\
\hline Fasting plasma glucose & 2318 & 8 & 0.3 \\
\hline Fasting insulin & 2298 & 28 & 1.2 \\
\hline HOMA_IR & 2293 & 33 & 1.4 \\
\hline $2 \mathrm{~h}$ plasma glucose & 2325 & 1 & 0 \\
\hline Total cholesterol & 2325 & 1 & 0 \\
\hline HDL-cholesterol & 2325 & 1 & 0 \\
\hline LDL-cholesterol & 2325 & 1 & 0 \\
\hline Triglycerides & 2194 & 132 & 5.6 \\
\hline Hba1C & 2320 & 6 & 0.2 \\
\hline Hba1C \% & 2317 & 9 & 0.3 \\
\hline CRP & 2223 & 103 & 4.4 \\
\hline $\begin{array}{l}\text { Accelerometer variables } \\
\text { Waking wear Time } \\
(\text { minutes } \cdot \text { day } \\
\text { Sleep }\left(\text { minutes } \cdot d a y^{-1}\right)\end{array}$ & 2309 & $\begin{array}{l}17 \\
612\end{array}$ & 0.7 \\
\hline Sedentary (minutes $\cdot d a y^{-1}$ ) & $1 / 14$ & 612 & 26.3 \\
\hline Light (minutes $\left.\cdot d a y^{-1}\right)$ & 1923 & 403 & 17.3 \\
\hline Moderate (minutes $\cdot$ day ${ }^{-1}$ ) & 1923 & 403 & 17.3 \\
\hline MVPA $\left(\right.$ minutes $\left.\cdot d a y^{-1}\right)$ & 1923 & 403 & 17.3 \\
\hline$C P M\left(\right.$ counts $^{\prime} \cdot$ minute $\left.^{-1}\right)$ & 1923 & 403 & 17.3 \\
\hline
\end{tabular}

BP, Blood Pressure, $\mathrm{HbA}_{1 \mathrm{c}}$, Glycated haemoglobin A1c, HOMA-IR, Homeostatic Model Assessment Insulin Resistance, HDL, High-Density

Lipoprotein, LDL, Low-Density Lipoprotein, CRP, C-reactive protein. 


\subsection{Appendix 2 Missing data study 2}

\begin{tabular}{|c|c|c|c|}
\hline & $\mathrm{N}$ & Missing Count & Percent \\
\hline Age & 2223 & 104 & 4.5 \\
\hline Sex & 2326 & 0 & 0 \\
\hline Body fat $\%$ & 2160 & 166 & 7.1 \\
\hline Ethnicity & 2319 & 7 & 0.3 \\
\hline Smoking & 2197 & 129 & 5.5 \\
\hline Day light & 2223 & 103 & 4.4 \\
\hline MVPA & 1922 & 403 & 17.3 \\
\hline Time for PA & 2198 & 128 & 5.5 \\
\hline Place for PA & 2169 & 157 & 6.7 \\
\hline Social support friends & 2025 & 301 & 12.9 \\
\hline Social support family & 2070 & 256 & 11 \\
\hline Friends discourage & 2164 & 162 & 7 \\
\hline Family discourage & 2164 & 162 & 7 \\
\hline Inactivity temptations & 2197 & 129 & 5.5 \\
\hline Goal disengagement & 2153 & 173 & 7.4 \\
\hline Goal reengagement & 2117 & 209 & 9 \\
\hline Self-efficacy & 2152 & 174 & 7.5 \\
\hline Habit strength sitting & 2158 & 168 & 7.2 \\
\hline Habit strength transport & 2121 & 205 & 8.8 \\
\hline Intentions to exercise & 2141 & 185 & 8 \\
\hline Internal/External) & 2000 & 326 & 14.1 \\
\hline Stable/unstable stable & 1678 & 648 & 27.8 \\
\hline Control Others & 1734 & 592 & 25.4 \\
\hline Control Self & 1827 & 499 & 21.4 \\
\hline Education & 2150 & 176 & 7.6 \\
\hline SES & 2076 & 250 & 10.7 \\
\hline
\end{tabular}

MVPA, moderate-to-vigorous physical activity, PA, physical activity, SES socio-economic status 


\subsection{Appendix 3 Missing data study 3}

\begin{tabular}{|c|c|c|c|}
\hline Variables & $\mathrm{N}$ & Missing Count & Percent \\
\hline Age & 12672 & 3305 & 20.7 \\
\hline IMD Score & 14871 & 1106 & 6.9 \\
\hline Body fat $\%$ & 14387 & 1590 & 10 \\
\hline Eat breakfast? & 13375 & 2602 & 16.3 \\
\hline Drink Breakfast? & 13302 & 2675 & 16.7 \\
\hline Eat on the way to school? & 13171 & 2806 & 17.6 \\
\hline Drink on the way to school? & 13099 & 2878 & 18 \\
\hline School lunch? & 13101 & 2876 & 18 \\
\hline Packed lunch? & 13040 & 2937 & 18.4 \\
\hline Home for lunch? & 12724 & 3253 & 20.4 \\
\hline Takeaway foods? & 13293 & 2684 & 16.8 \\
\hline Week TV $\geq 2 \mathrm{hs}$ & 12918 & 3059 & 19.1 \\
\hline Week Internet $\geq 2 \mathrm{hs}$ & 12716 & 3261 & 20.4 \\
\hline Week Computer $\geq 2 \mathrm{hs}$ & 12814 & 3163 & 19.8 \\
\hline Week Homework $\geq 2 \mathrm{hs}$ & 12589 & 3388 & 21.2 \\
\hline Week Sport $\geq 2 \mathrm{hs}$ & 12807 & 3170 & 19.8 \\
\hline Weekend TV $\geq 2 \mathrm{hs}$ & 12699 & 3278 & 20.5 \\
\hline Weekend Internet $\geq 2 \mathrm{hs}$ & 12544 & 3433 & 21.5 \\
\hline Weekend Computer $\geq 2 \mathrm{hs}$ & 12598 & 3379 & 21.1 \\
\hline Weekend Homework $\geq 2 \mathrm{hs}$ & 12372 & 3605 & 22.6 \\
\hline Weekend Sport $\geq 2 \mathrm{hs}$ & 12626 & 3351 & 21 \\
\hline Active transport to school & 13050 & 2927 & 18.3 \\
\hline Bed time & 11857 & 4120 & 25.8 \\
\hline Sugar sweetened cereals & 13113 & 2864 & 17.9 \\
\hline High fibre cereals & 13095 & 2882 & 18 \\
\hline Oat based cereals & 12969 & 3008 & 18.8 \\
\hline $\begin{array}{l}\text { Other non-sugar sweetened } \\
\text { cereals }\end{array}$ & 12906 & 3071 & 19.2 \\
\hline White bread & 13078 & 2899 & 18.1 \\
\hline Brown bread & 12575 & 3402 & 21.3 \\
\hline Butter & 13220 & 2757 & 17.3 \\
\hline Hard margarine & 12949 & 3028 & 19 \\
\hline Soft margarine & 12932 & 3045 & 19.1 \\
\hline Polly unsaturated margarine & 12938 & 3039 & 19 \\
\hline Low fat margarine & 12497 & 3480 & 21.8 \\
\hline Plain biscuits & 13050 & 2927 & 18.3 \\
\hline Chocolate biscuits & 12997 & 2980 & 18.7 \\
\hline Cakes & 13317 & 2660 & 16.6 \\
\hline Puddings & 13168 & 2809 & 17.6 \\
\hline Sweets & 13203 & 2774 & 17.4 \\
\hline
\end{tabular}




\begin{tabular}{llll}
\hline Chocolate bars & 13159 & 2818 & 17.6 \\
Ice cream & 12986 & 2991 & 18.7 \\
Sugar in drinks & 13208 & 2769 & 17.3 \\
Sugar on food & 13189 & 2788 & 17.5 \\
Boiled potatoes & 12914 & 3063 & 19.2 \\
Mashed potatoes & 12985 & 2992 & 18.7 \\
Jacket potatoes & 12932 & 3045 & 19.1 \\
Roast potatoes & 12934 & 3043 & 19 \\
Chips & 13047 & 2930 & 18.3 \\
Crisps & 13067 & 2910 & 18.2 \\
Fresh fruit & 13242 & 2735 & 17.1 \\
Baked beans & 13018 & 2959 & 18.5 \\
Fried veg & 13142 & 2835 & 17.7 \\
Salad & 12957 & 3020 & 18.9 \\
Other Veg & 13161 & 2816 & 17.6 \\
Burgers ordinary & 13085 & 2892 & 18.1 \\
Sausages ordinary & 13084 & 2893 & 18.1 \\
Burgers low fat & 13025 & 2952 & 18.5 \\
Sausages low fat & 13022 & 2955 & 18.5 \\
Meat pies & 13001 & 2976 & 18.6 \\
Other meat & 13135 & 2842 & 17.8 \\
Fried fish & 13071 & 2906 & 18.2 \\
Other fish & 13073 & 2904 & 18.2 \\
Ordinary cheese & 13229 & 2748 & 17.2 \\
Soft cheese & 13152 & 2825 & 17.7 \\
Low fat cheese & 12841 & 3136 & 19.6 \\
Fizzy drinks & 13165 & 2812 & 17.6 \\
Diet fizzy drinks & 12972 & 3005 & 18.8 \\
Sugar sweetened beverages & 13105 & 2872 & 21.2 \\
Low calorie beverages & 12596 & 3381 & 3335 \\
Full fat milk & 12642 & 2972 & \\
Semi skimmed or skimmed & 13005 & & \\
milk & & 38.6 \\
\hline
\end{tabular}




\subsection{Appendix 4 Physical activity hand book}
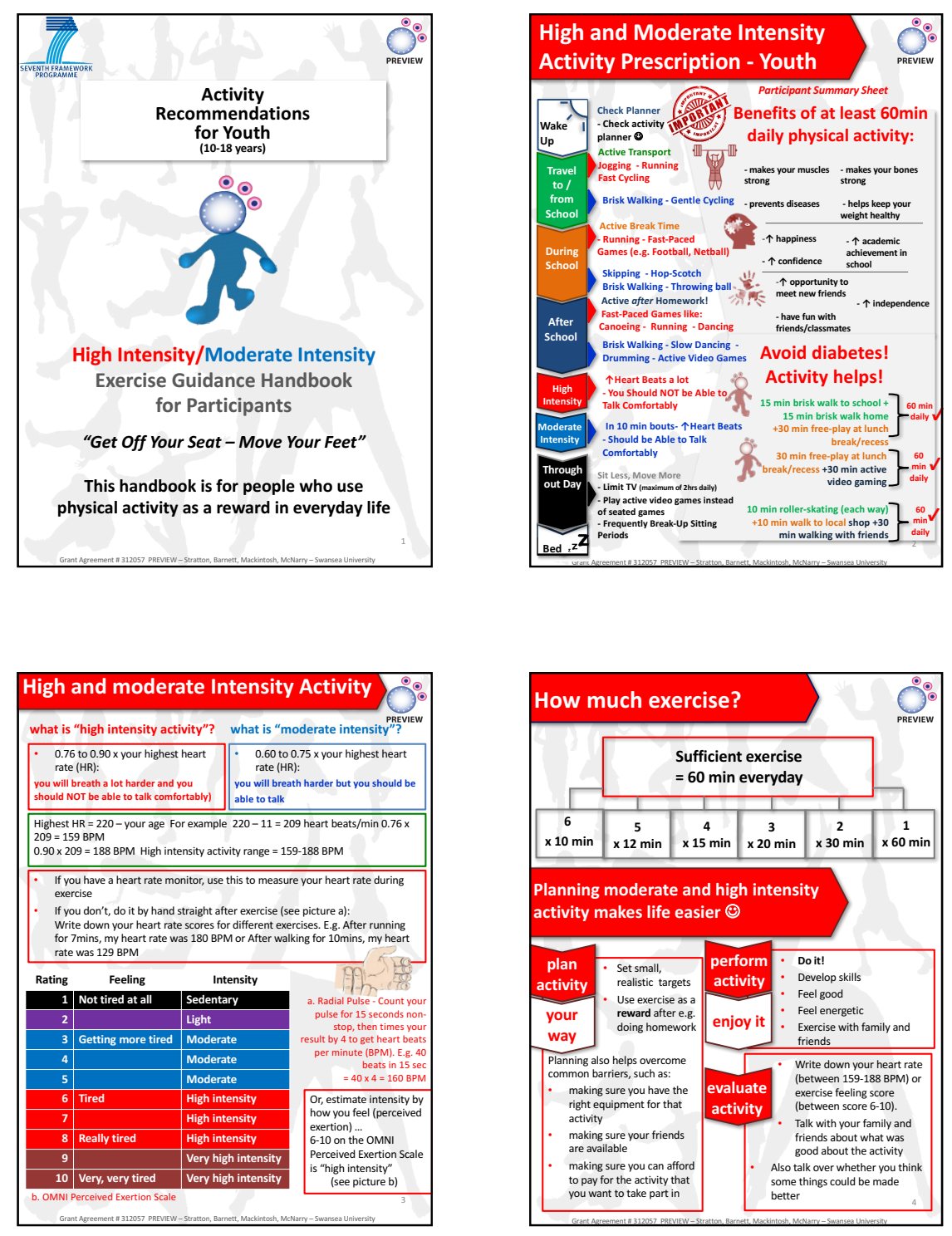

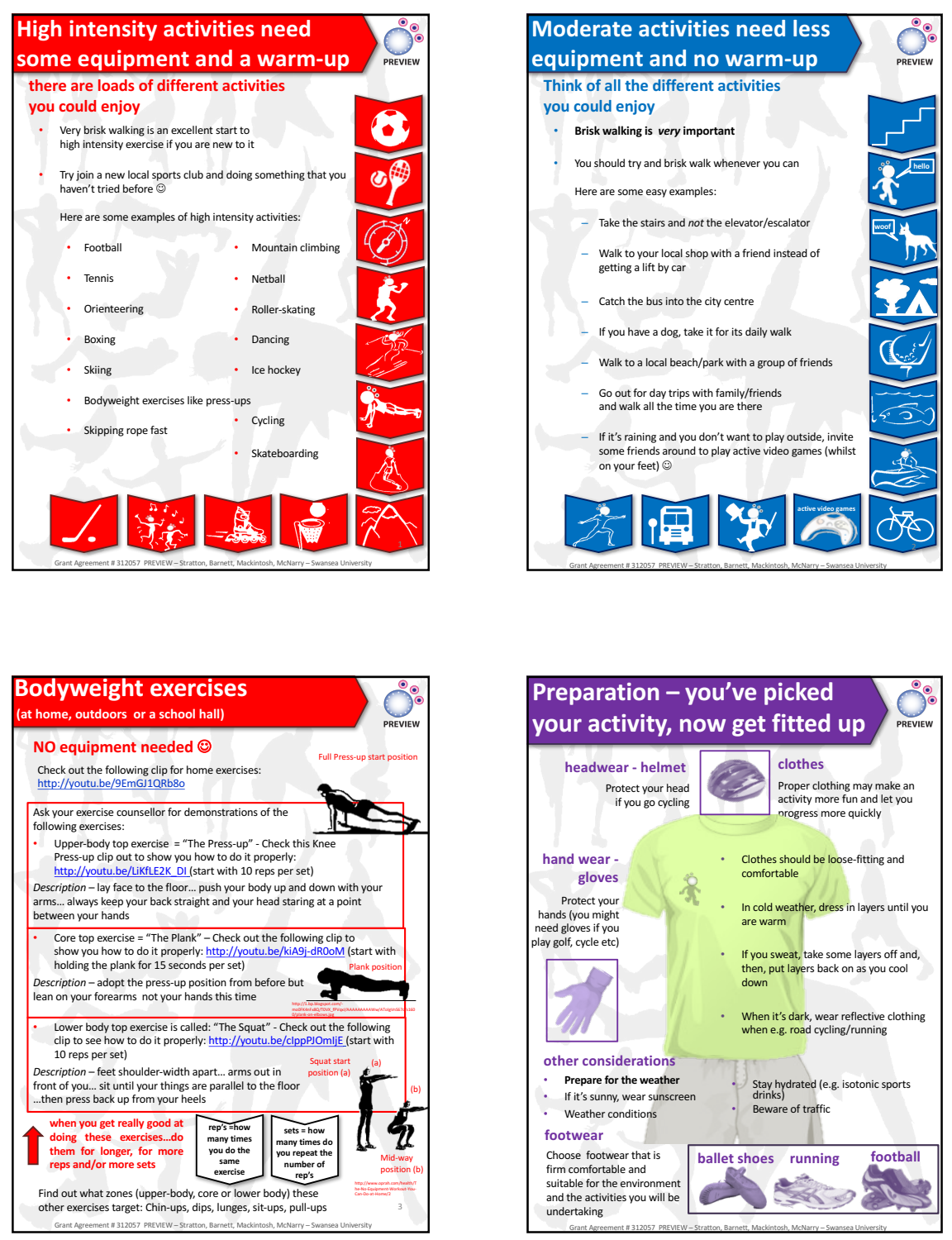

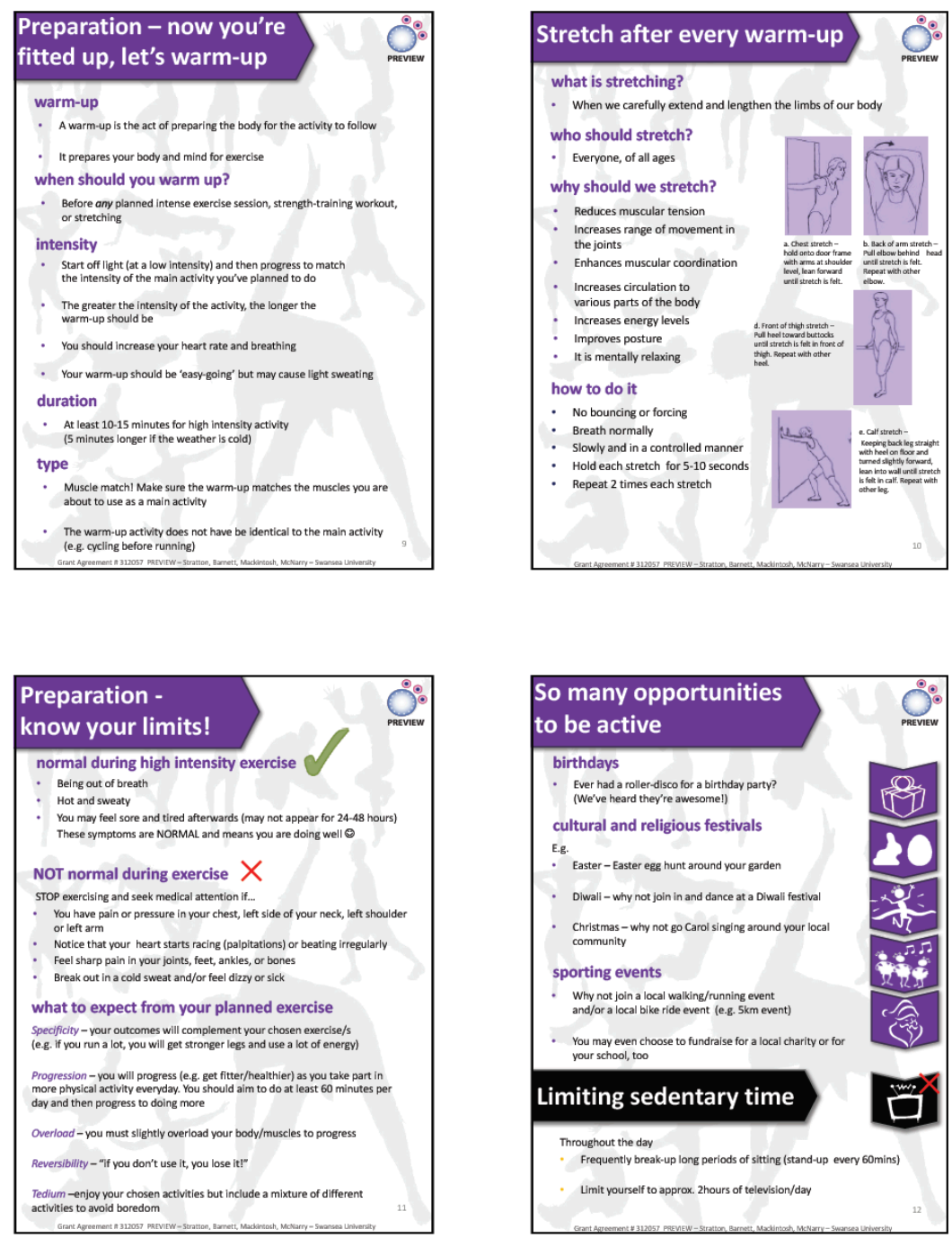

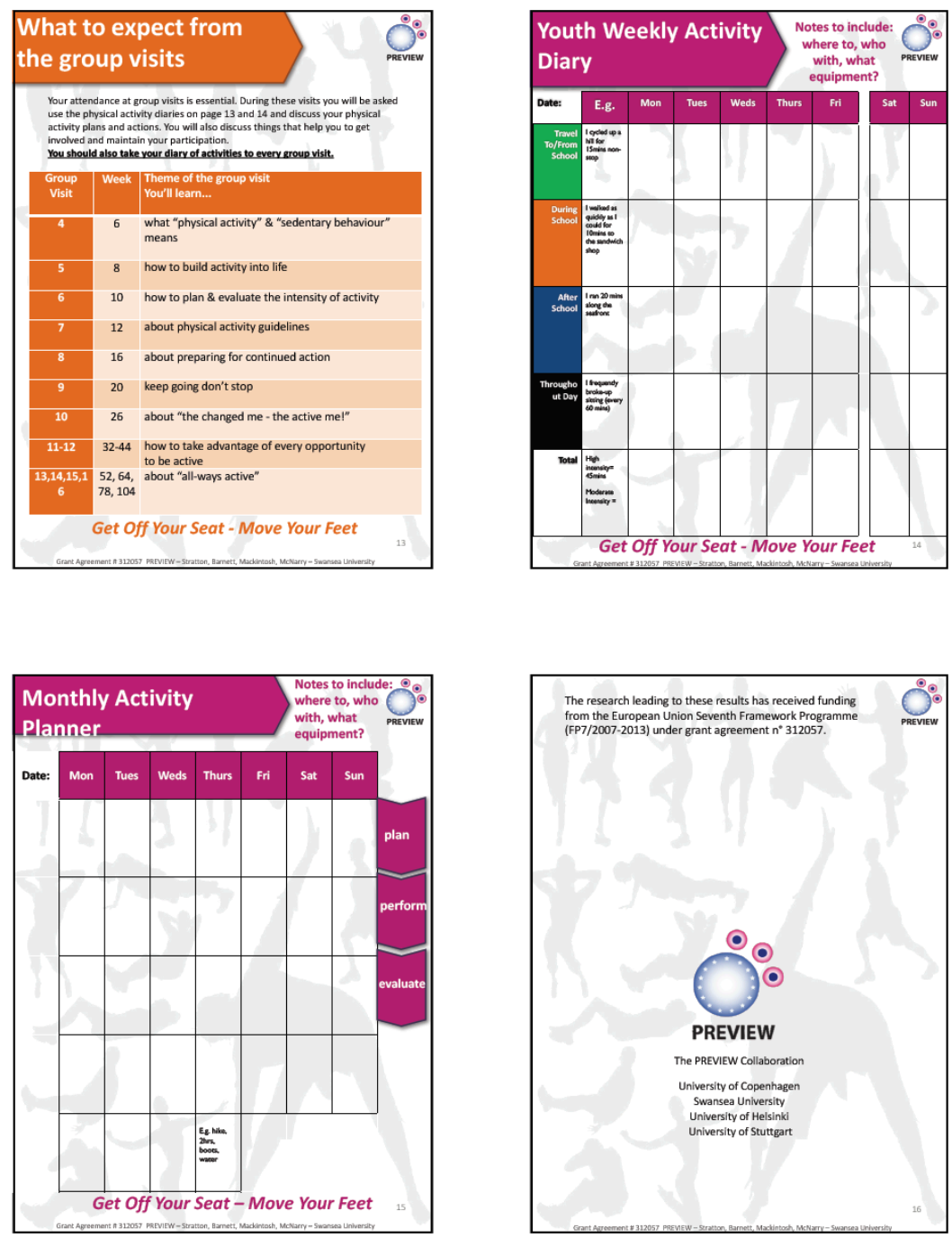


\subsection{Appendix 5 Behaviour Change Toolbox}

PREVIEW Behavior Modification Intervention Toolbox (PREMIT): A Study Protocol for a Psychological Ele Kahlert et al $2016^{296}$

\begin{tabular}{|c|c|c|c|c|c|}
\hline $\begin{array}{c}\text { Intervention } \\
\text { Phases } \\
\end{array}$ & Objectives & $\begin{array}{l}\text { Group } \\
\text { Visit }\end{array}$ & Technique(s) & Tool(s) & $\begin{array}{c}\text { References } \\
\text { (selected) }\end{array}$ \\
\hline \multirow{4}{*}{$\begin{array}{l}\text { week 1-8 } \\
\text { Weight } \\
\text { reduction } \\
\text { phase } \\
\text { (LED) } \\
\text { GV } 1 \text { - GV } 5\end{array}$} & $\begin{array}{l}\text { - enable your clients to } \\
\text { follow LCD }\end{array}$ & 1 & $\begin{array}{l}\text { - provide information about } \\
\text { the LCD period and instruct } \\
\text { clients }\end{array}$ & Wirtten material & \\
\hline & $\begin{array}{l}\text { - convince clients to } \\
\text { change behaviour }\end{array}$ & 2,3 & -fear appeal/arousal (32) & $\begin{array}{l}\text {-persuasion (central } \\
\text { route) }\end{array}$ & $\begin{array}{l}\text {-Witte \& Allen } \\
(2000)\end{array}$ \\
\hline & $\begin{array}{l}\text { promote actional self- } \\
\text { efficacy }\end{array}$ & $2,3,4$ & $\begin{array}{l}\text { •prompting past success (18) } \\
\text { •modelling/demonstrate (22) }\end{array}$ & $\begin{array}{l}\text {-persuasion (central } \\
\text { route) }\end{array}$ & $\begin{array}{l}\text {-Willams et. al. } \\
\text { (2011) } \\
\text {-Olander et a. } \\
(2013)\end{array}$ \\
\hline & $\begin{array}{l}\text { - convince clients that the } \\
\text { recommended behaviour } \\
\text { will be followed by } \\
\text { positive outcomes }\end{array}$ & 3,4 & $\begin{array}{l}\text {-information on individual } \\
\text { consequences (2) }\end{array}$ & $\begin{array}{l}\text {-persuasion (central } \\
\text { route) }\end{array}$ & $\begin{array}{l}\text { Petty \& Cacioppo } \\
(1986)\end{array}$ \\
\hline \multirow{6}{*}{$\begin{array}{c}\text { week 8-12 (p.a. } \\
16 ?) \\
\text { Preparation } \\
\text { GV } 5 \text { - GV } 7 \\
\text { Overall } \\
\text { objective: } \\
\text { Enable clients } \\
\text { to begin, } \\
\text { follow and } \\
\text { train the "new" } \\
\text { behaviour }\end{array}$} & $\begin{array}{l}\text { - help defining SMART } \\
\text { goals }\end{array}$ & 5 & -goal setting (5) & $\begin{array}{l}\text {-template for dfining } \\
\text { SMART goals }\end{array}$ & $\begin{array}{l}\cdot \text {-Greaves et al. } \\
(2011) ; \cdot \text { Locke \& } \\
\text { Latham (1990); } \\
\text { •Gollwitzer (1993) }\end{array}$ \\
\hline & - help defining plans & 5 & -action planning (7) (20) & $\begin{array}{l}\text { - template for action } \\
\text { planning }\end{array}$ & -Gollwitzer (1993) \\
\hline & - help to commit to goals & 5 & - contracting (25) & $\begin{array}{l}\text { - template for } \\
\text { contracting }\end{array}$ & $\begin{array}{l}\text {-(Conn et. al., } \\
2008) ; ?\end{array}$ \\
\hline & - promote self-efficacy & 6 & -comparison to others & $\begin{array}{l}\text { provide information } \\
\text { a) approval of others } \\
\text { b) behaviour of others } \\
(3,4)\end{array}$ & $\begin{array}{l}\text {-Willams et. al. } \\
(2011) \\
\text {-Olander et a. } \\
(2013)\end{array}$ \\
\hline & $\begin{array}{l}\text { - foster positive outcome } \\
\text { expectancies }\end{array}$ & 6 & $\begin{array}{l}\text {-information on individual } \\
\text { consequences (2) }\end{array}$ & $\begin{array}{l}\text {-persuasion (central } \\
\text { route) }\end{array}$ & $\begin{array}{l}\cdot(\text { Conn et. al., } \\
2008)\end{array}$ \\
\hline & $\begin{array}{l}\text { - enable clients to } \\
\text { overcome barriers }\end{array}$ & 7 & $\begin{array}{l}\text {-barrier identification/problem } \\
\text { solving (8) }\end{array}$ & $\begin{array}{l}\text {-mental contrasting } \\
\text { technique }\end{array}$ & $\begin{array}{l}\text { - Adrianse et al. } \\
(2010) \\
\text { Oettingen \& } \\
\text { Gollwitzer (2010) }\end{array}$ \\
\hline
\end{tabular}




\begin{tabular}{|c|c|c|c|c|c|}
\hline $\begin{array}{l}\text { Intervention } \\
\text { Phases }\end{array}$ & Objectives & $\begin{array}{l}\text { Group } \\
\text { Visit }\end{array}$ & Technique(s) & Tool(s) & $\begin{array}{l}\text { References } \\
\text { (selected) }\end{array}$ \\
\hline \multirow{6}{*}{$\begin{array}{l}\text { week 8-12 (p.a. } \\
\text { 16?) } \\
\text { Preparation } \\
\text { GV } 5 \text { - GV } 7 \\
\text { Overall } \\
\text { objective: } \\
\text { Enable clients } \\
\text { to begin, follow } \\
\text { and train the } \\
\text { "new" } \\
\text { behaviour }\end{array}$} & $\begin{array}{l}\text { - promote/ support self- } \\
\text { regulation }\end{array}$ & 6 & $\begin{array}{l}\text {-prompt review of behavioural } \\
\text { goals (10) (16) (19), prompt } \\
\text { self-monitoring of behav }\end{array}$ & $\begin{array}{l}\text {-PA log respective } \\
\text { food diary }\end{array}$ & $\begin{array}{l}\cdot \text { Carver \& Scheier } \\
\text { (1998) } \\
\text { •Conn et al. (1998) }\end{array}$ \\
\hline & $\begin{array}{l}\text { - help clients find social } \\
\text { support }\end{array}$ & 7 & •plan social support (29) & •buddy-system & $\begin{array}{l}\text {-Greaves et al. } \\
\text { (2011) }\end{array}$ \\
\hline & \multirow{4}{*}{$\begin{array}{l}\text { - enable sujects to stick } \\
\text { to the new behaviour }\end{array}$} & 6,7 & $\begin{array}{l}\text {-prompt rewards contingent } \\
\text { (12) } \quad \text { provide rewards } \\
\text { (13) }\end{array}$ & $\begin{array}{l}\text {-Shaping (Phase 1) } \\
\text { (14) }\end{array}$ & $\begin{array}{l}\cdot \text { Deci \& Ryan } \\
(2000) \quad \text { Mazur } \\
\text { Lernen und } \\
\text { Verhalten? }\end{array}$ \\
\hline & & 7 & $\begin{array}{l}\text {-prompt focus on past success } \\
\text { (18) }\end{array}$ & & \\
\hline & & 6,7 & $\begin{array}{l}\text {-provide feedback on } \\
\text { performance (19) }\end{array}$ & & $\begin{array}{l}\text { - Greaves et al. } \\
(2011)\end{array}$ \\
\hline & & 5 & $\begin{array}{l}\text {-provide information on how } \\
\text { to perform the behaviour }(21)\end{array}$ & & \\
\hline \multirow{5}{*}{$\begin{array}{c}\text { week } 16-26 \\
\text { (recommended } \\
\text { p.a. } 16 ?-26 \text { ) } \\
\text { Action } \\
\text { GV } 8 \text { - GV } 10 \\
\text { Overall } \\
\text { objective: } \\
\text { Enable clients } \\
\text { to stick to the } \\
\text { recommended } \\
\text { behaviour }\end{array}$} & - promote self-efficacy & 8 & $\begin{array}{l}\text {-prompt focus on past success } \\
(18) ; \cdot \text { provide Feedback on } \\
\text { performance }(19) ; \cdot \text { provide } \\
\text { information on how to } \\
\text { perform the behaviour }(21)\end{array}$ & & $\begin{array}{l}\text {-Willams et. al. } \\
\text { (2011) } \\
\text {-Olander et a. } \\
(2013)\end{array}$ \\
\hline & \multirow{2}{*}{$\begin{array}{l}\text { - enable clients to } \\
\text { overcome barriers }\end{array}$} & 8 & $\begin{array}{l}\text {-barrier identification/problem } \\
\text { solving ( } 8 \text { ) }\end{array}$ & •mental contrasting & $\begin{array}{l}\text {-Adrianse et al. } \\
(2010)\end{array}$ \\
\hline & & 8 & $\begin{array}{l}\text {-environmental restructuring } \\
(24)\end{array}$ & $\begin{array}{l}\text {-stimulus control; } \\
\text { decision-prompts }\end{array}$ & \\
\hline & \multirow{2}{*}{$\begin{array}{l}\text { - promote/ support self- } \\
\text { regulation }\end{array}$} & \multirow[t]{2}{*}{9} & $\begin{array}{l}\text {-prompt review of behavioural } \\
\text { goals (10) (16) (19) }\end{array}$ & $\begin{array}{l}\text { - pa log respective } \\
\text { food diary }\end{array}$ & $\begin{array}{l}\text {-Carver \& Scheier } \\
\text { (1998) }\end{array}$ \\
\hline & & & -prompt self-talk (33) & & $\begin{array}{l}\cdot \text { Greaves et al. } \\
(2011) ; \cdot \text { Neck \& } \\
\text { Manz }(1992) ; \\
\text { Meichenbaum } \\
(1987)\end{array}$ \\
\hline
\end{tabular}




\section{Appendix 5 continued}

\begin{tabular}{|c|c|c|c|c|c|}
\hline $\begin{array}{c}\text { Intervention } \\
\text { Phases } \\
\end{array}$ & Objectives & $\begin{array}{l}\text { Group } \\
\text { Visit } \\
\end{array}$ & Technique(s) & Tool(s) & $\begin{array}{l}\text { References } \\
\text { (selected) }\end{array}$ \\
\hline & & 10 & -prompt use of imagery (34) & -mental contrasting & \\
\hline & & 10 & $\begin{array}{l}\text {-prompt self-monitoring of } \\
\text { behaviour }(16)\end{array}$ & $\begin{array}{l}\text {-pa log respective } \\
\text { food diary }\end{array}$ & $\begin{array}{l}\text {-Greaves et al. } \\
(2011)\end{array}$ \\
\hline & & 10 & $\begin{array}{l}\text {-provide feedback on } \\
\text { performance }(19)\end{array}$ & & $\begin{array}{l}\text {-Greaves et al. } \\
(2011)\end{array}$ \\
\hline & & 8 & $\begin{array}{l}\text {-teach to use prompts/ cues } \\
\text { (23) }\end{array}$ & •decision-prompts & -Conn et al. (2008) \\
\hline & & 10 & $\begin{array}{l}\text {-provide of follow-up prompts } \\
\text { (27) }\end{array}$ & •decision-prompts & \\
\hline & $\begin{array}{l}\text { - enhance self-regulation } \\
\text { of motivation }\end{array}$ & 9,10 & $\begin{array}{l}(\bullet \text { prompt rewards contingent } \\
(12)) \quad \text { •provide } \\
\text { rewards (13) }\end{array}$ & $\begin{array}{l}\text { •shaping (Phase 2) } \\
\text { (14) }\end{array}$ & $\begin{array}{l}\text {-Deci \& Ryan } \\
(2000)\end{array}$ \\
\hline & $\begin{array}{l}\text { - help clients finding } \\
\text { social support }\end{array}$ & 9 & •plan social support (29) & •buddy-system & $\begin{array}{l}\text {-Greaves et al. } \\
(2011)\end{array}$ \\
\hline \multirow{5}{*}{$\begin{array}{c}\text { week } 26-156 \\
\text { Adherence } \\
\text { GV } 11-\mathrm{GV} 18 \\
\text { Overall } \\
\text { objective: } \\
\text { Enable clients } \\
\text { to prevent } \\
\text { lapses and } \\
\text { relapses }\end{array}$} & \multirow{2}{*}{$\begin{array}{l}\text { - enhance coping abilities } \\
\text { and coping self- } \\
\text { regulation }\end{array}$} & \multirow[t]{2}{*}{11,12} & $\begin{array}{l}\text {-relapse prevention/ coping } \\
\text { planning (35) }\end{array}$ & & $\begin{array}{l}\text {-Greaves et al. } \\
(2011)\end{array}$ \\
\hline & & & $\begin{array}{l}\text {-provide of follow-up prompts } \\
\text { (27) }\end{array}$ & & $\begin{array}{l}\text {-Greaves et al. } \\
\text { (2011) }\end{array}$ \\
\hline & $\begin{array}{l}\text { - enhance abilities to } \\
\text { manage lapses and to } \\
\text { avoid relapses }\end{array}$ & $\begin{array}{l}13,14 \\
15,16\end{array}$ & $\begin{array}{l}\text {-training on beneficial } \\
\text { attributions }\end{array}$ & $\begin{array}{l}\text {-attribution (re- } \\
\text { )training }\end{array}$ & $\begin{array}{l}\text {-Wietkewitz \& } \\
\text { Marlatt (2004); } \\
\text { •Marlatt \& Gordon } \\
\text { (1985) }\end{array}$ \\
\hline & & $\begin{array}{c}13,14 \\
15,16 \\
17\end{array}$ & $\begin{array}{l}\text { - prompt self-monitoring (16) } \\
\text { (19) }\end{array}$ & $\begin{array}{l}\text { - } \log \text { respective food } \\
\text { diary }\end{array}$ & $\begin{array}{l}\text {-Carver \& Scheier } \\
(1998)\end{array}$ \\
\hline & $\begin{array}{l}\text { - enable abilities for } \\
\text { resumption }\end{array}$ & $\begin{array}{l}13,14 \\
15,16 \\
17\end{array}$ & $\begin{array}{l}\text { - provide feedback on } \\
\text { performance (19) }\end{array}$ & $\begin{array}{l}\cdot \log \text { respective food } \\
\text { diary }\end{array}$ & $\begin{array}{l}\text {-Greaves et al. } \\
\text { (2011) }\end{array}$ \\
\hline
\end{tabular}




\subsection{Appendix 6 Recruitment Letter}
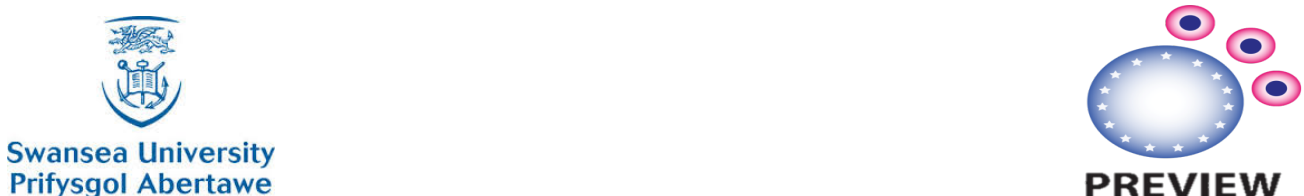

Swansea University
Prifysgol Abertawe

PREVIEW

Letter to parents

(Sent from schools following Swan-linx report)

Dear Parents

[Name of School] has recently taken part in a research project at Swansea University that has assessed the health and fitness of all the year 5 and 6 students. We have been made aware that many of our pupils would benefit from increasing their activity and improving their diet.

We would like to inform you about a program that is being run at Swansea University that aims to help children be more active and make better dietary choices.

Children aged 10 to 18 -years old across Europe are already taking part in the study. Our pupils can get involved too, and learn how to eat and exercise better, and take important steps against developing serious illnesses later in life.

When taking part, children and their parents will get professional guidance from exercise and nutrition scientists, exercise behaviour specialists, and paediatric health professionals.

Children can also win footballs, vouchers and will be given a pass to children's gym at the LC2.

If you would like more information about taking part contact Nils Swindell

Email:

Phone: 0 


\subsection{Appendix 7 Radio Advert}

\section{Script of radio advert to be played on Swansea Sound, Wave Radio}

MUSIC: VH470 Track 51/Just around the corner by M Ragan

GIRL:

BOY:

ANNO:

MUSIC BREAKS
My mum says I should eat healthy, why?

My dad says I should exercise more, what for?

If your child doesn't understand why they should eat healthy and exercise, maintaining a 'healthy' body weight becomes a problem for them.

Swansea University would like you and your child to take part in a study on the prevention of 'type 2' diabetes.

We'll provide a healthy lifestyle program so you can 'all' see the benefits of being active, eating a better diet and maintaining a healthy body weight.

All child participants receive a pass to the LC2 Gym.

To register call Swansea 123456. That's 123456 


\subsection{Appendix 8 Interview Questions}

\section{Children's questions}

- Has taking part in this program been a positive experience?

- Would you recommend this program to someone else?

- What would you say you have gained from taking part? (competency)

- Can you give me an example of something you have learnt?

- Can you give me an example of a behaviour that you have changes because of taking part in this program?

- Will your behaviours or you attitude towards these behaviours change now that the study is over?

- What will change now that the study has ended?

- How likely are you to carry on your healthy behaviours? Can you give me an example of a behaviour that you will continue?

- How do you feel about your own ability to continue with this behaviour? (self efficacy)

- During the study, we talked about self monitoring, do you currently use any methods of monitoring your own behaviour, goals or progress?

- Do you have goals that you are trying to achieve? (action planning/goal setting) Can you give me an example? Do you use SMART goals? If not how do you define your goals?

- How do you feel about your own ability to achieve these goals? (self efficacy)

- Did you find the process of setting SMART goals helpful?

- Have you experienced any relapses over the past few months?

- What would you say was the cause of the relapse? (attribution)

- How did you recover from this reaps? (goal adjustment)

- How are you effected by a relapse? How would you prevent it form happening again?

- Have you ever not achieved a goal you have set for yourself?

- How did you respond when this happened?

- Is there any feedback you would like to give us, anything you think we could have done differently to improve the program or make it a better experience for you?

\section{Parent's questions}

- Has taking part in this program been a positive experience for your child?

- Would you recommend this program to someone else?

- What has your child gained from taking part? (competency)

- Can you give me an example of something they have learnt?

- Can you give me an example of a behaviour they have changes because of taking part in this program?

- Do you think their behaviours will change now that the study is over?

- Will anything change now that the study has ended?

- How likely they to carry on their healthy behaviours?

- How confident do you think they are in their own ability to continue with their healthy behaviours? (self efficacy)

- During the study, we talked about self monitoring, do they use any methods of monitoring their own behaviour, goals or progress?

- Do they currently have goals that they are trying to achieve? (action planning/goal setting) Do they set SMART goals? If not how do they define theirgoals?

- How confident do you think they are in their own ability to achieve their goals? (self efficacy)

- Have they experienced any relapses over the past few months?

- What would you say was the cause of the relapse? (attribution)

- How did they recover from this reaps? (goal adjustment)

- How are you effected by a relapse? How would you prevent it form happening again?

- Have you ever not achieved a goal you have set for yourself?

- How did you respond when this happened? 
- Is there any feedback you would like to give us, anything you think we could have done differently to improve the program or make it a better experience for you? 


\subsection{Appendix 9 Transcriptions}

Child and parent perceptions, quotations from semi structured interviews ordered by theme and time point

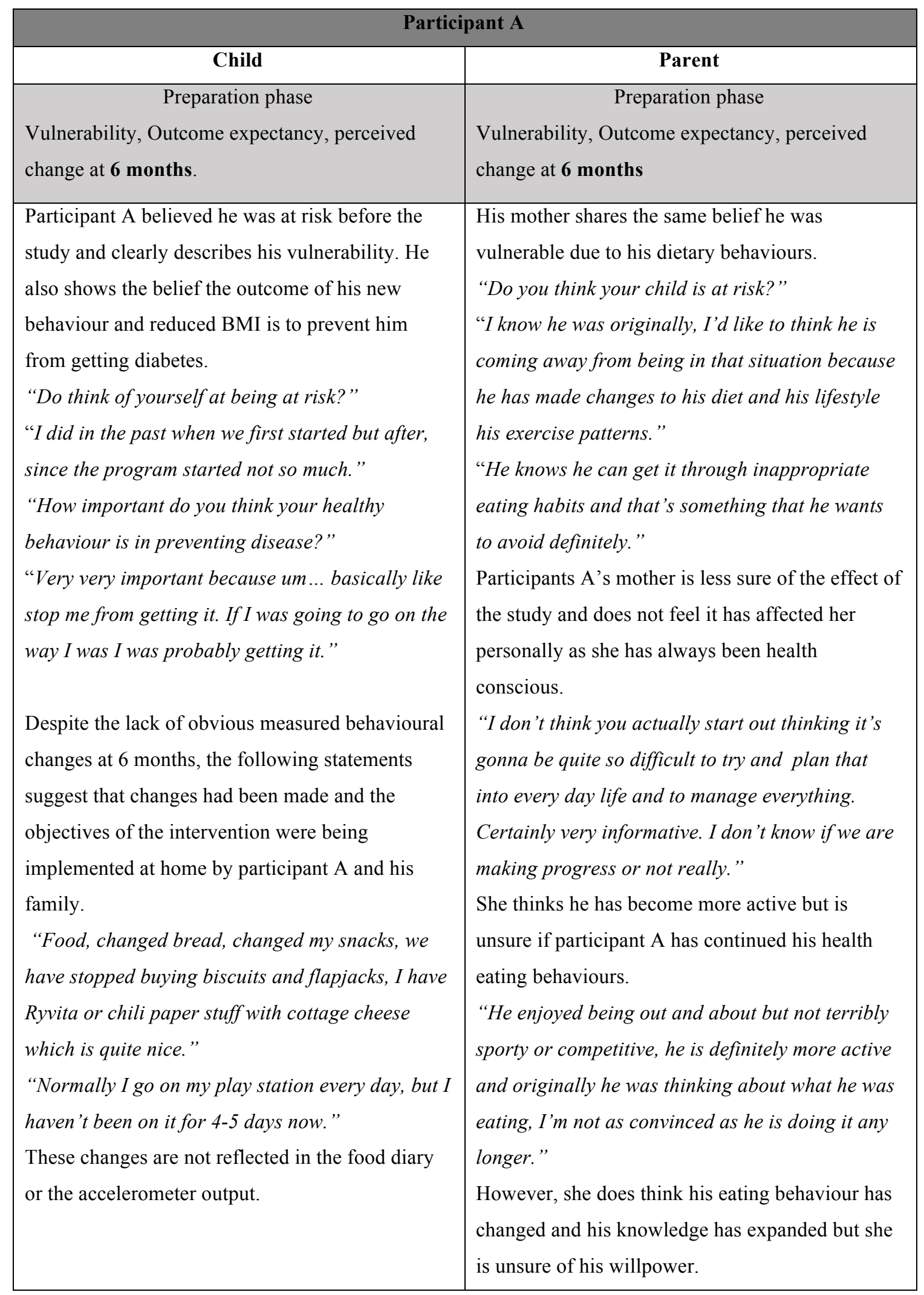




\begin{tabular}{|c|c|}
\hline & $\begin{array}{l}\text { "He is eating less than he was. His skills and } \\
\text { knowledge has definitely expanded Whether he } \\
\text { has the will power to stick to them all the time is a } \\
\text { different matter." }\end{array}$ \\
\hline Competency/Self-efficacy 6 months & Competency/Self-efficacy 6 months \\
\hline $\begin{array}{l}\text { Participant A demonstrated competency in } \\
\text { deciding his own food and when prompted } \\
\text { provides examples of healthy snacks he enjoyed. } \\
\text { "Yeah I think so, I'll have porridge for breakfast, } \\
\text { a sandwich or wrap for lunch and stir fry for } \\
\text { dinner." } \\
\text { "Snacks?" } \\
\text { "Carrots and dips, Ryvita with cottage cheese and } \\
\text { grapes" } \\
\text { Participant A also displays competency in } \\
\text { completing physical activities including an } \\
\text { exercise routine using equipment he keeps in his } \\
\text { room. } \\
\text { "In my room I got sit up bench, weights, I got like } \\
\text { a surf and practice board, I have my little } \\
\text { exercise, first do it with your hands and then your } \\
\text { back, so it's good." } \\
\text { He later shows us his equipment and explains his } \\
\text { routine demonstrating his confidence in his own } \\
\text { ability to perform the described exercises. }\end{array}$ & $\begin{array}{l}\text { Participants A's mum believes that he is confident } \\
\text { with the general healthy eating guidelines but that } \\
\text { the introduction of GI was too complicated. } \\
\text { "The GI thing I don't think he has grasped fully, } \\
\text { the principles are the same but I think it's become } \\
\text { too scientific for the kids." } \\
\text { However, she does demonstrate the belief that he } \\
\text { has got the ability to make healthy food choices if } \\
\text { he is motivated, and that his competency has } \\
\text { increased because of the study. } \\
\text { "How competent is your son regarding making } \\
\text { decisions about his diet and exercise?" "On a } \\
\text { scale of } 1 \text { to } 10 \text { about } 7 \text { probably. When we were } \\
\text { talking about healthy eating and the different } \\
\text { groups of foods he could see the balance there." } \\
\text { "Is he able to plan a healthy diet, since the } \\
\text { program? } \\
\text { Yes he is able to but not always motivated to "Is } \\
\text { "Yes definitely." }\end{array}$ \\
\hline Coping self-efficacy 12 months & Coping self-efficacy 12 months \\
\hline $\begin{array}{l}\text { At } 12 \text { months, the focus of the meetings moves } \\
\text { toward coping self-efficacy and the ability to } \\
\text { overcome setbacks. } \\
\text { Participant A shows how he can cope with } \\
\text { setbacks by focusing on his goals. } \\
\text { "How would you overcome this?" } \\
\text { "Um.... Probably think of how far everything has } \\
\text { changed since the start of the program and try to } \\
\text { motivate myself of doing something even if it's just } \\
\text { a 10-minute walk" } \\
\text { "What have you learnt from your relapse and how }\end{array}$ & $\begin{array}{l}\text { His mother also agrees that he is not heavily } \\
\text { affected by relapses and that he is able to put them } \\
\text { behind him and move on. } \\
\text { "He doesn't dwell on it- I'm almost inclined to } \\
\text { say what's to learn really, because we're all } \\
\text { human and we all recognize that we can't be } \\
\text { perfect all the time. I don't think he really } \\
\text { relapses in terms of physical activity. In terms of } \\
\text { food, he does on occasion, - the lesson learnt is } \\
\text { the recognition of it. That's the attitude he adopts, } \\
\text { he doesn't beat himself up over it." }\end{array}$ \\
\hline
\end{tabular}




\begin{tabular}{|c|c|}
\hline $\begin{array}{l}\text { did you recover from it?" } \\
\text { "Um.. that it happens to everyone and it's not } \\
\text { always a bad thing" } \\
\text { "Um.... I say probably getting back in the gym } \\
\text { and thinking about how fit and healthy I would be } \\
\text { because that really motivates me." } \\
\text { "Do you feel able to overcome it if you were to } \\
\text { have another relapse?" } \\
\text { "Yeah definitely" } \\
\text { "What would you do?" } \\
\text { "I would probably get help from my mum because } \\
\text { like she worked for me and it helps me a lot and I } \\
\text { would try my best to get back in the gym again } \\
\text { and eat healthy." }\end{array}$ & \\
\hline Competency Self-efficacy 24 months & Competency Self-efficacy 24 months \\
\hline $\begin{array}{l}\text { Participant A reflects on what he has gained form } \\
\text { taking part in the program and is confident he will } \\
\text { continue in the future. } \\
\text { "Actually I have gained a lot of information about } \\
\text { healthy diet and a little bit of fitness." } \\
\text { "I probably do more exercise" } \\
\text { "I've started making healthy meals before school } \\
\text { and everything." }\end{array}$ & $\begin{array}{l}\text { His mother believes he has gained a great deal and } \\
\text { has confidence in his own decisions about health. } \\
\text { "What do you think your child has gained from } \\
\text { taking part?" } \\
\text { "The confidence to know that the choices that he's } \\
\text { making are right, a commitment to activity, no } \\
\text { doubt about that and just really perhaps a } \\
\text { different way of thinking about his well-being I } \\
\text { guess. His health and his fitness." } \\
\text { "He'd have learned how to read food labels, he } \\
\text { would have learnt how to make healthy food } \\
\text { choices and about the level of activity that he } \\
\text { should be undertaking in order to live a healthy } \\
\text { lifestyle." } \\
\text { The following statement demonstrates her } \\
\text { perception of his competency, self-efficacy and } \\
\text { the autonomy regulating his health-related } \\
\text { behaviours. } \\
\text { "How confident do you think he is in his own } \\
\text { ability to continue with these healthy } \\
\text { behaviours?" } \\
\text { "Very confident I don't think even he would think }\end{array}$ \\
\hline Autonomy 6 months & Autonomy 6 months \\
\hline Participant A, is at secondary school and gets & His mother also perceives him to have a high \\
\hline
\end{tabular}


lunch form the canteen, as his mother works late he also makes dinner at home with the help of his older sister. Consequently, he has a high degree of independence around food choice.

"Yeah, I guess like, say in school like the first day I got back there was pizza and I chose chicken curry, and I buy water every day so .."

Are you allowed to choose the food you think is healthy? Do your parents buy what you choose? "Yeah, most of the times, it might be something different but as healthy"

It's good like getting home looking in the fridge and seeing all the healthy food. My mum always asks me what I want for shopping, instead of fizzy drink we will have sparkly lime/lemon water, " [Repat example]

Participant A can choose his own activities and lives near the park, playing fields and cycle path.

"Yeah, on my planner I put on 60 min playing football and I'll end up playing for 2 or 3 hours. Because I'm enjoying myself, I'm with my friends". [link to evidence accelerometer]

\section{Autonomy 12 months}

Participant A's autonomy has only improved since the last meeting as he can now go to the gym by himself.

"Going to the gym and there are always other ways to get there" degree of autonomy over his food choices

"When you really think about it, two third of meal

choices he makes he makes them outside the

home." [mother]

"may be because he is having to become more self-reliant to make his own food choices, making his own food, this has really come at the right time because it's point him in the right direction."

\section{Autonomy 12 months}

His mother states that his behaviour is largely down to him and that he is self-motivated.

"It's all down to him really. $80 \%$ if not more down to him. The rest is down to the interactions he has with family members around food and exercise and support. The exercise is an issue because if you say that I'd say about $100 \%$ well 95\% down to him because the majority of it he'll do self-motivated and he just go from here and do it. He does need transport on occasions to different places which is where other people's responsibility is come in as well."

"In terms offood he is making his own choices when I'm not around, that is the majority of the time other than the weekends."

\section{Action planning-Goal setting 6 months}

Participant A states that he finds setting goals
Action planning-Goal setting 6 months

Participant A's mother believes that he does not 


\begin{tabular}{|c|c|}
\hline 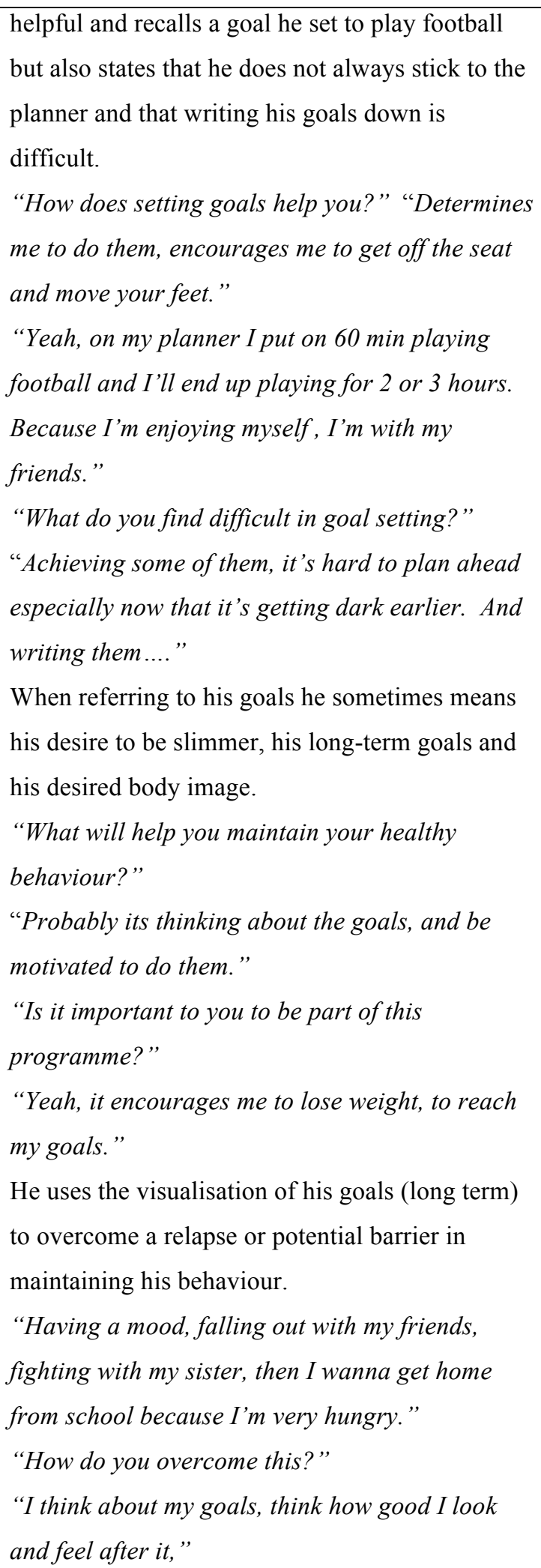 & $\begin{array}{l}\text { keep a written account of his goals and struggles } \\
\text { with his planner } \\
\text { "For participant A, writing the planner is a } \\
\text { massive effort, why does he have to do it?" } \\
\text { "He was fairly active anyway so from his } \\
\text { perspective he was thinking well I'm doing it } \\
\text { anyway why do I have to plan it?" }\end{array}$ \\
\hline $\begin{array}{l}\text { Action planning/Goal setting, } 12 \text { months } \\
\text { (also self-monitoring and contracting) }\end{array}$ & $\begin{array}{l}\text { Action planning/Goal setting, } 12 \text { months } \\
\text { (also self-monitoring and contracting) }\end{array}$ \\
\hline $\begin{array}{l}\text { Participant A can remember his behaviour change } \\
\text { contract but does not remember any details. } \\
\text { "Do you remember your behaviour change }\end{array}$ & $\begin{array}{l}\text { His mother agrees that he monitors his progress } \\
\text { through the changes he has seen but not with any } \\
\text { but not through any written methods, food diary, }\end{array}$ \\
\hline
\end{tabular}


contract?"

"Yeah I do remember that"

"Do you remember the goals and objectives that were on it?"

"I remember one of them which was to lose weight or to maintain my weight I honestly can't remember any."

It is apparent from the answers to previous questions that Participant $\mathrm{A}$ has got goals in his mind as he frequently refers to his goals, but he does not use a structured way of documenting his goals such as the SMART template.

"What was the last goal you set yourself?"

"It was for holidays to go to the gym once everyday"

"Um.. I think it was going to start in December to try and do 100 push ups a day and sit ups and I been setting a goal of training every other day for the $5 k$ because if I run one day I would always try and walk the next day."

"Does setting goals help you?"

Um.. I say it helps quite a lot because its something to aim to and it feel something you have to complete."

He has clear sporting aspirations and goes to the gym regularly to improve his performance. He often sates that he wants to do his best but does not have a structured way of self-monitoring. "I do weight myself sometimes and I do look at pictures from before and compare them to now." "What about your diet like how many vegetables you have eaten?"

"Um... no."

"Yeah, for performance say a number of activities I want to do or achieve in a match like the number of tackles or the number of ball carries."

"Um.. I think um probably by always trying to be my best."

"How do you know you have made progress"

"Um... the way my body feels" activity log or an app.

"Does your son monitor his own progress?"

"He does it visually because he looks at himself and he sticks his leg out or flex his muscles or contracting his muscle to see how big they are these days. So I guess it's incremental isn't it? He is proud of the changes in his body." 
"Ok so what about in the gym? Do you notice the weights that you are lifting are getting heavier?"

"Um... yeah because I always push myself" participant A receives feedback from the study personal, his family and friends and finds this a motivation to continue.

"Is it important to have feedback on you progress?",

“Um.. I feel it's quite important for me as it also makes me motivate me and makes me feel better about myself."

"Who do you receive feedback from?"

"You, family and some of my friends."

Despite valuing feedback from the study personnel, he does not feel there has been a negative impact of increased gaps between the meetings.

"How has the increased gaps between our visits influenced your healthy behaviours?"

Um... its influenced quite a lot because you have to keep on doing what you're doing for a longer time so it's going to help you in the future”.

"In some ways it might have been easier to forget about [the program] because you're not going to see us for 6 weeks. So do you think there's a factor of that?

"Um... na not really."

\section{Action planning Goal setting 24 months}

Participant A is still setting goals for himself but does not use a system to record them. When pushed he recalls what SMART goals are but he does not define his goals with the SMART principles.

"Do you have goals that you are trying to achieve?"

"Yes."

"Can you give me an example?"

"Like say training three times a week and going for two runs a weekend.

Eating more vegetables and proteins and less fatty

\section{Action planning Goal setting 24 months}

It is also his mothers perspective that although he has got clear sporting goals and aspirations he does not use structure way of monitoring his progress.

"I think probably subconsciously what he's doing. He's very committed to his goal around rugby.

Very very committed to his goal around rugby. So that's what he's working towards and I think having been taught those methods of motivating yourself certainly contributed. Whether he actually sits down and plans things out or not, I'd be quite doubtful of to be honest with you but I 


\begin{tabular}{|c|c|}
\hline $\begin{array}{l}\text { foods like chocolate and sweats.” } \\
\text { "When you say you eat less chocolate and less } \\
\text { fatty foods, how do you apply smart goals to that? } \\
\text { Because when you say less chocolate that's not } \\
\text { specific. How do you measure it? How do you } \\
\text { know it's achievable?” } \\
\text { "I cut down. Say if I had a whole bar, I only cut } \\
\text { down one block per week." }\end{array}$ & $\begin{array}{l}\text { just think it works because it's become a way of } \\
\text { life. I don't think he does anything formal in that } \\
\text { regard but on the other hand he's working } \\
\text { towards that goal." } \\
\text { His mother also believes that diet and exercise } \\
\text { have become such a way of life that he does not } \\
\text { have a need to plan out his goals as he did in the } \\
\text { beginning. } \\
\text { "He knows what the goals are that he wants to } \\
\text { achieve. He knows what he wants to improve. So } \\
\text { what he does is set himself exercise plans. So they } \\
\text { are specific goals, he can measure them, they are } \\
\text { achievable and he's doing them in a timely } \\
\text { fashion. I actually like to say it's because it's } \\
\text { become a way of life for him, the necessity to be } \\
\text { structured about it in terms of sitting down and } \\
\text { writing down and thinking them through properly } \\
\text { we've probably gone beyond that stage I would } \\
\text { have thought." }\end{array}$ \\
\hline & \\
\hline $\begin{array}{l}\text { Participant A lives with his mum who he sees as } \\
\text { role model } \\
\text { "she is my role model, somebody has to be your } \\
\text { role model, I don't live with my dad so she is my } \\
\text { role model." } \\
\text { He also has a strong sporting role model in his } \\
\text { father, although they do not live together he is a } \\
\text { presence and encourages the participation in sport. } \\
\text { "My dad is my role model in an active way } \\
\text { because he played rugby and he encouraged me a } \\
\text { lot to play sports and he played for the All Whites } \\
\text { St. Helens and he played against New Zealand." } \\
\text { Grandparents very helpful, they always take me } \\
\text { somewhere, they encourage me to do well in life, } \\
\text { they'll take me anywhere I want, they offer to take } \\
\text { me to rugby and everything." } \\
\text { Participant A also refers to his friends as a source } \\
\text { of motivation and compares himself to others } \\
\text { "looking at my friends and see how fit and healthy } \\
\text { they are, I couldn't do half the things they could }\end{array}$ & $\begin{array}{l}\text { She models healthy behaviours } \\
\text { "I always watch what I eat. I have always done } \\
\text { exercise one form or another, but it's trying to } \\
\text { encourage children to follow that example." } \\
\text { His mother does not think he talks to his friends } \\
\text { about being on the program but his friends are a } \\
\text { positive influence when it comes to physical } \\
\text { activity. } \\
\text { "I don't think he talks to his friends about it } \\
\text { [project], he has got 2-3 friends that he's told he } \\
\text { is doing this [project], one of whom is very sporty } \\
\text { anyway and that's a real benefit for him because } \\
\text { Tom would go off and do football in one direction } \\
\text { but now he is doing it with Participant A it's } \\
\text { really helped in that regard." } \\
\text { The following statement suggests that } \\
\text { participants' grandparents are on-board with } \\
\text { health eating messages and offer regular } \\
\text { reminders, however it also shown that he does not } \\
\text { share the information with his peers. }\end{array}$ \\
\hline
\end{tabular}




\begin{tabular}{|c|c|}
\hline $\begin{array}{l}\text { do, they would probably find it easy, so I wanna } \\
\text { be like them," } \\
\text { His body image has been a concern, he felt self- } \\
\text { conscious when going swimming and used this as } \\
\text { a motivation to start the program. } \\
\text { "Self-esteem has increased, in PE I used to cover } \\
\text { up, now I just get changed, I don't even care of } \\
\text { what other people think, so my confidence has } \\
\text { also changed" }\end{array}$ & $\begin{array}{l}\text { "My parents took Tom and participant A down to } \\
\text { the caravan and every now and again my mother } \\
\text { goes "ooh are you sure that is a healthy food?" } \\
\text { And participant A will just stare at them and go } \\
\text { "oh just be quiet." }\end{array}$ \\
\hline Social support 12 months & Social support 12 months \\
\hline $\begin{array}{l}\text { In keeping with the first meeting, participant A } \\
\text { mentions a number of positive social supports that } \\
\text { help him. His friends are a positive influence as } \\
\text { well as his family that motivate him to be more } \\
\text { active. } \\
\text { "Um ... support from my family and friends um.. } \\
\text { like everyone around you”, } \\
\text { "my friends are quite active as well so they } \\
\text { actually want to do something if I wanted to } \\
\text { something”. } \\
\text { "My family because they make me feel that I want } \\
\text { to go out or make me go out because if I don't go } \\
\text { out they will think my fitness will deteriorate or } \\
\text { something." }\end{array}$ & $\begin{array}{l}\text { "definitely. We ignore what Alice eats then, we } \\
\text { just get on with it. Because he actually doesn't } \\
\text { want to eat the same stuff as her" }\end{array}$ \\
\hline Maintenance 6 months & Maintenance 6 months \\
\hline $\begin{array}{l}\text { When asked if he is likely to continue with his } \\
\text { new healthy behaviours, participant A answers } \\
\text { with certainty and is looking forward to adding } \\
\text { further activity to his schedule: } \\
\text { "Yeah, a lot likely- I'm starting rugby next week } \\
\text { and my aunty is getting a surf board soon so we } \\
\text { are gonna go surfing." }\end{array}$ & \\
\hline Maintenance 12 months & Maintenance 12 months \\
\hline $\begin{array}{l}\text { Participant A is confident he is maintaining his } \\
\text { healthy behaviours } \\
\text { "Yeah, I'm always trying to keep active every } \\
\text { hour and always thinking about my food of what } \\
\text { is healthy and looking at the calories." Um.... I }\end{array}$ & $\begin{array}{l}\text { His mum agrees that he has maintained his } \\
\text { behaviour change particularly in respect to } \\
\text { physical activity. It is a way of life now and she } \\
\text { sees it continuing. } \\
\text { "Has he maintained his healthy behaviour?" }\end{array}$ \\
\hline
\end{tabular}


always choose the healthiest option um.. I always think it will help me."

"Um... in Cyprus I got asked if I wanted a pudding and I went to the fruit first because if I'm still hungry after that maybe I'll have it but that will probably fill me up."

He is also confident he will carry his healthy behaviours into the future, describing his schedule of getting up early to go to the gym everyday whilst on holiday as an example of his determination.

"Because I really want to be really fit and healthy, I know that's going to help towards it. When I'm in the gym or when I get up really early to go to the gym because it helps burn calories so um... we always try and get up early on holiday."

Maintenance 24 months
"Will your behaviours or your attitude towar
these behaviours change now that the study i
over?"
Child "No.
"Will anything change now that the study is
ending"

Child: "I don't think anything will change."
"Yeah definitely, the exercise is fundamental to everything that he does now. So he doesn't even think twice about it."

"In terms of eating we have just come back from an all-inclusive holiday and he would come back [from the self-service buffet] with salad and meat on his plate. And the difference we saw in him this year compared with last year was incredible, so much more confident in himself, he is just a happier kid really. It's really lovely to see."

"How likely is your child to carry on with the behaviour change?"

"100\% he is likely to keep it I have no doubt about it at all.

Because it's ingrained in what he is doing every day."

(1)

"Will your child's behaviours or attitude towards

these behaviours change now that the study is over?

No. I think he will just carry on. No doubt about that"

"Will anything change now that the study is ending"

I think he'd be relieved not to have to do those tests again. But on the other hand no I think the principles of what this study taught him are just completely embedded and will carry on.

\begin{tabular}{l|l|}
\multicolumn{1}{|c|}{ Relapse 12 months } & \multicolumn{1}{c|}{ Relapse 12 months } \\
$\begin{array}{l}\text { Participant A acknowledges that sometimes it is } \\
\text { hard to stick to his healthy behaviours and }\end{array}$ & $\begin{array}{l}\text { "he knows where he wants to be, what his goals } \\
\text { are, so yeah he might have a couple of chocolate } \\
\text { attributes relapses to special occasions and } \\
\text { describes his own laziness as a barrier. }\end{array}$ \\
$\begin{array}{l}\text { Child: "Well I say, what I was doing last year for } \\
\text { Christmas and after that it just deteriorated and I }\end{array}$ & stuff as I used to, so it's not in the house anyway." \\
wasn't focusing on my diet or anything." \\
"Like, laziness or yeah probably when I have had \\
a really bad day I basically go up to my room stay
\end{tabular}




\begin{tabular}{|c|c|}
\hline $\begin{array}{l}\text { up there and just eat anything but yeah and } \\
\text { feeling like really lazy which isn't all the time but } \\
\text { it can happen sometimes." } \\
\text { Visualization and self-talk } \\
\text { Participant A explains that he has used } \\
\text { visualization and self-talk while in the gym and } \\
\text { while trying to avoid food temptations. } \\
\text { "When I'm in the gym if I feel really tired or } \\
\text { anything o always visualize what the outcome } \\
\text { would be in the future." } \\
\text { "I'm always trying to do more by saying come on } \\
\text { push yourself further." } \\
\text { "Probably say to myself in the head don't give In } \\
\text { to anything, don't eat anything ridiculous }\end{array}$ & \\
\hline Relapse 24 months (Coping self-efficacy) & Relapse 24 months (Coping self-efficacy) \\
\hline $\begin{array}{l}\text { Participant A explains how he experienced a } \\
\text { relapse and was able to deal with it and how he } \\
\text { would prevent it from happening in the future. } \\
\text { "After my birthday. I just went off track for about } \\
\text { a week." } \\
\text { "I felt sloppy and lazy. I felt like my fitness } \\
\text { dropped down." } \\
\text { "How did you recover from the relapse?" } \\
\text { "I let it go." } \\
\text { "How would you prevent it from happening } \\
\text { again?" } \\
\text { "I think I'll try and have less fatty foods around } \\
\text { me. Or I will try and resist the temptation." }\end{array}$ & $\begin{array}{l}\text { His mother does not think there have been any } \\
\text { relapses of not and that } \\
\text { "When you look back a year you'd think there are } \\
\text { periods where he went through times where he } \\
\text { wasn't as commit there's no doubt about it. But } \\
\text { generally speaking over the last month I wouldn't } \\
\text { have said so. Not really", }\end{array}$ \\
\hline
\end{tabular}

\begin{tabular}{|c|c|}
\hline \multicolumn{2}{|c|}{ Participant B } \\
\hline Child & Parent \\
\hline $\begin{array}{c}\text { Preparation phase } \\
\text { Vulnerability, Outcome expectancy, perceived } \\
\text { change at } 6 \text { months. }\end{array}$ & $\begin{array}{c}\text { Preparation phase } \\
\text { Vulnerability, Outcome expectancy, perceived } \\
\text { change at } 6 \text { months }\end{array}$ \\
\hline $\begin{array}{l}\text { Participant B is aware of his vulnerability to } \\
\text { diabetes and he demonstrated that he knows the } \\
\text { outcome of his physical activity and dietary }\end{array}$ & $\begin{array}{l}\text { His Grandmother is all too aware of his } \\
\text { vulneranility to diabetes and associates risk } \\
\text { reduction with an outcome of diet and exercise. }\end{array}$ \\
\hline
\end{tabular}




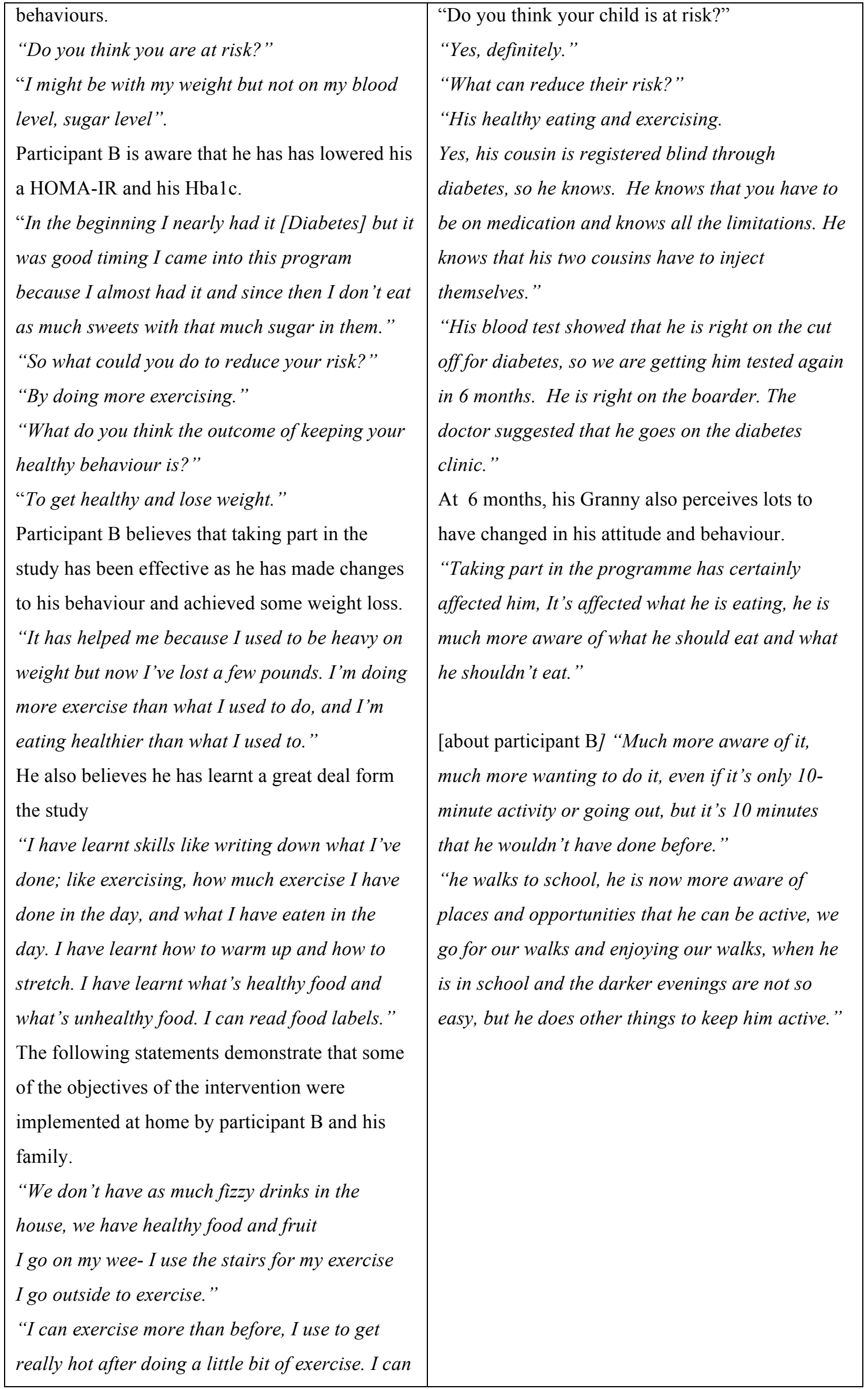


now do loads of exercise and I only sweat a little bit. I'm eating healthier food. And I now read food labels. I used to just pick up stuff without looking at food labels."

"I'm not drinking as much fizzy drinks-I gave up crisps, chocolate and Jam, difficult to be more active because I have to go outside and be active."

\section{Competency/Self-efficacy 6 months}

Participant B describes himself as confident to choose the right food and do exercise.

"I am very confident to pick the right food and exercise."

He demonstrates this ability to choose the right food in the following sentence as well as the ability to monitor his own dietary intake

"Yes- like in school they have fish and chips, but I choose something like pasta and healthy options."

"I have 5 a day-an apple, a banana in my packed lunch, strawberries, few veg with my meal: carrots, green beans, peas and sweet corn."

He is also able to plan 60 minutes of physical activity and his granny believes he is performing it too.

"Yes-I walk to school and back and that's half an hour, I play football for a quarter of an hour and I play on my wee for a quarter of an hour." He demonstrated confidence in his own ability when describing what he has learnt

"I have learnt skills like writing down what I've done; like exercising, how much exercise I have done in the day, and what I have eaten in the day. I have learnt how to warm up and how to stretch. I have learnt what's healthy food and what's unhealthy food. I can read food labels."

\section{Coping self-efficacy 12 months}

Participant B believes he is able to overcome relapses and describes how he regains his focus.

\section{Competency/Self-efficacy 6 months}

Hi Grandmother also believes he as able to perform activies on his own.

"Is he able to perform the planned activity?" Yes, on most times he does, if he doesn't he changes it then. If it's raining he doesn't want to go out and he will do something else." he is doing it (food labels) he will actually look at it, and I bought some drinks and he said have you looked at the labels? He is looking at labels. He said you know I have not been eating all those sweet things, I don't really miss them.

\section{Coping self-efficacy 12 months}

His grandmother describes over-coming a relapse as returning from him mothers house and they plan 


\begin{tabular}{|c|c|}
\hline $\begin{array}{l}\text { "I just remembered about this [the program], I } \\
\text { just remembered that I wanted to get fit so I just } \\
\text { went back to exercising and eating healthy, } \\
\text { remember my friends to go out with them." } \\
\text { "I'm really motivated to go out with friends", } \\
\text { "I just think overweight people eat McDonalds } \\
\text { and things. If I think of that then I will eat } \\
\text { healthy foods." } \\
\text { He has also developed a way of limiting his time } \\
\text { spent playing computer games. } \\
\text { "My Xbox switches off after a little while" } \\
\text { "Does it?" } \\
\text { "Yes" } \\
\text { "How long?" } \\
\text { "An hour." } \\
\text { This is an example of self-monitoring as well as } \\
\text { his ability to overcome his temptation to be } \\
\text { sedentary. He is confident he could overcome } \\
\text { another relapse. } \\
\text { "Would you overcome it if you had another } \\
\text { relapse?" } \\
\text { "Yes," "I would just think of football, go outside } \\
\text { and play football." }\end{array}$ & $\begin{array}{l}\text { together to restart their healthy routines. } \\
\text { "I suppose when he comes back into this } \\
\text { environment, he is encouraged, we talk about it, } \\
\text { we say shall we do this or shall we do the other } \\
\text { and he makes the choices and he's back on track } \\
\text { again." }\end{array}$ \\
\hline Competency Self-efficacy 24 months & Competency Self-efficacy 24 months \\
\hline $\begin{array}{l}\text { Participant B describes himself as more } \\
\text { confident and when he explains his activities } \\
\text { "I have played more football outside the house, } \\
\text { being more confident." } \\
\text { "Give me an example of a behaviour that you } \\
\text { will continue?" } \\
\text { Playing with my friends all the time. } \\
\text { "How many times a week?" } \\
\text { "10 times, I play in school as well." } \\
\text { "Anything else?" } \\
\text { "Eating healthily. Instead of eating crisps, I eat } \\
\text { fruit." } \\
\text { What will change now that the study is ending? } \\
\text { "I'll lose more weight. Gain even more } \\
\text { confidence" }\end{array}$ & $\begin{array}{l}\text { His grandma perceives him to be confident in his } \\
\text { own ability to continue with his behaviour. } \\
\text { How confident do you think he is in his own ability } \\
\text { to continue with these healthy behaviours? } \\
\text { "Yes because he's the one that is controlled it } \\
\text { really. He controls it more than what I do. Very } \\
\text { much up there." } \\
\text { His Grandmother can't put it down to specific } \\
\text { behaviours that have changed but say that his } \\
\text { attitude and awareness about diet and exercise } \\
\text { have completely changed. } \\
\text { "Yeah. I can say the generally he is more aware of } \\
\text { being active. He is more aware of what to eat. I } \\
\text { can't put it down to more than that because it } \\
\text { covers it all, doesn't it? He is more aware that yes } \\
\text { he should be doing that exercise, he is aware that }\end{array}$ \\
\hline
\end{tabular}




\begin{tabular}{|c|c|}
\hline $\begin{array}{l}\text { his own ability to continue independently after } \\
\text { the study. }\end{array}$ & $\begin{array}{l}\text { yes he has been sitting down for too long he should } \\
\text { be doing something." }\end{array}$ \\
\hline Autonomy 6 months & Autonomy 6 months \\
\hline $\begin{array}{l}\text { Participant B is the youngest of the group and } \\
\text { has most of his meals at home or at school. } \\
\text { "Are you allowed to choose the food you think is } \\
\text { healthy" } \\
\text { "Yes- not always" } \\
\text { He displays the autonomy to make health food } \\
\text { choices of his own fruition at school and at } \\
\text { home. } \\
\text { "Yes- like in school they have fish and chips, but } \\
\text { I choose something like pasta and healthy } \\
\text { options." } \\
\text { "I can make healthy food and snacks at home- } \\
\text { healthy bread sandwich with beef, with butter." }\end{array}$ & 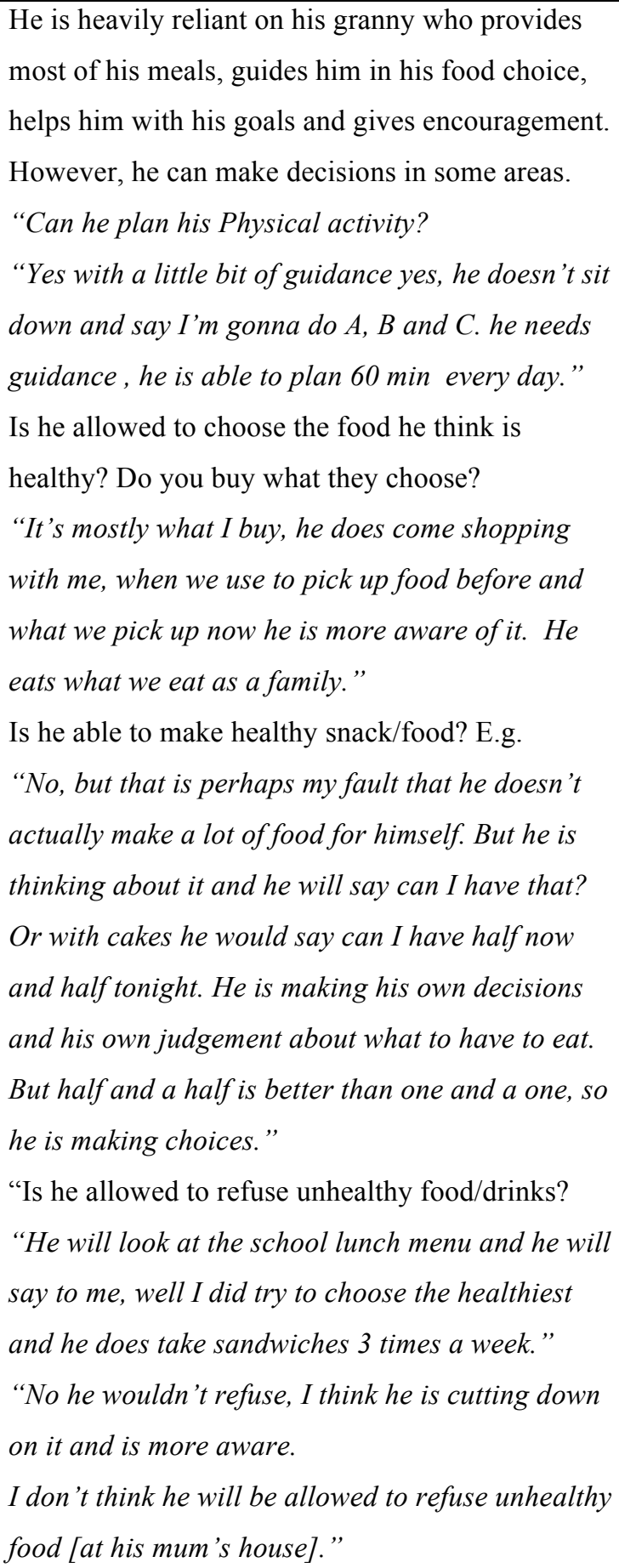 \\
\hline Autonomy 12 months & Autonomy 12 months \\
\hline $\begin{array}{l}\text { Moving to secondary school has changed his } \\
\text { environment his social influences and giving him } \\
\text { greater independence over his behaviours. He has } \\
\text { started going out to play football with friends and }\end{array}$ & $\begin{array}{l}\text { I told him to tell his friends that granny needs } \\
\text { walking and he's keeping her company. } \\
\text { His biggest social influence is still his gran. } \\
\text { "Granma has the biggest role. His main support }\end{array}$ \\
\hline
\end{tabular}




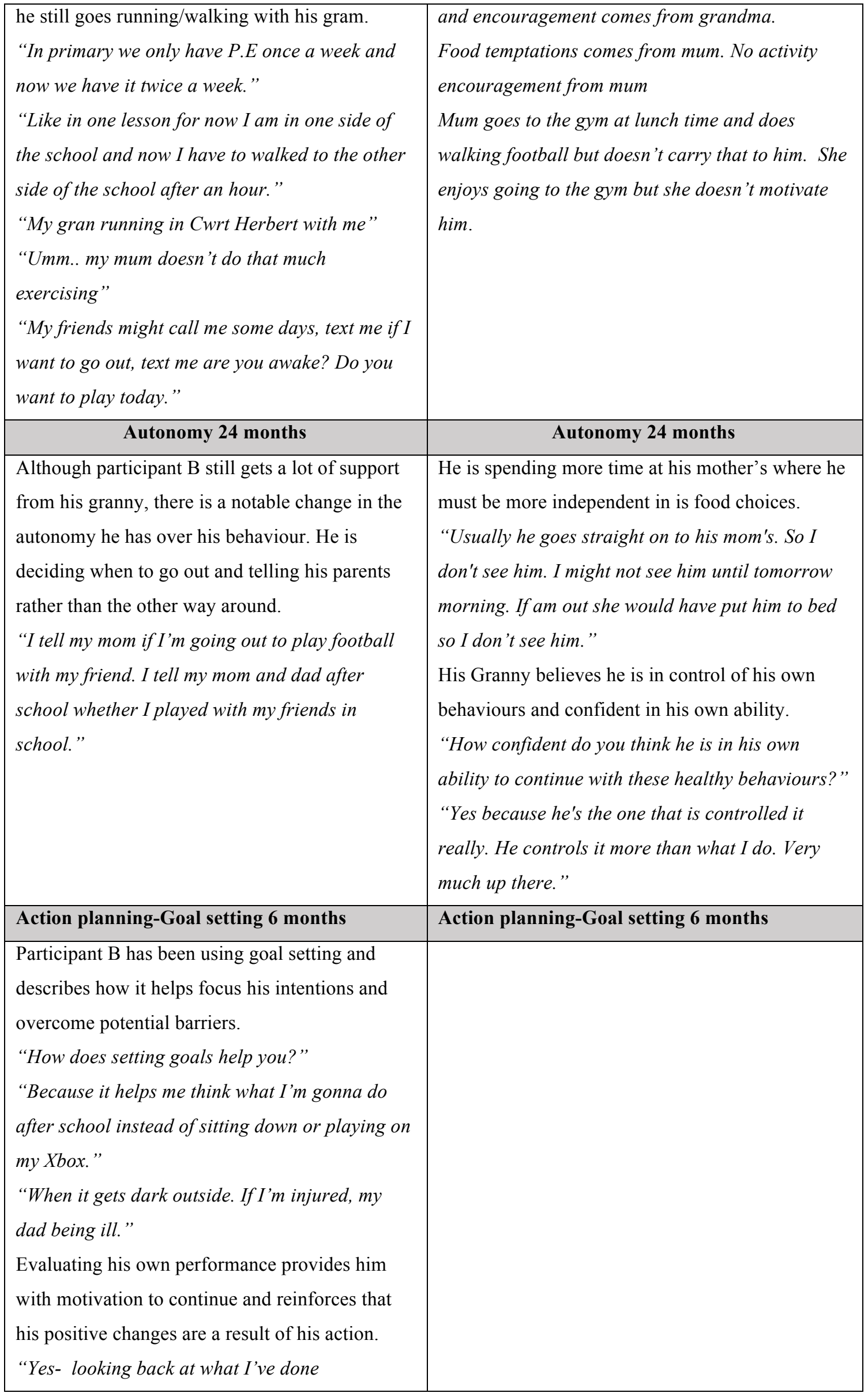


encourages me- like I have walked to school, I've played football."

"What I used to be like and what I am now-I like that change."

Action planning/Goal setting, 12 months (also self-monitoring and contracting)

Participant B remembers his behaviour change contract and some of the long-term goals but he doesn't remember any specific behaviours he wished to change or short term goals.

"Do you remember what your goals and objectives were on that contract?"

"To get fit, to lose weight, eat healthily"

When asked how he recodes his goals or reminds himself of his goals he gives some good ideas but they are not things he practices now.

"I keep a note on my fridge to keep fit"

"Have you?"

"I could."

Using clothe sizes as a goal is not specific as it does not address a behaviour, it is measurable although whether something fits or not could be subjective, the participant is still growing so this goal may not be realistic or achievable in the long term. There was no time frame for this goal. He also sets exercise goals in his mind.

"When I'm walking In Cwrt Herbert I think the next time I will do 4 times around Cwrt Herbert and the next time do 5 times until I can go around Cwrt Herbert 6 times."

This goal is not structured, written or discussed. However, it does roughly follow the SMART principles and is a progressive target that provides a focus.

Participant B is monitoring his behaviour by timing his laps around the park and trying to improve his time in line with FITT principles the children learnt in the study. He does not monitor his dietary intake but is very aware of his physical changes and cloth sized as mentioned
Action planning/Goal setting, 12 months (also self-monitoring and contracting)

His Grandmother can't remember his goals “I can't remember his goals and objectives."

Although they do not remember the written goals and objectives, together they do set verbal goals such and the plan for the holiday and setting targets for clothe sizes is a motivation.

"Communicating the goals and objectives with parents-talk about them, then go on a walk."

"Because on the holidays we said come on let's do it. This is the fittest 6-weeks holiday that he's ever had."

"he is delighted to buy the clothes that he likes and they fit him. He doesn't want to get any bigger than he is."

"His mum bought him his first hoody a large.

Within a few weeks going to school and running up and down, he'd lost some weight and the hoody looked big on him, so we bought a size medium.

He's got a goal in his mind that he doesn't want to go back to his previous weight and that keeps him motivated."

"For example if his goal is to shrink into a size medium that would help him."

"does he monitor his own behaviour?"

"Yes- he uses his stop watch for exercising so he is monitoring his time. Not sure about his diet."

"How do you know if your child has made progress?"

How many times he is exercising and also his time is getting shorter which means he is doing it faster. 
above.
"How do you know you made progress?"
"Getting taller and thinner, stronger muscles"
"do you monitor your own behaviour?"
"One day we will walk around Cwrt Herbert [a
local park] and write down how long it took us
on the sheet.
So I get my phone and get a timer and time how
long I been exercising for and stop it at the end
.... To see if I was quicker."

Action planning Goal setting 24 months

Participant B does not appear to be setting clear goals although he has got goals in his mind and has an active schedule, he is unable to recall what SMART goals are.

Do you have goals that you are trying to achieve?

"Yes"

"Can you give me an example?"

"Playing football after school."

"Any other activity you do?”

"Hiking or scouts."

Do you plan for that?

"The scouts plan us. They plan every time we are going to do it."

"What do you do? Do you plan any activity? You plan football, do you plan anything else?"

"Not really."

"Do you plan anything with your grandmother?"

"Going on walks"

\section{Action planning Goal setting 24 months}

His Granny is clearer that they have got goals which they have discussed verbally and write down, the goals are specific, measurable, achievable and time bound.

"Yes. Because we have gone away, his goal is not to put on and to lose some [weight] which he has been doing. And then after this holiday, there will be more goals."

This implies that they are weighing him which he has not disclosed. Basing goals on weight loss may not be achievable in the long term because of his age and continuing to grow [participants were not given weight loss goals during the intervention]. There is also a reward system between him and his granny.

How do you define these goals? Is it something you write down?

"Yes. Something you know he is going to do and we are always working towards something. Okay it's the holiday now and the next one will be October half term, we will work on someone October half term."

"So it has a reward for achieving that goal." "Yeah." "Is there a timeline for it?"

"Yes. Yes we are going on holidays and if you do... he was going to sit on the inner side 


\begin{tabular}{|c|c|}
\hline & $\begin{array}{l}\text { otherwise I'm having this for me to sit. But it } \\
\text { was sort of you've got to lose it before I book } \\
\text { it and yes he did. And then it was you got to } \\
\text { keep maintaining this otherwise I'm sitting by } \\
\text { the window and not you. And that's a reward. } \\
\text { Because we sit in three in a row because we } \\
\text { go with his mom and myself and him. So he } \\
\text { set a goal." }\end{array}$ \\
\hline Relatedness/social support 6 months & Relatedness/social support 6 months \\
\hline $\begin{array}{l}\text { Participant B's mother and one of his cousins are } \\
\text { type } 2 \text { diabetic, so he is familiar with its effects } \\
\text { and complications. His father and grandmother } \\
\text { offer positive influence regarding diet and } \\
\text { physical activity behaviours. } \\
\text { "Daddy helps me by playing football with me, } \\
\text { mummy reminds me to play football with my dad. } \\
\text { She reminds me to go on my wee,." } \\
\text { "How do you relate to your grandma?" } \\
\text { "She helps me a lot, she encourages me and she } \\
\text { helps me choose the right thing to eat." } \\
\text { He mentions his friends as a positive influence } \\
\text { but also refers to being bullied at school } \\
\text { suggesting his peers also represent a source of } \\
\text { ridicule or unhappiness. } \\
\text { "I am still friends with my friends, they're } \\
\text { helping me by saying I look better than I used to } \\
\text { and I'm better at football than I used to be. They } \\
\text { encourage me." } \\
\text { "If I get bullied in school I can talk about it with } \\
\text { my family and in the meetings, I have support of } \\
\text { my gran, my mum and dad, my friends, you and } \\
\text { Nils. }\end{array}$ & $\begin{array}{l}\text { Participant B lives with his Grandma and grand } \\
\text { dad, they eat together daily and they all eat the } \\
\text { same things. } \\
\text { "He knows we are eating what we must be eating } \\
\text { and he knows that the changes we have made are } \\
\text { minor really, we always have porridge for } \\
\text { breakfast so it's really easy because he will have } \\
\text { the same as me. We have gone over to pasta, well } \\
\text { we all eat pasta. And we have changed whole } \\
\text { seeded bread. We have found the changes easy to } \\
\text { adapt to." } \\
\text { Participant B has found it difficult to stick to } \\
\text { healthy behaviours when staying at his mother's } \\
\text { because of the temptations and foods offered to } \\
\text { him. } \\
\text { "How does he relate to you and other family } \\
\text { members?" } \\
\text { "Because of the situation in his life he actually } \\
\text { lives with me. He is getting much better in saying } \\
\text { no I don't want it thank you [if the food that's } \\
\text { being offered is unhealthy]." } \\
\text { "Food temptations comes from mum. No activity } \\
\text { encouragement from mum". } \\
\text { al }\end{array}$ \\
\hline Social support 12 months & Social support 12 months \\
\hline
\end{tabular}




\begin{tabular}{|c|c|}
\hline $\begin{array}{l}\text { Participants B mentions his friends on many } \\
\text { occasions as they are a source of motivation and } \\
\text { offer him feedback on his progress. Having } \\
\text { friends is very important to him and appears to } \\
\text { be a new development since the first meeting. } \\
\text { "My friends might call me some days, text me if I } \\
\text { want to go out, text me are you awake? Do you } \\
\text { want to play today." } \\
\text { "It means that I might get more friends later on } \\
\text { like one Saturday when we walked around Cwrt } \\
\text { Herbert there were } 2 \text { men running who are there } \\
\text { every Saturday and they said they want to see us } \\
\text { there every Saturday." } \\
\text { He has made a verbal contract with the two } \\
\text { runners and this has motivated him to be at the } \\
\text { park every week. }\end{array}$ & $\begin{array}{l}\text { The runners offer social support with friendly } \\
\text { advice that was remembered by both the } \\
\text { participant and his Granny. } \\
\text { "We remembered what the two runners said: they } \\
\text { said you have a top drawer full of excuses!! Close } \\
\text { that drawer. He said close it, don't open it because } \\
\text { you will have top drawer full of excuses." }\end{array}$ \\
\hline Maintenance 6 months & Maintenance 6 months \\
\hline $\begin{array}{l}\text { participant B is proud of what he has achieved } \\
\text { and believes he will continue with his } \\
\text { behaviours. } \\
\text { "I will keep on doing it.- people (my friends in } \\
\text { school) eating unhealthy food' drinking } \\
\text { unhealthy drinks- I get tempted but not as much } \\
\text { as I used to." } \\
\text { Vulnerability } \\
\text { Participant B is aware of his vulnerability to } \\
\text { diabetes or complications related to his weight } \\
\text { later life. He has got type } 2 \text { diabetes in the family } \\
\text { and knows the potential complications. This } \\
\text { offers a point of focus for him as he does not } \\
\text { want this to happen to him. } \\
\text { What will help you maintain your healthy } \\
\text { behaviour? } \\
\text { "Thinking about diabetes and what it does to } \\
\text { you" } \\
\text { Is it important to you to be part of this } \\
\text { programme? Why is } \\
\text { as ill as one of my cousins are" }\end{array}$ & $\begin{array}{l}\text { His Granny is not certain but hopes he will } \\
\text { continue. } \\
\text { "I hope he is, for his sake, I hope it's not because } \\
\text { he knows he is coming down to the university [to } \\
\text { the project]. I hope that is not his motivation. It is } \\
\text { to be eating healthier, I hope it is. I think and I } \\
\text { hope we are educating him that this is the right } \\
\text { road to go. It's every week he is losing a little bit." }\end{array}$ \\
\hline
\end{tabular}




\begin{tabular}{|c|c|}
\hline Maintenance 12 months & Maintenance 12 months \\
\hline $\begin{array}{l}\text { Participant B is maintaining and progressing his } \\
\text { physical activity, He also believes his diet has } \\
\text { improved. } \\
\text { "Like one week we got to the field and park once } \\
\text { or twice and now we going to the field } 4 \text { times." } \\
\text { "I used to eat McDonalds every week and now I } \\
\text { don't eat as much I eat it like once every month.. } \\
\text { Um.. I eat more fruit and vegetables." } \\
\text { He is determined to continue with his positive } \\
\text { change and shown his determination by going } \\
\text { gout to play football even if it is raining. } \\
\text { "I'm really determined to get fitter and taller } \\
\text { and to lose weight- } \\
\text { When it is raining and I want to go out and play } \\
\text { football with my friends." } \\
\text { He describes potential barriers as his temptations } \\
\text { for fast food and playing computer games when } \\
\text { he is tired. } \\
\text { "Eat Mcdonalds and not healthy foods." "I just } \\
\text { sit down and play with my xbox"” }\end{array}$ & $\begin{array}{l}\text { Participant B has made great progress meaning he } \\
\text { can now fit in smaller cloth sizes. This is a great } \\
\text { motivation and source of encouragement. His } \\
\text { progress is clearly visible by his appearance and he } \\
\text { receives positive feedback. } \\
\text { "Because he wants to lose weight. He doesn't want } \\
\text { to get any bigger than what he is and he is } \\
\text { delighted to buy the clothes that he likes and they } \\
\text { fit him. He doesn't want to get any bigger than he } \\
\text { is." } \\
\text { "In all his attitude, he is determined not to eat too } \\
\text { many rubbish, and he does want to exercise. He } \\
\text { does a mile in } 20 \text { minutes which is quite good." } \\
\text { He is se } \\
\text { "He feels fitter and I think it's all the } \\
\text { encouragement that everybody gives him. He can } \\
\text { see it, it's visual, he can see "I've done that"." } \\
\text { Barriers to maintaining healthy behaviours are his } \\
\text { grandmother's busy schedule and staying at his } \\
\text { mother's which offers food temptations and } \\
\text { doesn't support him in exercising. This is closely } \\
\text { related to his autonomy and reliance on his } \\
\text { grandmother. } \\
\text { "Mainly the environment- mum buying unhealthy } \\
\text { food bought pasty" }\end{array}$ \\
\hline Maintenance 24 months & Maintenance 24 months \\
\hline
\end{tabular}




\begin{tabular}{|c|c|}
\hline $\begin{array}{l}\text { Relapse } 12 \text { months } \\
\text { Participant B acknowledges that he has } \\
\text { experienced relapses when he is tired, or tempted } \\
\text { by unhealthy foods. } \\
\text { "Just when I don't feel like exercising or eating } \\
\text { right foods } \\
\text { "When I feel tired um.... Relaxed, bored" I just } \\
\text { sit down and play with my xbox" } \\
\text { Visualization and self-talk } \\
\text { Participant B, uses self-talk to distract himself } \\
\text { from temptations or to negative thoughts. He } \\
\text { visualizes himself in the future as the kind of } \\
\text { person he would like to be and he visualizes } \\
\text { playing football or rugby. } \\
\text { "Yesterday morning my arm was hurting and I } \\
\text { had negative thought thinking don't do any more } \\
\text { exercise because you are tired but I kept on } \\
\text { of exercise" } \\
\text { [self talk]"Stop eating more, stop thinking of } \\
\text { Mcdonalds and things" }\end{array}$ & $\begin{array}{l}\text { "Will your behaviours or your attitude towards } \\
\text { these behaviours change now that the study is } \\
\text { over? } \\
\text { "No. Because I won't let it even if he thinks. I } \\
\text { don't know what he thinks but I won't let it } \\
\text { because it's part of our conversation. It's part of } \\
\text { our everyday living. Have you done? Have you } \\
\text { had this or are you going to and that's what we } \\
\text { are having. It's a more of a general way of life. } \\
\text { That won't go away. It will always be here." } \\
\text { What will change now that the study is ending? } \\
\text { "No I don't think it will change. We will still be } \\
\text { doing what was recommended because you can't } \\
\text { force it, it was all recommendation. This is what } \\
\text { you should be doing; this is what you should be } \\
\text { eating. It's all recommendation and that will be } \\
\text { carrying through. And as I said not that I won't let } \\
\text { it, it's just that it is part of our conversations } \\
\text { 'Yes obviously he has some days that he doesn't } \\
\text { do much and I don't want to do it with him either." }\end{array}$ \\
\hline
\end{tabular}




\begin{tabular}{|c|c|}
\hline $\begin{array}{l}\text { [About having a goal] "It helps me to exercise, it } \\
\text { gives me an image in my head of who I want to } \\
\text { be." } \\
\text { Can you explain what you visualize? } \\
\text { "A man who is tall and thin." } \\
\text { "And I think about what will fit people do." } \\
\text { "just think of the Olympics, so think one day I } \\
\text { could be up there } \\
\text { playing rugby and things football, rugby one day } \\
\text { I could be playing rugby or football." }\end{array}$ & \\
\hline Relapse 24 months (Coping self-efficacy) & Relapse 24 months (Coping self-efficacy) \\
\hline 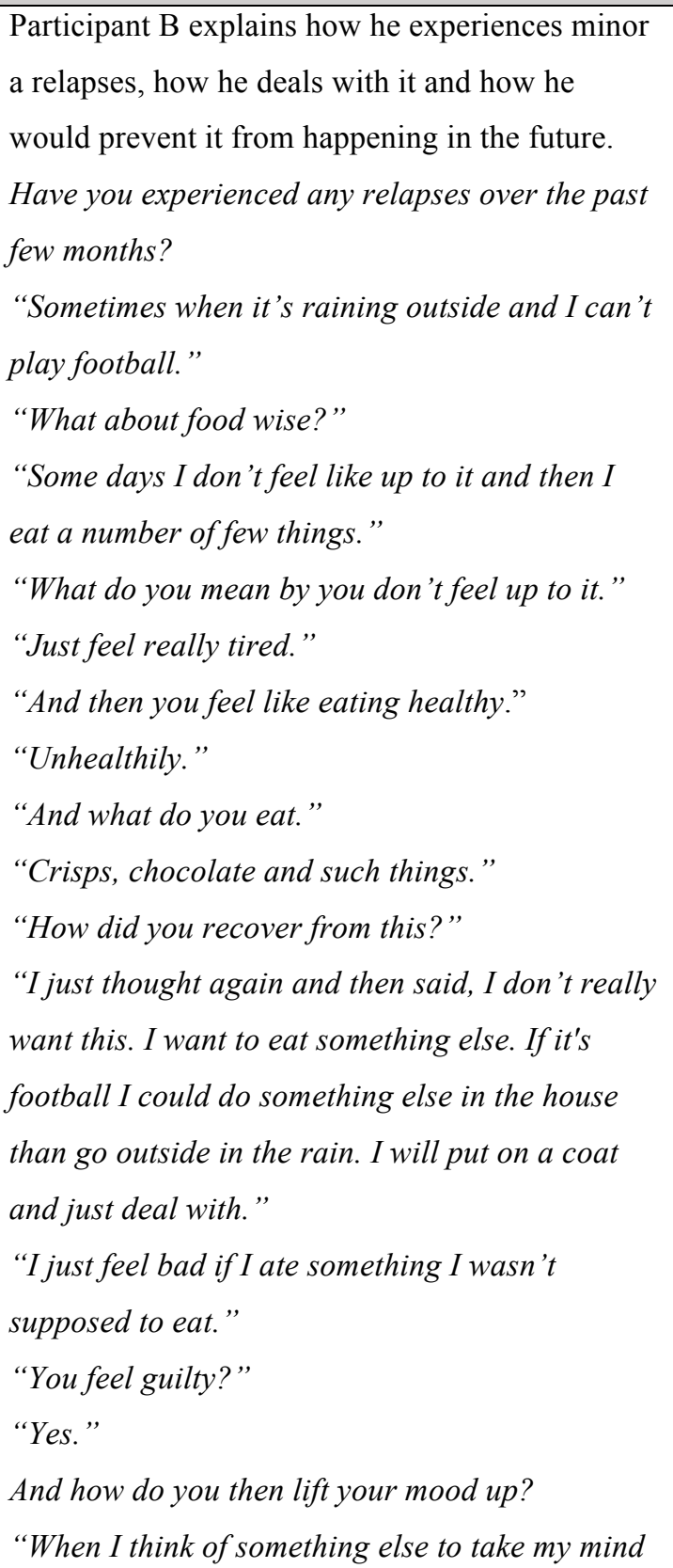 & $\begin{array}{l}\text { "Yes. It was particularly this week because we } \\
\text { both put on weight, where we went away and it } \\
\text { was a very nice week. So didn't do anything too } \\
\text { active and we were this and we found a very nice } \\
\text { restaurant as well and then we came home we both } \\
\text { went, whose going to lose the most?" } \\
\text { "We both had strength. Get up back and we both } \\
\text { did. He lost a couple and I did." }\end{array}$ \\
\hline
\end{tabular}




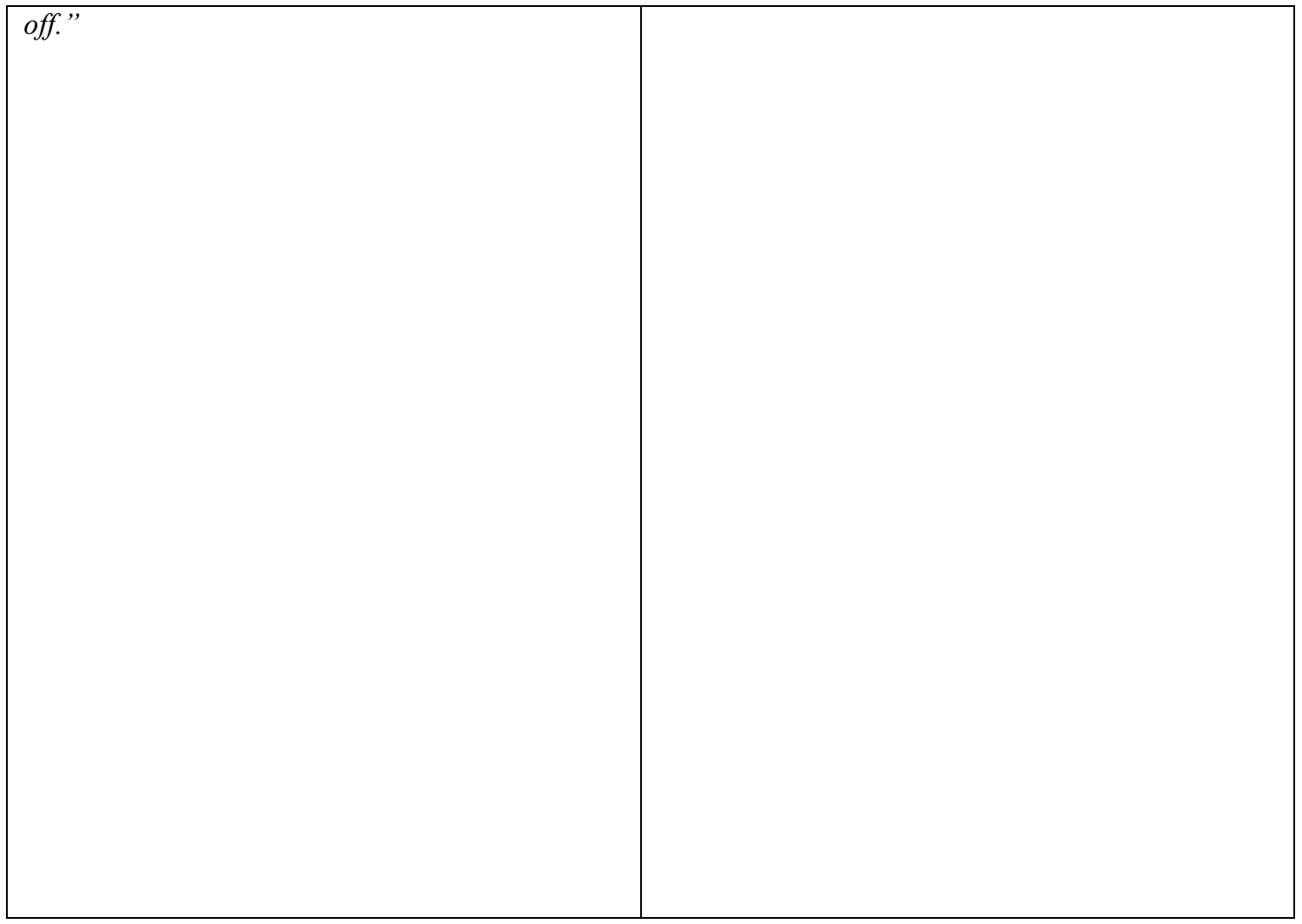

\begin{tabular}{|c|c|}
\hline \multicolumn{2}{|c|}{ Participant C } \\
\hline Child & Parent \\
\hline Effectiveness 6 months & Effectiveness 6 months \\
\hline $\begin{array}{l}\text { Preparation phase } \\
\text { Vulnerability, Outcome expectancy, perceived change at } \\
6 \text { months. }\end{array}$ & $\begin{array}{l}\text { Preparation phase } \\
\text { Vulnerability, Outcome expectancy, } \\
\text { perceived change at } 6 \text { months }\end{array}$ \\
\hline $\begin{array}{l}\text { Participant C understands the risks of type } 2 \text { diabetes } \\
\text { and know the importance of diet and exercise. But he } \\
\text { does not feel at risk himself. } \\
\text { "What do you think can reduce your risk?" } \\
\text { "Being healthy, eating healthy and just not eating junk } \\
\text { foods like all the time" } \\
\text { "Do you see yourself as being at risk?" } \\
\text { "Um... I did at one point but now I don't think I am at } \\
\text { risks anymore", } \\
\text { Semi structured interview at } 6 \text { months suggest that } \\
\text { participant C perceives the study to be effective as he } \\
\text { states that he has lost weight. } \\
\text { "Quite good, I've lost a lot of weight thanks to this [the } \\
\text { study]." This perception is not supported by the }\end{array}$ & $\begin{array}{l}\text { His mum believes he is at risk due to being } \\
\text { overweight and inactive. } \\
\text { "I don't think he is at risk because of my } \\
\text { husband but I do think he is at risk because } \\
\text { he is overweight and not active." } \\
\text { His mother believes taking part in the study } \\
\text { has had a positive effect on her sons diet } \\
\text { due to the changes that have been made as a } \\
\text { family but she thinks they are not following } \\
\text { the recipe book provided and is doubtful } \\
\text { that her son has made any changes himself } \\
\text { outside of the home. } \\
\text { "From the food point of view we are not } \\
\text { following what is in there [the cook book] at }\end{array}$ \\
\hline
\end{tabular}


measured outcomes of weight or BMI (table 1) and the children were not given a target to lose weight.

Participant $\mathrm{C}$ perceives his diet to have improved and believes he is more active. They have made some changes as a family and cook from the provided cookbook at least on occasion.

"I've cut down on all the junk food (burgers and pizza mainly) I used to eat, used to have them a lot but barely any now, cakes and that seeing that school makes vegetarian options it's easier to do it now." "Walk to school and back, and I go on my bike a lot."

"Yeah, we just had salmon for tea and that's from the cook book."

Are you able to perform the planned activity?

"Yes, sometimes I struggle when I have to do my school work" all. But we are changing what we are eating so we are hopefully having something from that but then he will go and have lunch, I don't know what he's had for lunch. From my point of view there has been a lot of positives.... He is eating differently.

"How has it influenced your son's attitude, skills, knowledge and understanding, behaviour?

"I don't know that it has."

"It's quite good because it does force you to start thinking no actually this is not good to carry on doing, because you do fall into a

habit. That's quite nice to see that but I wouldn't say that's been entirely successful."

His mother believes he is not very keen on performing physical activity. The only examples of physical activity participant $\mathrm{C}$ provided were walking to school or cycling. Yet, his mother explains that he has been on holiday and the friend he goes cycling with has broken his leg so he has not been cycling for a while.

"I think so, he is not that keen on doing it anyway. for example cycling. He goes cycling with his friend, unfortunately his friend broke his leg a week after we saw you, so he hasn't been out since. So, although Luke has other friends he could go cycling with, he will go down and see his mate because his mate has plaster on his leg and can't go cycling."

"I think in a theoretical way he does but I'm not sure that he applies that to his everyday life."

\section{Competency/Self-efficacy 6 months}

Competency/Self-efficacy 6 months

Participant $\mathrm{C}$ is confident in his ability to choose healthy food and be active.

"How competent do you feel in choosing healthy food
His mother also believes he is very able but questioned whether he performs the behaviours he talks about. 


\begin{tabular}{|c|c|}
\hline $\begin{array}{l}\text { and exercising?" } \\
\text { "Quite competent now" } \\
\text { "I've cut down on all the junk food (burgers and pizza } \\
\text { mainly) I used to eat, used to have them a lot but barely } \\
\text { any now, cakes and that seeing that school makes } \\
\text { vegetarian options it's easier to do it now" } \\
\text { "Walk to school and back, and I go on my bike a lot." } \\
\text { "Are you able to plan a healthy diet?" } \\
\text { "Yes - for breakfast I'll have Weetabix because there is } \\
\text { not a lot of sugar in it and wholegrain for lunch } \\
\text { probably hummus, for tea I'll have salmon again } \\
\text { because it was quite nice, veg? yeah carrots ." } \\
\text { "Are you able to plan activities for 6omin + every day? } \\
\text { E.g." } \\
\text { "Yes quite easily, this weekend I have been doing a lot } \\
\text { of cycling and exercise" }\end{array}$ & $\begin{array}{l}\text { "He is very competent but I'm not sure that } \\
\text { he appreciates why he needs to do it. I know } \\
\text { that he does need to do it", } \\
\text { "I think in a theoretical way he does but I'm } \\
\text { not sure that he applies that to his everyday } \\
\text { life." } \\
\text { "Is he able to plan a healthy diet?", } \\
\text { "I think if sat down and thought about it, } \\
\text { yes but I don't think he does. He is a } \\
\text { teenage boy I don't think he does." } \\
\text { "Is he able to plan activities for 6omin + } \\
\text { every day? E.g." } \\
\text { "He should be able to, but there is always } \\
\text { something else to do. So, I think that is } \\
\text { proving difficult." }\end{array}$ \\
\hline $\begin{array}{l}\text { Coping self-efficacy } \mathbf{1 2} \text { months } \\
\text { Participant C's most consistent source of activity is his } \\
\text { walk to school and back, however, he is now on holiday. } \\
\text { "Um.... If I were to go out um... as I'm not in school at } \\
\text { the moment I'm still determined to walk the same } \\
\text { amount as I would normally when I walk to school and } \\
\text { back." } \\
\text { He believes he can replace his school walk by running } \\
\text { around with his friend but does not plan specific } \\
\text { activities or monitor his activity time. } \\
\text { "Ok, so have you been doing that?" } \\
\text { "Um.. nearly all the time yes." } \\
\text { What have you done to replace the school walk? } \\
\text { "I have been going down, running around playing } \\
\text { games and stuff with my friends." }\end{array}$ & Copi \\
\hline Competency Self-efficacy 24 months & Competency Self-efficacy 24 months \\
\hline $\begin{array}{l}\text { He provides an example of a relapse and attributed it to } \\
\text { exams at school. Once the exams were done he was able } \\
\text { to recover. } \\
\text { "Have you experienced a relapse" } \\
\text { "A couple of times. I want to be doing exams without } \\
\text { stress so I will eat a bit more than I should." } \\
\text { "I was definitely lacking on exercise because I was } \\
\text { staying in and revising mainly. But now I have got the }\end{array}$ & $\begin{array}{l}\text { His mum believes he is susceptible to peer } \\
\text { pressure which may lead to relapses } \\
\text { "I think if he's with his mates and they are } \\
\text { all getting chips and that sort of thing, he } \\
\text { does find the peer pressure and he just goes } \\
\text { along with it. Not unwillingly as well so I } \\
\text { would say yes he's probably had some } \\
\text { relapses." }\end{array}$ \\
\hline
\end{tabular}




\begin{tabular}{|c|c|}
\hline $\begin{array}{l}\text { whole summer to continue exercising." } \\
\text { "I think I put on a little bit weight which was not good. I } \\
\text { was trying to get rid of that extra weight." } \\
\text { "Once exams were finished I just wanted to go out and } \\
\text { my friend has recently moved down in Mumbles. So we } \\
\text { can go down there all day quite recently."It doesn't } \\
\text { make me feel good that I've... but I just remember that I } \\
\text { can break the habit and continue doing what I normally } \\
\text { do.” } \\
\text { He is confident in his ability } \\
\text { "How do you feel about your own ability to continue } \\
\text { with this behaviour?” }\end{array}$ & $\begin{array}{l}\text { Autonomy } 6 \text { months } \\
\text { "I suppose so, I just go and buy food. They } \\
\text { don't get a huge amount of choice. Yes he } \\
\text { chooses what he will have." } \\
\text { "Is he/are you allowed to refuse unhealthy } \\
\text { food" } \\
\text { "I can honestly say that I have never known } \\
\text { him refusing food. I'd say he won't be } \\
\text { forced, put it that way." } \\
\text { "Is he able to make healthy snacks" } \\
\text { "Yes he is" } \\
\text { His mother believes he is influenced by } \\
\text { what other people do and what his friend } \\
\text { eat. She also gives him space to make his } \\
\text { own decision as she wants the initiative to } \\
\text { be healthy to come from him. } \\
\text { "I think if he has somebody with him that } \\
\text { says let's go have some sweets he'll go and } \\
\text { do it." } \\
\text { "I think he needs to do it, if someone is } \\
\text { doing everything for you, you don't take } \\
\text { responsibility for it so I haven't had row } \\
\text { with him about doing exercise or stop him } \\
\text { eating because he should be doing it himself } \\
\text { but whether that's parenting or it's me } \\
\text { know without me telling him." }\end{array}$ \\
\hline
\end{tabular}




\begin{tabular}{|c|c|}
\hline Autonomy 12 months & Autonomy 12 months \\
\hline $\begin{array}{l}\text { Participant C is believed he was responsible for his own } \\
\text { diet and physical activity choices. } \\
\text { "How much of your behaviour is down to you?" } \\
\text { "All of it, so } 100 \% \text { ?" } \\
\text { He is at secondary school where he chooses his lunch } \\
\text { from the canteen, his other meals are provided at home. } \\
\text { His exercise comes from his walk to school and } \\
\text { spending time with friends. } \\
\text { "Now I'm not in school there is now a less chance that I } \\
\text { will eat cakes because we don't have them at home so } \\
\text { it's easier to eat a lot more healthier at home than at } \\
\text { school, because in school they sell a lot of cakes." } \\
\text { "Walking past shops with money or seeing my friends } \\
\text { when I have money, seeing my friends buy sweets and } \\
\text { that it can make you want to buy things." } \\
\text { "Most of my friends eat unhealthy so it can be a bit of a } \\
\text { distraction but I try to ignore it and continue working." }\end{array}$ & $\begin{array}{l}\text { However, his mother believed he needs a lot } \\
\text { of persuasion from her. } \\
\text { "How much of his behaviour is down to } \\
\text { him?" } \\
\text { "I'd probably say he is } 35-40 \% \text { is him" } \\
\text { "Well I suppose I am pushing him to do } \\
\text { things so there is an extent that I'm doing it, } \\
\text { I don't know that the school is particularly" } \\
\text { "What percentage would you give to you" } \\
\text { "55\%" } \\
\text { As he is getting older he is spending more } \\
\text { time with friends independent of adults and } \\
\text { gaining independence over dietary and } \\
\text { activity choices. } \\
\text { "again, his friends are getting older and } \\
\text { they can all go and there are no parents } \\
\text { involved so its independence." }\end{array}$ \\
\hline Action planning-Goal setting 6 months & Action planning-Goal setting 6 months \\
\hline $\begin{array}{l}\text { Participant C believes being to busy can get in the way } \\
\text { of him setting goals and performing activity. It is very } \\
\text { clear that taking part in the program or being active was } \\
\text { very low priority at this point as he is too busy or } \\
\text { forgets. } \\
\text { What do you find difficult in goal setting? } \\
\text { "Not knowing if I have the time in the end, if I set goal } \\
\text { and I have to do other things that'll be hard to } \\
\text { overcome" } \\
\text { "What stops you achieving your goals?" } \\
\text { "Not having time and forgetting, because I don't have } \\
\text { the best memory." }\end{array}$ & $\begin{array}{l}\text { Participant C's mother does not believe that } \\
\text { he is planning his physical activity or } \\
\text { defining his goals. } \\
\text { "Is s/he able to plan activities for 6omin + } \\
\text { every day? E.g." } \\
\text { "He should be able to, but there is always } \\
\text { something else to do. So I think that is } \\
\text { proving difficult." }\end{array}$ \\
\hline $\begin{array}{l}\text { Action planning/Goal setting, } 12 \text { months } \\
\text { (also self-monitoring and contracting) }\end{array}$ & $\begin{array}{l}\text { Action planning/Goal setting, } 12 \text { months } \\
\text { (also self-monitoring and contracting) }\end{array}$ \\
\hline $\begin{array}{l}\text { Participant C remembers his behaviour change contract } \\
\text { but does not remember his goals } \\
\text { "Do you remember the healthy behaviour contract that } \\
\text { you signed?" } \\
\text { "Yes" } \\
\text { "Do you remember your goals that were on it?" }\end{array}$ & $\begin{array}{l}\text { His mother does not think he uses goal } \\
\text { setting } \\
\text { "Does he remind himself of his goals?" } \\
\text { "I doubt it." }\end{array}$ \\
\hline
\end{tabular}




\begin{tabular}{|c|c|}
\hline $\begin{array}{l}\text { "NO" } \\
\text { "Do you still set goals for yourself?" } \\
\text { "Um... not all the time but I do set goals" } \\
\text { Yeah, what's the last goal you set? } \\
\text { "Um... I can't remember um.... I really can't } \\
\text { remember" } \\
\text { "Um... I think the last one was have um ... a piece of } \\
\text { fruit each day for a week, } 2 \text { weeks and } 3 \text { weeks" } \\
\text { Participant C set himself this goal in the first few weeks } \\
\text { of the study which suggests he has not been using goal } \\
\text { setting for a long time. } \\
\text { "Um.. well I don't really use goals I just do activities } \\
\text { just to do something instead of sitting around mainly." } \\
\text { Participant C does not use the self-monitoring } \\
\text { techniques he learnt in the meeting but provides some } \\
\text { other examples. He states, "I can" or "I could" rather } \\
\text { than "I do", which suggest that these are not actually } \\
\text { time I try and beat even mine and do it again." } \\
\text { things he does but more hypothetical examples. } \\
\text { "I can check my weight once a month and then check } \\
\text { my weight the next month to see if I made any } \\
\text { progress." Participant C does not monitor how much }\end{array}$ & $\begin{array}{l}\text { "How do you think goal setting helped him } \\
\text { to change his behaviour", } \\
\text { "Again, I don't know because I don't know } \\
\text { how much of it is that he is doing", } \\
\text { "I don't know whether its helped him set } \\
\text { goals because I don't know if its necessarily } \\
\text { done that but it's certainly made him more } \\
\text { aware of things, so not sure." }\end{array}$ \\
\hline Action planning Goal setting 24 months & Action planning Goal setting 24 months \\
\hline $\begin{array}{l}\text { Participant C does not use any goal setting technique or } \\
\text { monitor his progress or behaviours } \\
\text { "Do you have goals that you are trying to achieve." } \\
\text { "Overall to be healthier and fitter." } \\
\text { "Any specific goals?" } \\
\text { "Not really just those two overall ones." } \\
\text { "During the study we talked about self-monitoring, do } \\
\text { you currently use any methods of monitoring your own } \\
\text { behaviours, goals or progress?" } \\
\text { "Not all the time because sometimes I will remind } \\
\text { myself of why I'm doing this." }\end{array}$ & $\begin{array}{l}\text { "Does he set himself goals?" } \\
\text { "I don't think so. No. Not that he's told me } \\
\text { about no. } \\
\text { "Does he monitor his own behaviour" "Not } \\
\text { that I'm aware of, I don't think he does } \\
\text { anything like that." }\end{array}$ \\
\hline Relatedness/social support 6 months & Relatedness/social support 6 months \\
\hline The exercise he undertakes is always related to spending & His mum also perceives his friends to offer \\
\hline
\end{tabular}


time with friends

"the rest of the week I pretty much go outside and play with my friends",

However, he also believes that he eats more healthily than his friends and that they are source of temptations by exposing him to unhealthy foods.

"I feel I eat better than them [his friends] now than I did before."

"Yes pretty much- quite likely to maintain - maybe if I go with friends to town they wanna go to a shop and its full of sweets might be a bit hard then."

"If we are cycling then we go to one of their houses and they offer sugary drinks."

He also gets support from his family who have all made changes to adjust their diet as a family.

"Since this is happened [the program] they've changed as well, trying to eat healthier."

"A lot of support from my friends and family. My dad always encourages me after I have come back from our meeting or before I go to do an activity."

"Mum is always there when I go to the meetings and she always reminds me to do the activities." him temptations.

"I think it's mixed with the exercise, some of them [friends] are more active than others, quite a few of them are computer enthusiasts so yeah that's one of the things he would like to do. With food I'm not sure really because it does seem that like his friend who has broken his leg, always wanting to eat sweets, always asks do you have money, do you want to go to the shops? Buy sweets? So quite a bit of that going on I think. And the school food they always have cakes and sweets as an option with their meal."

His mother describes herself and his father as "not active" so they are not role models in this sense and do not model good physical activity behaviours at home.

"My husband and I are pretty much not that active so we are not that fit so I'm afraid Luke takes after both of us."

His mother perceives there to be a problem with his lack of physical activity to the extent that they have discussed quitting the study.

"I said if you are not doing the exercises do you want to carry on doing this [the study] and he does want to carry on doing it, so I'm not sure how much he appreciates it but I know he wants to carry on doing it." She describes their ongoing discussions about doing physical activity as a battle and believes she is constantly nagging him. "I'm very weary of it becoming a battleground and me making him not wanting to do it at all, so he is a bit better with eating and he certainly doesn 't kick off about it at all from that point of view. It's more the exercise that's a problem." "trying to get him to do more exercise is 


\begin{tabular}{|c|c|}
\hline & $\begin{array}{l}\text { difficult, so it's good but it's challenging" } \\
\text { "It's making him think about it but it's a } \\
\text { battle I think. Battle with me yeah.", }\end{array}$ \\
\hline Relatedness/Social support 12 months & Relatedness/Social support 12 months \\
\hline $\begin{array}{l}\text { His exercise comes from his walk to school and } \\
\text { spending time with friends. The main temptations he } \\
\text { describes are also related to choices at school or his } \\
\text { friends eating sweets, suggesting that school is not } \\
\text { always a supportive environment and his friends not } \\
\text { always a positive influence. } \\
\text { "Now I'm not in school there is now a less chance that I } \\
\text { will eat cakes because we don't have them at home so } \\
\text { it's easier to eat a lot more healthier at home than at } \\
\text { school, because in school they sell a lot of cakes." } \\
\text { "Walking past shops with money or seeing my friends } \\
\text { when I have money, seeing my friends buy sweets and } \\
\text { that it can make you want to buy things." } \\
\text { "Most of my friends eat unhealthy so it can be a bit of a } \\
\text { distraction but I try to ignore it and continue working." } \\
\text { Participant C believes his home environment is } \\
\text { supportive of healthy eating } \\
\text { "at home so it's easier to eat a lot healthier at home } \\
\text { and that his family offer him encouragement. } \\
\text { reminding me that I can do it" }\end{array}$ & 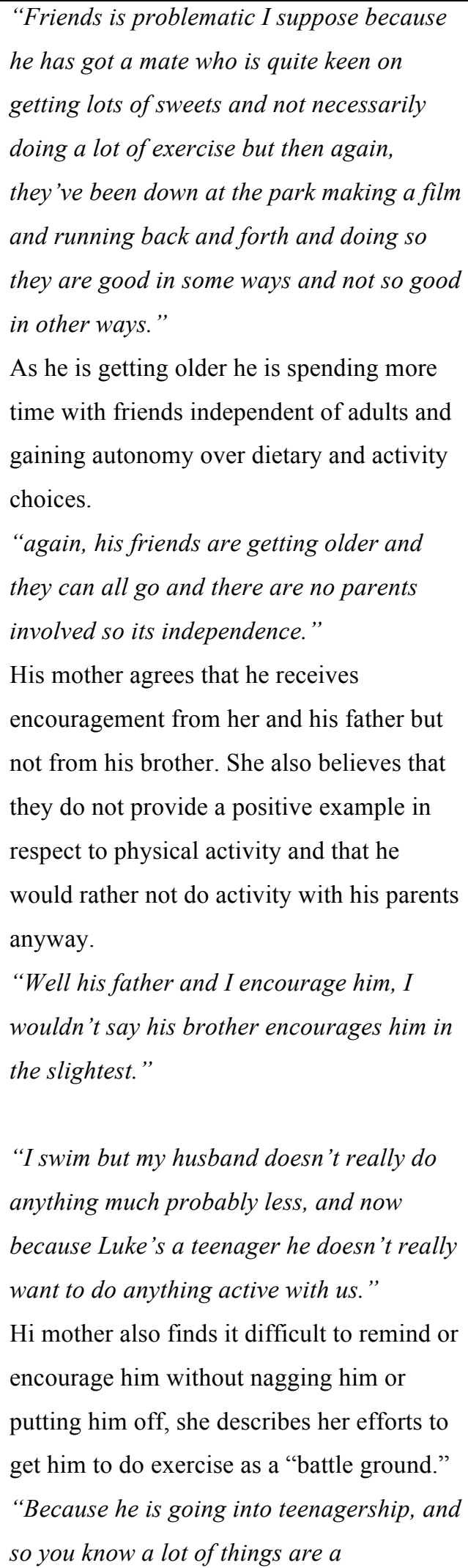 \\
\hline
\end{tabular}




\begin{tabular}{|c|c|}
\hline & $\begin{array}{l}\text { battleground" } \\
\text { "Me nagging him doesn't really help but } \\
\text { having better food around I suppose does } \\
\text { "my worry is that if I go on about it its } \\
\text { nagging which doesn't work." } \\
\text { "he is also entering cheerful teenager } \\
\text { territory so anything you say he will do the } \\
\text { opposite off." } \\
\text { "It's difficult to have a sensible } \\
\text { conversation because it's very 'I didn't ask } \\
\text { to be born' and thinking of this sort of } \\
\text { things.", }\end{array}$ \\
\hline Maintenance 12 months & Maintenance 12 months \\
\hline $\begin{array}{l}\text { Participant C believed that he has maintained some } \\
\text { behaviours, he is more active than he used to be and } \\
\text { feels fitter. } \\
\text { "I read labels if I thinks it's a bit too sugary or high in } \\
\text { fat so I won't eat it or drink it } \\
\text { Um... eat a lot more healthier now than I did a year } \\
\text { ago." } \\
\text { "I do a lot more exercise than I did a year ago, I can do } \\
\text { more things for longer that can keep me active than I } \\
\text { was a year ago." } \\
\text { "Um... I run } 400 \text { meters without stopping in a good } \\
\text { amount of time } \\
\text { But last year I would been able to do that very well." } \\
\text { "Yes, sometimes I have gone down when I don't feel } \\
\text { good or when I'm stressed out but I am confident I can } \\
\text { do it all the time." }\end{array}$ & $\begin{array}{l}\text { It is very difficult to determine whether } \\
\text { participant C has made any changes to his } \\
\text { behaviour in order to maintain them. } \\
\text { His mother believes he has not taken his } \\
\text { bike out for a long time, and walking to } \\
\text { school is something he already did before } \\
\text { the study. } \\
\text { [Cycling]"And even know his friend is back } \\
\text { cycling but he isn't so we don't know, we } \\
\text { tried suggesting it a couple of times but it's } \\
\text { sort of like yeah ok and he has taken it out } \\
\text { but then its, so no I think the cycling is sort } \\
\text { of fallen away a bit." } \\
\text { "Has Luke maintained his behaviour } \\
\text { change." } \\
\text { "Walking to and from school he has always } \\
\text { done anyway but he doesn't mind doing it } \\
\text { so that's good, food he is suggestable so I } \\
\text { think if you, you know he has started to take } \\
\text { his sandwiches for lunch and that's a lot } \\
\text { healthier and that's only because the exams } \\
\text { where on but he was still quite amenable to } \\
\text { that," }\end{array}$ \\
\hline Maintenance 24 months & Maintenance 24 months \\
\hline
\end{tabular}




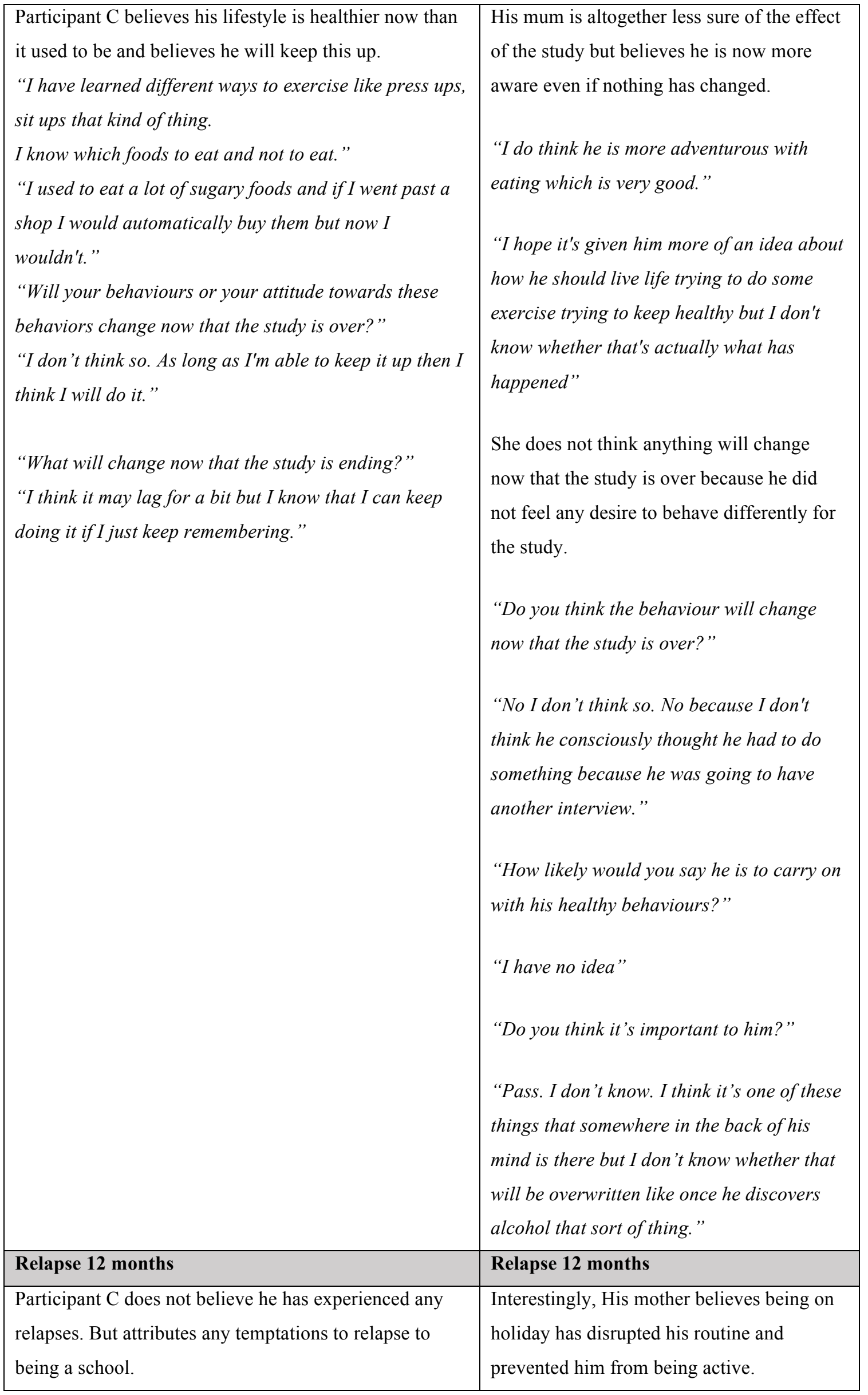




\begin{tabular}{|c|c|}
\hline $\begin{array}{l}\text { "Have you experienced any relapse at all?" } \\
\text { "No, since the start of summer holidays no, maybe } \\
\text { sometimes in school when I'm feeling down but apart } \\
\text { from that no" } \\
\text { "If I failed a test then I would feel down because I } \\
\text { would think I would be able to do that the I would eat } \\
\text { something sugary just to make myself feel better". } \\
\text { "Walking past shops with money or seeing my friends } \\
\text { when I have money, seeing my friends buy sweets and } \\
\text { that it can make you want to buy things." } \\
\text { Visualization and self-talk } \\
\text { Participant C does not use the visualization or self-talk } \\
\text { we discussed in group meetings. } \\
\text { "Do you use visualization techniques?" } \\
\text { "No, not really" } \\
\text { "Ok, do you ever use self-talk?" } \\
\text { "I did for a little bit but I find it wasn't really helping } \\
\text { me get anywhere so I decided to do something else." }\end{array}$ & $\begin{array}{l}\text { Being on holiday doesn't help, not having } \\
\text { any set routines, so given the choice he is } \\
\text { happy to stay in bed all day or sit and watch } \\
\text { the telly all day he doesn't got the same } \\
\text { thing except with school you've got to get } \\
\text { dressed you got to go for a walk whereas ..” } \\
\text { " so his friend was away for the first week } \\
\text { of the holidays so I was trying to you know } \\
\text { jimmy him out of the house so yes that } \\
\text { makes it more difficult”. }\end{array}$ \\
\hline Relapse 24 months & Relapse 24 months \\
\hline $\begin{array}{l}\text { Have you experienced any relapses over the past few } \\
\text { months? } \\
\text { "A couple of times. I want to be doing exams without } \\
\text { stress so I will eat a bit more than I should." } \\
\text { What about your exercise? } \\
\text { "I was definitely lacking on exercise because I was } \\
\text { staying in and revising mainly. But now I have got the } \\
\text { whole summer to continue exercising." } \\
\text { "How are you affected by a relapse?", } \\
\text { "I think I put on a little bit weight which was not good. I } \\
\text { was trying to get rid of that extra weight." } \\
\text { "How does it affect you psychologically?" } \\
\text { "It doesn't make me feel good that I've... but I just } \\
\text { remember that can break the habit and continue doing } \\
\text { what I normally do." }\end{array}$ & $\begin{array}{l}\text { "Has he experienced any relapses in the } \\
\text { past months or year?" } \\
\text { "I think if he's with his mates and they are } \\
\text { all getting chips and that sort of thing, he } \\
\text { does find the peer pressure and he just goes } \\
\text { along with it. Not unwillingly as well so I } \\
\text { would say yes he's probably had some } \\
\text { relapses. But certainly as I say he is far } \\
\text { more adventurous and happy to be } \\
\text { vegetarian." } \\
\text { "How did he recover from the relapse?" } \\
\text { "He ran out of money and had to eat at } \\
\text { home." } \\
\text { "How is he affected by a relapse?" } \\
\text { "Not at all". }\end{array}$ \\
\hline
\end{tabular}




\begin{tabular}{|c|c|}
\hline \multicolumn{2}{|c|}{ Participant D } \\
\hline Child & \\
\hline $\begin{array}{l}\text { Preparation phase } \\
\text { Vulnerability, Outcome expectancy, perceived } \\
\text { change at } 6 \text { months. }\end{array}$ & $\begin{array}{l}\text { Preparation phase } \\
\text { Vulnerability, Outcome expectancy, } \\
\text { perceived change at } 6 \text { months }\end{array}$ \\
\hline $\begin{array}{l}\text { It is unclear whether participant D perceives herself } \\
\text { to be at risk or vulnerable to type } 2 \text { diabetes, } \\
\text { "Do you think of yourself of being at risk?" } \\
\text { "Umm... when I found out from the meetings form } \\
\text { the graph you showed me that I was classed as } \\
\text { overweight but not obese I was very... it me like I } \\
\text { want to improve on my exercise so I can move from } \\
\text { obese down to a normal weight then down to a } \\
\text { weight that I want to be at." } \\
\text { Although she is motivated by her weight she does } \\
\text { not mention vulnerability to illness. She understands } \\
\text { that a poor diet is related to diabetes but does not } \\
\text { connect excess body weight to diabetes risk. }\end{array}$ & $\begin{array}{l}\text { Her mum is very clear that she is at risk and } \\
\text { her expected outcome of the behaviour } \\
\text { change is to reduce risk. } \\
\text { "Do you think she is at risk?" } \\
\text { "Yeah - the blood test results frightened } \\
\text { me" } \\
\text { What can reduce their risk? } \\
\text { "Eat healthily, exercise, keep that going } \\
\text { and hope for the best." } \\
\text { Her mother has found the study informative } \\
\text { and believes they have made changes to } \\
\text { their diet and exercise. } \\
\text { "I'm more aware of what we are eating, } \\
\text { what I'm eating, what I'm buying what I'm } \\
\text { bringing to the house, more aware for } \\
\text { Catlin of getting her to do exercise and } \\
\text { make sure it's regular and effective and I } \\
\text { suppose it's helping me to improve my } \\
\text { understanding of why we need to do what } \\
\text { we doing and doing exercises, why the } \\
\text { change in eating habits is needed, it's been } \\
\text { great }\end{array}$ \\
\hline $\begin{array}{l}\text { "I could like focus on the healthy things and the } \\
\text { good things and not think about the bad things and } \\
\text { bad food." } \\
\text { "What do you think the outcome of keeping healthy } \\
\text { behaviour is?" } \\
\text { "I think the outcome like doing all the work would } \\
\text { help me get my goal of being slimmer more fitter so } \\
\text { it will take time but eventually I would get there." } \\
\text { Participant D believes she has learnt how to choose } \\
\text { healthy foods and the importance of regular } \\
\text { exercise. As a result, she has lost weight and is } \\
\text { pleased with her progress. }\end{array}$ & $\begin{array}{l}\text { You have seen our little board, it's changed } \\
\text { a little bit. We are now doing something } 6 \\
\text { days a week. Every now and then she does } \\
\text { need a little shove but when we get going } \\
\text { she is fine, } \\
\text { "that it's becoming just part of the day for } \\
\text { her, and she misses it if we don't do it, } \\
\text { automatically she says we got this on } \\
\text { tonight and she is looking forward to that, } \\
\text { we have started a Zumba class now, first } \\
\text { one was last week and now she cannot wait }\end{array}$ \\
\hline
\end{tabular}




\begin{tabular}{|c|c|}
\hline $\begin{array}{l}\text { "I've learnt how bad it is for you to eat certain food } \\
\text { because of fat and calories in it and to check the } \\
\text { label." } \\
\text { "I've learnt that it is more important to eat } \\
\text { wholemeal bread than white bread because white } \\
\text { bread is not exactly the best bread to eat." } \\
\text { Being active and exercising is healthy when you } \\
\text { burn more calorie, stuff like just walking or running } \\
\text { or something." } \\
\text { "I've lost a little bit of weight, feeling happy, I've } \\
\text { achieved the fact that I can fit into the clothes that I } \\
\text { couldn't before like jeans, and stuff so I'm just } \\
\text { happy about that." } \\
\text { She describes the changes she has made at home } \\
\text { with her mother. } \\
\text { "it must be a massive change for her as well, } \\
\text { because we have changed everything in our } \\
\text { into a regular exercise routine planning out } 60 \\
\text { micer." } \\
\text { wet into better routine of things. Me and my mum } \\
\text { have made a little routine for activity on the diary } \\
\text { planner thing and }\end{array}$ & for this Thursday to come back round." \\
\hline Competency/Self-efficacy 6 months & Competency/Self-efficacy 6 months \\
\hline $\begin{array}{l}\text { Participant D shows she can choose the right food } \\
\text { and identify points of decision to choose healthy } \\
\text { options and overcome the temptation to eat } \\
\text { unhealthy food } \\
\text { "I've learnt that it is more important to eat } \\
\text { wholemeal bread than white bread because white } \\
\text { bread is not exactly the best bread to eat," } \\
\text { "I've overcome the challenges to just go up to the } \\
\text { shop and feel like buying a chocolate bar or a } \\
\text { packet of crisps. My friend said to me oh ..... let's } \\
\text { go up the shop but I said no and I sat on my door }\end{array}$ & $\begin{array}{l}\text { Her mum suggests that she struggles in } \\
\text { certain areas and needs frequent prompts } \\
\text { "She struggles in some situations, she just } \\
\text { tries her best and she finds it harder in } \\
\text { school, she definitely struggles in school } \\
\text { with what she describes as " they have the } \\
\text { nice options before they have the healthy } \\
\text { options". } \\
\text { Participant D and her mum plan their } \\
\text { activity and diet together but her mum does } \\
\text { not think she could do this herself }\end{array}$ \\
\hline
\end{tabular}


step and waited for her to go up to the shop.

"I am able to choose healthy food options when they are available, but every now and then I get just one unhealthy thing just for a little sugar boost, in school I choose these salad baguette, spicy chicken salad baguette, stuff like that."

However, on two occasions she states that she needs to believe in herself more suggesting she is not always confident in her own ability.

"What would help me maintain my healthy behaviour is the fact that I know that I can do it, I just have to believe in myself more, boost my confidence to give up unhealthy food and carry on." "I feel alright with the exercise and that and the healthy food, I feel like I am able to do it [exercise] I just have to like believe in myself more."

She later says that she would not be able to manage on her own

"I don't find it difficult when I got my mum to motivate me and stuff, she is basically like a saviour because if I did it on my own I wouldn't have a clue what to do"
"No she has other difficulties so she can't

do it, she'll help, when I'm cooking

something or planning something but she can't foreplan it herself."

"Is she able to plan activities for 6omin+ every day"

"With some encouragement, yeah as I said she is getting there, as you have seen our little board, it's changed a little bit. We are now doing something 6 days a week" "Is she able to choose healthy foods?" “Mostly, yeah I wouldn't say she's got a full knowledge but she goes for more healthier options in all fairness to her."

\section{Coping self-efficacy 12 months}

She describes how she recovers from a relapse by remembering her medals and the progress she has made. She also mentions her mum's role in helping her cope with a relapse and picking her up.

"Like if something starts going downhill it will probably make me less and less motivated because I will start feeling bad about myself if I forget to do a certain thing so my mum would remind me I'm not doing a bad job and I just need to kick start the routine back up and just remember the good progress I made."

"Because of the medals and stuff that I have won from the races and I would think about the stuff I have achieved over the past year and thing about

\section{Coping self-efficacy 12 months}

Her mum does not believe she learns from her relapse but just looks ahead.

"I don't think she learned anything to be honest because that's not Caitlin. With other is at doesn't come to the equation, it's happened, it's gone and that's that. She only looks forward and what we do now and tomorrow and not what happened yesterday."

Taking part in organised run was great for her self-belief.

"I don't think she had that self-belief in herself specially from doing the runs and you know, we've done the rainbow run and 


\begin{tabular}{|c|c|}
\hline $\begin{array}{l}\text { like how have I achieved them and if I continue } \\
\text { doing the stuff I am and it will help me to be } \\
\text { motivated for the future" } \\
\text { "My mum would make sure I'm happy and when I'm } \\
\text { not in a bad mood she would make sure I got to the } \\
\text { gym. She pretty much helps me forget about the bad } \\
\text { stuff and makes me remember the project and the } \\
\text { efforts I made in to that." } \\
\text { As a result, she is confident in her ability to } \\
\text { overcome another relapse } \\
\text { "If I were to have another relapse I definitely think I } \\
\text { could overcome it from the previous relapse that I } \\
\text { have had and I know how to reverse relapse." }\end{array}$ & $\begin{array}{l}\text { the muddy run, she knows she can do them } \\
\text { and she has achieved that whereas she } \\
\text { wasn't able to do that before and as I said } \\
\text { that self-belief in herself has given her a } \\
\text { right proper boost." }\end{array}$ \\
\hline Competency Self-efficacy 24 months & Competency Self-efficacy 24 months \\
\hline $\begin{array}{l}\text { Both participant D and her mum are aware that they } \\
\text { have experienced a relapse. } \\
\text { "I'm still struggling on the path where my friends } \\
\text { are eating junk and stuff in school. I have a bit of a } \\
\text { relapse in a eating a bit of junk. But recently over } \\
\text { the past few weeks haven't really ate junk. I have } \\
\text { gotten better." } \\
\text { Their action plans to prevent relapse are to make } \\
\text { packed lunches instead of eating in the canteen and } \\
\text { to not have money at school. } \\
\text { "My mom started giving me packed lunches with } \\
\text { salads wraps and apples and water." } \\
\text { "I'd probably stay on practice then when my mom } \\
\text { doesn't give me money then I don't feel the need to } \\
\text { grab some cake and stuff." }\end{array}$ & $\begin{array}{l}\text { Her mum believes she can get back on } \\
\text { track because she knows she has done it } \\
\text { before } \\
\text { "She has seen previously how good she has } \\
\text { felt, how good the changes have been and } \\
\text { she wants that again. So it's that drive now. } \\
\text { The last couple months haven't been good } \\
\text { but she wants to get to that point again. She } \\
\text { is definitely enthusiastic for it which is } \\
\text { always half the battle." } \\
\text { Her mum believes she is fairly confident in } \\
\text { her own ability but says she need to push to } \\
\text { keep her on track. } \\
\text { "It's not a matter of not knowing. She } \\
\text { knows- She does need that push and to } \\
\text { show that's why you want to choose } \\
\text { something else. But } 9 \text { times out of } 10 \text { she } \\
\text { will pick the better direction. I suppose she } \\
\text { is fairly confident but she does need that, } \\
\text { "is that really what you want?" }\end{array}$ \\
\hline Autonomy 6 months & Autonomy 6 months \\
\hline $\begin{array}{l}\text { Participant D is at secondary school where she } \\
\text { chooses her lunch from the canteen. } \\
\text { "I am able to choose healthy food options when they } \\
\text { are available, } \\
\text { "in school I choose these salad baguette, spicy }\end{array}$ & $\begin{array}{l}\text { "I give her a bottle of water and two pieces } \\
\text { of fruit everyday and she has money to } \\
\text { choose the main meal, because she sits with } \\
\text { her friends then, that's a bit of a 50/50" } \\
\text { At home her mum prepares meals, they go }\end{array}$ \\
\hline
\end{tabular}


chicken salad baguette, stuff like that."

She can make herself food in the house

"I am able to make a healthy snack or just food for myself in the house because we got equipment and the right food to make it. We haven't done it yet but we will be making Humus soon, with the little food blender, we make like pasta salads and stuff like that so.."

All of participant D's exercises are performed together with her mum, when asked if she is able to perform the activities she plans, she replies with "we".

"Most of the time we are but like when one of us is ill or something we just move around to a different day - to the best of my ability yeah"

“[Getting 60 minutes of PA] Yeah I'm able to do that because, me and my mum go to the gym and every Thursday we go to Zumba now so it's just easy"

The above statement suggest that she is reliant on her mum and would not exercise of her own volition if her mum was ill. shopping together and she can have some input over what they buy.

"I'll buy what she chooses if I agree they are healthy, she tries to sneak in the little.." "But she makes her own choices when we are out and about, she usually helps if I'm making something in here, if we are out and about then she tries her best to go for healthier options."

"Yeah, she does yeah. She will finish the salad and that from the fridge and I'll go to look for a meal and it's all gone,

It is clear that her mum is the driving force behind her exercise and she is relient on it to motivate her.

"I'm always pushing her to go here and there and anywhere, we do as much as we can and we go down the beach she uses all the exercise bits down the front and she seems quite happy with that so,"

"There are days that we need to give her a good shove to do the activity we have got planned but once she gets going, she goes to the other end of the scale then, she goes super excited about it and motivated."

Autonomy 12 months

Autonomy 12 months

Participant D does everything with close watch from her mum. They exercise together, take part in fun runs and her mum is always watchful of the food she eats.

"Um... possibly my mum, she helps me choose the right food to eat"
She needs a bit of pushing. 50\% is me and $10 \%$ is the environment and friends."

“As long as I push it, she'll be fine. She doesn't have a group of friends as such, she only has one or two friends and none of them are exercise driven. So it's only me that keeps her in this zone."

Do you think she needs that external motivation that comes from you?

"Yeah-definitely so, She needs to be monitored to keep on track but I think she wants to do it" 


\begin{tabular}{|c|c|}
\hline & $\begin{array}{l}\text { Participant D still relies heavily on her } \\
\text { mum to motivate her, to exercise with her } \\
\text { and to prevent her from overeating. } \\
\text { "Maybe she could start learning to start } \\
\text { doing things on her own a bit more. She } \\
\text { knows I can't run but if we walk in the dark } \\
\text { down the beach she'll run off ahead of us } \\
\text { and wait for us at some point and turn } \\
\text { around and run back. So we are there but } \\
\text { she takes on herself to do the running. Its } \\
\text { starting, it's just going to need a bit more } \\
\text { work to keep it going" }\end{array}$ \\
\hline Action planning-Goal setting 6 months & Action planning-Goal setting 6 months \\
\hline $\begin{array}{l}\text { [About setting goals] } \\
\text { "It does help me because it makes me feel better } \\
\text { after we've done all the things in the planner and I } \\
\text { can literally feel the difference after I've done all } \\
\text { the things that I've planned makes me happy" } \\
\text { "Me and my mum have made a little routine for } \\
\text { activity on the diary planner thing and we've gotten } \\
\text { into a routine of we know what we got to be doing } \\
\text { exercise wise." } \\
\text { Participant D demonstrates that she remembers and } \\
\text { uses the action planning we discussed in the } \\
\text { meeting. She identifies the barrier (going to the } \\
\text { shop) and comes up with an action to perform in this } \\
\text { scenario. } \\
\text { I've overcome the challenges to just go up to the } \\
\text { shop and feel like buying a chocolate bar or a } \\
\text { packet of crisps. My friend said to me oh Caitlin } \\
\text { let's go up the shop but I said no and I sat on my } \\
\text { door step and waited for her to go up to the shop. }\end{array}$ & $\begin{array}{l}\text { Participant D and her mother plan out the } \\
\text { weeks exercise on a black board in the } \\
\text { living room and use the daily planner } \\
\text { provided in the meetings. They set } \\
\text { themselves targets and have got a reward } \\
\text { system in place } \\
\text { "Our reward system is that we go to the } \\
\text { LC2, or just go out for the day and that'll } \\
\text { be her decision whatever we do. Once } \\
\text { she's done the exercises for a fortnight or } \\
\text { such that, she gets to choose what we do, } \\
\text { she thinks she is the boss then, she enjoys } \\
\text { it, she does see it as a reward and we } \\
\text { enforce it that it's the reward for her efforts } \\
\text { and her achievements and she is } \\
\text { delighted." }\end{array}$ \\
\hline $\begin{array}{l}\text { Action planning/Goal setting, } 12 \text { months } \\
\text { (also self-monitoring and contracting) }\end{array}$ & $\begin{array}{l}\text { Action planning/Goal setting, } 12 \text { months } \\
\text { (also self-monitoring and contracting) }\end{array}$ \\
\hline $\begin{array}{l}\text { Participant D remembers the behaviour change } \\
\text { contract and some her goals she set. } \\
\text { "Do you remember you healthy behaviour contract } \\
\text { that we signed?" } \\
\text { "Yeah" } \\
\text { "What does it say in it?" }\end{array}$ & $\begin{array}{l}\text { "She mentions the goals. She wants to be } \\
\text { fitter and healthier. She has this vision now } \\
\text { in her own head that she is } 6 \text { foot and a } \\
\text { model and she 'll be in a bikini on the } \\
\text { beach." } \\
\text { Participant D, demonstrated how she }\end{array}$ \\
\hline
\end{tabular}


"It says if I do well I get Lc2 tokens haha"

Participant $\mathrm{D}$ is motivated by her body image, she

has a long-term goal related to her appearance and monitors her progress by looking in the mirror.

"My goals were to achieve a super model body and to look good in a bikini",

"By looking at myself in the mirror and realising the shape that I am is all because of the working out I done"

She also remembers her more serious goals to no longer be classified as "overweight"

"Umm... when I found out from the meetings form the graph you showed me that I was classed as overweight but not obese I was very... it me like I want to improve on my exercise so I can move from obese down to a normal weight then down to a weight that I want to be at."

Although she is no longer writing down goals on her planner she (with help from her mum) set short term which adhere to the SMART principles by signing up for running events and training for these events. "I set a goal on the rainbow run that I did...

Working up to it was a goal.”

"Yeah, the Christmas run with the exercise I done it has made me want to set a goal each time I go there because the distance was further was further from $1 \mathrm{k}$ to $5 \mathrm{k}$ because I managed to run it, it makes me want to set different distances every time I do a race."

Participant D does not monitor her behaviours, she believes she can remember her old dietary behaviours and doesn't want to go back to that. "Because I remember the stuff I used to eat like ordering chips form the chippy from up the road or ordering a pizza, I would be remembering what I used to order those and remind myself what I been eating now like pasta with sweetcorn and tuna." She does monitor her progress with help from her grandparents

"I'm able to monitor my own progress, yeah I think overcomes barriers to her achieving her goals with action planning. She acknowledges that she will give into temptations so avoids the situations by planning.

"she's asking me to make her something healthy for lunch because she is fed up now with the food choices in the canteen and having all the cookies and the biscuits and one tiny bowl of fruit or something at the back of the till, so she's like mum I can't do this, I'm gonna eat them if I keep going in there. So she is getting me to make lunch for her for school now. She can't be bothered with the battle anymore, you know it's working. That's her strategy. It's keep away from the temptations, if she sees cookies she won't stop with one she will have them all."

[Monitoring behaviour]

"No she wouldn't bother"

"We monitor her weight, we monitor her height, sometimes she gets down but she doesn't realise she is growing upwards so she is getting heavier." 


\begin{tabular}{|c|c|}
\hline $\begin{array}{l}\text { I am because umm... my grandparents when I } \\
\text { started my little weight loss measuring thing [BMI } \\
\text { calculator] when they have this box and inside the } \\
\text { box they have this little measuring thing where they } \\
\text { write down my record every week so that gave me } \\
\text { an idea of having my own little diary so whenever I } \\
\text { go up my grandparents house I wrote down my rates } \\
\text { in the little diary which reminds me of the progress } \\
\text { that I have made." }\end{array}$ & \\
\hline Action planning Goal setting 24 months & Action planning Goal setting 24 months \\
\hline $\begin{array}{l}\text { Participant D monitors her progress by measuring } \\
\text { west circumference but does not monitor her } \\
\text { behaviour. } \\
\text { I have started to measure my waist every few days } \\
\text { and write it down in a book I have to keep track. } \\
\text { "What about your behaviour? So what you eat and } \\
\text { what you do and how much you sit down, how much } \\
\text { you are in front of the TV or screen. These are all } \\
\text { behaviours. Do you monitor that?? } \\
\text { "No. Not really." }\end{array}$ & $\begin{array}{l}\text { Participant D and her mother do not set } \\
\text { goals anymore but have got an exercise } \\
\text { routine. } \\
\text { "I think she just has a mind-set. We don't } \\
\text { really have goals anymore. It's just she } \\
\text { knows what she is going to do. She knows } \\
\text { when she is not trying as hard as she could. } \\
\text { She knows when she is trying hard. She can } \\
\text { see the differences in herself and feel the } \\
\text { differences." } \\
\text { "it's just becoming part of our weekly } \\
\text { routine now. We don't really set goals as } \\
\text { such." }\end{array}$ \\
\hline Relatedness/social support 6 months & Relatedness/social support 6 months \\
\hline $\begin{array}{l}\text { Participant D mentions her friends as a source of } \\
\text { temptation to eat unhealthy foods } \\
\text { I got this friend across the road which is like all she } \\
\text { does is spending money on cans of cola, chocolates } \\
\text { and crisps and stuff," } \\
\text { "My friend said to me oh .. let's go up the shop but I } \\
\text { said no.." } \\
\text { She is very close with her mum and receives support } \\
\text { and encouragement, her mum performs physical } \\
\text { activity with her modelling healthy behaviour. } \\
\text { "I relate to my mum because she is supporting me } \\
\text { and she is doing this with me", } \\
\text { "Yeah she is really supportive, she boosts me when } \\
\text { I'm having a down day, I'm like no I'm not doing it } \\
\text { and when I do I just feel better and wanna continue } \\
\text { doing it", }\end{array}$ & $\begin{array}{l}\text { Her mum also mentions her friends as a } \\
\text { source of temptation. } \\
\text { "She struggles a bit I think, she's got a } \\
\text { friend across the road and they go up the } \\
\text { corner shop quite often, because her } \\
\text { friends likes to snack and munch on sweets } \\
\text { and stuff and she finds that difficult to be } \\
\text { put in that situation.." } \\
\text { "I motivate her, I keep her on track, I pick } \\
\text { up the little sneaky treats (it's fruit mum) } \\
\text { no it's not Caitlin. I just keep going } \\
\text { whatever, if she wants to change something } \\
\text { we change it, if she is happy we carry on } \\
\text { with what we got. Keep on going, keep } \\
\text { pushing her, keep encouraging her for as } \\
\text { long as we can." }\end{array}$ \\
\hline
\end{tabular}


Her grandparent also offer positive support, accompanying her to meetings when her mum is at work. "In their [grandparents] food they do vegetables, my nan makes her own bread, she does cranberry and rice or something bread and she makes us these really healthy smoothies, banana, mixed berries and water or type of juice -

School in not a supportive environment for good food choices

"When I go to school when in the break time you go down this little corridor thing and when you go down there right in front of you is a tray of cakes and stuff fizzy pop"

However, she does have a positive relationship with her teacher who gives positive feedback

"I find my school and teachers supportive, specially this one teacher that I've told her about this whole project and I told them that I lost a bit of weight and they were really proud of me so that's like boosted my confidence and make me believe in myself so I am making a difference, so I get motivated."

\begin{tabular}{|c|} 
Social support 12 months \\
\hline She thinks the influence of her friends is mixed as
\end{tabular}
they do support her in her runs but they don't exercise with her. They also offer a source of temptation as they often eat sweets.

"In the middle because the friends I have don't do a lot of exercise so they kind of help me but also kind of don't-If I tell them about the race I do they would congratulate me and tell me I done a job but then if I wanted to do exercising and stuff they don't exactly come and join in with me and come and support me."

"They eat lots of sweets and chocolates and stuff"
"In school I think she just chooses what she wants to eat, again as we said, she finds that difficult in school, when she sees her friends eating all the nice treats", She is motivated by attending the program and is eager to please the researchers

"She wants to say that she has done it [her goals] and please you two [research staff] and keep it going, she likes having people who can see what she's done. If you see the effort that she is trying to put in then she is pleased enough with that, as long as you are happy she is happy, she doesn't like disappointing people, she looks into you two, that's her goal, every time we have a meeting it's like she wants to make sure that she tells you she's done that and she's done this."

\section{Social support 12 months}

Her family are a constant support to her and model healthy behaviours

"She has overcome the $£ 1$ bar because she has always bought that but she's gone a lot smaller than that. But she slips and that's when she is with her friend.

"She doesn't have a group of friends as such, she only has one or two friends and none of them are exercise driven." 


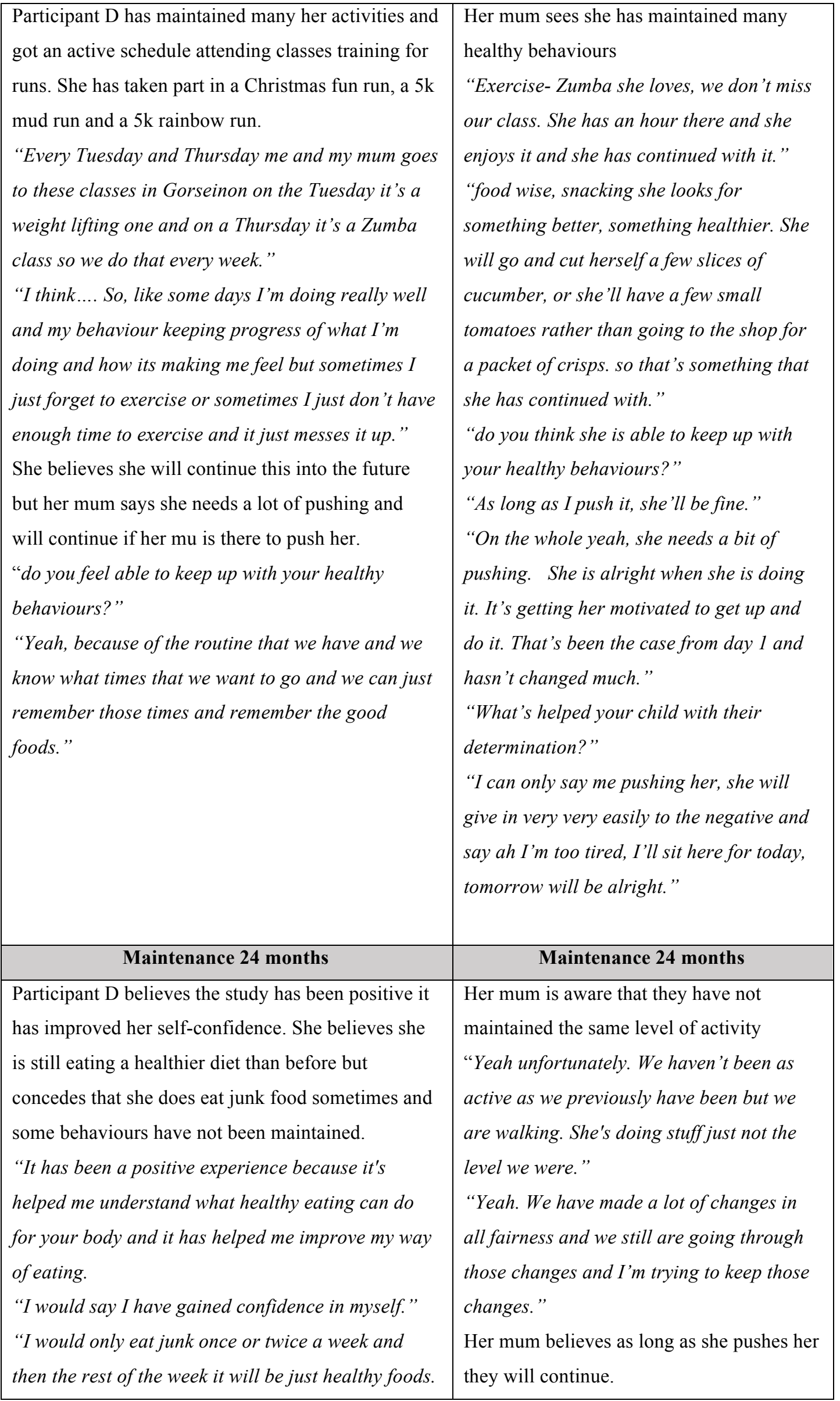




"What about Zumba is that still going?"
"I haven't been for a while."
When asked if she will continue with her healthy
behaviours now that the study is over she is not sure.
"Will your behaviours or your attitude towards
these behaviours change now that the study is
over?"
"I hope not."
"You hope not or you know that you won't or do you
think you might?"
"99\% I won't but $1 \%$ of me is thinking I might."
Visualization and self-talk
Participant D visualizes the changes to her body as a
motivation to exercise and talks to herself in her
head to get through the tough times.
"I visualize me like every month or every week from
the beginning I visualize myself changing every time
I'm exercising and the distance from before and
now because of the differences I see it makes me
want to continue and want to carry on
on the rainbow run I got really exhausted and I got

Relapse 12 months

Participant D describes times when she is not able keep up here healthy behaviours and attributes this to her low mood or family events.

"Like when I am in a bad mood or something I sometimes forget about the project and I have a biscuit or chocolate bar and I have them more than once so that kind of puts me back a step or two"

Going to the shop and buying chocolate bars is still here problem behaviour

"Yeah, but sometimes I do go up there when I have 50 p or something and buy a small bar",
"As long as I keep pushing it, she will be fine. She might lapse. But she is definitely more aware of things that she wasn't aware of before. Sugar content and stuff like that. She is definitely more aware so I think she will keep up."

\section{Relapse 12 months}

"Has your child experienced relapse?"

Yes, we've had a tough couple of months as I told you. We've maintained our two classes. Two classes we do are power fit and Zumba. We have kept them going but we have slackened off a bit but we're building back and we are getting back into the routine that we had and she is happier because she's noticed herself, that she has put on a little bit of weight again or she thinks she has but she's grown as well which is a bit hard to tell, but she notices as well, she says we've gotta get back on what we were doing and she wants to do it 


\begin{tabular}{|c|c|}
\hline & so that always helps." \\
\hline Relapse 24 months (Coping self-efficacy) & Relapse 24 months (Coping self-efficacy) \\
\hline $\begin{array}{l}\text { "Have you experienced any relapses over the past } \\
\text { few months?" } \\
\text { I'm still struggling on the path where my friends are } \\
\text { eating junk and stuff in school. I have a bit of a } \\
\text { relapse in a eating a bit of junk. But recently over } \\
\text { the past few weeks haven't really ate junk. I have } \\
\text { gotten better. } \\
\text { "How did you recover from the relapse" } \\
\text { My mom started giving me packed lunches with } \\
\text { salads wraps and apples and water. } \\
\text { "How are you affected by a relapse?" } \\
\text { It kind of makes me feel down after I have had the } \\
\text { relapse because I don't know that the weight I have } \\
\text { out back on I'll have to start exercising even more } \\
\text { to get off and then continue the normal routine so I } \\
\text { can continue. }\end{array}$ & $\begin{array}{l}\text { "Has she experienced any relapses in the } \\
\text { past months or year?" } \\
\text { "Yeah unfortunately. We haven't been as } \\
\text { active as we previously have been but we } \\
\text { are walking. She's doing stuff just not the } \\
\text { level we were." } \\
\text { "What would you say would be the causes } \\
\text { when he has one of these relapse or times } \\
\text { when he's less committed?" } \\
\text { "My Knee. And she finds it boring } \\
\text { exercising on her own that's why I do it. } \\
\text { But my knee has just stopped everything } \\
\text { unfortunately." } \\
\text { "How did she recover from the relapse?" } \\
\text { "It's just the willingness to try. She wants } \\
\text { to achieve what she wants to achieve. She } \\
\text { has seen previously how good she has felt, } \\
\text { how good the changes have been and she } \\
\text { wants that again. So it's that drive now. } \\
\text { The last couple months haven't been good } \\
\text { but she wants to get to that point again. She } \\
\text { is definitely enthusiastic for it which is } \\
\text { always half the battle." }\end{array}$ \\
\hline
\end{tabular}

